Silicon-Containing Nanoparticles: From Functional Materials to Real-Life Applications 


\section{Silicon-Containing Nanoparticles: \\ From Functional Materials to Real-Life Applications}

Sida Yin 
Graduation Committee:

$\begin{array}{lll}\text { Chairman: } & \text { Prof. dr. J.L. Herek } & \text { University of Twente } \\ \text { Promotor: } & \text { Prof. dr. G.J. Vancso } & \text { University of Twente } \\ \text { Assistant-promotor: } & \text { Dr. ir. J. Duvigneau } & \text { University of Twente } \\ \text { Members: } & \text { Prof. dr. ir. L. Lefferts } & \text { University of Twente } \\ & \text { Prof. dr. A. Blume } & \text { University of Twente } \\ & \text { Prof. dr. Ing. N. Benson } & \text { Universität Duisburg/Essen } \\ & \text { Dr. O.J. Nguon } & \text { University of Twente } \\ & \text { Prof. ir. M. Hedenqvist } & \begin{array}{l}\text { KTH Royal Institute of } \\ \text { Technology }\end{array}\end{array}$

The research described in this Thesis was carried out in the Materials Science and Technology of Polymers (MTP) group (later Sustainable Polymer Chemistry group), MESA+ Institute for Nanotechnology, Faculty of Science and Technology, University of Twente, the Netherlands.

This research was financially supported by the MESA+ Institute for Nanotechnology, University of Twente, and by the Interreg V project Druckbare RFID - Tags für Massenmärkte (DruIDe).

Cover design: Sida Yin

Printed by: PROEFSCHRIFTMAKEN

ISBN: 978-90-365-5309-4

DOI: $10.3990 / 1.9789036553094$

Copyright (C) 2021 Sida Yin, The Netherlands. All rights reserved. No parts of this Thesis may be reproduced, stored in a retrieval system or transmitted in any form or by any means without permission of the author. Alle rechten voorbehouden. Niets uit deze uitgave mag worden vermenigvuldigd, in enige vorm of op enige wijze, zonder voorafgaande schriftelijke toestemming van de auteur. 


\title{
DISSERTATION
}

\author{
to obtain \\ the degree of doctor at the Universiteit Twente, \\ on the authority of the rector magnificus, \\ prof. dr. ir. A. Veldkamp, \\ on account of the decision of the Doctorate Board \\ to be publicly defended \\ on Wednesday 15 December 2021 at 12.45 hours
} by

Sida Yin

born on the $4^{\text {th }}$ of August, 1990 in Hebei, China 
This dissertation has been approved by:

Promotor:

Prof. dr. G.J. Vancso

University of Twente

Assistant-promotor:

Dr. ir. J. Duvigneau

University of Twente 


\section{Contents}

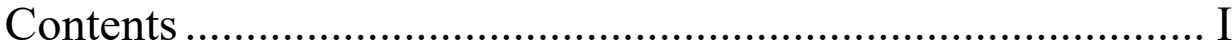

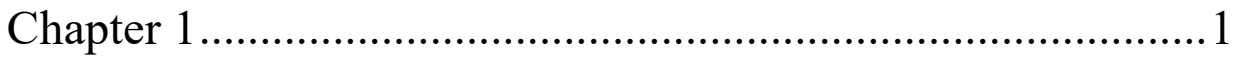

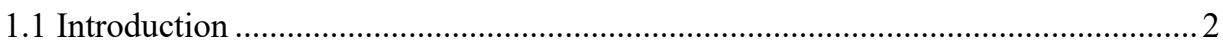

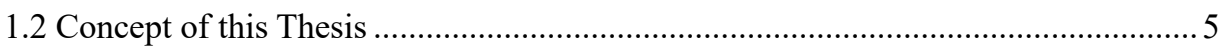

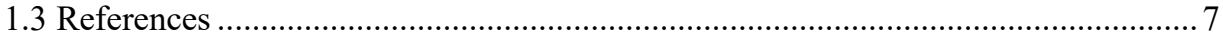

Chapter 2 ................................................................. 11

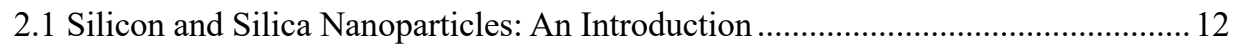

2.2 Silicon-Containing Nanoparticle Production ...................................................... 14

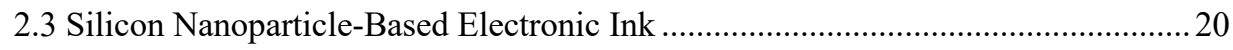

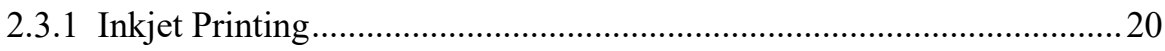

2.3.2 Surface Oxidation of Silicon Nanoparticles ...................................... 22

2.3.3 Surface Modification of Silicon Nanoparticles to Fabricate Stable

Colloidal Inks ............................................................................. 25

2.3.4 Inkjet Printing of Silicon Nanoparticle Inks ................................... 30

2.4 Silicon Nanoparticles as the Next Generation "Magic Bullets" in Biomedical

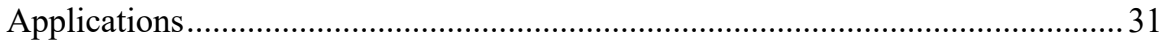

2.4.1 Silicon Nanoparticles as Drug Delivery Carriers ................................. 32

2.4.2 Photoluminescent Silicon Nanoclusters and Bioimaging ..................... 34

2.4.3 Silicon Nanoparticle and Magnetic Resonance Imaging ...................... 36

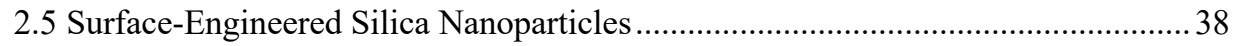

2.5.1 Surface Roughness Engineering of Silica Nanoparticles ..................... 38

2.5.2 Foam Cell Nucleation by Surface Topology Engineered Silica

Nanoparticles .......................................................................... 41

2.5.3 Polymer Nanocomposite Foams .................................................... 43

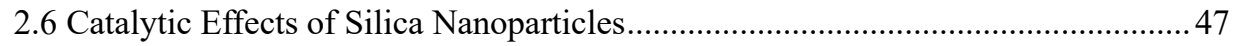

2.6.1 Catalytic Activities of Silica Nanoparticles ..................................... 47 


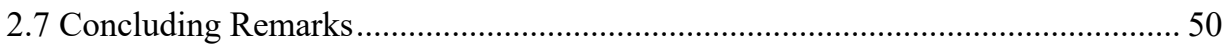

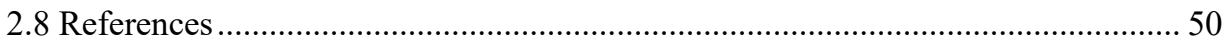

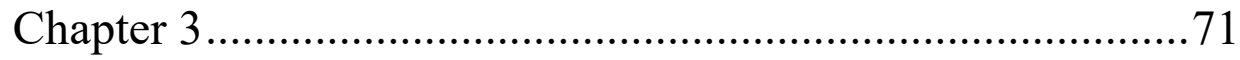

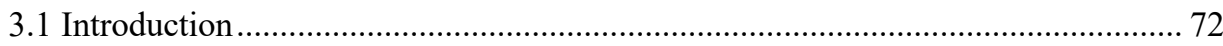

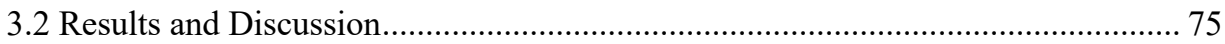

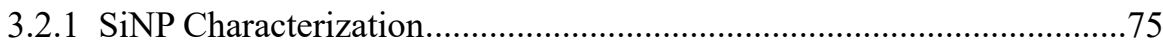

3.2.2 AC to Study SiNP Sedimentation Behavior in Various Solvents ...........79

3.2.3 Batch Sedimentation Evaluation of SiNP Dispersions .........................82

3.2.4 Ethylene Glycol and Diethylene Glycol Stability in the Presence of

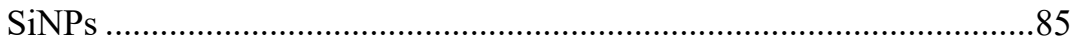

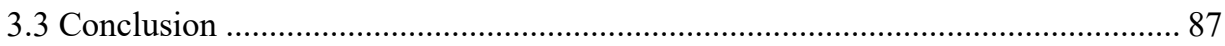

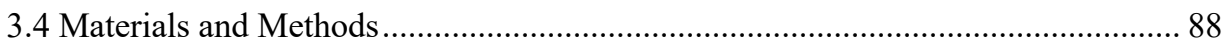

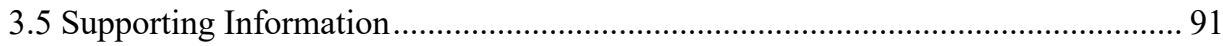

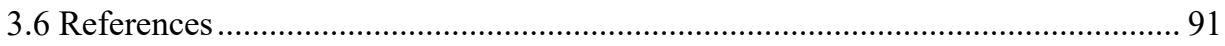

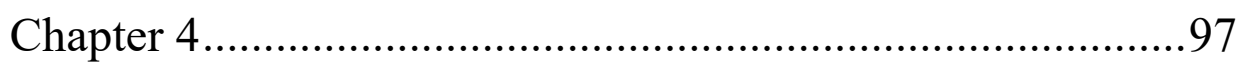

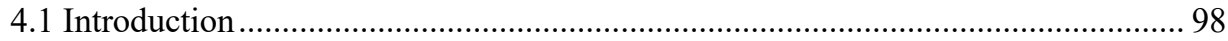

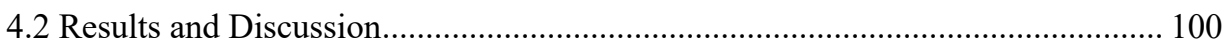

4.2.1 Printing Test of SiNP-Based Inks ...............................................100

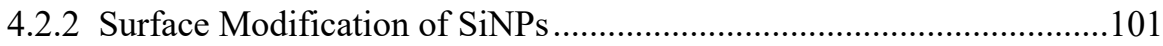

4.2.3 Characterization of Polymer-Nanoparticle Hybrids ...........................102

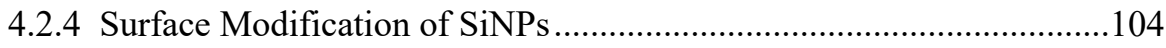

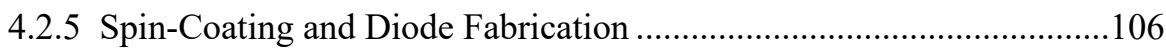

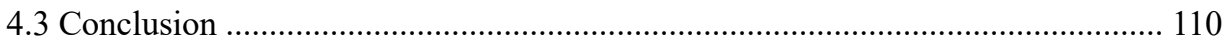

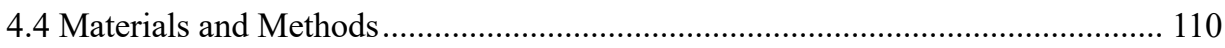

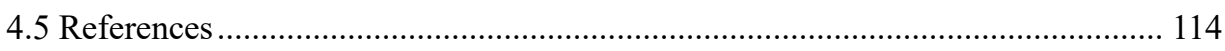

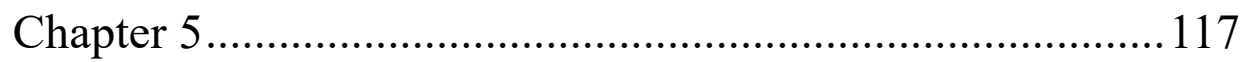

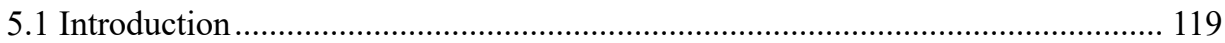




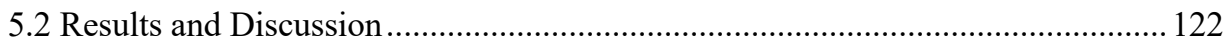

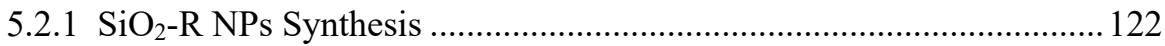

5.2.2 Nanocomposite Foaming and Multinucleation ……………………..... 126

5.2.3 Multiple Foam Cells Nucleation Mechanism......................................... 130

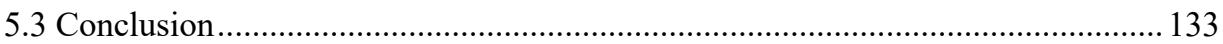

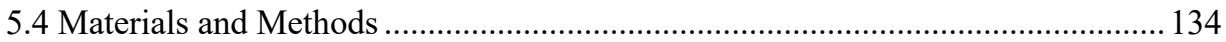

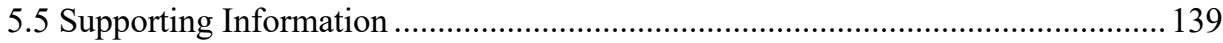

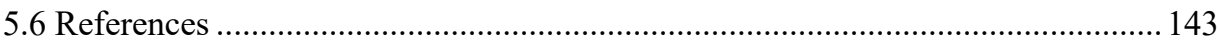

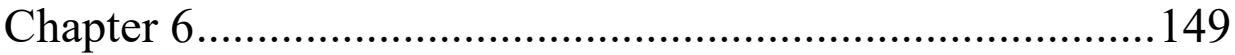

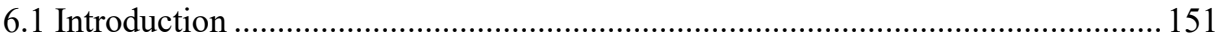

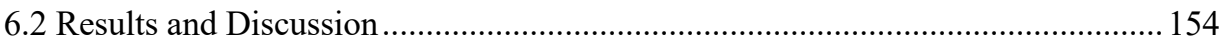

6.2.1 $\mathrm{SiO}_{2}$ Synthesis and Characterization..................................................... 154

6.2.2 $\mathrm{PE} / \mathrm{SiO}_{2}$ Composites and Fluorescence Microscopy ……....................... 154

6.2.3 The Origin of the Observed Fluorescent: CQD Isolation and

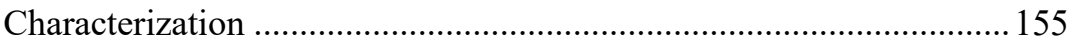

6.2.4 Role of $\mathrm{SiO}_{2}$ NPs in CQD Formation in Heated PE/SiO 2 Solutions.... 157

6.2.5 Effect of $\mathrm{SiO}_{2} \mathrm{NPs}$ Concentration of Fluorescent Intensity of $\mathrm{PE} / \mathrm{SiO}_{2}$

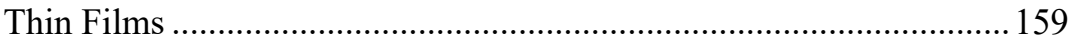

6.2.6 Fluorescent Spectra of $\mathrm{PE} / \mathrm{SiO}_{2}$ Thin Films and the Influence of the Reaction Temperature.................................................................... 160

6.2.7 Solution Blending CQDs and $\mathrm{PE} / \mathrm{SiO}_{2}$ Composites with Pure PE....... 162

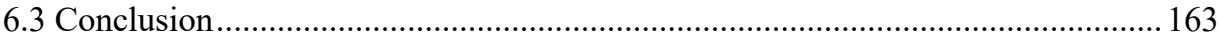

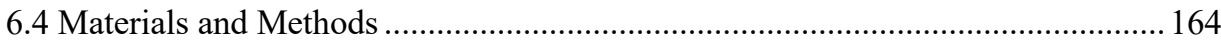

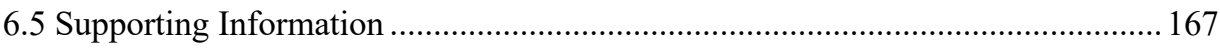

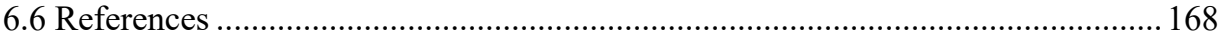

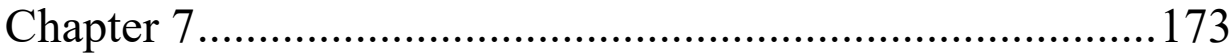

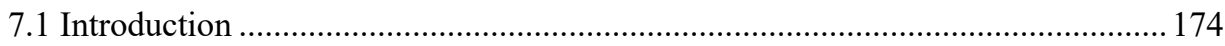


7.2 Long-Term Stability and Printability of SiNP-Based Inks 174

7.3 Strategies for Polymer Immobilization on SiNP Surfaces 176

7.4 SiNPs as Dual Probe for Magnetic Resonance and Fluorescent Imaging. 177

7.5 References 180

Summary 183

Samenvatting 187

Acknowledgements 191

List of Publications. 193

Curriculum Vitae. 195 
Chapter 1

General Introduction 


\subsection{Introduction}

Nanoparticles (NPs) are defined as particles with at least one dimension smaller than $100 \mathrm{~nm}$. Although NPs in nanotechnology have been introduced formally only several decades ago, humankind has been using NPs for thousands of years. ${ }^{1,2}$ For example, the famous Lycurgus Cup from $4^{\text {th }}$ century Rome uses metal NPs to produce dichroism. ${ }^{1}$ The glass cup appears to be green in reflected light. However, when light passes through, the cup seems to be red and purple. The color change is attributed to the dispersion of gold and silver NPs in the glass matrix that scatter the incoming light. ${ }^{1}$

Compared to their bulk form, NPs possess an extremely high surface-to-volume ratio. Therefore, a high percentage of atoms/molecules are exposed at the NPs' surface. As a result, NPs often have unique physicochemical properties, e.g., magnetism, thermal/electrical conductivities, mechanical properties, and antimicrobial activity. ${ }^{3,4}$ Interestingly, since the percentage of surface atoms/molecules is strongly correlated to the particle size, NPs usually have size-tunable physicochemical properties (see Figure $1.1) . .^{3,5}$

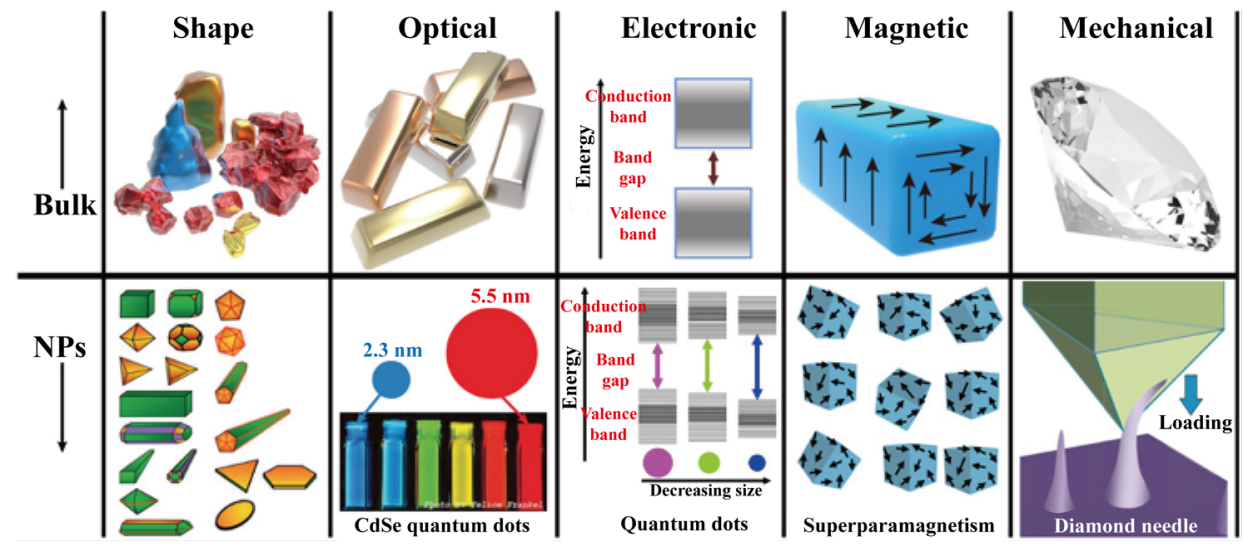

Figure 1.1 Comparison of characteristics of bulk materials and corresponding NPs. ${ }^{6}$

NPs are either made by breaking down bulk materials (top-down) or controlled assembly (bottom-up). Both natural phenomena and human activities can produce NPs. ${ }^{7}$ For example, fire results in the formation of carbon NPs. ${ }^{7}$ Living organisms like bacteria and fungi are also masters in nanotechnology in NP fabrication. For instance, bacteria produce selenium NPs by reducing selenite in dairy products. ${ }^{8}$ Nowadays, a wide range of materials and methods are used to make NPs.

Silicon is a particularly interesting material for nanotechnology. Si is the second most 
abundant element, and when it is combined with the most abundant element on Earth, oxygen, silicon oxides are formed. Both silicon and silicon oxides have been used to form silicon-containing (Si-containing) NPs with exciting properties (see Figure 1.2). Sicontaining NPs have attracted considerable attention from scientists and engineers across multiple disciplines. ${ }^{9,10}$ These Si-containing NPs represent a highly active research field and they are used in several industrial sectors, e.g., electronics, cosmetics, and healthcare. ${ }^{9,11-14}$ In this Thesis, we focuse on designing and utilizing non-porous silicon NPs (SiNPs) and non-porous Silica $\left(\mathrm{SiO}_{2}\right)$ NPs in emerging application areas, e.g., printable electronics, nanocellular foaming, and chemical applications reaction enablers.

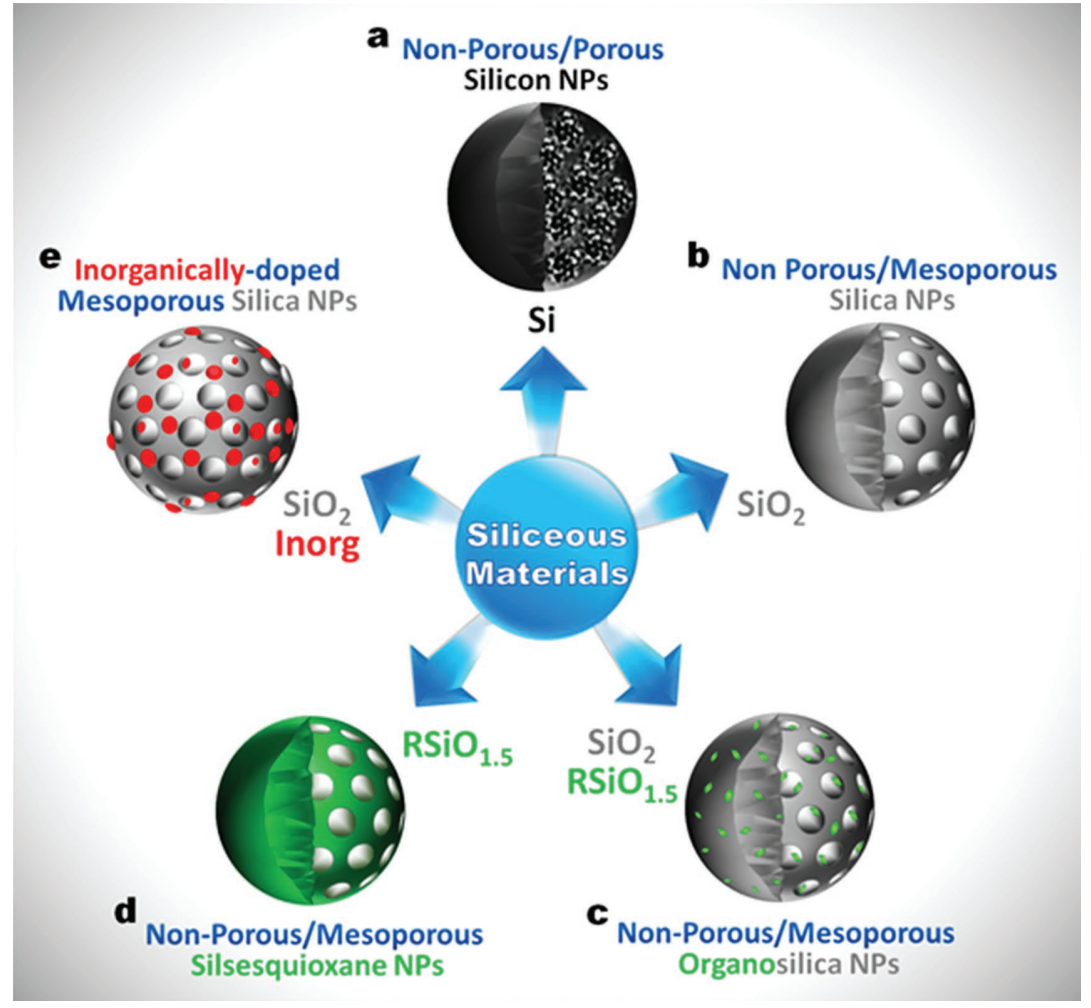

Figure 1.2 Representation of various Si-containing NPs. ${ }^{15}$

SiNPs comprise one of the most abundantly used classes of Si-containing NPs. SiNPs are well-known for their photoluminescent properties and size-dependent bandgap. ${ }^{16}$ SiNPs have been thoroughly studied in various application areas, e.g., light-emitting diodes (LEDs) ${ }^{17}$, drug delivery ${ }^{18}$, sensors ${ }^{19,20}$ and bio-imaging ${ }^{21-24}$. Printing of such SiNPs is considered one of the most promising techniques for the large-scale fabrication of electronic devices. ${ }^{25}$ However, SiNPs have not yet been widely utilized as electronic 
inks for printing like other metal NPs, even though bulk silicon is considered the cornerstone of modern electronics. ${ }^{26}$ To the best of our knowledge, only a few recent reports describe the use of SiNPs as components in inks to print electronic devices. ${ }^{26-29}$ This is attributed to the highly reactive surface of SiNPs and the inefficient approaches to prepare stable SiNP suspensions in large quantities. ${ }^{30}$ Moreover, the influence of wellestablished surface derivatization strategies for stabilizing these dispersions, e.g. by utilizing polymer grafts, on the printability and electrical performance of SiNP-based devices is not yet thoroughly understood. This Thesis contributes to gaining a detailed insight into these fundamentally interesting aspects of NP science and engineering. Firstly, we prepared SiNP suspensions with different organic solvents to evaluate the accuracy of preexisting methods to predict the long-term colloidal stability of non-ideal SiNPs. Secondly, we investigated the influence of polymer grafts on the stability and printability of SiNP hybrid-based inks for printing electronic devices, including assessing the functionality of the printed devices.

$\mathrm{SiO}_{2} \mathrm{NPs}$ form another exciting class of silicon-based NPs that received a considerable amount of attention from the nanotechnology community. $\mathrm{SiO}_{2} \mathrm{NPs}$ can be formed in well developed synthetic approaches, and their properties can then be altered by a wide range of modification. ${ }^{31,32}$ They have been widely utilized in drug delivery, nano-structuring, and bioimaging applications. ${ }^{33,34}$ The Materials Science and Technology of Polymers (MTP) group has recently presented and discussed the utilization of hybrid $\mathrm{SiO}_{2}$ NPs as high-efficiency heterogeneous foam cell nucleation agents in $\mathrm{CO}_{2}$-based batch foaming of polymer nanocomposites. ${ }^{35,36}$ To date, the highest nucleation efficiency, i.e., the number of cells obtained per $\mathrm{SiO}_{2} \mathrm{NP}$ added, reported by the MTP group was $\sim 0.5$ for poly(dimethylsiloxane) (PDMS) grafted spherical $80 \mathrm{~nm}$ $\mathrm{SiO}_{2} \mathrm{NPs}^{36}$ In this Thesis, we show that the utilization of surface roughness engineered PDMS grafted $\mathrm{SiO}_{2}$ NPs can further enhance the nucleation efficiency to values exceeding unity. This opens the possibility of applying new foaming strategies at relatively low saturation pressure (below 60 bar) while maintaining reasonable high cell densities and small cell sizes.

In addition, chemically inert mesoporous silica nanoparticles (MSNs) have been widely implemented as catalyst support for metal-based catalysts. ${ }^{37,38}$ Moreover, it has been reported in literature that the pores of MSNs' can act like nanometric reactors and encapsulate free radicals during polymer degradation. ${ }^{39}$ The encapsulation of radicals extend their lifetime, which was demonstrated to accelerate polymer degradation. However, the synthetic approaches to prepare MSNs typically involve complex chemical reactions, extended washing steps, and long-time calcination, ${ }^{40,41}$ hampering their low- 
cost industrial utilization. Compared to $\mathrm{MSNs}$, Stöber $\mathrm{SiO}_{2}$ particles are typically obtained by mild reaction conditions. ${ }^{42}$ Moreover, the well-established Stöber process is easily and cost-effectively scaled to industrial levels. We will demonstrate that carbon quantum dots can be obtained by the thermal degradation of polyethylene at mild reaction conditions in the presence of agglomerated $\mathrm{SiO}_{2}$ NPs. We speculate that the $\mathrm{SiO}_{2}$ aggregate pores prolong the lifetime of created $\mathrm{PE}$ radicals and, therefore, accelerate $\mathrm{PE}$ degradation to yield CQDs at modest reaction conditions.

Overall we believe that the results obtain in this Thesis contribute to a better understanding of Si-containing NPs in the presented emerging application areas.

\subsection{Concept of this Thesis}

Si-containing NPs with different morphologies and surface functionalities have been thoroughly studied and widely implemented in multiple application fields during the last few decades. Among the broad diversity of reported Si-containing NPs, $\mathrm{SiNPs}_{\text {and }} \mathrm{SiO}_{2}$ NPs are two promising examples with unique capabilities for several interesting applications. However, a challenge in utilizing the application potential of Si-containing NPs ( $\mathrm{SiNPs}$ and $\mathrm{SiO}_{2} \mathrm{NPs}$ ) is related to the relative infancy of bridging advanced laboratory research and real technological use. With the swift progress in the enabling tools and methods in nanotechnology, new windows have been opened to tackle still open problems (or notoriously difficult challenges) in Si-containing NP research. In this Thesis, we address some of these challenges to ensure continuous progress in the field.

The literature covering general aspects and synthetic strategies of Si-containing NPs is reviewed in Chapter 2. Moreover, recent developments of Si-containing NPs in the selected emerging application areas presented in the subsequent chapters are briefly discussed. This Chapter provides an outline to understand better the opportunities and challenges for integrating Si-containing NPs into real-life applications.

In Chapter 3, we investigate the long-term colloidal stability of SiNPs with a complex geometry and broad size distribution. Theoretical (Stokes' law) and experimental (analytical centrifugation and batch sedimentation testing) approaches are used to predict and measure the colloidal stability of SiNPs in different alcohols and glycols. The insights obtained serve as a first step towards formulating long-term stable complex SiNPs-based inks for printable electronic devices (e.g., RFID tags). Furthermore, the results highlighted the need for surface modification of SiNPs to enhance their long-term colloidal stability in glycols. 
Based on the results obtained in Chapter 3, core-shell SiNP/polymer hybrids with poly (methyl methacrylate) and poly (ethylene glycol) methacrylate shells are synthesized through surface-initiated atom transfer radical polymerization (SI-ATRP) to enhance stability and printability of SiNP-based inks for printable electronic devices. The grafted polymer shells help reduce nozzle clogging during printing and prevent potential interactions between particles and solvent during storage. The I-V (current-voltage) characteristics of printed, electronic devices from SiNP/polymer hybrids are evaluated. Furthermore, the I-V characteristics of the printed devices point towards the need to develop the functional SiNP-based electronic inks further.

In Chapter 5, surface roughness engineered $\mathrm{SiO}_{2} \mathrm{NPs}\left(\mathrm{SiO}_{2}-\mathrm{R}\right.$ NPs) with PDMS shells are synthesized and exploited as heterogeneous nucleation agents for $\mathrm{CO}_{2}$ batch foaming of PMMA nanocellular foams. By combining the reduced free energy for foam cell nucleation inside nanocavities on the particle surface and the high $\mathrm{CO}_{2}$ absorption of the PDMS shell, multiple foam cells are nucleated from a single particle. This extremely efficient nucleation behavior renders $\mathrm{SiO}_{2}-\mathrm{R}$ NPs highly promising nucleation agents for polymer nanocellular foaming.

In Chapter 6, carbon quantum dots (CQDs) fluorescent markers containing polyethylene (PE) are synthesized at mild reaction conditions using $\mathrm{SiO}_{2} \mathrm{NP}$ aggregates as enablers. By taking advantage of the nano-pores residing on the surface of the aggregates, the PE chains that enter the pores can degrade and form CQDs at low temperatures $\left(90-110^{\circ} \mathrm{C}\right)$. Moreover, we demonstrate that PE/CQD composites and $\mathrm{PE}$ samples loaded with extracted CQDs could be visually separated from pure unmarked PE upon irradiation with $367 \mathrm{~nm}$ light. Thus this Chapter provides a facile solution to synthesize fluorescent markers that are readily used as labeling ingredients for component identification and plastic waste recycling.

Finally, in Chapter 7, we provide an outlook for future directions of SiNPs in printable electronics and bioimaging. For SiNP-based inks, we present and discuss selfimmolating polymers and catalyst-free hydrosilylation as the next essential step to enable the large-scale production of hybrid SiNP-based inks with enhanced colloidal stability and printability, with a minimum impact on the performance of printed, electronic devices. In addition, some preliminary results on utilizing multi-crystalline SiNPs as a dual probe for magnetic resonance imaging and fluorescent imaging are presented. 


\subsection{References}

(1) Freestone, I.; Meeks, N.; Sax, M.; Higgitt, C. The Lycurgus Cup - A Roman Nanotechnology. Gold Bulletin 2008, 40 (4), 270-277.

(2) Landauer, R. (2018) Information Is Inevitably Physical. In A. J. G. Hey (Ed.). Feynman and Computation (pp 77-92). CRC Press.

(3) Khan, I.; Saeed, K.; Khan, I. Nanoparticles: Properties, Applications and Toxicities. Arabian Journal of Chemistry 2019, 12 (7), 908-931.

(4) Baig, N.; Kammakakam, I.; Falath, W.; Kammakakam, I. Nanomaterials: A Review of Synthesis Methods, Properties, Recent Progress, and Challenges. Materials Advances 2021, 2 (6), 1821-1871.

(5) Jeevanandam, J.; Barhoum, A.; Chan, Y. S.; Dufresne, A.; Danquah, M. K. Review on Nanoparticles and Nanostructured Materials: History, Sources, Toxicity and Regulations. Beilstein Journal of Nanotechnology 2018, 9 (1), 1050 1074.

(6) Huang, C.; Chen, X.; Xue, Z.; Wang, T. Effect of Structure: A New Insight into Nanoparticle Assemblies from Inanimate to Animate. Science Advances 2020, 6 (20), 10.1126/sciadv.aba1321.

(7) Griffin, S.; Masood, M. I.; Nasim, M. J.; Sarfraz, M.; Ebokaiwe, A. P.; Schäfer, K. H.; Keck, C. M.; Jacob, C. Natural Nanoparticles: A Particular Matter Inspired by Nature. Antioxidants 2018, 7 (1), 3 .

(8) Wright, M. H.; Farooqui, S. M.; White, A. R.; Greene, A. C. Production of Manganese Oxide Nanoparticles by Shewanella Species. Applied and Environmental Microbiology 2016, 82 (17), 5402-5409.

(9) Jeelani, P. G.; Mulay, P.; Venkat, R.; Ramalingam, C. Multifaceted Application of Silica Nanoparticles. A Review. Silicon 2020, 12 (6), 1337-1354.

(10) Chang, H.; Sun, S. Q. Silicon Nanoparticles: Preparation, Properties, and Applications. Chinese Physics B 2014, 23 (8), 1-14.

(11) Nayfeh, M. H.; Mitas, L. (2008) Silicon Nanoparticles. New Photonic and Electronic Material at the Transition Between Solid and Molecule. In V. Kumar (Ed.). Nanosilicon (pp 1-78). Elsevier.

(12) O'Farrell, N.; Houlton, A.; Horrocks, B. R. Silicon Nanoparticles: Applications in Cell Biology and Medicine. International Journal of Nanomedicine 2006, 1 (4), 451-472.

(13) Downing, M. A.; Jain, P. K. Mesoporous Silica Nanoparticles: Synthesis, Properties, and Biomedical Applications. Nanoparticles for Biomedical Applications: Fundamental Concepts, Biological Interactions and Clinical Applications 2019, 42 (10), 267-281.

(14) Capeletti, L. B.; Loiola, L. M. D.; Picco, A. S.; da Silva Liberato, M.; Cardoso, M. B. Silica Nanoparticle Applications in the Biomedical Field. Smart Nanoparticles for Biomedicine 2018, 17 (19-20), 115-129.

(15) Croissant, J. G.; Fatieiev, Y.; Khashab, N. M. Degradability and Clearance of Silicon, Organosilica, Silsesquioxane, Silica Mixed Oxide, and Mesoporous Silica Nanoparticles. Advanced Materials 2017, 29 (9), 10.1002/adma.201604634.

(16) Meier, C.; Gondorf, A.; Lüttjohann, S.; Lorke, A.; Wiggers, H. Silicon Nanoparticles: Absorption, Emission, and the Nature of the Electronic Bandgap. 
Journal of Applied Physics 2007, 101 (10), 103112.

(17) Maier-Flaig, F.; Rinck, J.; Stephan, M.; Bocksrocker, T.; Bruns, M.; Kübel, C.; Powell, A. K.; Ozin, G. A.; Lemmer, U. Multicolor Silicon Light-Emitting Diodes (SiLEDs). Nano Letters 2013, 13 (2), 475-480.

(18) Haidary, S. M.; Córcoles, E. P.; Ali, N. K. Nanoporous Silicon as Drug Delivery Systems for Cancer Therapies. Journal of Nanomaterials 2012, 2012, 830503.

(19) Jalkanen, T.; Mäkilä, E.; Määttänen, A.; Tuura, J.; Kaasalainen, M.; Lehto, V. P.; Ihalainen, P.; Peltonen, J.; Salonen, J. Porous Silicon Micro- and Nanoparticles for Printed Humidity Sensors. Applied Physics Letters 2012, 101 (26), 263110.

(20) Chu, B.; Wang, H.; Song, B.; Peng, F.; Su, Y.; He, Y. Fluorescent and Photostable Silicon Nanoparticles Sensors for Real-Time and Long-Term Intracellular PH Measurement in Live Cells. Analytical Chemistry 2016, 88 (18), 9235-9242.

(21) Kwiatkowski, G.; Jähnig, F.; Steinhauser, J.; Wespi, P.; Ernst, M.; Kozerke, S. Nanometer Size Silicon Particles for Hyperpolarized MRI. Scientific Reports 2017, 7 (1), 7946.

(22) Whiting, N.; Hu, J.; Zacharias, N. M.; Lokesh, G. L. R.; Volk, D. E.; Menter, D. G.; Rupaimoole, R.; Previs, R.; Sood, A. K.; Bhattacharya, P. Developing Hyperpolarized Silicon Particles for in Vivo MRI Targeting of Ovarian Cancer. Journal of Medical Imaging 2016, 3 (3), 036001.

(23) Ji, X.; Peng, F.; Zhong, Y.; Su, Y.; Jiang, X.; Song, C.; Yang, L.; Chu, B.; Lee, S. T.; He, Y. Highly Fluorescent, Photostable, and Ultrasmall Silicon Drug Nanocarriers for Long-Term Tumor Cell Tracking and in-Vivo Cancer Therapy. Advanced Materials 2015, 27 (6), 1029-1034.

(24) Whiting, N.; Hu, J.; Shah, J. v.; Cassidy, M. C.; Cressman, E.; Millward, N. Z.; Menter, D. G.; Marcus, C. M.; Bhattacharya, P. K. Real-Time MRI-Guided Catheter Tracking Using Hyperpolarized Silicon Particles. Scientific Reports 2015, 5, 12842.

(25) Zeng, M.; Zhang, Y. Colloidal Nanoparticle Inks for Printing Functional Devices: Emerging Trends and Future Prospects. Journal of Materials Chemistry A 2019, 7 (41), 23301-23336.

(26) Gupta, A.; Khalil, A. S. G.; Offer, M.; Geller, M.; Winterer, M.; Lorke, A.; Wiggers, H. Synthesis and Ink-Jet Printing of Highly Luminescing Silicon Nanoparticles for Printable Electronics. Journal of Nanoscience and Nanotechnology 2011, 11 (6), 5028-5033.

(27) Lawes, S.; Sun, Q.; Lushington, A.; Xiao, B.; Liu, Y.; Sun, X. Inkjet-Printed Silicon as High Performance Anodes for Li-Ion Batteries. Nano Energy 2017, 36, 313-321.

(28) Drahi, E.; Blayac, S.; Borbely, A.; Benaben, P. Impact of Ink Synthesis on Processing of Inkjet-Printed Silicon Nanoparticle Thin Films: A Comparison of Rapid Thermal Annealing and Photonic Sintering. Thin Solid Films 2015, 574, 169-176.

(29) Drahi, E.; Gupta, A.; Blayac, S.; Saunier, S.; Benaben, P. Characterization of Sintered Inkjet-Printed Silicon Nanoparticle Thin Films for Thermoelectric Devices. Physica Status Solidi (A) Applications and Materials Science 2014, 211 (6), 1301-1307.

(30) Sailor, M. J. (2017). Chemical Reactivity and Surface Chemistry of Porous 
Silicon. In L. Canham (Ed.). Handbook of Porous Silicon (pp 1-20). Springer International Publishing, Cham.

(31) Badley, R. D.; Ford, W. T.; McEnroe, F. J.; Assink, R. A. Surface Modification of Colloidal Silica. Langmuir 1990, 6 (4), 792-801.

(32) Ibrahim, I. a. M.; Zikry, a. a. F.; Sharaf, M. a. Preparation of Spherical Silica Nanoparticles: Stober Silica. Journal of American Science 2010, 6 (11), 985-989.

(33) Barbé, C.; Bartlett, J.; Kong, L.; Finnie, K.; Lin, H. Q.; Larkin, M.; Calleja, S.; Bush, A.; Calleja, G. Silica Particles: A Novel Drug-Delivery System. Advanced Materials 2004, 16 (21), 1959-1966.

(34) Korzeniowska, B.; Nooney, R.; Wencel, D.; McDonagh, C. Silica Nanoparticles for Cell Imaging and Intracellular Sensing. Nanotechnology 2013, 24 (44), 442002.

(35) Liu, S.; Yin, S.; Duvigneau, J.; Vancso, G. J. Bubble Seeding Nanocavities: Multiple Polymer Foam Cell Nucleation by Polydimethylsiloxane-Grafted Designer Silica Nanoparticles. ACS Nano 2020, 14 (2), 1623-1634.

(36) Liu, S.; Zoetebier, B.; Hulsman, L.; Zhang, Y.; Duvigneau, J.; Vancso, G. J. Nanocellular Polymer Foams Nucleated by Core-Shell Nanoparticles. Polymer 2016, 104, 22-30.

(37) Shinde, P. S.; Suryawanshi, P. S.; Patil, K. K.; Belekar, V. M.; Sankpal, S. A.; Delekar, S. D.; Jadhav, S. A. A Brief Overview of Recent Progress in Porous Silica as Catalyst Supports. Journal of Composites Science 2021, 5 (3), 75.

(38) Yu Lai, Dr. C. Mesoporous Silica Nanomaterials Applications in Catalysis. Journal of Thermodynamics \& Catalysis 2014, 05 (01), 10-12.

(39) Sakata, Y.; Azhar Uddin, M.; Muto, A.; Kanada, Y.; Koizumi, K.; Murata, K. Catalytic Degradation of Polyethylene into Fuel Oil over Mesoporous Silica (KFS-16) Catalyst. Journal of Analytical and Applied Pyrolysis 1997, 43 (1), 1525.

(40) Mehmood, A.; Ghafar, H.; Yaqoob, S.; Gohar, U. F.; Ahmad, B. Mesoporous Silica Nanoparticles: A Review. Journal of Developing Drugs 2017, 06 (02), 10.4172/2329-6631.1000174.

(41) Narayan, R.; Nayak, U. Y.; Raichur, A. M.; Garg, S. Mesoporous Silica Nanoparticles: A Comprehensive Review on Synthesis and Recent Advances. Pharmaceutics 2018, 10 (3), 118.

(42) Stöber, W.; Fink, A.; Bohn, E. Controlled Growth of Monodisperse Silica Spheres in the Micron Size Range. Journal of Colloid And Interface Science 1968, 26 (1), 62-69. 



\section{Chapter 2}

\section{Emerging Applications of Silicon-Containing Nanoparticles}

Silicon-containing nanoparticles are considered a promising class of materials with exciting physical properties and the potential to revolutionize many emerging application areas. This Chapter introduces the fundamental aspects of silicon-containing nanoparticles and their most common production approaches. In addition, we provide a comprehensive overview of the frontiers of silicon-containing nanoparticle research for the growing fields of printable Silicon-containing electronics, theranostics, nanocellular polymer foaming, and catalysis. We include an introduction to readily applicable particle surface modification strategies as well. Finally, the limitations and challenges for the successful commercialization of silicon-containing nanoparticles in the application fields mentioned are also presented and discussed.

The contents of this Chapter will be published as: Yin, S.; Duvigneau, J.; Vancso, G. J. Emerging Applications of Silicon-Containing Nanoparticles. In preparation. 


\subsection{Silicon and Silica Nanoparticles: An Introduction}

Silicon ( $\mathrm{Si}$ ) is the second most abundant element on earth. It is a tetravalent semiconductor that is widely used in numerous fields. ${ }^{1,2}$ When combining Si with the most abundant element on Earth, oxygen, silicon oxides are formed, of which $\mathrm{SiO}_{2}$ is the most naturally occurring. Almost all electronic devices produced today contain Sicontaining components like integrated circuit chips, metal-oxide-Si field-effect transistors, or semiconductor memories. ${ }^{3}$ With the continuous downsizing of siliconcontaining (Si-containing) electronic components, the interest in structure-property relationships of nano-sized Si-containing compounds is swiftly increasing.

Nanoparticles (NPs) are typically defined as microscopically small particles with at least one of their dimensions below $100 \mathrm{~nm} .{ }^{4}$ Compared to their corresponding bulk form, NPs of the same material often possess unique properties that can open new application areas. ${ }^{4}$ Numerous research efforts have been dedicated to the investigation and utilization of $\mathrm{Si}$ - and $\mathrm{SiO}_{2}$-containing nanomaterials. ${ }^{5-8}$

Upon entering the nanoscale realm, Si particles start to reveal new properties - like photoluminescence (PL), antimicrobial properties, and a size-dependent electronic bandgap ${ }^{9,10}$ - that are not observed for bulk silicon. Depending on the preparation method, silicon nanoparticles (SiNPs) are either amorphous or crystalline, with diameters ranging from less than one nanometer to a few hundred nanometers. ${ }^{9}$ Due to the highly active $\mathrm{H}-\mathrm{Si}-\mathrm{Si}-\mathrm{H}$ surface structure, SiNPs tend to oxidize (aging) when stored in ambient conditions, which results in the formation of a surface-exposed inert $\mathrm{SiO}_{2}$ layer. ${ }^{11}$

One of the most intriguing and studied properties of SiNPs is their luminescent ability in the visible spectrum. ${ }^{12}$ In the 1990 s, Canham ${ }^{13}$ discovered visible red PL of electrochemically etched $\mathrm{Si}$ at room temperature. Nearly three decades after the first discovery of PL from SiNPs, the origin of the PL from SiNPs is still under debate. ${ }^{14-16}$ The most popular explanation for the observed PL is that by reducing the size of the bulk crystal to nanocrystals or even nanoclusters, the electrons in the material experience quantum confinement, which increases the bandgap. ${ }^{17}$ In bulk materials, the band gaps are merged to form a conduction band. By decreasing the material's size, the bandgap increases (see Figure 2.1), resulting in discrete energy levels, which eventually leads to size-dependent PL. The PL of SiNPs possesses several unique properties like i) a broad range of absorption and excitation wavelengths, ii) color (emission) tunability, and iii) monochromatic light emission. ${ }^{18}$ 
Numerous studies have been carried out to explore new application areas for PL SiNPs, such as in sensors ${ }^{19,20}$ and bio-imaging ${ }^{21,22}$. For example, Maier-Flaig et al. ${ }^{23}$ reported multicolor Si-containing light-emitting diodes (Si LED) using well-defined, size-separated SiNPs. SiNPs are also promising candidates in the photovoltaic industry. For instance, the integration of ultrathin films of luminescent SiNPs in polycrystalline solar cells was demonstrated by Stupca et al. ${ }^{24}$ By incorporating $1 \mathrm{~nm}$ blue luminescent or $2.85 \mathrm{~nm}$ red luminescent SiNPs, they managed to enhance the power performance by $60-70 \%$ in the UV region and $10 \%$ in the visible region. Similar to the origin of PL, the correlation between the size and PL for SiNPs is still not fully elucidated. However, based on the calculation of the quantum confinement effect, SiNPs need to be smaller than 5 $\mathrm{nm}$ to be photoluminescent. ${ }^{25}$

When looking at silica nanoparticles $\left(\mathrm{SiO}_{2} \mathrm{NPs}\right)$, one learns that they are readily dispersed in aqueous solutions and even to some degree biodegradable. ${ }^{27,28} \mathrm{SiO}_{2}$ particles with diameters across the nanometer length scale until tens of micrometers (see Table 2.1) are prepared with flame pyrolysis and molecular condensation approaches. Unlike SiNPs, the $\mathrm{SiO}_{2}$ NPs' surface is more chemically inert, and these particles are thus readily stored in ambient conditions without significant changes in surface composition.

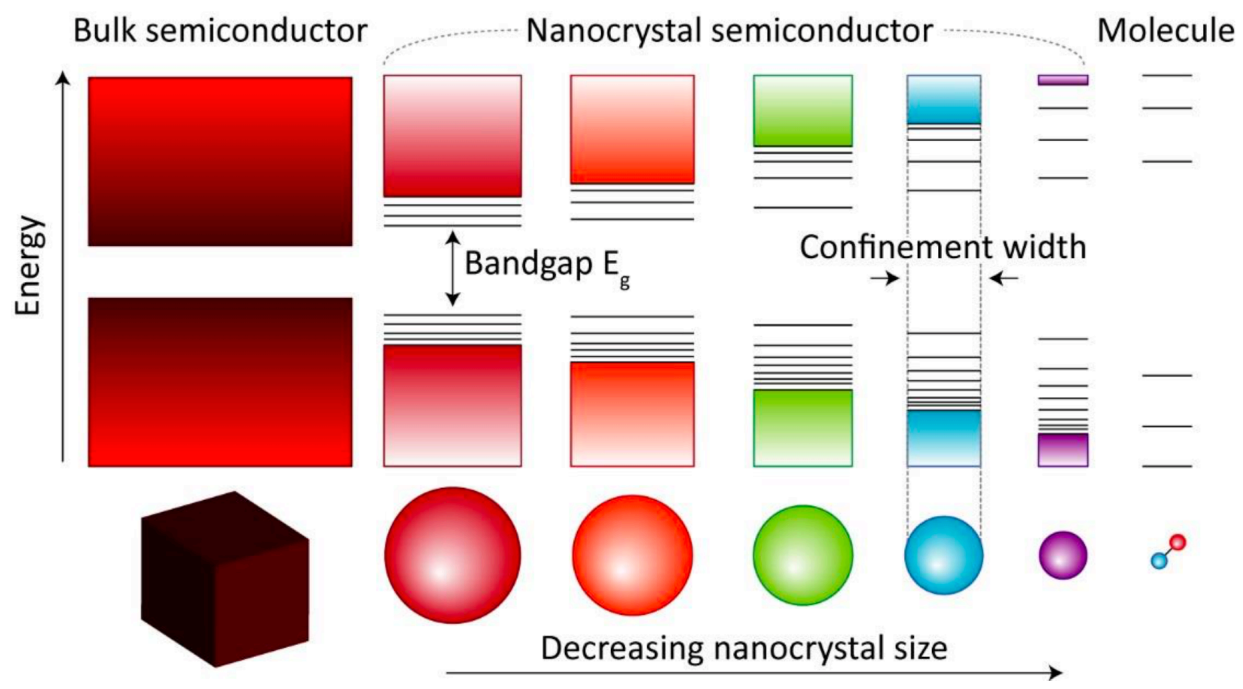

Figure 2.1 The size-dependent bandgaps of bulk material, nanocrystals, clusters, and atoms. $^{26}$

With their low toxicity, good chemical resistance, and optical transparency, $\mathrm{SiO}_{2} \mathrm{NPs}$ are therefore interesting for utilization in drug delivery systems ${ }^{29-31}$, bio-sensors ${ }^{32,33}$, and 
bio-imaging ${ }^{34,35}$. For instance, $\mathrm{SiO}_{2} \mathrm{NPs}$ were reported to deliver drugs to specific locations in the body with a precisely controlled release rate profile. ${ }^{31}$ In addition, organic dye-doped $\mathrm{SiO}_{2} \mathrm{NPs}$ have been extensively investigated as the viable imaging agents for nucleic acids, proteins, and pathogen detection. ${ }^{36-38}$

Before presenting and discussing the emerging Si-containing NP-based applications that are the central topics of the following Chapters of this Thesis, we will first briefly introduce the most common $\mathrm{SiNPs}$ and $\mathrm{SiO}_{2} \mathrm{NP}$ production methods.

\subsection{Silicon-Containing Nanoparticle Production}

During the past few decades, various procedures have been developed to synthesize SiNPs and $\mathrm{SiO}_{2}$ NPs with control over particle size, morphology and composition. ${ }^{39-60}$ These methods can be categorized into either bottom-up or top-down approaches (see Figure 2.2). Si-contaning NPs can be synthesized either as individual particles (primary particles) or as agglomerated particles..$^{39,45,53,60}$ Table 2.1 shows a detailed overview of the most commonly used methods to produce $\mathrm{Si}$ and $\mathrm{SiO}_{2} \mathrm{NPs}$, including their most important characteristics and advantages/disadvantages. Since it is not our scope to review the synthesis of $\mathrm{Si}$ and $\mathrm{SiO}_{2}$ NPs in detail, the interested reader is directed to the references shown in Table 2.1 for a more comprehensive overview.

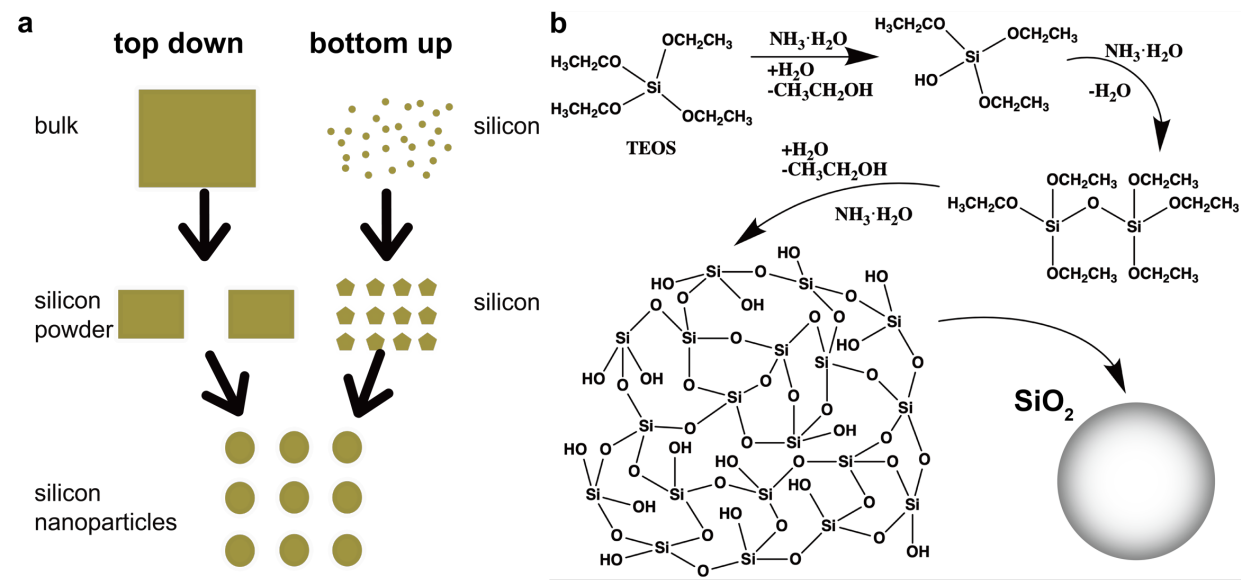

Figure 2.2 a) Top-down and bottom-up approaches for SiNP synthesis; b) schematic of the Stöber reaction for the synthesis of $\mathrm{SiO}_{2}{ }^{61}$

Nowadays, SiNPs can be fabricated via numerous routes, both bottom-up and topdown, with various levels of success. ${ }^{53-60}$ Laser ablation is a physical procedure that has been commonly used to fabricate NPs from their bulk form, including SiNPs. Yoshida et 
al. ${ }^{54}$ reported the preparation of nanometer-sized Si crystallites via excimer laser ablation under helium (He) gas atmosphere. They also found a linear dependence of average particle diameter to the inert gas pressure, which can be used to control the size of SiNPs in the nanometer regime. Ball milling is also a standard procedure to break down bulk materials. ${ }^{9}$ Lam et al. ${ }^{53}$ utilized ball milling of solid graphene with silicon oxide, followed by annealing at $150{ }^{\circ} \mathrm{C}$ to prepare SiNPs. The resulting SiNPs showed a broad size distribution and a $1 \mathrm{~nm}$ silicon oxide overlayer. Chemical procedures have also been applied to obtain SiNPs. Heath ${ }^{57}$ was the first to report the solution-phase synthesis of SiNPs. Heath successfully reduced silicon halides with sodium naphthalenide in a nonpolar organic solvent to produce hydrogen capped free-standing SiNPs. Recently, Hülser et al. ${ }^{60}$ introduced a cost-efficient and scalable synthetic method for SiNPs using a hot-wall reactor. By mixing diborane inside the reactor with silane, they managed to produce boron-doped SiNPs. They managed to achieve a production rate of $0.75 \mathrm{~kg} \mathrm{~h}^{-1}$ and an energy efficiency of $1.4 \mathrm{kWh} \mathrm{kg}^{-1}$ for SiNP fabrication. The developments in large-scale and cost-efficient SiNP synthesis allow SiNPs to be transformed from labscale research to real-life applications. Besides using bulk silicon or Si-containing molecules, there are also reports that present and discuss the use of low-cost, environmentally friendly raw materials like rice husk and wheat straws to produce PL SiNPs. ${ }^{62}$

$\mathrm{SiO}_{2} \mathrm{NPs}$ are mainly prepared via precipitation (precipitated silica), high-temperature flame pyrolysis (fumed silica) or by the molecular condensation of silanol in an aqueous solution and hydrothermal treatments (colloidal silica). ${ }^{39-52}$ Precipitated silica is commonly prepared by adding mineral acid (e.g., $\mathrm{HCl}, \mathrm{H}_{2} \mathrm{SO}_{4}$ ) to the silicate solution. The properties of precipitated silica are strongly dependent on the agitation, $\mathrm{pH}$, temperature, and other environmental parameters. ${ }^{41,42}$ For example, Musić et al. ${ }^{42}$ reported using commercial sodium silicate solution with concentrated $\mathrm{H}_{2} \mathrm{SO}_{4}$ to prepare precipitated silica with a diameter of $15-30 \mathrm{~nm}$. Furthermore, they demonstrated that their approach is ready for large-scale production without altering the properties of the precipitated silica nanoparticles. Fumed silica is typically fabricated at $1,200-1,400{ }^{\circ} \mathrm{C}$ by vapor-phase hydrolysis of silicon tetrachloride $\left(\mathrm{SiCl}_{4}\right)$ in a hydrogen-oxygen flame followed by rapid quenching. ${ }^{63}$ Fumed silica consists of highly coalesced (agglomerated) NPs with a primary particle size ranging from $10-20 \mathrm{~nm} .{ }^{64}$ Recently, Chen et al. ${ }^{40}$ introduced a more economical and environmentally friendly approach for fumed silica synthesis by hydrolysis of $\mathrm{SiCl}_{4}$ with water vapor at a low-temperature range $\left(150{ }^{\circ} \mathrm{C}\right.$ to $250{ }^{\circ} \mathrm{C}$ ). Through this method, they prepared $\mathrm{SiO}_{2} \mathrm{NPs}$ with an average surface area of $418 \mathrm{~cm}^{2} \mathrm{~g}^{-1}$ and an average size of $141.7 \mathrm{~nm}$ at $150{ }^{\circ} \mathrm{C}$. 
Compared to fumed silica, colloidal silica particles synthesized via molecular condensation reactions have a narrower size distribution with almost no coalescence (primary particles), ${ }^{65}$ which is desirable for a wide range of application areas, such as biosensing, bio-imaging, and drug delivery. The most widely used procedure for colloidal $\mathrm{SiO}_{2}$ NPs synthesis is the so-called Stöber reaction. ${ }^{43-47}$ This reaction was first reported by Werner Stöber in $1968 .{ }^{45}$ For a standard Stöber reaction, the particles are formed via a sol-gel process from the hydrolysis and condensation of alkyl silicates in the presence of ammonia as a catalyst (Figure 2.2b). One significant advantage of the Stöber reaction, as mentioned, is the ability to produce nearly monodisperse $\mathrm{SiO}_{2} \mathrm{NPs}$. In addition, the reaction can be easily scaled up and the products are readily transferred to aqueous solutions. ${ }^{45}$ Besides the Stöber reaction, other methods for the preparation of colloidal $\mathrm{SiO}_{2} \mathrm{NPs}$ have been introduced. For example, Gorji et al. ${ }^{66}$ reported a synthetic procedure for the preparation of $\mathrm{SiO}_{2}$ NPs using tetraethyl orthosilicate (TEOS), polyethylene glycol, and hydrochloric acid. In addition, Zulfiqar et al. ${ }^{67}$ demonstrated the fabrication of $\mathrm{SiO}_{2}$ NPs using bentonite clay as the primary silica source. They successfully fabricated $\mathrm{SiO}_{2}$ NPs ranging from $70 \mathrm{~nm}$ to $1.4 \mu \mathrm{m}$ in diameter through the thermal treatment of the bentonite clay in ethanol in the presence of nitric acid.

Having introduced $\mathrm{Si}$ and $\mathrm{SiO}_{2}$ NPs as an interesting class of materials, and shortly reviewing their common production methods, we will focus in the remainder of this Chapter on exciting, emerging, and in our opinion, overlooked application areas of these NPs. Thus, in the following sections we present and discuss the frontiers of Si-containing NPs research and review the utilization of Si-containing NPs as ink for printable electronics (2.3), in biomedical applications (2.4), as nucleation agents for polymer foam cell nucleation (2.5), and as catalysts for chemical reactions (2.6). 
Table 2.1 List of selected Si-containing NPs synthesis approaches with their advantages/disadvantages.

\begin{tabular}{|c|c|c|c|c|c|}
\hline Method & Material & Temperature & Diameter (nm) & Advantage & Disadvantage \\
\hline & & & $\mathrm{SiO}_{2} \mathrm{NP}$ & & \\
\hline $\begin{array}{c}\text { flame } \\
\text { pyrolysis }^{39}\end{array}$ & $\mathrm{SiCl}_{4}$, methane, propane & $1500^{\circ} \mathrm{C}$ & & \multirow{2}{*}{$\begin{array}{l}\text { High reaction speed, } \\
\text { hydrophilic surface }\end{array}$} & \multirow{2}{*}{$\begin{array}{l}\text { Particles are } \\
\text { agglomerated }\end{array}$} \\
\hline $\begin{array}{c}\text { flame } \\
\text { pyrolysis }^{40}\end{array}$ & $\mathrm{SiCl}_{4}, \mathrm{~N}_{2}, \mathrm{H}_{2} \mathrm{O}$ & $125-300^{\circ} \mathrm{C}$ & $\begin{array}{c}6.5-8.2(\mathrm{P}) \\
141.7-186.1(\mathrm{~A})\end{array}$ & & \\
\hline $\begin{array}{l}\text { precipitated } \\
\text { silica }^{41}\end{array}$ & silica gel, $\mathrm{HCl}, \mathrm{NaOH}, \mathrm{H}_{2} \mathrm{SO}_{4}$ & RT & $50 \mathrm{~nm}(\mathrm{M})$ & \multirow{2}{*}{ High solid content } & \multirow{2}{*}{$\begin{array}{l}\text { Long reaction time, } \\
\text { corrosive substances. }\end{array}$} \\
\hline $\begin{array}{l}\text { precipitated } \\
\text { silica }^{42}\end{array}$ & sodium silicate, $\mathrm{H}_{2} \mathrm{SO}_{4}$ & $80-90{ }^{\circ} \mathrm{C}$ & $15-30 \mathrm{~nm}(\mathrm{M})$ & & \\
\hline $\begin{array}{l}\text { modified } \\
\text { Stöber }^{43}\end{array}$ & TEOS, $\mathrm{NH}_{3}, \mathrm{H}_{2} \mathrm{O}$, isopropanol & $5,20^{\circ} \mathrm{C}$ & $30-930(\mathrm{M})$ & High solid content & \\
\hline $\begin{array}{l}\text { modified } \\
\text { Stöber }^{44}\end{array}$ & $\begin{array}{l}\text { TEOS, } \mathrm{NH}_{3}, \mathrm{H}_{2} \mathrm{O} \text {, ethanol } \\
\text { (DMSO), Alexa Fluor } 555\end{array}$ & RT & $13-64(\mathrm{M})$ & Adjustable luminescence & \\
\hline Stöber ${ }^{45}$ & TEOS, $\mathrm{NH}_{3}, \mathrm{H}_{2} \mathrm{O}$, alcohols & RT & $50-2000(\mathrm{M})$ & \multirow{3}{*}{$\begin{array}{l}\text { Controllable size, short } \\
\text { reaction time, good } \\
\text { reproducibility }\end{array}$} & \\
\hline Stöber ${ }^{46}$ & TEOS, $\mathrm{NH}_{3}, \mathrm{H}_{2} \mathrm{O}$, ethanol & $30^{\circ} \mathrm{C}$ & $160-600(\mathrm{M})$ & & \\
\hline $\begin{array}{c}\text { seeded } \\
\text { growth }^{47}\end{array}$ & TEOS, $\mathrm{NH}_{3}, \mathrm{H}_{2} \mathrm{O}$, ethanol & $10,25,40^{\circ} \mathrm{C}$ & Up to 2 um $(\mathrm{M})$ & & \\
\hline
\end{tabular}




\begin{tabular}{|c|c|c|c|c|c|}
\hline & & & porous $\mathrm{SiO}_{2} \mathrm{NP}$ & & \\
\hline sol-gel ${ }^{48}$ & $\begin{array}{c}\text { TEOS, CTAB, CPC, ethanol, } \\
\mathrm{NH}_{3}\end{array}$ & RT & $\begin{array}{c}400-1100 \text { (particle) } \\
2.81-32.8 \text { (pore) }\end{array}$ & \multirow{3}{*}{$\begin{array}{l}\text { Controllable pore size and } \\
\text { geometry, short reaction } \\
\text { time }\end{array}$} & \multirow{5}{*}{$\begin{array}{l}\text { Surfactants are hard } \\
\text { to remove, usually } \\
\text { requires long-time } \\
\text { calcination }\end{array}$} \\
\hline sol-gel ${ }^{49}$ & $\begin{array}{l}\text { TEOS, } \mathrm{C}_{14} \mathrm{CluA}, \mathrm{NaOH}, \mathrm{TMAPS} \\
\text { methanol, } \mathrm{H}_{2} \mathrm{O}\end{array}$ & $60^{\circ} \mathrm{C}$ & $2.2-4.7$ (pore) & & \\
\hline sol-gel $5^{50}$ & $\begin{array}{l}\text { TEOS, } \mathrm{NH}_{3}, \mathrm{H}_{2} \mathrm{O}, \mathrm{HAD} \\
\quad \text { isopropanol }\end{array}$ & $\mathrm{RT}$ & & & \\
\hline sol-gel ${ }^{51}$ & TMOS, Pluronic P123, HCl & $35^{\circ} \mathrm{C}$ & $\begin{array}{c}500-2000 \text { (particle) } \\
4 \text { (pore) }\end{array}$ & \multirow{2}{*}{$\begin{array}{l}\text { No organic solvent, rod- } \\
\text { like/hexagonal prisms } \\
\text { particles }\end{array}$} & \\
\hline sol-gel $\left.\right|^{52}$ & $\begin{array}{l}\text { TEOS, Pluronic } \mathrm{P} 123 \text {, citric acid, } \\
\text { trisodium citrate, } \mathrm{H}_{2} \mathrm{O}\end{array}$ & RT & $\begin{array}{l}500 \text { (particle) } \\
5.8-9.8 \text { (pore) }\end{array}$ & & \\
\hline \multicolumn{6}{|c|}{ SiNP } \\
\hline ball milling ${ }^{53}$ & Graphite, $\mathrm{SiO}_{2}$ powder & RT & 7 & Low cost, scalable & \\
\hline laser ablation $^{54}$ & Single crystalline Si wafer & RT & 10 & Single crystalline SiNPs & \\
\hline etching $^{55}$ & $\begin{array}{l}\text { Si wafer, HF, ethanol, } \\
\text { isopropanol, allylamine, } \\
\mathrm{H}_{2} \mathrm{PtCl}_{6}\end{array}$ & RT & $4.6 \pm 1.9$ & Resistant to aging & $\begin{array}{l}\text { Typically have a } \\
\text { broad size distribution } \\
\text { and require an }\end{array}$ \\
\hline
\end{tabular}




\begin{tabular}{|c|c|c|c|c|c|}
\hline inverse & $\mathrm{SiX} 4(\mathrm{X}=\mathrm{Cl}, \mathrm{Br}, \mathrm{I})$ & RT & $1.8-10$ & \multirow{3}{*}{ Solution-based } & \multirow{6}{*}{$\begin{array}{l}\text { Some of the methods } \\
\text { require high } \\
\text { temperature and } \\
\text { vacuum }\end{array}$} \\
\hline micelle & & & & & \\
\hline hydrothermal ${ }^{57}$ & $\begin{array}{c}\mathrm{SiCl}_{4}, \mathrm{HSiCl}_{3} / \mathrm{C}_{8} \mathrm{H}_{17} \mathrm{SiCl}_{3}, \mathrm{Na}, \\
\text { toluene, hexane }\end{array}$ & $385^{\circ} \mathrm{C}$ & 5 & & \\
\hline $\begin{array}{l}\text { microwave } \\
\text { pyrolysis }^{58}\end{array}$ & $\mathrm{SiH}_{4}, \mathrm{H}_{2}, \mathrm{Ar}$ & RT & $6-11$ & Single crystalline & \\
\hline laser pyrolysis ${ }^{59}$ & $\mathrm{SiH}_{4}$ & $\mathrm{RT}$ & $2-7$ & Single crystalline & \\
\hline $\begin{array}{c}\text { hot-wall } \\
\text { pyrolysis }^{60}\end{array}$ & Silane, $\mathrm{H}_{2}, \mathrm{~N}_{2}$ & $800-100^{\circ} \mathrm{C}$ & $150-450$ & Low cost, scalable & \\
\hline
\end{tabular}

* RT - room temperature; (M) - monodisperse; (P) - primary particle size; (A) - agglomerate size; TMAPS - Ntrimethoxylsilylpropyl-N,N,N-trimethyl-ammonium chloride; CTAB - n-Hexadecyltrimethylammonium bromide; $\mathrm{C}_{14} \mathrm{CluA}$ - Nmyristoyl-L-glutamic acid; TEOS - tetraethyl orthosilicate; TMOS - tetramethyl orthosilicate; $\mathrm{C}_{8} \mathrm{H}_{17} \mathrm{SiCl}_{3}$ - n-octyltrichlorosilane; $\mathrm{HAD}$ - n-hexadecylamine. 


\subsection{Silicon Nanoparticle-Based Electronic Ink}

SiNPs are a promising candidate for the fabrication of low-cost, large-area, lightweight, flexible electronic devices fabricated by printing techniques. Therefore, section 2.3.1. will briefly introduce inkjet printing technology, set forth the requirements of SiNPs based electronic inks, and continue with an in-depth presentation of the oxidative stability (2.3.2), colloidal stability (2.3.3), and printing of SiNPs (2.3.4).

\subsubsection{Inkjet Printing}

Inkjet printing is considered to be a potential replacement for lithography by electronic component manufacturers. ${ }^{68,69}$ It can produce electronic devices on polymeric substrates or even on paper with improved quality, production speed, and cost-efficiency compared to traditional lithography approaches. ${ }^{70,71}$ The inkjet printing of electronic devices typically involves two steps. The first step is depositing and drying inks to form thin films/conductive patterns. The second is an annealing/sintering step to modify the printed layer microstructure and adjust electrical conductivity. ${ }^{68}$ Inkjet printing is considered to be an advanced and economical approach to manufacturing electronic devices. However, as for any printing technique, the most critical factors for success lie on the ink end. The quality and reproducibility of the inkjet printing process rely on the control of drop formation and the interaction between the deposited drops and substrates. In the last decade metal NPs, ${ }^{72,73}$ conductive polymers, ${ }^{74}$ and organic-metal hybrids ${ }^{75}$ have been extensively studied as conductive inks for inkjet printing of electronic devices.
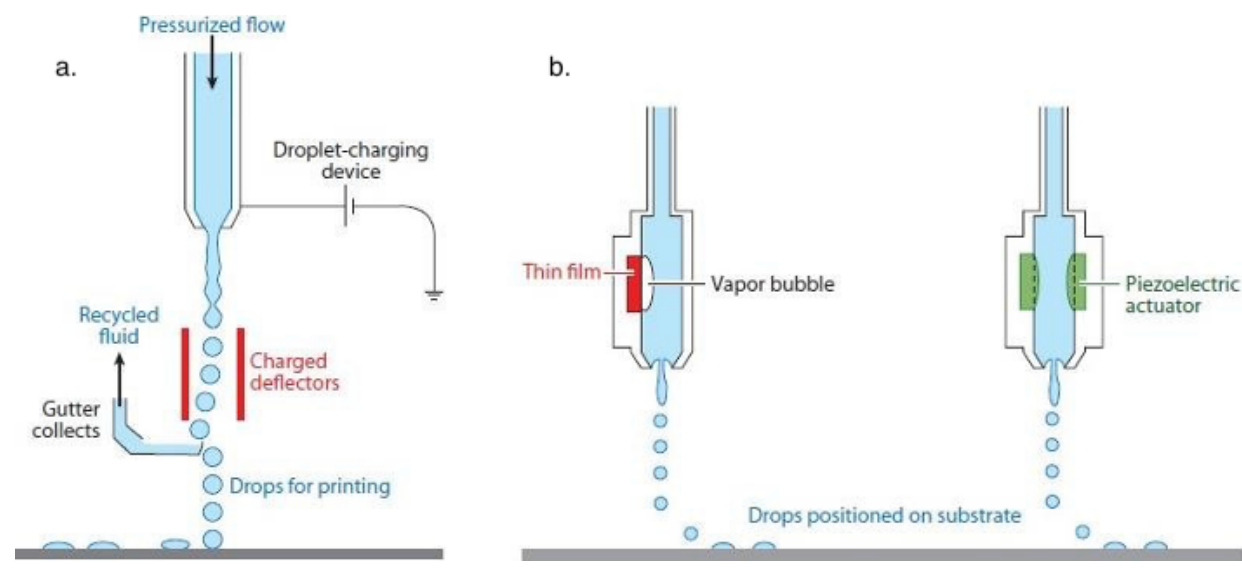

Figure 2.3 Schematic drawing of a) continuous, b) thermal, and c) piezoelectric drop on demand inkjet printers. ${ }^{76}$

Based on the mechanism of drop formation, inkjet printing can be divided into two 
categories: drop on demand (DoD) and continuous inkjet (CIJ) printing (Figure 2.3) ${ }^{68}$ The drop diameters for DoD and CIJ are in the range of 20-50 $\mu \mathrm{m}$ and $100 \mu \mathrm{m}$, respectively. The nozzle of a CIJ printer head continuously produces droplets. A charged deflector deflects the unwanted droplets for collection and allowing recycling to avoid ink waste. (We note that there is a risk that the ink gets polluted/altered during ink recycling when exposed to the atmosphere.) The advantage of CIJ printing is that through continuous jetting, the nozzles are continuously kept at a high moisture level, which helps to prevent undesirable nozzle clogging. In DoD printing, a droplet is only ejected from the nozzle when the pressure pulse propagating the ink reaches a certain threshold. This pressure pulse is usually generated by a current pulse that either boils a small portion of the ink in the nozzle chamber or introduces mechanical force to squeeze the chamber through a piezoelectric transducer. ${ }^{68}$ As such, the ink will only be released from the nozzle when needed. This avoids wasting ink and the unnecessary exposure of ink to ambient conditions, which may lead to ink pollution and/or degradation. One of the major disadvantages of DoD printing is the lower production speed compared to CIJ printing.

Nowadays, inkjet printing has been successfully utilized to fabricate various electronic devices like flexible circuits ${ }^{77}$, memory devices ${ }^{78}$, displays ${ }^{79,80}$, sensors ${ }^{81-83}$, field-effect transistors $(\mathrm{FET})^{84-88}$, batteries ${ }^{89}$, and solar cells ${ }^{90,91}$. For example, Sekitani et al. ${ }^{88}$ used an inkjet printer to manufacture an organic thin-film transistor. Using a subfemtoliter inkjet printer, they fabricated $\mathrm{p}$-channel and n-channel transistors with single-micrometer resolution on rigid and flexible substrates that can operate with a very low voltage $(3 \mathrm{~V})$. Molina-Lopez et al. ${ }^{87}$ successfully utilized inkjet printing to build stretchable FET arrays and interconnections (see Figure 2.4). They used poly(3,4ethylenedioxygthiophene)-poly(styrenesulfonate) (PEDOT:PSS) as the source/drain/gate electrodes and interconnects, semiconducting single-walled carbon nanotubes (SCSWCNTs) as the semiconductive channel, and poly(vinylidene fluoride-cohexafluoropropylene) (PVDF-HFP) as the gate dielectric. They demonstrated that their printed FETs were able to operate at sub-volt conditions with the capability of maintaining electrical performance up to $20 \%$ applied strains. 


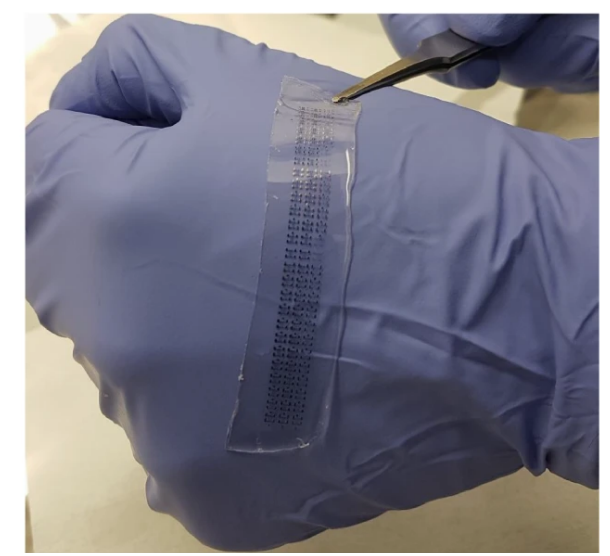

Figure 2.4 Photo of a printed flexible transistor array bent over a hand. ${ }^{87}$

The examples presented above clearly demonstrate the potential of inkjet printing as enabling technology for the mass production of electronic devices, but like mentioned before, its success is highly dependent on the development of the required inks. The influence of physical parameters like ink viscosity, density, and surface tension on the droplet formation and ink-substrate interactions, are well reported in the open literature. ${ }^{92}$ An additional parameter that requires attention is the long-term stability of the electronic inks. This is considered a pivotal property to utilize inkjet printing as a tool in the industrial manufacturing of electronic devices/components. Therefore, the remainder of this section will provide a comprehensive discussion of factors related to long-term Sicontaining ink stability, such as the aging behavior and the colloidal stability of SiNPs, as one of the first steps towards formulating SiNP-based inks for inkjet printing of functional electronic devices.

\subsubsection{Surface Oxidation of Silicon Nanoparticles}

Most studies reporting on inducing long-term stability to NP suspensions can be categorized as using either steric stabilization or electrostatic stabilization approaches. ${ }^{93,94}$ For the steric stabilization approach, polymeric molecules are adsorbed onto or bonded to a NP's surface to create steric repulsion (see Figure 2.5a). When the distance between two particles is less than twice the polymer layer thickness, the adsorbed/grafted polymers will generate a repulsive entropic force against each other and suppress van der Waals force-induced particle agglomeration. ${ }^{94}$ Electrostatic stabilization is another effective method to overcome van der Waals attraction between particles. ${ }^{94}$ It is based on the principle that the same sign electrically charged particles experience a mutual repulsive force. For the electrostatic stabilization approach, ionic groups are 
adsorbed on the particle surface. Counter ions, introduced to the system to maintain electroneutrality, surround the NPs and form electrical double layers. When the electric potential of the double layer is sufficiently high, the van der Waals attraction between particles is suppressed by Coulombic repulsion (see Figure $2.5 \mathrm{~b}$ ).
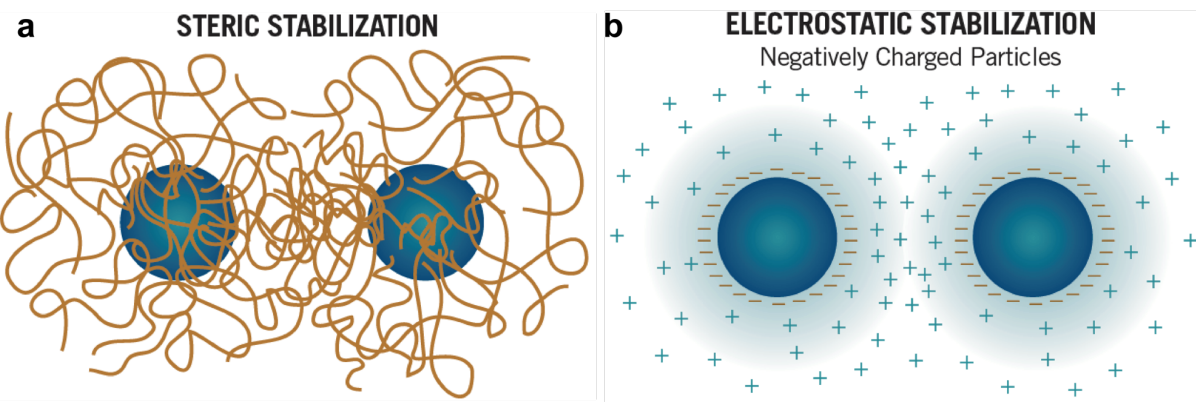

Figure 2.5 Schematic of a) steric stabilization and b) electrostatic stabilization. The image was adapted from reference ${ }^{95}$.

To successfully exploit the stabilization mechanisms on SiNP dispersions, we must first understand the surface chemistry of these particles in their initial and aged states. Upon formation, the surface of SiNPs mainly consists of Si-H and Si-Si bonds. ${ }^{96}$ Both these bonds are strong reducing agents that are able to reduce water to hydrogen while forming a surface exposed $\mathrm{SiO}_{2}$ layer at ambient conditions (see eq. 1-4).

$$
\begin{aligned}
& \mathrm{SiH}_{4}+2 \mathrm{H}_{2} \mathrm{O} \rightarrow \mathrm{SiO}_{2}+8 e^{-}+8 \mathrm{H}^{+} \\
& \mathrm{SiH}_{4}+2 \mathrm{H}_{2} \mathrm{O} \rightarrow \mathrm{SiO}_{2}+4 \mathrm{H}_{2} \quad \text { eq. } 2 \\
& \mathrm{Si}+2 \mathrm{H}_{2} \mathrm{O} \rightarrow \mathrm{SiO}_{2}+4 e^{-}+4 \mathrm{H}^{+} \quad \text { eq. } 3 \\
& \mathrm{Si}+2 \mathrm{H}_{2} \mathrm{O} \rightarrow \mathrm{SiO}_{2}+2 \mathrm{H}_{2} \quad \text { eq. } 4
\end{aligned}
$$

In addition to $\mathrm{SiO}_{2}$ formation, oxidation of $\mathrm{Si}-\mathrm{Si}$ bonds at ambient conditions can lead to the generation of new Si-H species exposed at the SiNPs surface (see Figure 2.6). ${ }^{11}$ First, a surface $\mathrm{Si}$ atom is attacked by a nucleophile $\left(\mathrm{OH}^{-}\right)$to produce a 5-coordinate $\mathrm{Si}$ intermediate, followed by $\mathrm{Si}-\mathrm{Si}$ bond cleavage and $\mathrm{Si}-\mathrm{O}$ bond formation. The Si-O surface-exposed layer prevents further oxidation of the $\mathrm{Si}$-Si species beneath the surface. However, at higher $\mathrm{pH}$ values (i.e. $>7$ ), the exposed $\mathrm{Si}-\mathrm{O}$ bonds disassociate in the aqueous solution, resulting in further, or even complete, oxidation/dissolution of the SiNPs. ${ }^{97}$ For instance, at elevated temperature Si wafers are readily etched in e.g. $10 \mathrm{wt} \%$ $\mathrm{KOH}$ solutions within tens of seconds to few minutes, depending on the crystallinity of the Si wafer. ${ }^{98,99}$ We note that based upon our experimental observations, SiNPs with a 
diameter around $50 \mathrm{~nm}$ were dissolved entirely in less than 10 minutes when exposed to similar conditions (i.e. $5 \mathrm{wt} \% \mathrm{KOH}$ ), resulting in the formation of silicic acid species. ${ }^{100}$

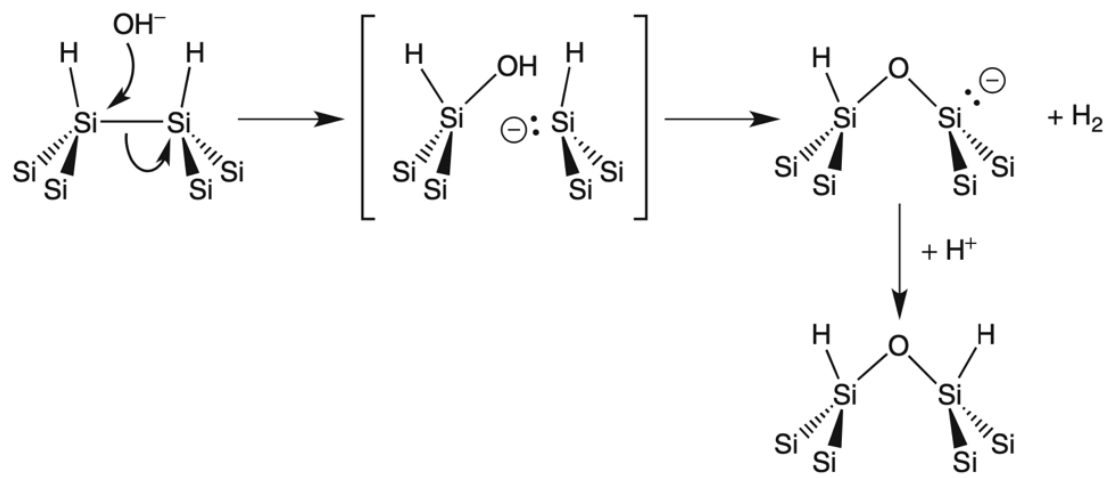

Figure 2.6 Schematic of the oxidation process for $\mathrm{Si}-\mathrm{Si} / \mathrm{Si}-\mathrm{O}$ bonds in an aqueous environment. ${ }^{96}$

Furthermore, Si-H and Si-Si species are also sensitive to oxygen. The oxidation products of these species depend on the oxidation temperature. ${ }^{5}$ Since the Si-Si bond (bond energy $255 \mathrm{~kJ} \mathrm{~mol}^{-1}$ ) is weaker than the $\mathrm{Si}-\mathrm{H}$ bond (bond energy $395 \mathrm{~kJ} \mathrm{~mol}^{-1}$ ), the oxidation of SiNPs at room temperature tends to form an overlayer of $\mathrm{SiO}_{2}$ with the presence of surface-exposed Si-H bonds (Figure 2.7a).$^{96}$ These Si-H species can exist for months or even longer when stored in air at ambient conditions. The long lifetime of Si$\mathrm{H}$ species in air opens the possibility of modifying SiNPs with hydrosilylation, which is one of the most commonly used techniques to attach unsaturated bonds to a Si surface. We will discuss hydrosilylation later in more detail (see Section 2.3.3).

When raising the temperature to $60-100{ }^{\circ} \mathrm{C}$, the oxidation process will break the Si$\mathrm{H}$ and the Si-Si bonds located at the SiNP surface, resulting in a surface with exposed Si$\mathrm{O}$ and $\mathrm{Si}-\mathrm{OH}$ bonds (Figure 2.7b). ${ }^{101}$ At temperatures above $200{ }^{\circ} \mathrm{C}$ and in the presence of oxygen, the whole skeleton of the SiNPs is converted to silicon dioxide (Figure 2.7c). The time scale of this transformation depends on the type of SiNPs and the oxidation temperature. Microporous and macroporous SiNPs need approximately one hour and three hours, respectively, to achieve complete oxidation. ${ }^{96}$ 


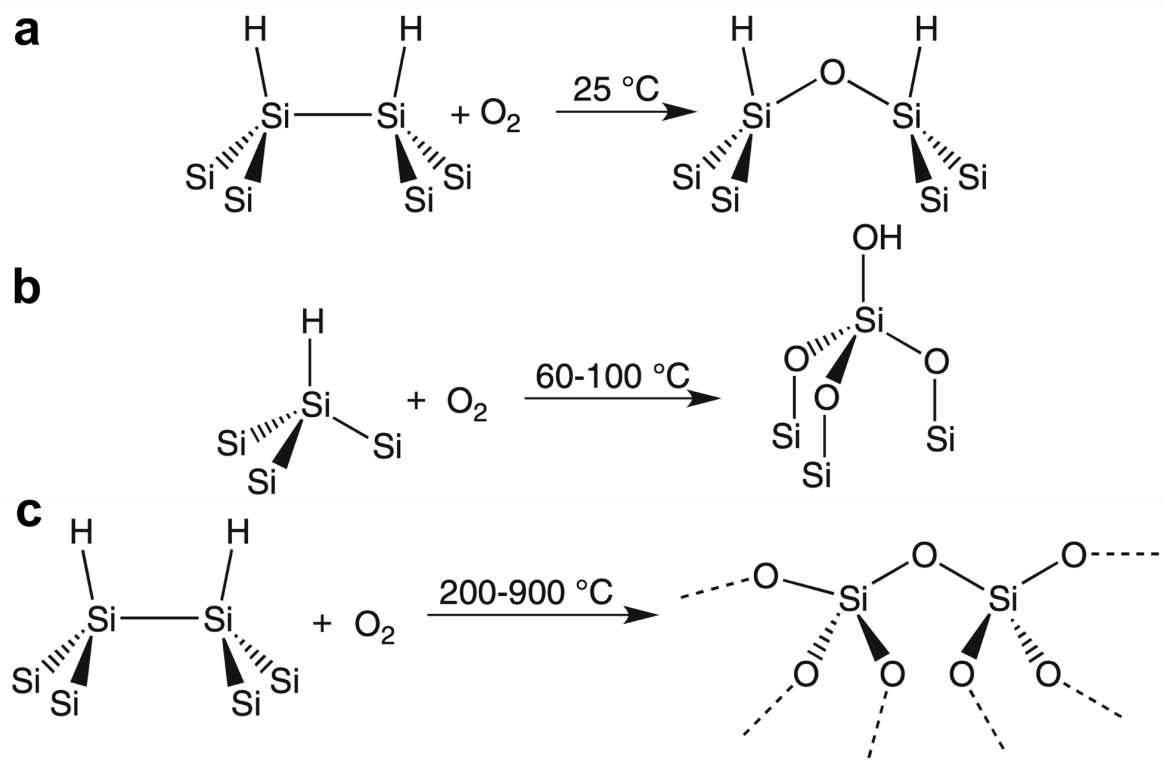

Figure 2.7 Schematic of the oxidation process for Si-Si/Si-O bond in the oxygencontaining atmosphere for various temperatures. ${ }^{96}$

We note that SiNPs can also undergo chemical and electrochemical oxidation that is considered unlikely to occur during the storage of aqueous SiNP dispersions. As such, it is not expected to influence the long-term stability of Si NP-based inks. Therefore, we will not discuss the chemical and electrochemical oxidation of SiNPs in this Chapter. Instead, the interested reader is directed to references ${ }^{96,102,103}$.Having established a comprehensive understanding of the surface chemistry of SiNPs, the following section introduces strategies to stabilize SiNP suspensions in more detail.

\subsubsection{Surface Modification of Silicon Nanoparticles to Fabricate Stable Colloidal Inks}

The complexity of the SiNPs' surface chemistry (see section 3.2) makes it hard to estimate their colloidal stability when solvents are changed during ink formulation. Also, highly active surface moieties like $\mathrm{Si}-\mathrm{H}$ and $\mathrm{Si}-\mathrm{O}$ can cause unpredicted complications upon interacting with solvents (see Chapter 3), especially when considering long-term colloidal stability. Therefore, it is common practice to use surface modification strategies to protect the SiNPs, alter their dispersion stability, and even introduce new properties. Numerous efforts have been made to obtain long-term stability of SiNP inks. ${ }^{104-108}$

As mentioned before, hydrosilylation is widely accepted as an efficient approach to modify surface-exposed Si-H groups. By reacting Si-H with double or triple C-C bonds 
in the presence of heat, ${ }^{109}$ light, ${ }^{110}$, or Lewis acid catalyst, ${ }^{111} \mathrm{Si}-\mathrm{C}$ bonds can be formed on the surface of SiNPs. These surface-exposed $\mathrm{Si}_{-} \mathrm{CH}_{2}-\mathrm{X}$ moieties have demonstrated excellent stability in aqueous environments compared to $\mathrm{Si}-\mathrm{OC}(\mathrm{O}) \mathrm{CH}_{2}$ - bonds. ${ }^{112}$ This is ascribed to the low electronegativity of the carbon atom compared to the oxygen atom. Thus, the $\mathrm{Si}-\mathrm{C}$ is less susceptible to associative attacks by nucleophiles. Hydrosilylation allows one to introduce various organic functional groups to the SiNP surface, for example, carboxylic acid, ester, and ether, which opens up possibilities for further reactions. However, the high sensitivity of hydrosilylation reactions to oxygen and water, and the commonly obtained relatively low surface coverage of functional groups, especially for alkyne-driven hydrosilylation, limit their use for the large-scale surface modification of SiNPs. ${ }^{113-115}$

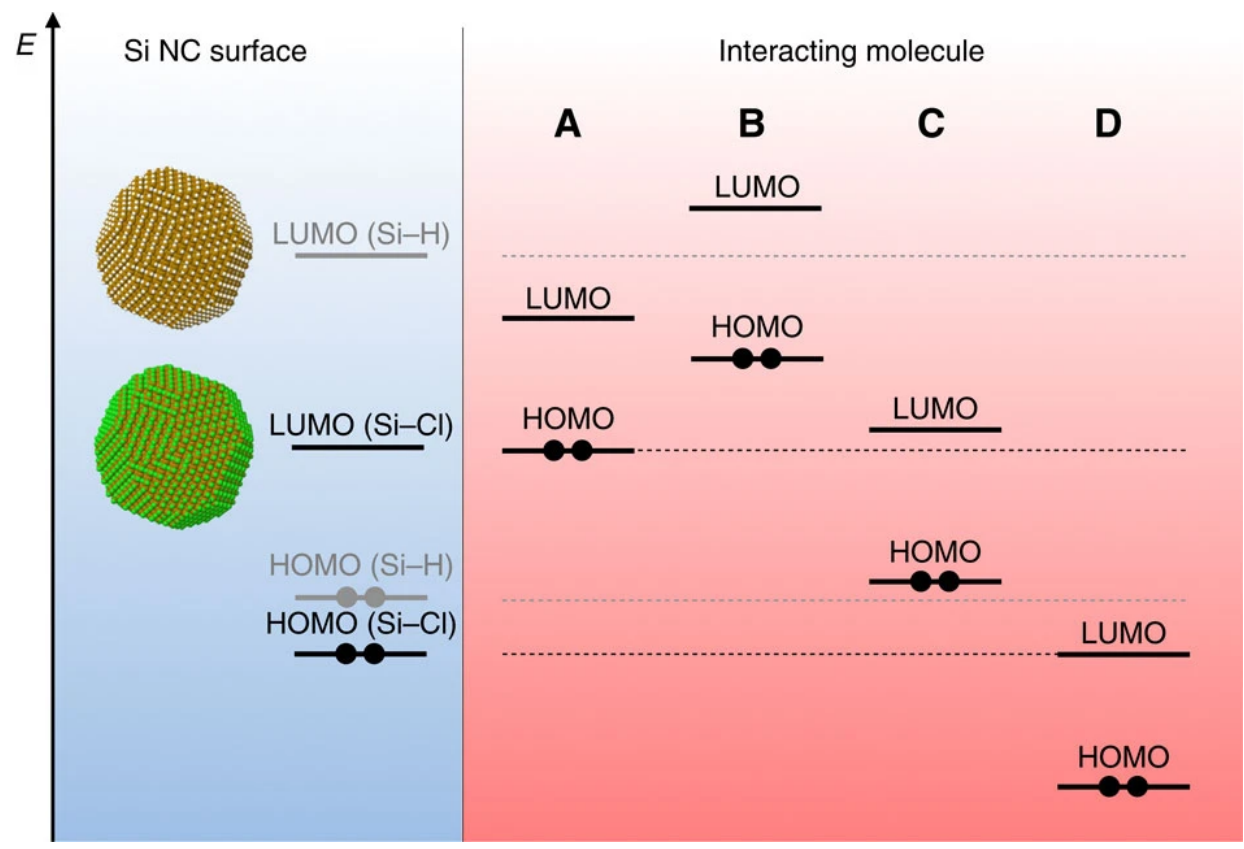

Figure 2.8 Schematic of the hypervalent surface interaction of silicon nanocrystals. The Cl terminated Si NCs have a lower LUMO energy compared to H terminated Si NCs. Molecule A represents a molecule with favorable energetic alignment for hypervalent interactions. B molecules are strong Lewis base donors, such as dimethylformamide and pyridine. C molecules are chemically inert molecules, such as hydrocarbon and chlorinated hydrocarbon solvents. D molecules represent protic solvents, such as alcohols containing acidic hydrogens, which hydrolyze the Si-Cl group. HOMO, highest occupied molecular orbital; LUMO, lowest unoccupied molecular orbital. ${ }^{104}$

Another approach to enhance the colloidal stability of SiNPs without binding carbon atoms/molecules to the NPs includes careful tuning of their surface composition. Holman 26 
et al. ${ }^{116}$ reported the successful preparation of stable colloidal suspensions of $\mathrm{H}$ - and $\mathrm{Cl}$ terminated germanium $(\mathrm{Ge})$ nanocrystals $(\mathrm{NCs})$ in benzonitrile. Erogbogbo et al. ${ }^{117}$ prepared stable colloidal suspensions of $\mathrm{Ge}, \mathrm{Si}$, and $\mathrm{Ge} / \mathrm{Si}$ alloy (Sil-xGex) NCs in various solvents. They first prepared pure $\mathrm{Si}$, Ge NPs, and alloyed NPs, subsequently, the NPs were dispersed in both benzonitrile and acetonitrile using extensive sonication without the need for any subsequent surface modification or ion addition. These organic surfactant-free NP inks possess the potential for the low-cost fabrication of functional optoelectronic, thermoelectric and photovoltaic devices. Another successful example of the preparation of stable free-standing colloidal Si nanocrystals (Si NCs) was presented by Kůsová et al. ${ }^{106}$ They used extended stirring of oxygen passivated PL Si NCs in xylene and showed that passivation of the particle surface resulted in long-term stability while maintaining the PL property. These PL colloidal Si NCs could be a valuable candidate in biological labeling and colloidal LED. Wheeler et al ${ }^{104}$ reported the formation of freestanding Si NCs and successfully exploited the Cl-termination of Si NCs surfaces to readily engage in hypervalent interactions with hard donor molecules to form a stable colloidal suspension (see Figure 2.8). By terminating the Si NCs' surface with $\mathrm{Cl}$, the acidic Si NCs' surface favorably interacts with donor solvent molecules to create a layer of hard donor molecules immobilized around the particle surface. These thin layers of donor molecules around the Si NCs help to avoid direct contact between particles, therefore preventing agglomeration. By exploiting the hypervalent interaction between the Si NCs and solvent molecules, they successfully produced stable Si NC suspension without using ionic ligands or large organic molecules that could potentially hinder charge transport in the fabricated silicon devices. However, the preparation of freestanding SiNP suspensions by these methods typically requires large amounts of volatile solvents, low reactant concentrations (ppm level), employing (unstable) surface functional groups and typically yield small particle sizes (typically below $10 \mathrm{~nm}$ ). ${ }^{105,107,119}$ These requirements and small Si-containing particle size limit the widespread application of SiNP dispersions in large-scale industrial applications, such as printing conductive patterns to fabricate functional electrical devices. ${ }^{68}$

Grafting of polymers to the particle surface represents another efficient way to enhance the colloidal stability of NP dispersions. ${ }^{19,120}$ Depending on the type of polymer, grafting can either increase electrostatic repulsion, decrease van der Waals forces, or result in severe steric hindrance to avoid undesirable particle aggregation. ${ }^{121}$ In general, there are three methods to anchor polymer chains onto a (particle) surface, i.e. "grafting to", "grafting through", and "grafting from" (see Figure 2.9). ${ }^{122}$ The "grafting to" method is a classical approach to fabricate polymer-particle hybrids. ${ }^{122}$ A standard "grafting to" procedure involves the synthesis of polymers and NPs separately, followed by the 
attachment of the polymer chains onto the surface of the particles. For the "grafting to" method, both the polymer molecular weight $\left(\mathrm{M}_{\mathrm{n}}\right)$ and the polydispersity index (PDI) can be characterized before polymer attachment, which is not possible for the grafting through and grafting from methods (see below). The disadvantage of the "grafting to" method is that typically, low grafting densities are obtained, caused by the steric hindrance of the already attached polymer chains acting on the chains approaching the partly covered substrates. ${ }^{123}$

"Grafting through" and "grafting from" have in common that polymers are attached to the particle surface during their polymerization. ${ }^{124,125}$ For the grafting through approach, also known as the "macromonomer" method, polymerizable units such as vinyl units are first immobilized at the particle surface (i.e the macromonomer). Then, depending on the employed polymerization method, monomers, initiators, catalysts, complexing agents are added to the particle suspension to start the polymerization process. During chain propagation, polymerizable units on the particle surface get incorporated in the backbone of the propagating chains, leading to propagating chains anchored onto the particle surface. In this manner, a polymer overlayer on the particle is formed. To date, the mechanism of the "grafting through" method is still not fully understood, even though it has been widely used in scientific research and industrial applications. ${ }^{126}$ In the "grafting from" method, also known as surface-initiated polymerization, functional initiators are covalently attached to the particle surface. In the subsequent step, these initiators are used to initiate polymerization. Due to the nature of this process, "grafting from" tends to give the lowest steric hindrance during polymerization, which results in relatively high grafting densities.

Among the polymerization techniques used for the "grafting from" method, surfaceinitiated controlled radical polymerization (SI-CRP) is considered one of the most effective approaches to synthesizing polymer-particle hybrids. ${ }^{127-129}$ In general, there are three controlled radical polymerization (CRP) methods that have been extensively studied: atom transfer radical polymerization (ATRP) $)^{130,131}$, reversible additionfragmentation chain transfer (RAFT) polymerization ${ }^{132,133}$, and nitroxide-mediated polymerization (NMP) ${ }^{134-136}$. The ATRP, RAFT, and NMP methods each have their specific requirements regarding available monomers, functional initiators, and polymer architectures. Compared to RAFT and NMP, ATRP has much more tolerance to functional groups and reaction conditions. ${ }^{130}$ Most importantly, the initiators, transition metal catalysts, and ligands used for ATRP are commercially available. Due to these advantages, surface-initiated ATRP has been extensively investigated for NP surface modification. Therefore in this Chapter, we will briefly introduce surface-initiated ATRP 
and present some highlights for Si-containing NP modification via SI-ATRP. Readers interested in RAFT and NMP are directed to ${ }^{132,133}$ (RAFT) and ${ }^{134-136}$ (NMP) for more detailed information.
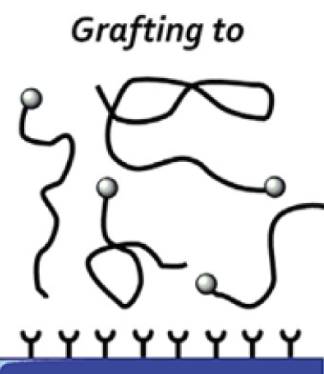

Substrate to be grafted

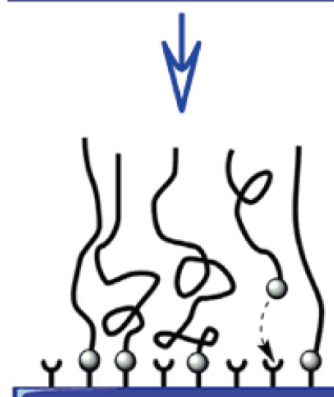

Substrate to be grafted
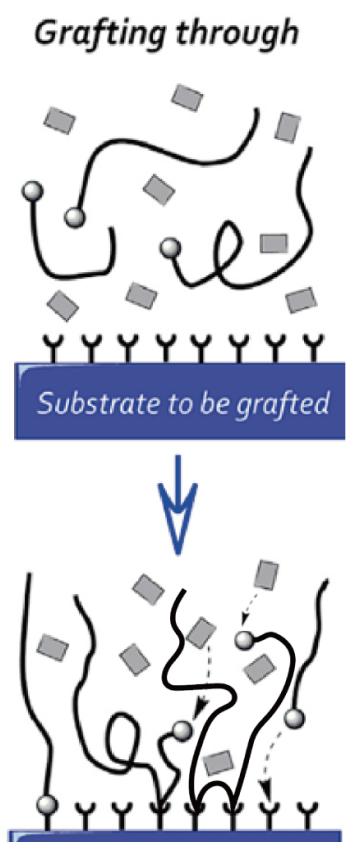

Substrate to be grafted

\section{Grafting from}

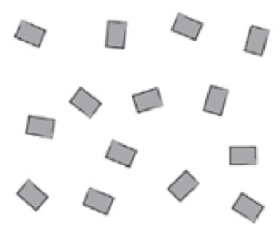

YYYYYYYY

Substrate to be grafted

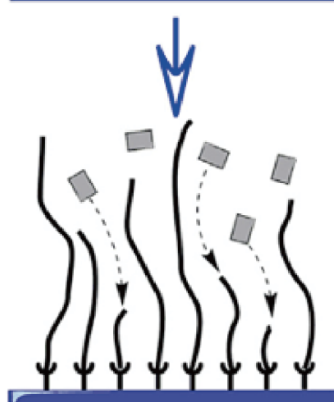

Substrate to be grafted

Figure 2.9 Schematic of the "grafting to", " grafting through", and "grafting from" approaches used for modification of particle surfaces. Circles: reactive groups to anchor on the surface or used as a chain-growth point (for the "grafting through" approach); lines: polymer chains; rectangles: monomer units (Image was adapted from reference 137).

In a classical surface-initiated ATRP reaction, alkyl halide initiators are anchored onto the NPs' surfaces first, followed by activation by the catalyst system (e.g., $\mathrm{Cu}$ //ligand complex) to form living radicals and a corresponding metal complex in a higher oxidation state (e.g., $\mathrm{Cu}^{\mathrm{II}} /$ ligand complex). ${ }^{128}$ During the chain propagation reaction, the radicals are deactivated reversibly by reacting with the $\mathrm{Cu}^{\mathrm{II}} /$ ligand complex. This reversible activation/deactivation process establishes an equilibrium with most of the growing chains in the dormant state during the polymerization. Thus, growing chains have an equal chance to add one or more monomer units during a short period of active growth time (at the rate of $\mathrm{kP}$ ), after which they return to the dormant state (see Figure 2.10). Compared to conventional radical polymerization, in which chains are grown for a very short time (typically $10^{-2} \mathrm{~s}$ to a few seconds), polymer chains from ATRP grow slowly 
and continuously throughout the polymerization period. Because the living radicals grow with equal probability throughout the entire polymerization process and termination is essentially suppressed, ATRP tends to have relatively low PDI values (1.05-1.2) compared to conventional free-radical polymerizations.

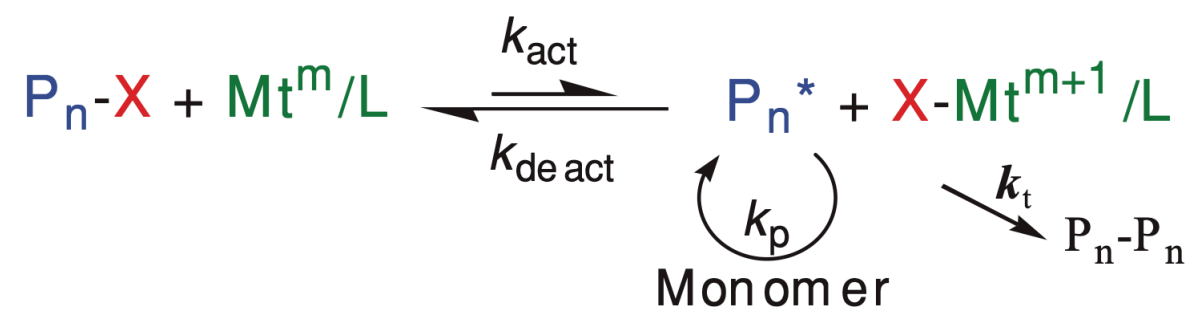

Figure 2.10 Schematic drawing of the ATRP equilibrium. ${ }^{138} P_{n}-X, P_{n}{ }^{*}, M t^{m} / L$, and $X$ $M t^{m+1} / L$ represent alkyl halides/macromolecular species, growing radicals, transition metal complexes in their lower oxidation state, and halide ligands coordinated transition metal complexes in their higher oxidation state, respectively. $k_{a c t}, k_{\text {deact, }}$ and $k_{t}$ refer to the activation, deactivation, and termination rate constants, respectively.

One of the first systematical studies utilizing surface-initiated ATRP with $\mathrm{SiO}_{2} \mathrm{NPs}$ was reported by Patten and von Werne. ${ }^{138,139}$ They demonstrated the fabrication of coreshell structured polymer- $\mathrm{SiO}_{2}$ NP hybrids with polystyrene and poly(methyl methacrylate) (PMMA). They also showed that the addition of sacrificial free initiators and deactivators $\left(\mathrm{Cu}^{\mathrm{II}}\right)$ provided a sufficient deactivator concentration which avoided uncontrolled polymer chain growth and particle aggregation. Nowadays, various polymers have been grafted from $\mathrm{SiO}_{2}$ via surface-initiated ATRP, including PS ${ }^{139,141}$, PMMA $^{139,140}$, poly(tert-butyl acrylate) (PtButA) ${ }^{140,141}$, and poly(oligo(ethylene glycol)methacrylate) (POEGMA) ${ }^{142}$. SiNPs typically form a surface-exposed oxide layer, when stored at ambient conditions, ${ }^{143}$ that can be readily modified with ATRP functional initiators by exploiting well-established silica-based chemical approaches. Thus, surfaceinitiated ATRP seems promising to fabricate stable dispersions of polymer-SiNP hybrids as electroconductive inks suitable for printing functional devices.

\subsubsection{Inkjet Printing of Silicon Nanoparticle Inks}

Unlike its bulk form, not much work has been done to utilize SiNPs in electronic devices, including their deposition by inkjet printing. To the best of our knowledge, most of the reports about inkjet printing of SiNP-based inks were published in the last decade. ${ }^{14-147}$ Gupta and coworkers are among the pioneers in utilizing inkjet printing for SiNP-based inks. ${ }^{147}$ In their work, gas-phase synthesized SiNPs were functionalized with ethyl undecylenate to form stable colloidal suspensions in various organic solvents. 
Moreover, they demonstrated using inkjet printing as a processing technique to print SiNP-based inks on glass substrates. ${ }^{147}$ Drahi et al. reported on the influence of SiNP surface chemistry and annealing procedure on the microstructure and thermal conductivity of inkjet-printed SiNP thin films. ${ }^{145}$ Furthermore, they demonstrated the successful utilization of photonic annealing for the sintering of inkjet-printed SiNP thin films. Compared to traditional thermal annealing, photonic annealing is faster and readily compatible with flexible and low-cost substrates. ${ }^{145}$ In a more recent paper, Lawes et al. present the fabrication of a lithium-ion battery anode via the inkjet printing of commercially available SiNPs. ${ }^{144}$ The SiNPs-based ink used in their study was formulated by mixing commercially available SiNPs, carbon black and polymer binders (poly(3,4-ethylenedioxythiophene)-poly(styrene sulfonate)

(PEDOT:PSS), polyvinylpyrrolidone (PVP), carboxymethyl cellulose (CMC), and Na-alginate) in water with extensive sonication. The lithium-ion battery prepared with an anode printed from the as-prepared SiNP-based inks showed a capacity of over $1700 \mathrm{~mA} \mathrm{~h} \mathrm{~g}^{-1}$ for 100 chargedischarge cycles. When the depth-of-discharge was limited to $1000 \mathrm{~mA} \mathrm{~h} \mathrm{~g}{ }^{-1}$, the prepared lithium-ion battery has a stable performance over 1000 cycles. The presented and discussed examples clearly demonstrate that the inkjet printing of SiNPs has a bright full future as key enabling technology in printable electronics.

\subsection{Silicon Nanoparticles as the Next Generation "Magic Bullets" in Biomedical Applications}

More than 100 years ago, Ehrlich postulated the concept of targeting drugs to an intended pathogen and treat it without affecting the surrounding healthy tissue. ${ }^{146}$ In popular terms, this is referred to as a magic bullet. ${ }^{149}$ It took until the early nineties to develop targeted drug delivery as a popular and well-established research field. Since then, the number of scientific reports has continued to increase. The targeting of a drug to specific sites in the body is of interest since it potentially allows for the administration of effective drugs to diseased cells or tissue without affecting the adjacent healthy cells and tissue and allows to reach hard-to-reach tissue/diseases. ${ }^{150}$ Eventually, this contributes to higher drug efficiency at potentially a less drug dose, with lower adverse side effects for the patient, lower costs, and a lower acquired immunity. As we will present and discuss in this section, SiNPs have an established track record in drug delivery applications. However, when looking beyond the field of solely targeted delivery of drugs, it it worth noted that due to the intrinsic properties of Si-containing NPs, they offer a promising platform as theranostic agents. In theranostics, a therapeutic component 
(e.g. a drug) is combined with (real-time) diagnostic functionalities. ${ }^{151-153}$ In the remainder of this section, we will present and discuss why SiNPs receive considerable attention as platform material in drug delivery and particularly in the emerging field of theranostics.

\subsubsection{Silicon Nanoparticles as Drug Delivery Carriers}

Since $\mathrm{Si}$ is known for its biocompatibility ${ }^{154-156}$ and is a naturally occurring element in various tissues in the human body (primarily present as orthosilicate (SiO44-)160), it is considered as a promising building unit for functional particles in biomedical applications. In addition, the degradation products of Si-containing NPs (mainly silicic acid) are non-toxic and readily renal clearable. ${ }^{155,157,158}$ These properties render Sicontaining NPs an interesting alternative to compete with NP-based systems for which toxicological and biodegradability concerns exist, like gold NPs, carbon NPs, and various quantum dots. ${ }^{159}$

Besides being biocompatible and biodegradable, a targeted drug carrier must meet the following additional criteria to be effective for: i) high drug loading, ii) preservation of drug functionality, and iii) effective targeting. Among the various NP-based platforms, porous SiNPs have attracted considerable attention since they meet these criteria. ${ }^{160-163}$ Therefore, in biomedical nanotechnology, SiNPs are abundantly reported as drug carriers. Since it is not the scope of this work to review the use of SiNPs as drug carriers in-depth, the reader is directed to some selected review papers that cover this in-depth and are considered to be of interest. ${ }^{164-168}$

Porous SiNPs are produced mainly by the electrochemical anodization of monocrystalline Si wafers. $5,13,164,169,170$ This method uses hydrofluoric acid as the electrolyte in various organic solvents, such as ethanol or acetonitrile. The produced porous Si film is lifted from the Si wafer, and following sonication or milling treatments, micro and NPs are formed. This results in the formation of quasi-spherical porous SiNPs with a relatively broad particle size distribution. 


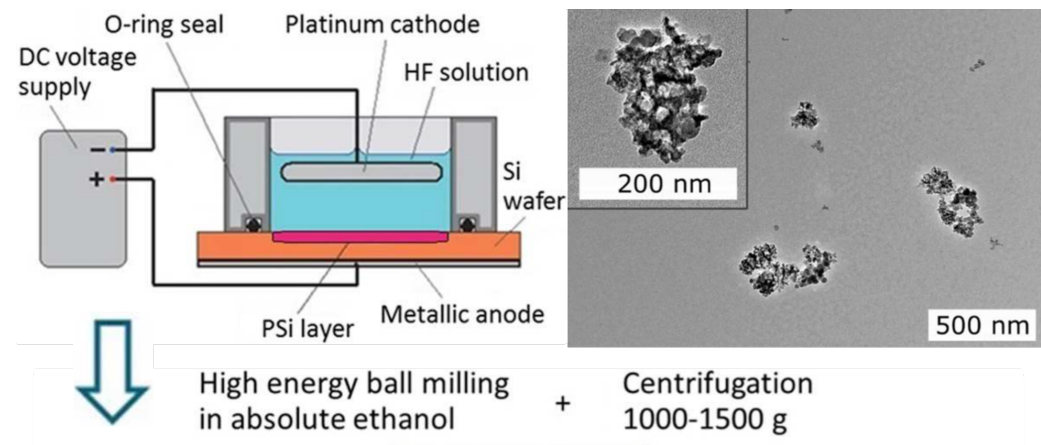

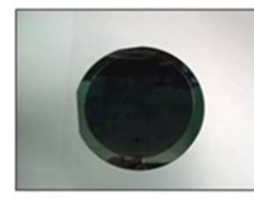

Porous silicon layer

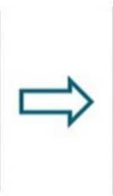

PSi powder

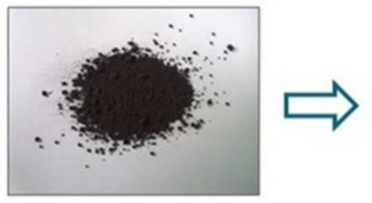

Suspension of PSi nanoparticles

Figure 2.11 Scheme for the preparation of SiNPs by electrochemical etching and TEM image of as-prepared SiNPs. (Picture was modified.) ${ }^{170}$

The high porosity (up to $80 \%$ ) of SiNPs then allows one to obtain high drug loadings. ${ }^{171}$ It is of pivotal importance to tailor the physicochemical properties of SiNPs to control their degradation time in the bloodstream/targeted tissues. ${ }^{162,175-177}$ It is wellknown that the pore size and porosity significantly affect the degradation rate of SiNPs under physiological conditions. ${ }^{159}$ In addition, without altering the surface chemical composition, SiNPs tend to degrade relatively quickly. The surface of porous SiNPs mainly consists of silanols, siloxanes, and silicon hydrides. These surface-exposed moieties are readily used in well-known surface chemical engineering processes ${ }^{160}$ to tailor surface chemistry and enhance affinity towards specific drugs (hydrophobic or hydrophilic) allowing controlled incorporation and release . In fact, carefully selecting the chemical composition allows one to prolong the SiNPs' lifetime and incorporate a gating function that can control the release of the intended drugs. ${ }^{160}$ In addition, tuning the SiNPs surface chemistry facilitates a better dispersion in aqueous media as well as that targeting often required in vivo stealth functionalization. ${ }^{161,164,172-174}$

For instance, to enhance the blood circulation time of SiNPs, Santos and coworkers ${ }^{175}$ reported red blood cell membrane coated porous SiNPs that were loaded with radioactive $155 \mathrm{~Tb}$ atoms. The red blood cell membrane coating significantly enhanced the blood half-life compared to bare Si NPs. The authors concluded that the prepared $155 \mathrm{~Tb}$ loaded SiNPs could be used in targeted radionuclide therapy combined with a chemotherapeutic payload within the porous SiNPs. In another report, Santos and coworkers ${ }^{176}$ showed that 
the covalent attachment of a model cancer antigen TRP2, either expressed at the membrane surface or covered by membrane encapsulation, is promising for treating autoimmune diseases by inducing an autoantigen specific immune tolerance. Weitz and coworkers ${ }^{177}$ designed and prepared hybrid polymersomes as a versatile drug delivery system. Their design was based on utilizing porous SiNPs conjugated with gold nanorods encapsulated within a hybrid polymersome by using double emulsion templates on a microfluidic chip. This particle design allowed the incorporation of hydrophilic and hydrophobic drugs, and it was demonstrated that the in vivo drug delivery efficiency to tumors in mice improved. An exciting feature of this hybrid system is that the conjugated gold nanorods absorb near-infrared laser radiation, which triggered the increased release of doxorubicin hydrochloride and rapamycin under near-infrared laser irradiation during in vitro tests. This feature suggests that this hybrid polymer-based drug delivery system has the potential to be applied in photothermal therapy.

Besides the relatively easy tunability of particle geometry and surface chemistry of Si-containing NPs to control the SiNPs drug release rate and biodegradability, they have interesting optoelectronic properties that allow the incorporation of multiple functionalities in one particle. ${ }^{177-180}$ This makes that SiNPs receive considerable attention during the last decade as a multimodal platform for theranostic applications. ${ }^{180}$ The following sections (2.4.2 and 2.4.3) will introduce the main concepts of SiNP based (enhanced) imaging in combination with local disease treatment.

\subsubsection{Photoluminescent Silicon Nanoclusters and Bioimaging}

The earlier discussed biocompatibility and unique optical properties make PL SiNPs a novel, high-quality, and biocompatible fluorescent probe for in-vivo imaging. Of particular interest is that SiNPs can be used in near-infrared laser two-photon-excited fluorescence imaging. ${ }^{181-184}$ This allows deeper tissue penetration, and it provides a better spatial selectivity with a reduced autofluorescence of the surrounding biological tissues. $^{185,186}$

Numerous synthetic strategies - physical, physiochemical, chemical, and electrochemical - have been proposed and developed to fabricate PL SiNPs with a high quantum yield and long-term photostability. ${ }^{6,187-190}$ For example, Zhong et al. ${ }^{191}$ reported a method to synthesize PL SiNPs with microwave irradiation. Using organosilicon molecules as precursors, they produced $0.1 \mathrm{~g}$ PL SiNPs with a quantum yield of $20-25 \%$ in less than 10 minutes. In addition, Zhong et al. ${ }^{192}$ later reported the fabrication of PL SiNPs by UV radiation of (3-aminopropyl)trimethoxysilane and 1,8-naphthalimide under ambient conditions with a high production rate (i.e. $10 \mathrm{~g}$ of PL SiNPs in $40 \mathrm{mins}$ ). ${ }^{191,192}$ 

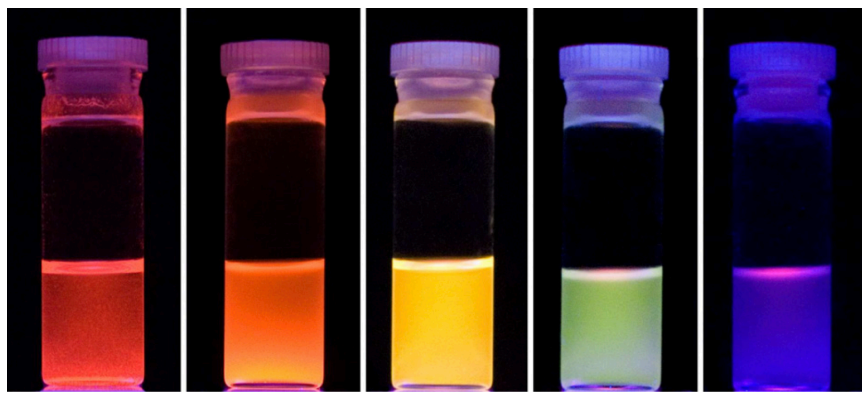

Figure 2.12 Photographs of deep red $(740 \mathrm{~nm})$, red $(675 \mathrm{~nm})$, yellow $(592 \mathrm{~nm})$, green $(572 \mathrm{~nm})$, and blue $(441 \mathrm{~nm})$ light emission from Si NCs when excited at $365 \mathrm{~nm} .{ }^{193}$

Besides being able to tune the (optical) properties of SiNPs towards specific in vivo applications, their interactions with living cells must be thoroughly understood as well. Of particular importance is that the foreign SiNPs do not induce any toxic effects on the surrounding tissue. Therefore, the cellular behavior of PL SiNPs has been investigated and monitored in living cells by various researchers. ${ }^{194-197}$ For instance, Cao et al. ${ }^{194}$ described the detailed mechanism of SiNPs entering living cells. SiNPs were shown to be internalized into cells via clathrin-mediated and caveolae-dependent endocytosis. These authors also demonstrated that SiNPs showed no apparent toxic effects on cell metabolic activity. Recently, Zhou et al. ${ }^{197}$ studied the in-vivo behavior and molecular imaging of PL SiNPs inside Caenorhabditis elegans. They demonstrated that PL SiNPs could be used as nanoprobes for bioimaging inside living organisms.

Various biocompatible molecules like sugar, transferrin, targeting peptides, and polyethyleneimine (PEI), have been used to decorate PL SiNP surfaces for cancer targeting, ${ }^{195,196}$ molecular trackings, ${ }^{197}$ and monitoring carbohydrate-carbohydrate interaction dynamics ${ }^{198}$. Pang et al. ${ }^{199}$ developed PEI-encapsulated SiNPs (PEI-SiNPs) to observe the behavior of gene carriers in living cells in real-time. Their long-term stable PEI-SiNPs showed a DNA-loading capacity of $97 \%$ and an adaptable efficiency of $35 \%$ for human cervical carcinoma cells, with the capability to maintain $90 \%$ of cell viability for 24 and 48 hours after treatment. By attaching peptides (cyclic arginine-glycineaspartic acid (RGD)) on the PL SiNPs surface, they demonstrated the use of SiNP-RGD as a suitable bio-probe to image cancer cells in real-time. These SiNP-RGDs also showed highly efficient anticancer properties.

Recently, He and coworkers ${ }^{200}$ studied the use of vancomycin (i.e. a glycopeptide antibiotic) modified SiNPs as a theranostic component in eye drops to diagnose and treat Gram-positive bacteria-induced keratitis (an eye infection). They successfully exploited the high binding affinity of vancomycin to dipeptides on the cell wall of Gram-positive 
bacteria to target the incorporated fluorescent SiNPs and by optically monitoring the fluorescence to diagnose keratitis within 10 minutes. In addition, the antimicrobial rate for vancomycin immobilized on SiNPs is $92.5 \%$, which is nearly four times higher than free vancomycin at the same concentration. Therefore, the vancomycin-modified SiNPs are considered an excellent nano theranostics agent capable of simultaneously diagnosing and treating bacteria keratitis.

Interestingly, SiNPs can act as photosensitizers in photodynamic therapy as well. ${ }^{201-}$ ${ }^{204}$ For instance, the in vitro PL visualization of SiNPs in living cells and the production of singlet oxygen in these cells was successfully demonstrated by Timoshenko and coworkers. ${ }^{203}$ Singlet oxygen is proven to be effective in the treatment of cancer. ${ }^{205}$ Furthermore, SiNPs are exploited in photothermal therapy in which near-infrared irradiation is used to generate heat locally, resulting in targeted cell death without harming the surrounding tissue. ${ }^{206,207}$ Besides using near-infrared in photodynamic therapy, thermotherapy with SiNPs is possible with ultrasound ${ }^{169}$ and radiofrequency ${ }^{208,209}$ radiation.

\subsubsection{Silicon Nanoparticle and Magnetic Resonance Imaging}

Magnetic resonance imaging (MRI) is a non-invasive technique that is used, among others, for in-vivo studies and clinical diagnostics. However, the main challenge of MRI in molecular imaging is the inherently low sensitivity, especially when detecting nuclei with low gyromagnetic ratios and low natural abundancies like ${ }^{13} \mathrm{C},{ }^{29} \mathrm{Si}$, and ${ }^{15} \mathrm{~N}$. Hyperpolarization is a technique that temporarily increases the nuclear spin polarization of a material far beyond its thermal equilibrium, which enhances the detectability and shortens the MRI acquisition time for nuclei with low gyromagnetic ratios (see Figure 2.13). ${ }^{210,211}$ However, small hyperpolarized ${ }^{13} \mathrm{C}$-labeled substrates like $\left[1-{ }^{13} \mathrm{C}\right]$ pyruvic often rapidly relax to their equilibrium states (i.e. within $120 \mathrm{~s}$ ), making it hard to monitor slow biological processes like protein-cell binding and tissue perfusion. ${ }^{212,213} \mathrm{Si}$ in the form of particles has a nuclear spin relaxation $\left(T_{1}\right)$ time up to several hours and coherence $\left(\mathrm{T}_{2}\right)$ times up to several seconds at room temperature. ${ }^{214,215}$ The long $\mathrm{T}_{1}$ for $\mathrm{Si}$ is due to the existence of a small amount of spin $-1 / 2{ }^{29} \mathrm{Si}$ nuclei in a nuclei-spin-free lattice. The extraordinary long $\mathrm{T}_{1}$ and the already mentioned outstanding biocompatibility make SiNPs a promising imaging agent for in-vivo hyperpolarized ${ }^{29} \mathrm{Si}$ MRI. 


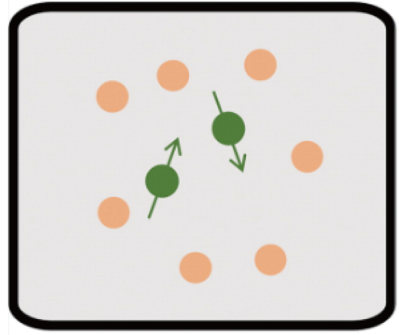

Normal Equilibrium

- Very few carbon atoms are ${ }^{13} \mathrm{C}$-labeled

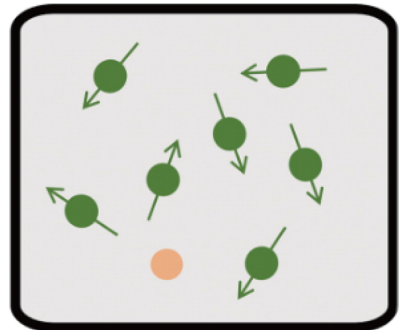

${ }^{13} \mathrm{C}$ Enrichment

- Increased number of

${ }^{13} \mathrm{C}$-labeled carbon atoms

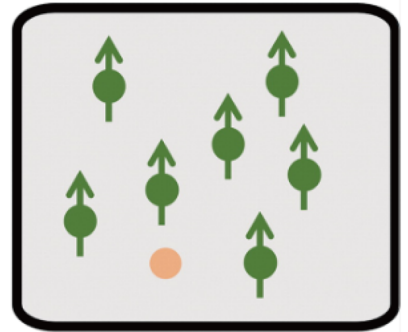

Hyperpolarization

- DNP increases the number of aligned spins

- Spins are not well-aligned

Figure 2.13 Increase of MRI signal intensity of ${ }^{13} \mathrm{C}$ nuclei via hyperpolarization. DNP = dynamic nuclear polarization, $N M R=$ nuclear magnetic resonance. ${ }^{216}$

The first demonstration of using hyperpolarized Si particles with diameters from 100 $\mathrm{nm}$ to $1 \mu \mathrm{m}$ as MRI agents was reported by Aptekar et al. ${ }^{217}$ The room temperature $\mathrm{T}_{1}$ for these particles ranged from tens of minutes up to hours depending on the particle size and doping. Inspired by their results, chemists and material scientists developed various synthetic procedures to fabricate $\mathrm{Si}$ particles with enhanced $\mathrm{T}_{1}$ values that could potentially monitor the biochemical reaction in-vivo. For example, Tonya et al. ${ }^{218}$ used a metathesis reaction of sodium silicide with silicon tetrachloride to fabricate $10 \mathrm{~nm}$ SiNPs, followed by surface modification with alkyl and aromatic ligands. The resulting particles showed $\mathrm{T}_{1}$ times greater than $600 \mathrm{~s}$, which is five times longer than conventional ballmilled (and larger) SiNPs. Their small particle size allows them to cross the blood-brain barrier and enables their utilization in intracellular studies with MRI.

Whiting et al. ${ }^{219}$ exploited relatively low-cost commercial Si particles with a diameter of $2 \mu \mathrm{m}$ to demonstrate the use of hyperpolarized ${ }^{29} \mathrm{Si} \mathrm{MRI}$ for in-vivo, real-time passive angiocatheter tracking in two and three dimensions for over 40 minutes. Advantages of using MRI guidance compared to currently used X-ray fluoroscopy are that MRI allows three-dimensional imaging, other physiologically relevant criteria can be monitored, and the clinical team is not exposed to ionizing radiation. Though promising to replace X-ray fluoroscopy guidance, the proof of concept MRI-based guidance suffered from a lack of spatial resolution $(\sim 1 \mathrm{~mm}$ for MRI guidance versus $\sim 0.1 \mathrm{~mm}$ for X-ray fluoroscopy). According to the research team, moderate improvements to the ${ }^{29} \mathrm{Si}$ hyperpolarization level, MR hardware, and pulse sequences are required to overcome this limitation. Interestingly, in a follow-up study Whiting and coworkers ${ }^{220}$ demonstrated that the 
functionalization of commercial SiNPs with APTES, PEG, and oligonucleotide aptamer had little effect on the hyperpolarization buildup, or decay rates, for these particles.

Overall, we can conclude that SiNPs are an exciting platform for multimodal imaging and/or therapeutic applications. Although the research field of theranostic is relatively young, it receives considerable attention, and it is believed that due to their unique properties in combination with good biocompatibility, SiNPs will play an essential role in this regard. Future directions to consider are the development of scalable and reproducible production methods of SiNPs at acceptable costs and to get regulatory clearance for these functional particles. ${ }^{221}$

\subsection{Surface-Engineered Silica Nanoparticles}

$\mathrm{SiO}_{2} \mathrm{NPs}$ are another class of NPs that cannot be neglected when considering Sicontaining NPs. Unlike SiNPs, $\mathrm{SiO}_{2}$ NPs typically do not possess new properties compared to their bulk form. However, $\mathrm{SiO}_{2} \mathrm{NPs}$ have the advantage of being cheap and available with a controlled and narrow size distribution. Furthermore, $\mathrm{SiO}_{2} \mathrm{NPs}$ provide ample opportunities to adapt their morphology and are readily derivated by widely accessible surface functionalization strategies. ${ }^{222}$ Like other $\mathrm{NPs}, \mathrm{SiO}_{2} \mathrm{NPs}$ also have a large surface-to-volume ratio, which provides extremely large interface areas upon adding only a few wt $\%$ of particles to polymer composites. ${ }^{223-225}$ While most of the $\mathrm{SiO}_{2}$ NP-based thermoplastic composites rely on the use of rather smooth particles, the introduction of particle surface roughness allows one to tailor and eventually enhance the surface/interface properties of the resulting composites. This section introduces common strategies to improve $\mathrm{SiO}_{2} \mathrm{NP}$ surface roughness and subsequently we present and discuss how this can significantly enhance polymer foam cell nucleation. We recently introduced the latter as an emerging application area of surface engineered silica NPs. ${ }^{225}$

\subsubsection{Surface Roughness Engineering of Silica Nanoparticles}

Surface roughened $\mathrm{SiO}_{2} \mathrm{NP}\left(\mathrm{SiO}_{2}-\mathrm{R} \mathrm{NP}\right)$ based layers are considered an effective coating material to mimic the structure of lotus leaves. ${ }^{226-228}$ The nanocavities that reside on the surface of the assembled NPs reduce the contact area between a droplet and the surface to minimize the adhesion, resulting in superhydrophobic surface behaviour. ${ }^{229,230}$ 
a.

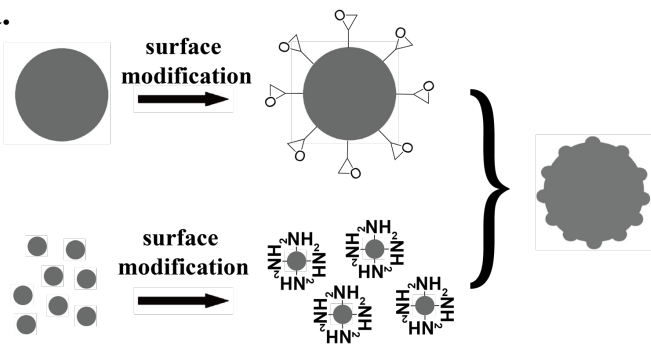

b.

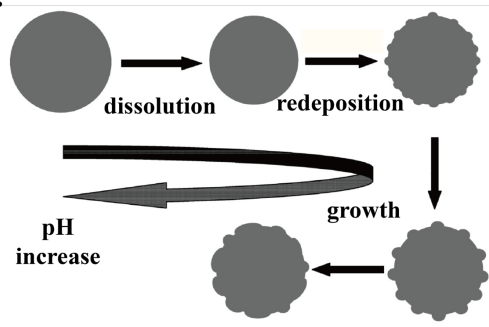

Figure 2.14 Schematic of a) the attachment approach ${ }^{227}$ and b) the etching approach ${ }^{97}$ for the fabricating of $\mathrm{SiO}_{2}-\mathrm{R} \mathrm{NPS}$.

Chemists and materials scientists have developed different synthetic strategies to prepare $\mathrm{SiO}_{2}-\mathrm{R} \mathrm{NP}$. The most common approaches to synthesizing $\mathrm{SiO}_{2}-\mathrm{R} \mathrm{NP}$ are the attachment and etching methods (see Figure 2.14). ${ }^{226,227,231-234}$ The attachment method is usually a two-step reaction to attach smaller NPs to larger core particles, governed by various interactions, such as complexation, covalent bonding, and hydrogen bonding. For instance, $\mathrm{Hu}$ et al. ${ }^{231}$ reported the fabrication of stimuli-responsive raspberry-like hollow mesoporous colloids by utilizing host-guest complexation. The separately prepared viologen functionalized $\mathrm{Fe}_{3} \mathrm{O}_{4} \mathrm{NPs}$ that were subsequently attached to azobenzene functionalized $\mathrm{SiO}_{2}$ core particles via the host-guest complexation of cucurbituril molecules. Upon UV-irradiation, the $\mathrm{SiO}_{2}$ core particles can grab or release the $\mathrm{Fe}_{3} \mathrm{O}_{4}$ NPs. Alternatively, Blanckenberg et al. ${ }^{234}$ synthesized raspberry-like gold-decorated $\mathrm{SiO}_{2}$ NPs $\left(\mathrm{SS}_{\mathrm{x}}\right.$-AMPS- $\left.\mathrm{Au}_{\mathrm{n}}\right)$ by growing the secondary particles from the primary particle surface. A gold(III) chloride trihydrate $\left(\mathrm{HAuCl}_{4}\right)$ solution was added to an aminofunctionalized $\mathrm{SiO}_{2} \mathrm{NP}$ suspension to grow gold NPs on the silica surface. The particles were then used to catalyze the sodium borohydride reductive discoloration of methylene blue in the water. Ming et al. ${ }^{227}$ introduced the covalent bonding between two different sizes of $\mathrm{SiO}_{2}$ using either amino-functionalization or epoxy-functionalization to yield $\mathrm{SiO}_{2}-\mathrm{R}$ NPs. Subsequently, the $\mathrm{SiO}_{2}-\mathrm{R}$ NPs were deposited on an epoxy film, followed by coating the deposited particles with a PDMS layer to form a dual-sized structured superhydrophobic surface. Instead of coupling amino and epoxy groups to fabricate $\mathrm{SiO}_{2}$ R NPs, Puretskiy et al. ${ }^{226}$ exploited poly(glycidyl methacrylate) polymer brushes as the coupling agent to achieve better mechanical stability and chemical resistance of $\mathrm{SiO}_{2}-\mathrm{R}$ NP (see Figure 2.15). They also reported poly(pentafluorostyrene) immobilization on the as-prepared $\mathrm{SiO}_{2}-\mathrm{R} \mathrm{NPs}$ to obtain a superhydrophobic surface. The major advantage of the attachment approach is that $\mathrm{SiO}_{2}-\mathrm{R}$ NPs with a controlled surface roughness can be fabricated by simply adjusting the diameter and concentration ratio of large and small particles. However, the attaching approach typically requires multiple reaction steps. In addition, because the large and small particles are connected by large molecules or 
polymer chains, small particles can be detached from the large particle under extreme conditions, like long-term sonication or extrusion.

Another approach encompasses the etching method, which uses the dissolution and re-deposition (growth) of $\mathrm{SiO}_{2}$ to fabricate $\mathrm{SiO}_{2}-\mathrm{R}$ NP surfaces. For example, Du et al. ${ }^{97}$ synthesized $\mathrm{SiO}_{2}-\mathrm{R}$ particles by etching nearly spherical $\mathrm{SiO}_{2} \mathrm{NPs}$ with $\mathrm{NaBH}_{4}$. The dissociation and re-deposition of Si-O bonds on the surface of $\mathrm{SiO}_{2} \mathrm{NPs}_{\mathrm{s}}$ at high $\mathrm{pH}$ results in the formation of $\mathrm{SiO}_{2}-\mathrm{R}$. Those particles were deposited on polycation and polyanioncoated glass substrates. The particle deposition step was followed by calcination to remove the hydrophilic -OH group from the particle surface. Finally, the as-prepared glass substrates were coated with $1 \mathrm{H}, 1 \mathrm{H}, 2 \mathrm{H}-$, and $2 \mathrm{H}$-perfluorooctyltriethoxysilane to form superhydrophobic surfaces. Due to the nature of the etching process, the surface roughness of the $\mathrm{SiO}_{2}-\mathrm{R}$ is somewhat unpredictable, and according to our experience, it may vary from batch to batch, hampering its wide spread applicability.
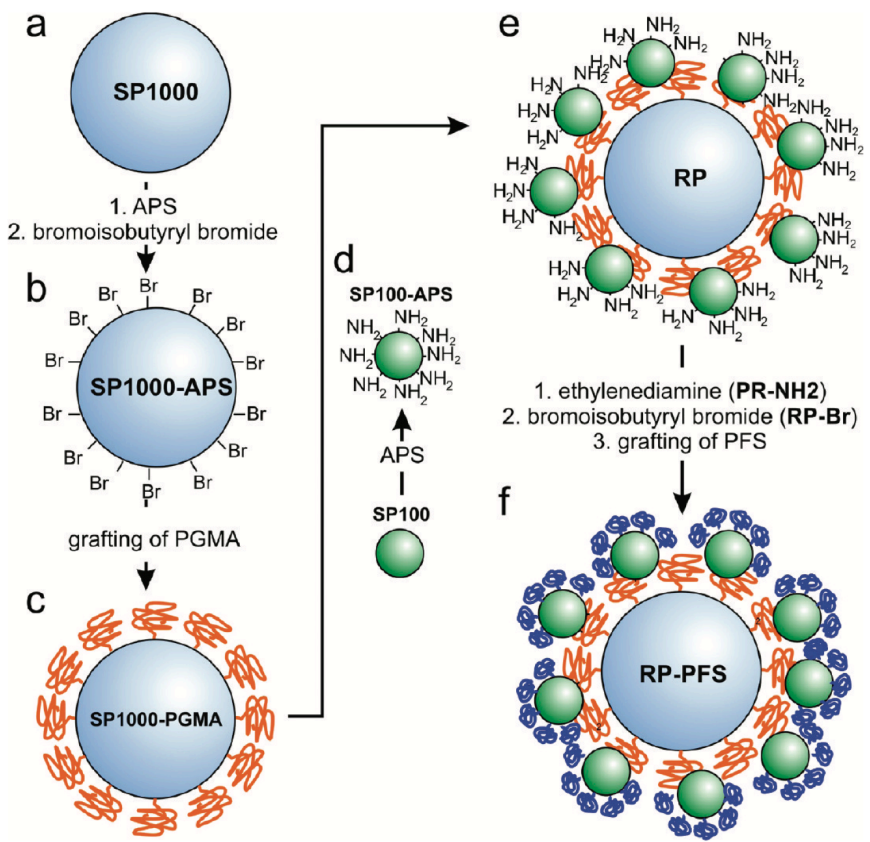

Figure 2.15 Schematic of $\mathrm{SiO}_{2}-\mathrm{R} N \mathrm{~N}$ synthesis. a-c) large particles were grafted with poly(glycidyl methacrylate); d) Small particles are modified with APTES; e) Coupling of large and small particles; f) Immobilizing polymer onto the $\mathrm{SiO}_{2}-\mathrm{R}$ surface. ${ }^{226}$

Alternatively to the etching and attaching method, Chen et al. ${ }^{235}$ recently reported a one-step dialysis strategy to fabricate $\mathrm{SiO}_{2}-\mathrm{R}$ NPs at mild processing conditions. First, a homogeneous mixture of TEOS, $\mathrm{H}_{2} \mathrm{O}$, and ethanol was added to a dialysis bag. This step 
is followed by immersing the dialysis bag into a large reservoir filled with an ammonia/water mixture (10\% volume ammonia) to react for four hours. The resulting temporal ammonia concentration gradient results in the formation of porous raspberrylike $\mathrm{SiO}_{2} \mathrm{NP}$ formation. This method is considered to be of particular interest since it does not use any other chemicals than typically used to synthesize $\mathrm{SiO}_{2}$ NPs.

Most of the literature on surface roughness modified $\mathrm{SiO}_{2} \mathrm{NP}$ is focused on utilizing $\mathrm{SiO}_{2}-\mathrm{R}$ NPs to create dual-sized structures to induce superhydrophobicity or using $\mathrm{SiO}_{2}$ NP as a support for other functional particles. However, by carefully examining the morphology of $\mathrm{SiO}_{2}-\mathrm{R}$ NPs and thoroughly investigating the heterogeneous foam cell nucleation theory, we have successfully designed and utilized $\mathrm{SiO}_{2}-\mathrm{R}$ as a highly efficient nucleating agent for polymer foam cell nucleation. This is presented and discussed in the following section.

\subsubsection{Foam Cell Nucleation by Surface Topology Engineered Silica Nanoparticles}

Nucleation is a process of creating a new thermodynamic phase with lower free energy incorporated within a metastable phase with a relatively high free energy. Nucleation can happen in the gas, liquid, and solid phases, e.g., rain, boiling, crystallization. In this part, we focus on gas-liquid nucleation.

Gas-liquid nucleation processes can be either homogeneous or heterogeneous. For homogeneous nucleation, the free energy for critical nucleus formation is ${ }^{236}$

$$
\Delta G_{\text {crit }}=\frac{16 \pi \sigma^{3}}{3 \Delta P^{2}}
$$

in which $\Delta P$ is the pressure difference between two phases if assuming the partial molar volume of gas (e.g. $\mathrm{CO}_{2}$ ) in the polymer after full saturation is $0 . \sigma$ is the interfacial tension between the metastable phase and nucleating phase. However, since $\sigma$ is experimentally inaccessible, it is usually replaced by the surface tension of a flat surface on the macro scale. 


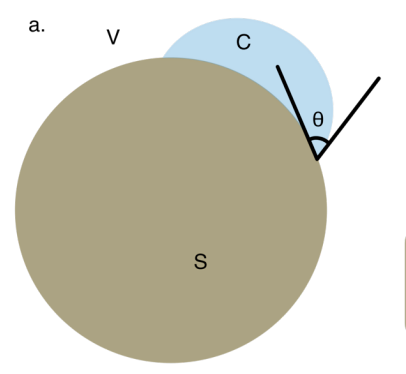

b.

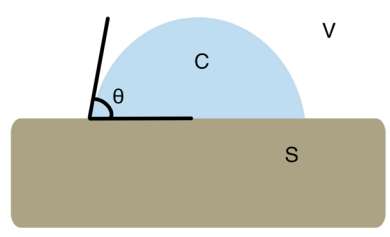

c.

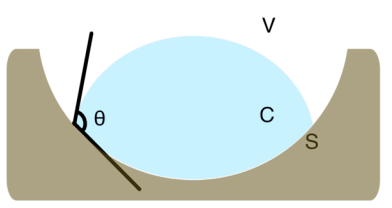

Figure 2.16 Schematic illustration of nucleation a) on the spherical particle surface, $b$ ) on a flat surface, and c) inside a cavity.

Heterogeneous nucleation is considered thermodynamically more favorable than homogeneous nucleation when the contact angle $(\theta)$, as defined in Figure 2.16, is smaller than $180^{\circ} .{ }^{237}$ A relatively simple heterogeneous nucleation model was deduced and reported by Volmer in 1929.238 This model assumes that liquid clusters grow on a planar substrate as a spherical cap with a constant wetting angle. Fletcher took this model a step further and extended Volmer's model to gas nucleation on a spherical particle surface. ${ }^{239}$ In his work, the particle curvature plays a vital role in the nucleation energy:

$$
\Delta G_{c r i t}^{h e t}=\frac{16 \pi \sigma^{3}}{3 \Delta P^{2}} * f(m, x)
$$

where $f(m, x)$ is the energy reduction factor, which depends on the curvature (x) and contact angle $(\theta)$. For spherical particles (i.e., a convex substrate, see Figure 2.16a), it is described as

$$
f(m, x)=\frac{1}{2}+\frac{1}{2}\left(\frac{1-m x}{g}\right)^{3}+\frac{1}{2} x^{3}\left[2-3\left(\frac{x-m}{g}\right)+\left(\frac{x-m}{g}\right)^{3}\right]+\frac{3}{2} m x^{2}\left(\frac{x-m}{g}-1\right)
$$

in which $\mathrm{m}$, w, and $\mathrm{g}$ are

$$
\begin{gathered}
m=\cos \theta \\
x=\frac{R}{r_{\text {crit }}} \\
g=\sqrt{1+x^{2}-2 m x} \\
r=\frac{2 \sigma}{P_{G}-P_{L}} .
\end{gathered}
$$

The extension of Fletcher's work to nanocavities (i.e. a concave surface, see Figure 2.16c) was reported by Liu et al. ${ }^{240}$ By assuming that the nanocavity is a smooth hollow 
sphere, they deduced that the influence factor $\mathrm{f}(\mathrm{m}, \mathrm{x})$ for a nanocavity is described a

$$
f(m, x)=1+\left(\frac{1+m x}{g}\right)+x^{3}\left[2-3\left(\frac{x+m}{g}\right)+\left(\frac{x+m}{g}\right)^{3}\right]+3 m w^{2}\left(\frac{x+m}{g}-1\right) \quad \text { eq. } 8
$$

in which $\mathrm{g}$ is

$$
\mathrm{g}=\sqrt{1+x^{2}+2 m x} .
$$

According to the results reported by Liu et al., ${ }^{240}$ nucleation is energetically favorable inside the cavities compared to nucleation by convex and flat surfaces of the same material. ${ }^{241} \mathrm{~A}$ schematic illustration of a liquid bubble nucleating on the particle surface and inside nanocavity is shown in Figure 2.16. Based on their results for nucleation inside cavities, $\mathrm{SiO}_{2}-\mathrm{R}$ was considered a potentially efficient heterogeneous foam cell nucleation agent in nanocellular thermoplastic foaming.

\subsubsection{Polymer Nanocomposite Foams}

Polymer foaming is a high-profile research area. Low density, nanocellular polymer nanocomposite foams are promising candidates for several applications, especially highperformance thermal insulation. ${ }^{241,242}$ Nowadays, polymer foams can be fabricated either by a continuous process, like extrusion foaming, or a non-continuous process, like batch foaming or injection molding foaming. ${ }^{243}$ Continuous foaming typically requires high temperature, high pressure, and high shear rates. Despite this, continuous foaming is widely accepted and applied in the industry since it is a quick, versatile and straightforward process. ${ }^{244}$ Compared to continuous foaming, non-continuous foaming is a more commonly used approach to prepare nanocellular polymer foams in the lab. In a typical batch foaming process, a polymer sample is saturated with a blowing agent like $\mathrm{CO}_{2}$ at a specific temperature and pressure. This is followed by releasing the pressure and increasing the temperature to oversaturate the dissolved blowing agent in the polymer matrix, which will induce foam cell nucleation and result in polymer foaming (see Figure 2.17). The major downside of batch foaming is the often required long saturation time (up to weeks), making this method less desirable to be applied at an industrial scale compared to continuous foaming. ${ }^{244}$ However, due to the easily adjustable and precise process control for each step during batch foaming and the good foam reproducibility, batch foaming is considered a powerful tool to investigate the foaming behavior of polymers. 


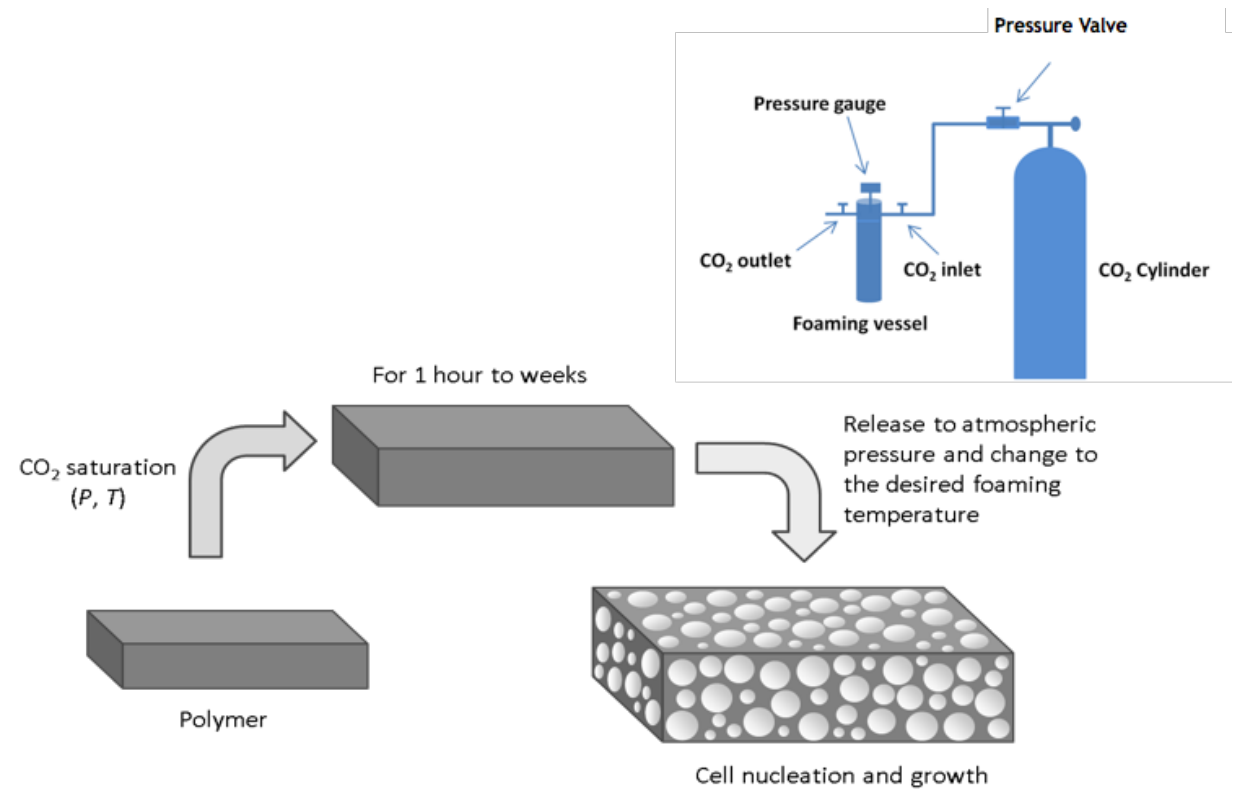

Figure 2.17 Schematic diagram of batch foaming process. ${ }^{242}$

Nanofillers are extensively discussed as heterogeneous nucleation agents for polymer foaming. ${ }^{245}$ Typically, the addition of nanofillers can dramatically increase the cell density of polymer foams (see Figure 2.18 ) by rendering heterogeneous nucleation favorable over homogenous nucleation. ${ }^{246}$ Colton and Suh were the first to demonstrate the use of nanofillers in polymer foaming. ${ }^{247-249}$ They reported that a high cell density was achieved by adding zinc stearate as the heterogeneous nucleation agent in polystyrene (PS) foaming. ${ }^{250-252}$ In addition to increasing cell density, introducing welldispersed nanofillers to polymers prior to foaming also produces foam cells with narrower size distributions. For example, by adding $9 \mathrm{wt} \%$ of nano-silica to PS, the foaming density increased from $10^{11}$ to $10^{13}$ cells $\mathrm{cm}^{-1}$, and the cell size was reduced from 0.7-6.0 $\mu \mathrm{m}$ to $0.1-0.8 \mu \mathrm{m}$ compared to neat PS foams. ${ }^{253}$ Various polymer and nanofiller combinations have shown that the addition of nanofillers results in increasing nucleation efficiency as well as in increasing the cell density, e.g., low-density polyethylene (LDPE)-clay ${ }^{254}$, PS-carbon nanofiber $(\mathrm{CNF})^{255}$, PS-clay ${ }^{256}, \mathrm{PS}^{-\mathrm{SiO}_{2}} \mathrm{NP}^{257}$, polymethyl methacrylate (PMMA)-clay ${ }^{258}$, PMMA-multi-walled carbon nanotubes (MWCNTs) ${ }^{258}$, $\mathrm{PMMA}_{-} \mathrm{SiO}_{2} \mathrm{NP}^{259}$, styrene acrylonitrile resin (SAN)-clay ${ }^{260}$, and polyurethane (PU)$\operatorname{clay}^{261}$. 


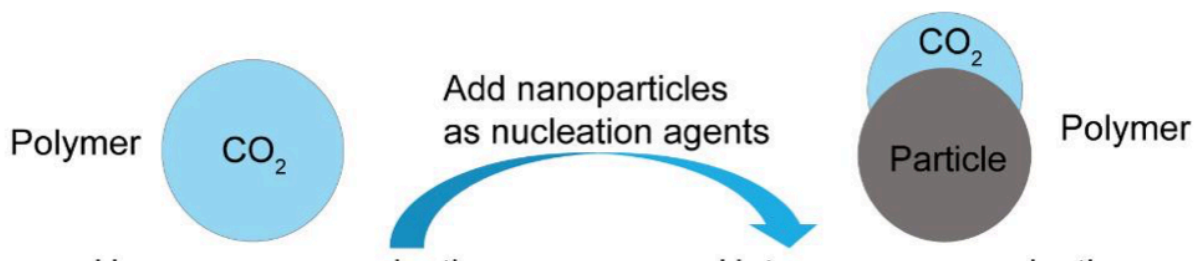

Homogeneous nucleation

Heterogeneous nucleation
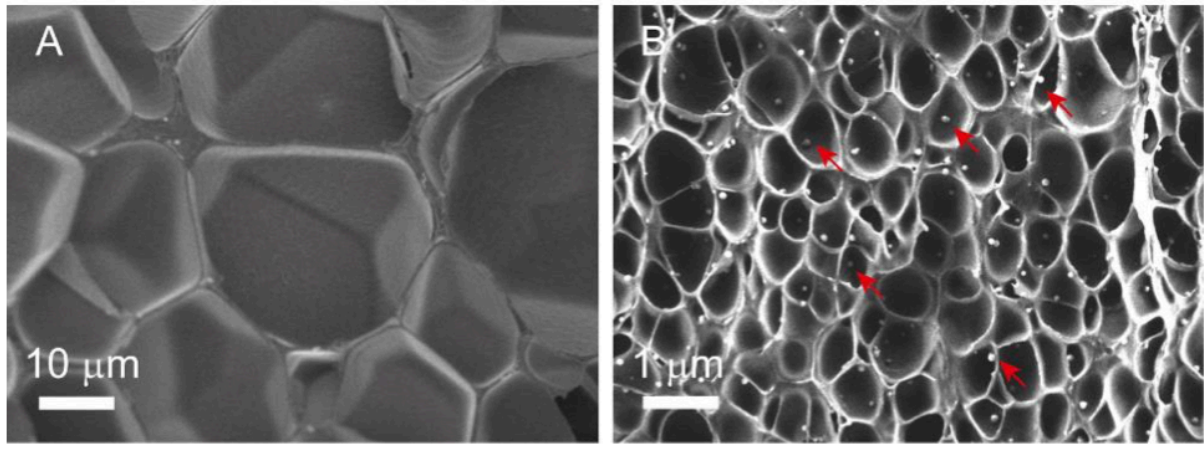

Figure 2.18 SEM images of the cross-section of polystyrene foams batch foamed without (A) and with silica-based hybrid NPs as heterogeneous nucleation agents (B). The red arrows in (B) point at some hybrid $\mathrm{SiO}_{2} \mathrm{NPs}^{262}$

In the last decade, Vancso and coworkers ${ }^{262-266}$ reported developing a series of silica core-shell NPs with different polymer shells to enhance the $\mathrm{SiO}_{2}$ particle dispersion in the polymer matrix and increase the foam cell nucleation efficiency. With PDMS-grafted silica core-shell NPs ( $\mathrm{SiO}_{2}$-PDMS) dispersed in PS film at $4.0 \mathrm{wt} \%$, the cell size and cell density of batched foamed nanocomposites were $600 \mathrm{~nm}$ and $4.6 \times 10^{12}$ cells $\mathrm{cm}^{-1}$, respectively. The nucleation efficiency is approximately $0.33 .{ }^{264}$ For PMMA SiO $2-\mathrm{PDMS}$ nanocomposites foams, the highest nucleation efficiency achieved is approximately 0.5. ${ }^{262}$ So far, this is the highest nucleation efficiency reported using smooth $\mathrm{SiO}_{2} \mathrm{NPs}$. To further increase the nucleation efficiency, it was anticipated that more than a grafted polymer shell on the $\mathrm{SiO}_{2} \mathrm{NPs}$ was required. 


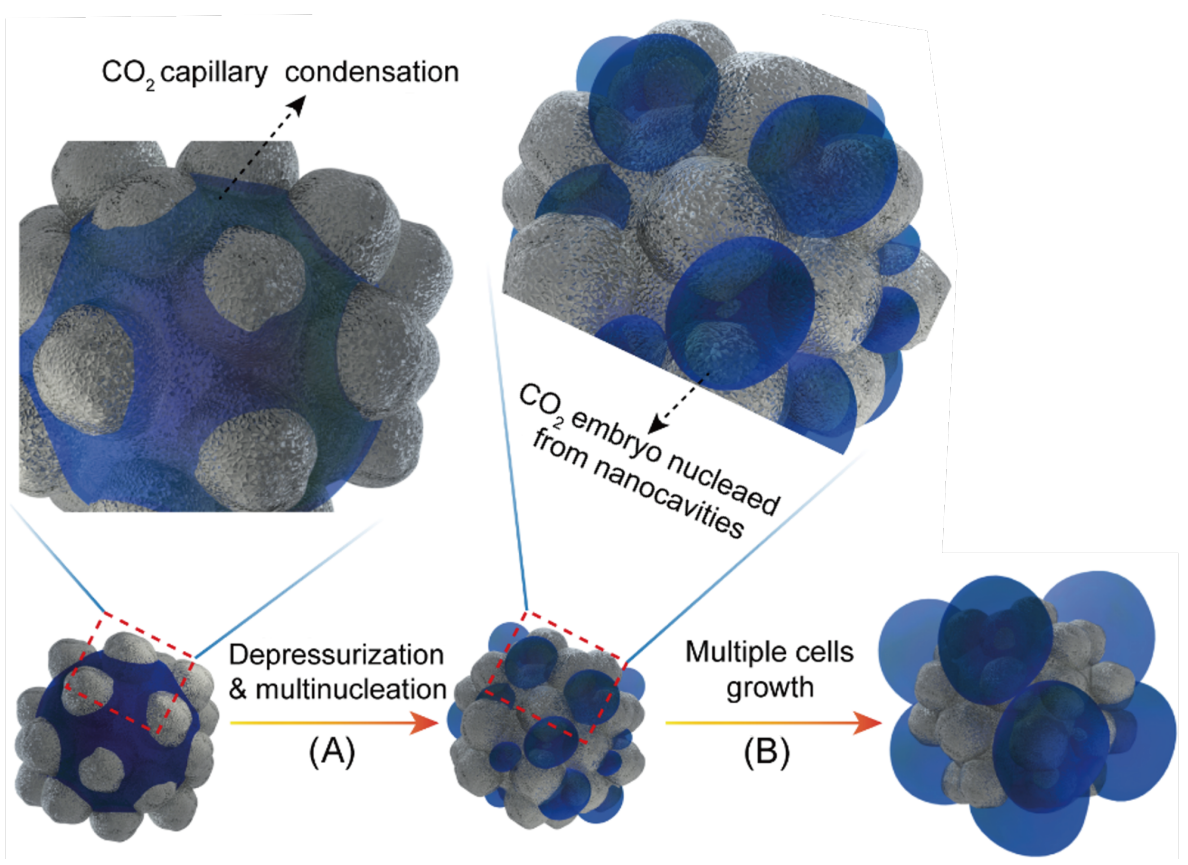

Figure 2.19 Schematic representation of the nucleation of multiple cells from a PDMS coated $\mathrm{SiO}_{2}-\mathrm{R}$. The saturation pressure and foaming temperature were 55 bar and $40^{\circ} \mathrm{C}$, respectively. Picture was adapted from reference ${ }^{263}$.

The thermodynamic model reported by Liu et al., ${ }^{240}$ directed us towards lowering the critical nucleation energy of nucleating particles by introducing surface-exposed nanocavities. Recently, we successfully exploited $\mathrm{SiO}_{2}-\mathrm{R} \mathrm{NPs}$ as nano-filler to boost the nucleation efficiency of nanoparticles in polymer foaming. ${ }^{263}$ We successfully synthesized PDMS coated $\mathrm{SiO}_{2}-\mathrm{R}$ NPs ( $\mathrm{SiO}_{2}-\mathrm{RP} \mathrm{NPs}$ ) with a diameter ranging from 80 to $200 \mathrm{~nm}$. For the $\mathrm{CO}_{2}$-based batch foaming of PMMA, the added $\mathrm{SiO}_{2}-\mathrm{RP}$ NPs had a nucleation efficiency of up to 6.2 , meaning that each particle was able to nucleate more than one foam cell. The significant increase in nucleation efficiency is ascribed to the capillary condensation of the physical blowing agent $\left(\mathrm{CO}_{2}\right)$ inside the nanocavities with the presence of a thin PDMS shell (favorable for nucleation), resulting in locally increased concentrations of $\mathrm{CO}_{2}$ (see Figure 2.19). The increased blowing agent concentration in the energetically favorable concave nucleation sites is responsible for the significantly enhanced nucleation efficiency of $\mathrm{SiO}_{2}-\mathrm{RP}$ NPs. It is believed that further tailoring the morphology of surface-engineered $\mathrm{SiO}_{2} \mathrm{NPs}$ in combination with different thermoplastic foam matrixes will open up new avenues for nanocellular foam morphology control. 


\subsection{Catalytic Effects of Silica Nanoparticles}

A catalyst is defined as a substance that is added to chemical reactions to increase the reaction rate without modifying the overall standard Gibbs energy change in the reaction. ${ }^{267}$ Usually, the catalyst provides an alternative reaction pathway/mechanism with lower activation energy while leaving the reaction equilibrium intact (see Figure 2.20). Catalysts are generally classified into three categories: homogeneous, heterogeneous, and enzymatic.

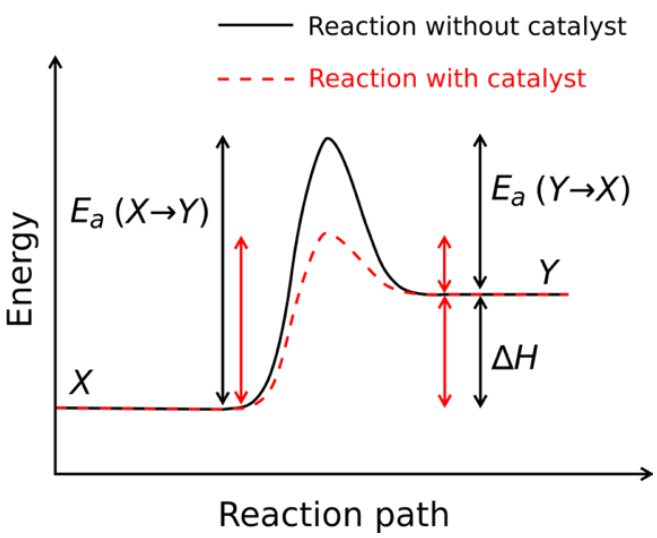

Figure 2.20 Energy profile of an endothermic reaction showing the reactants $(X)$, products $(Y)$, activation energy $(E a)$, and enthalpy $(\Delta H){ }^{268}$

\subsubsection{Catalytic Activities of Silica Nanoparticles}

NPs commonly have many unsaturated atoms at the surface and edge due to the unprecedented large surface/volume ratio. These unsaturated atoms can be easily coordinated with the surrounding species, making them ideal candidates for catalytical applications. NP catalysts can maximize exposure of active sites, like a homogeneous catalyst, while retaining the possibility to be separated, purified, and reused like a heterogeneous catalyst.

Noble metal NPs (NMNs), just like noble metals, are a fascinating class of materials in catalysis science because of their outstanding properties. ${ }^{269-272}$ The NMN catalysts commonly suffer from sintering, which can severally influence the catalytic performance of NMN catalysts. Using a support material is an effective approach to prevent NMNs from sintering.

As mentioned, $\mathrm{SiO}_{2} \mathrm{NPs}$ are commonly used as support material for $\mathrm{NMN}$. $\mathrm{SiO}_{2} \mathrm{NPs}$ are well known for their unique properties, such as i) facile synthesis, even at room 
temperature, ii) a size that can be easily tuned, iii) non-toxic and inexpensive, and iv) that they are stable in various organic solvents and at relatively high temperatures. Numerous efforts have been made to optimize the catalytic activity of NMN supported by $\mathrm{SiO}_{2} \mathrm{NPs}$. For example, Song et al. ${ }^{273}$ reported the synthesis of SBA-15 (mesoporous silica)supported 1-7 nm platinum (Pt) NPs via sonication and calcination at elevated temperatures. The resulting SBA-15 supported Pt NPs were tested for ethylene hydrogenation under variant conditions. The SBA-15 supported Pt NPs were stable under 20 torr $\mathrm{C}_{2} \mathrm{H}_{4}, 200$ torr $\mathrm{H}_{2}$, and $658 \mathrm{~K}$.

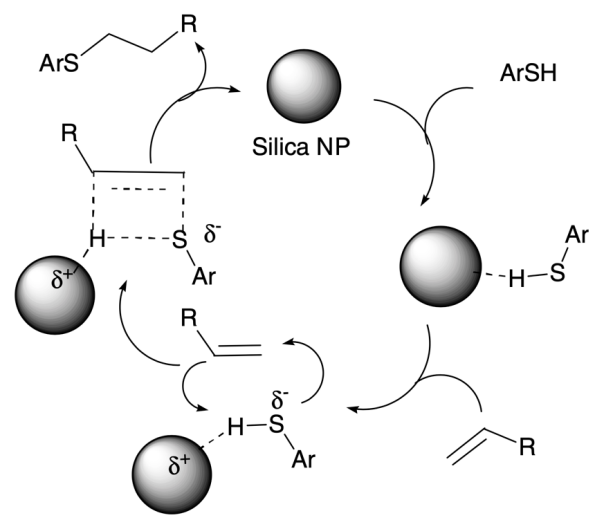

Figure 2.21 The proposed mechanism of $\mathrm{SiO}_{2}$ catalyzed linear thioether fabrication. ${ }^{274}$

As widely as $\mathrm{SiO}_{2} \mathrm{NP}$ is used as a support material for NMN, the catalytic activity of $\mathrm{SiO}_{2} \mathrm{NP}$ itself has rarely been presented and discussed in the literature. Banerjee et al. ${ }^{274}$ were among the pioneers to exploit the catalytic activity of $\mathrm{SiO}_{2}$ in organic transformations. They discussed that Stöber $\mathrm{SiO}_{2}$ NPs catalyzed anti-Markovnikov addition of thiols to alkenes and alkynes (see Figure 2.21). With the addition of $1 \mathrm{wt} \%$ $\mathrm{SiO}_{2} \mathrm{NPs}$, the thioethers can be produced at room temperature with a yield of up to $98 \%$ in 1.5 hours (see Figure 2.22). It usually takes more than 24 hours for the neat reaction to achieve the same yield. The same authors also utilized the catalytic activity of $\mathrm{SiO}_{2} \mathrm{NPs}$ in the bis-Michael addition of active methylene compounds to conjugated alkenes, ${ }^{275}$ which was considered possible only with a few catalysts, like ruthenium complex ${ }^{276}$ and basic ionic liquid catalyst ${ }^{277,278}$. Kundu et al. ${ }^{279}$ reported using a commercial silica gel for dithioethers synthesis. By controlling the moisture level of the silica gel (dry/moistened), the authors were able to conduct the regioselective synthesis of 1,2- and 1,3-dithioethers at room temperature by reacting a variety of aromatic thiols with alkyl bromide. Similarly, Nikoofar and Khalili used commercial $\mathrm{SiO}_{2}$ to catalyze the one-pot synthesis of symmetrical and unsymmetrical 3,3-di(aryl)indolin-2-ones. ${ }^{280}$ The synthesis was performed under solvent-free conditions with magnetic stirring or grinding at room 48 
temperature. Ramazani et al. ${ }^{281}$ discussed using $\mathrm{SiO}_{2}$ NPs to prepare ketoamide derivatives at ambient temperature. The ketoamide derivatives were prepared via a onepot multicomponent condensation reaction catalyzed by $\mathrm{SiO}_{2} \mathrm{NPs}$.

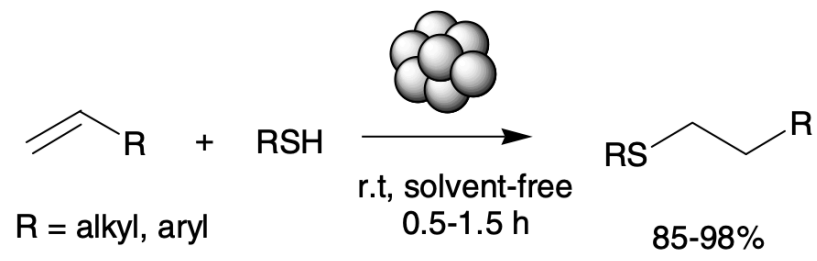

Figure 2.22 The silica NPs-catalyzed synthesis of linear thioethers. ${ }^{274}$

Besides the $\mathrm{SiO}_{2}$ catalyzed chemical synthesis, there is also literature that describes the use of mesoporous $\mathrm{SiO}_{2}$ to catalyze polymer degradation. The common catalysts for thermal degradation of polymers (e.g., polyethylene, polypropylene, polystyrene) into fuel oil are silica-alumina ${ }^{282-286}$, zeolites ${ }^{283,285,287-289}$, fluid catalytic cracking (FCC) catalysts $^{290-292}$, metal-based catalysts ${ }^{293}$, and basic oxides ${ }^{294}$.

In 1997, Sakata et al. reported using a non-conventional catalyst - mesoporous silica (KFS-16B) - to catalyze polyethylene degradation. ${ }^{295}$ The sodium silicate powder was calcined to form $\delta-\mathrm{Na}_{2} \mathrm{Si}_{2} \mathrm{O}_{5}$, followed by suspension in water and subsequent filtration to form a wet Kanemite $^{\circledR}$ paste. Then the paste was dispersed in hexadecyltrimethylammoniumbromide solution for ion exchange at the desired $\mathrm{pH}$ at 70 ${ }^{\circ} \mathrm{C}$, followed by calcination to form KFS-16B. ${ }^{296}$ The hexagonal pores $(\varnothing 3.6 \mathrm{~nm})$ on the KFS-16B particles acted as nanometric reactor vessels. They could encapsulate free radicals and extend their active time, therefore accelerating the degradation of polyethylene. In later research, Sakata et al. compared the catalytic activity of mesoporous $\mathrm{SiO}_{2}$ NPs and a solid acidic catalyst to degrade polyethylene and polypropylene into fuel oil. ${ }^{285}$ Instead of participating in the chemical reaction (i.e. polyethylene degradation), mesoporous $\mathrm{SiO}_{2}$ NPs acted as a reaction flask. The role of mesoporous $\mathrm{SiO}_{2}$ NPs during these chemical reactions did not fit the classical catalyst description. However, the presence of mesoporous $\mathrm{SiO}_{2}$ NPs decreased the reaction temperature, increased the yield, and shortened the time to reach equilibrium. The examples presented and discussed above show the potential of Si-containing NPs as a non-conventional catalyst. 


\subsection{Concluding Remarks}

Si-containing nanoparticles are an exciting and promising class of materials with countless applications. This is mainly ascribed to their (size-dependent) unique properties, low toxicity, and well-established synthetic preparation and derivatization methods. This Chapter presented and discussed the emerging fields of printable silicon nanoparticle-based electronics, theranostics, nanocellular polymer foaming, and catalysis. We recognize that at the forefront in these areas, the use of $\mathrm{SiNPs}_{\mathrm{N}}$ or $\mathrm{SiO}_{2} \mathrm{NPs}$ offers distinguished advantages over existing competing particles. We also identified that the main bottlenecks to solve are their large-scale affordable and reproducible production and legislation to enable biomedical applications. Provided that these challenges will be tackled, SiNPs and $\mathrm{SiO}_{2} \mathrm{NPs}$ are expected to become enabling platform particles that will enhance the development of the discussed emerging research areas to appropriate industrial levels.

\subsection{References}

(1) Osborne, I.; Lavine, M.; Coontz, R. Materials for Electronics. Looking beyond Silicon. Introduction. Science (New York, N.Y.) 2010, 327 (5973), 1595.

(2) Venema, L. Silicon Electronics and Beyond. Nature 2011, 479 (7373), 309.

(3) Orton, J. (2009). Semiconductors and the Information Revolution. Academic Press.

(4) Capek, I. (2019) Nanotechnology and Nanomaterials. In I. Capek (Ed.). Nanocomposite Structures and Dispersion (pp 1-93). Elsevier: Amsterdam.

(5) Sailor, M. J. (2012). Porous Silicon in Practice: Preparation, Characterization and Applications. Wiley-VCH.

(6) Dasog, M.; Kehrle, J.; Rieger, B.; Veinot, J. G. C. Silicon Nanocrystals and Silicon-Polymer Hybrids: Synthesis, Surface Engineering, and Applications. Angewandte Chemie - International Edition 2016, 55 (7), 2322-2339.

(7) Jeelani, P. G.; Mulay, P.; Venkat, R.; Ramalingam, C. Multifaceted Application of Silica Nanoparticles. A Review. Silicon 2020, 12 (6), 1337-1354.

(8) Narayan, R.; Nayak, U. Y.; Raichur, A. M.; Garg, S. Mesoporous Silica Nanoparticles: A Comprehensive Review on Synthesis and Recent Advances. Pharmaceutics 2018, 10 (3), 118.

(9) Chang, H.; Sun, S. Q. Silicon Nanoparticles: Preparation, Properties, and Applications. Chinese Physics B 2014, 23 (8), 1-14.

(10) Don, M. M.; San, C. Y.; Jeevanandam, J. (2016) Antimicrobial Properties of Nanobiomaterials and the Mechanism. In A. Grumezescu (Ed.). Nanobiomaterials in Antimicrobial Therapy: Applications of Nanobiomaterials (pp 261-312). William Andrew.

(11) Popelensky, V. M.; Dorofeev, S. G.; Kononov, N. N.; Bubenov, S. S.; Vinokurov, A. A. Room Temperature Oxidation of Si Nanocrystals at Dry and Wet Air. 
Journal of Nanoparticle Research 2020, 22 (3), 54.

(12) Belomoin, G.; Therrien, J.; Smith, A.; Rao, S.; Twesten, R.; Chaieb, S.; Nayfeh, M. H.; Wagner, L.; Mitas, L. Observation of a Magic Discrete Family of Ultrabright Si Nanoparticles. Applied Physics Letters 2002, 80 (5), 841-843.

(13) Canham, L. T. Silicon Quantum Wire Array Fabrication by Electrochemical and Chemical Dissolution of Wafers. Applied Physics Letters 1990, 57 (10), 10461048.

(14) Dohnalová, K.; Fučíková, A.; Umesh, C. P.; Humpolíčková, J.; Paulusse, J. M. J.; Valenta, J.; Zuilhof, H.; Hof, M.; Gregorkiewicz, T. Microscopic Origin of the Fast Blue-Green Luminescence of Chemically Synthesized Non-Oxidized Silicon Quantum Dots. Small 2012, 8 (20), 3185-3191.

(15) Wen, X.; Zhang, P.; Smith, T. A.; Anthony, R. J.; Kortshagen, U. R.; Yu, P.; Feng, Y.; Shrestha, S.; Coniber, G.; Huang, S. Tunability Limit of Photoluminescence in Colloidal Silicon Nanocrystals. Scientific Reports 2015, 5 (1), 12469.

(16) Sinelnikov, R.; Dasog, M.; Beamish, J.; Meldrum, A.; Veinot, J. G. C. Revisiting an Ongoing Debate: What Role Do Surface Groups Play in Silicon Nanocrystal Photoluminescence? ACS Photonics 2017, 4 (8), 1920-1929.

(17) Meier, C.; Gondorf, A.; Lüttjohann, S.; Lorke, A.; Wiggers, H. Silicon Nanoparticles: Absorption, Emission, and the Nature of the Electronic Bandgap. Journal of Applied Physics 2007, 101 (10), 103112.

(18) Shirahata, N. Colloidal Si Nanocrystals: A Controlled Organic-Inorganic Interface and Its Implications of Color-Tuning and Chemical Design toward Sophisticated Architectures. Physical Chemistry Chemical Physics 2011, 13 (16), 7284-7294.

(19) Gonzalez, C. M.; Iqbal, M.; Dasog, M.; Piercey, D. G.; Lockwood, R.; Klapötke, T. M.; Veinot, J. G. C. Detection of High-Energy Compounds Using Photoluminescent Silicon Nanocrystal Paper Based Sensors. Nanoscale 2014, 6 (5), 2608-2612.

(20) Sailor, M. J.; Wu, E. C. Photoluminescence-Based Sensing with Porous Silicon Films, Microparticles, and Nanoparticles. Advanced Functional Materials 2009, 19 (20), 3195-3208.

(21) Peng, F.; Su, Y.; Zhong, Y.; Fan, C.; Lee, S. T.; He, Y. Silicon Nanomaterials Platform for Bioimaging, Biosensing, and Cancer Therapy. Accounts of Chemical Research 2014, 47 (2), 612-623.

(22) Rosso-Vasic, M.; Spruijt, E.; Popović, Z.; Overgaag, K.; Van Lagen, B.; Grandidier, B.; Vanmaekelbergh, D.; Domínguez-Gutiérrez, D.; De Cola, L.; Zuilhof, H. Amine-Terminated Silicon Nanoparticles: Synthesis, Optical Properties and Their Use in Bioimaging. Journal of Materials Chemistry 2009, 19 (33), 5926-5933.

(23) Maier-Flaig, F.; Rinck, J.; Stephan, M.; Bocksrocker, T.; Bruns, M.; Kübel, C.; Powell, A. K.; Ozin, G. A.; Lemmer, U. Multicolor Silicon Light-Emitting Diodes (SiLEDs). Nano Letters 2013, 13 (2), 475-480.

(24) Stupca, M.; Alsalhi, M.; Al Saud, T.; Almuhanna, A.; Nayfeh, M. H. Enhancement of Polycrystalline Silicon Solar Cells Using Ultrathin Films of Silicon Nanoparticle. Applied Physics Letters 2007, 91 (6), 063107.

(25) Cullis, A. G.; Canham, L. T. Visible Light Emission Due to Quantum Size Effects in Highly Porous Crystalline Silicon. Nature 1991, 353 (6342), 335-338. 
(26) Akkerman, Q. A. (2019). Lead Halide Perovskite Nanocrystals - A New Age of Semiconductor Nanocrystals. Università degli Studi di Genova. https://iris.unige.it/handle/11567/941201

(27) Alexander, G. B.; Heston, W. M.; Iler, R. K. The Solubility of Amorphous Silica in Water. Journal of Physical Chemistry 1954, 58 (6), 453-455.

(28) Shen, D.; Yang, J.; Li, X.; Zhou, L.; Zhang, R.; Li, W.; Chen, L.; Wang, R.; Zhang, F.; Zhao, D. Biphase Stratification Approach to Three-Dimensional Dendritic Biodegradable Mesoporous Silica Nanospheres. Nano Letters 2014, 14 (2), $923-$ 932.

(29) Argyo, C.; Weiss, V.; Bräuchle, C.; Bein, T. Multifunctional Mesoporous Silica Nanoparticles as a Universal Platform for Drug Delivery. Chemistry of Materials 2014, 26 (1), 435-451.

(30) Giri, S.; Trewyn, B. G.; Stellmaker, M. P.; Lin, V. S. Y. Stimuli-Responsive Controlled-Release Delivery System Based on Mesoporous Silica Nanorods Capped with Magnetic Nanoparticles. Angewandte Chemie - International Edition 2005, 44 (32), 5038-5044.

(31) Barbé, C.; Bartlett, J.; Kong, L.; Finnie, K.; Lin, H. Q.; Larkin, M.; Calleja, S.; Bush, A.; Calleja, G. Silica Particles: A Novel Drug-Delivery System. Advanced Materials 2004, 16 (21), 1959-1966.

(32) Dave, B. C.; Dunn, B.; Valentine, J. S.; Zink, J. I. Sol-Gel Encapsulation Methods for Biosensors. Analytical Chemistry 1994, 66 (22), 1120A-1127A.

(33) Lin, V. S. Y.; Lai, C. Y.; Huang, J.; Song, S. A.; Xu, S. Molecular Recognition inside of Multifunctionalized Mesoporous Silicas: Toward Selective Fluorescence Detection of Dopamine and Glucosamine. Journal of the American Chemical Society 2001, 123 (46), 11510-11511.

(34) Santra, S.; Malhotra, A. Fluorescent Nanoparticle Probes for Imaging of Cancer. Wiley Interdisciplinary Reviews: Nanomedicine and Nanobiotechnology 2011, 3 (5), 501-510.

(35) He, X.; Duan, J.; Wang, K.; Tan, W.; Lin, X.; He, C. A Novel Fluorescent Label Based on Organic Dye-Doped Silica Nanoparticles for HepG Liver Cancer Cell Recognition. Journal of Nanoscience and Nanotechnology 2004, 4 (6), 585-589.

(36) Yao, G.; Wang, L.; Wu, Y.; Smith, J.; Xu, J.; Zhao, W.; Lee, E.; Tan, W. FloDots: Luminescent Nanoparticles. Analytical and Bioanalytical Chemistry 2006, 385 (3), 518-524.

(37) Wang, L.; Yang, C.; Tan, W. Dual-Luminophore-Doped Silica Nanoparticles for Multiplexed Signaling. Nano Letters 2005, 5 (1), 37-43.

(38) Zhao, X.; Tapec-Dytioco, R.; Tan, W. Ultrasensitive DNA Detection Using Highly Fluorescent Bioconjugated Nanoparticles. Journal of the American Chemical Society 2003, 125 (38), 11474-11475.

(39) Ulrich, G. D.; Rieh, J. W. Aggregation and Growth of Submicron Oxide Particles in Flames. Journal of Colloid And Interface Science 1982, 87 (1), 257-265.

(40) Chen, X.; Jiang, J.; Yan, F.; Tian, S.; Li, K. A Novel Low Temperature Vapor Phase Hydrolysis Method for the Production of Nano-Structured Silica Materials Using Silicon Tetrachloride. RSC Advances 2014, 4 (17), 8703-8710.

(41) Jal, P.K.; Sudarshan, M.; Saha, A.; Patel, S.; Mishra, B.K. Synthesis and Characterization of Nanosilica Prepared by Precipitation Method. Colloids and 
Surfaces A: Physicochemical and Engineering Aspects 2004, 240 (1-3), 173-178.

(42) Musić, S.; Filipović-Vinceković, N.; Sekovanić, L. Precipitation of Amorphous $\mathrm{SiO}_{2}$ Particles and Their Properties. Brazilian Journal of Chemical Engineering 2011, 28 (1), 89-94.

(43) Wang, X. D.; Shen, Z. X.; Sang, T.; Cheng, X. bin; Li, M. F.; Chen, L. Y.; Wang, Z. S. Preparation of Spherical Silica Particles by Stöber Process with High Concentration of Tetra-Ethyl-Orthosilicate. Journal of Colloid and Interface Science 2010, 341 (1), 23-29.

(44) Canton, G.; Riccò, R.; Marinello, F.; Carmignato, S.; Enrichi, F. Modified Stöber Synthesis of Highly Luminescent Dye-Doped Silica Nanoparticles. Journal of Nanoparticle Research 2011, 13 (9), 4349-4356.

(45) Stöber, W.; Fink, A.; Bohn, E. Controlled Growth of Monodisperse Silica Spheres in the Micron Size Range. Journal of Colloid And Interface Science 1968, 26 (1), 62-69.

(46) Santamaría Razo, D. A.; Pallavidino, L.; Garrone, E.; Geobaldo, F.; Descrovi, E.; Chiodoni, A.; Giorgis, F. A Version of Stöber Synthesis Enabling the Facile Prediction of Silica Nanospheres Size for the Fabrication of Opal Photonic Crystals. Journal of Nanoparticle Research 2008, 10 (7), 1225-1229.

(47) Nozawa, K.; Gailhanou, H.; Raison, L.; Panizza, P.; Ushiki, H.; Sellier, E.; Delville, J. P.; Delville, M. H. Smart Control of Monodisperse Stöber Silica Particles: Effect of Reactant Addition Rate on Growth Process. Langmuir 2005, $21(4), 1516-1523$.

(48) Grün, M.; Lauer, I.; Unger, K. K. The Synthesis of Micrometer- and Submicrometer-Size Spheres of Ordered Mesoporous Oxide MCM-41. Advanced Materials 1997, 9 (3), 254-257.

(49) Gao, C.; Qiu, H.; Zeng, W.; Sakamoto, Y.; Terasaki, O.; Sakamoto, K.; Chen, Q.; Che, S. Formation Mechanism of Anionic Surfactant-Templated Mesoporous Silica. Chemistry of Materials 2006, 18 (16), 3904-3914.

(50) Kumar, D.; Schumacher, K.; du Fresne von Hohenesche, C.; Grün, M.; Unger, K. K. MCM-41, MCM-48 and Related Mesoporous Adsorbents: Their Synthesis and Characterisation. Colloids and Surfaces A: Physicochemical and Engineering Aspects 2001, 187-188, 109-116.

(51) Flodström, K.; Wennerström, H.; Alfredsson, V. Mechanism of Mesoporous Silica Formation, a Time-Resolved NMR and TEM Study of Silica-Block Copolymer Aggregation. Langmuir 2004, 20 (3), 680-688.

(52) Jammaer, J.; Aerts, A.; D’Haen, J.; Seo, J. W.; Martens, J. A. Convenient Synthesis of Ordered Mesoporous Silica at Room Temperature and Quasi-Neutral PH. Journal of Materials Chemistry 2009, 19 (44), 8290-8293.

(53) Lam, C.; Zhang, Y. F.; Tang, Y. H.; Lee, C. S.; Bello, I.; Lee, S. T. Large-Scale Synthesis of Ultrafine Si Nanoparticles by Ball Milling. Journal of Crystal Growth 2000, 220 (4), 466-470.

(54) Yoshida, T.; Takeyama, S.; Yamada, Y.; Mutoh, K. Nanometer-Sized Silicon Crystallites Prepared by Excimer Laser Ablation in Constant Pressure Inert Gas. Applied Physics Letters 1996, 68 (13), 1772-1774.

(55) Ahire, J. H.; Wang, Q.; Coxon, P. R.; Malhotra, G.; Brydson, R.; Chen, R.; Chao, Y. Highly Luminescent and Nontoxic Amine-Capped Nanoparticles from Porous 
Silicon: Synthesis and Their Use in Biomedical Imaging. ACS Applied Materials and Interfaces 2012, 4 (6), 3285-3292.

(56) Wilcoxon, J. P.; Samara, G. A.; Provencio, P. N. Optical and Electronic Properties of Si Nanoclusters Synthesized in Inverse Micelles. Physical Review B Condensed Matter and Materials Physics 1999, 60 (4), 2704-2714.

(57) Heath, J. R. A Liquid-Solution-Phase Synthesis of Crystalline Silicon. Science 1992, 258 (5085), 1131-1133.

(58) Knipping, J.; Wiggers, H.; Rellinghaus, B.; Roth, P.; Konjhodzic, D.; Meier, C. Synthesis of High Purity Silicon Nanoparticles in a Low Pressure Microwave Reactor. Journal of Nanoscience and Nanotechnology 2004, 4 (8), 1039-1044.

(59) Ehbrecht, M.; Huisken, F. Gas-Phase Characterization of Silicon Nanoclusters Produced by Laser Pyrolysis of Silane. Physical Review B - Condensed Matter and Materials Physics 1999, 59 (4), 2975-2985.

(60) Hülser, T.; Schnurre, S. M.; Wiggers, H.; Schulz, C. Gas-Phase Synthesis of Nanoscale Silicon as an Economical Route towards Sustainable Energy Technology. KONA Powder and Particle Journal 2011, 29 (29), 191-207.

(61) Smokefoot. highly simplified representation of the condensation of TEOS in sol gel

https://commons.wikimedia.org/wiki/File:SolGelCartoon.png.

(62) Wu, Y.; Zhong, Y.; Chu, B.; Sun, B.; Song, B.; Wu, S.; Su, Y.; He, Y. PlantDerived Fluorescent Silicon Nanoparticles Featuring Excitation WavelengthDependent Fluorescence Spectra for Anti-Counterfeiting Applications. Chemical Communications 2016, 52 (43), 7047-7050.

(63) Pratsinis, S. E. Flame Aerosol Synthesis of Ceramic Powders. Progress in Energy and Combustion Science 1998, 24 (3), 197-219.

(64) Khavryutchenko, V.; Khavryutchenko, A.; Barthel, H. Fumed Silica Synthesis: Influence of Small Molecules on the Particle Formation Process. Macromolecular Symposia 2001, 169 (1), 1-6.

(65) Rahman, I. A.; Padavettan, V. Synthesis of Silica Nanoparticles by Sol-Gel: SizeDependent Properties, Surface Modification, and Applications in Silica-Polymer Nanocompositesa Review. Journal of Nanomaterials 2012, 2012, 132424.

(66) Gorji, B.; Fazaeli, R.; Niksirat, N. Synthesis and Characterizations of Silica Nanoparticles by a New Sol-Gel Method. Quarterly Journal of Applied Chemical Research 2012, 6 (3), 22-26.

(67) Zulfiqar, U.; Subhani, T.; Husain, S. W. Synthesis and Characterization of Silica Nanoparticles from Clay. Journal of Asian Ceramic Societies 2016, 4 (1), 91-96.

(68) Cummins, G.; Desmulliez, M. P. Y. Inkjet Printing of Conductive Materials: A Review. Circuit World 2012, 38 (4), 193-213.

(69) Yin, Z. P.; Huang, Y. A.; Bu, N. bin; Wang, X. M.; Xiong, Y. L. Inkjet Printing for Flexible Electronics: Materials, Processes and Equipments. Chinese Science Bulletin 2010, 55 (30), 3383-3407.

(70) Andò, B.; Baglio, S. Inkjet-Printed Sensors: A Useful Approach for Low Cost, Rapid Prototyping [Instrumentation Notes]. IEEE Instrumentation and Measurement Magazine 2011, 14 (5), 36-40.

(71) Cruz, S. M. F.; Rocha, L. A.; Viana, J. C. (2018). Printing Technologies on Flexible Substrates for Printed Electronics. In S. Rackauskas (Ed.). Flexible 
Electronics. IntechOpen. doi: 10.5772/intechopen.76161.

(72) Cauchois, R.; Saadaoui, M.; Yakoub, A.; Inal, K.; Dubois-Bonvalot, B.; Fidalgo, J. C. Impact of Variable Frequency Microwave and Rapid Thermal Sintering on Microstructure of Inkjet-Printed Silver Nanoparticles. Journal of Materials Science 2012, 47 (20), 7110-7116.

(73) Yakoub, A.; Saadaoui, M.; Benaben, P.; Iliev, P. High Efficiency of Low-Cost Spiral Antennas for RFID, Fully Printed by an Inline Inkjet Process. Proceddings Large Organic and Printed Electronics Conference, 2012.

(74) Sanaur, S.; Whalley, A.; Alameddine, B.; Carnes, M.; Nuckolls, C. Jet-Printed Electrodes and Semiconducting Oligomers for Elaboration of Organic Thin-Film Transistors. Organic Electronics 2006, 7 (5), 423-427.

(75) Kamyshny, A. Metal-Based Inkjet Inks for Printed Electronics. The Open Applied Physics Journal 2011, 4 (1), 19-36.

(76) Derby, B. Inkjet Printing of Functional and Structural Materials: Fluid Property Requirements, Feature Stability, and Resolution. Annual Review of Materials Research 2010, 40, 395-414.

(77) Caironi, M.; Gili, E.; Sakanoue, T.; Cheng, X.; Sirringhaus, H. High Yield, Single Droplet Electrode Arrays for Nanoscale Printed Electronics. ACS Nano 2010, 4 (3), 1451-1456.

(78) Wang, J.; Cheng, X.; Caironi, M.; Gao, F.; Yang, X.; Greenham, N. C. Entirely Solution-Processed Write-Once-Read-Many-Times Memory Devices and Their Operation Mechanism. Organic Electronics 2011, 12 (7), 1271-1274.

(79) Mizukami, M.; Cho, S. Il; Watanabe, K.; Abiko, M.; Suzuri, Y.; Tokito, S.; Kido, J. Flexible Organic Light-Emitting Diode Displays Driven by Inkjet-Printed HighMobility Organic Thin-Film Transistors. IEEE Electron Device Letters 2018, 39 (1), 39-42.

(80) Templier, F.; Aventurier, B.; Demars, P.; Botrel, J. L.; Martin, P. Fabrication of High Performance Low Temperature Poly-Silicon Backplanes on Metal Foil for Flexible Active-Matrix Organic Light Emission Diode Displays. Thin Solid Films 2007, 515 (19 SPEC. ISS.), 7428-7432.

(81) Kukkola, J.; Mohl, M.; Leino, A. R.; Tóth, G.; Wu, M. C.; Shchukarev, A.; Popov, A.; Mikkola, J. P.; Lauri, J.; Riihimäki, M.; Lappalainen, J.; Jantunen, H.; Kordás, K. Inkjet-Printed Gas Sensors: Metal Decorated WO 3 Nanoparticles and Their Gas Sensing Properties. Journal of Materials Chemistry 2012, 22 (34), 1787817886.

(82) Li, B.; Santhanam, S.; Schultz, L.; Jeffries-EL, M.; Iovu, M. C.; Sauvé, G.; Cooper, J.; Zhang, R.; Revelli, J. C.; Kusne, A. G.; Snyder, J. L.; Kowalewski, T.; Weiss, L. E.; McCullough, R. D.; Fedder, G. K.; Lambeth, D. N. Inkjet Printed Chemical Sensor Array Based on Polythiophene Conductive Polymers. Sensors and Actuators, B: Chemical 2007, 123 (2), 651-660.

(83) Komuro, N.; Takaki, S.; Suzuki, K.; Citterio, D. Inkjet Printed (Bio)Chemical Sensing Devices. Analytical and Bioanalytical Chemistry 2013, 405 (17), 57855805.

(84) Noh, Y. Y.; Zhao, N.; Caironi, M.; Sirringhaus, H. Downscaling of Self-Aligned, All-Printed Polymer Thin-Film Transistors. Nature Nanotechnology 2007, 2 (12), 784-789. 
(85) Minemawari, H.; Yamada, T.; Matsui, H.; Tsutsumi, J. Y.; Haas, S.; Chiba, R.; Kumai, R.; Hasegawa, T. Inkjet Printing of Single-Crystal Films. Nature 2011, 475 (7356), 364-367.

(86) Baeg, K. J.; Khim, D.; Kim, J.; Kim, D. Y.; Sung, S. W.; Yang, B. Do; Noh, Y. Y. Flexible Complementary Logic Gates Using Inkjet-Printed Polymer FieldEffect Transistors. IEEE Electron Device Letters 2013, 34 (1), 126-128.

(87) Molina-Lopez, F.; Gao, T. Z.; Kraft, U.; Zhu, C.; Öhlund, T.; Pfattner, R.; Feig, V. R.; Kim, Y.; Wang, S.; Yun, Y.; Bao, Z. Inkjet-Printed Stretchable and Low Voltage Synaptic Transistor Array. Nature Communications 2019, 10 (1), 1-10.

(88) Sekitani, T.; Noguchi, Y.; Zschieschang, U.; Klauk, H.; Someya, T. Organic Transistors Manufactured Using Inkjet Technology with Subfemtoliter Accuracy. Proceedings of the National Academy of Sciences of the United States of America 2008, 105 (13), 4976-4980.

(89) Lawes, S.; Sun, Q.; Lushington, A.; Xiao, B.; Liu, Y.; Sun, X. Inkjet-Printed Silicon as High Performance Anodes for Li-Ion Batteries. Nano Energy 2017, 36, 313-321.

(90) Hoth, C. N.; Schilinsky, P.; Choulis, S. A.; Brabec, C. J. Printing Highly Efficient Organic Solar Cells. Nano Letters 2008, 8 (9), 2806-2813.

(91) Teichler, A.; Eckardt, R.; Hoeppener, S.; Friebe, C.; Perelaer, J.; Senes, A.; Morana, M.; Brabec, C. J.; Schubert, U. S. Combinatorial Screening of Polymer: Fullerene Blends for Organic Solar Cells by Inkjet Printing. Advanced Energy Materials 2011, 1 (1), 105-114.

(92) Nayak, L.; Mohanty, S.; Nayak, S. K.; Ramadoss, A. A Review on Inkjet Printing of Nanoparticle Inks for Flexible Electronics. Journal of Materials Chemistry C 2019, 7 (29), 8771-8795.

(93) Vincent, B. The Effect of Adsorbed Polymers on Dispersion Stability. Advances in Colloid and Interface Science 1974, 4 (2-3), 193-277.

(94) Yu, W.; Xie, H. A Review on Nanofluids: Preparation, Stability Mechanisms, and Applications. Journal of Nanomaterials 2012, 2012, 435873.

Physical Stability of Disperse Systems https://lubrizolcdmo.com/technical-briefs/physical-stability-of-disperse-systems/ (accessed $2020-10-11$ ).

(96) Sailor, M. J. (2017). Chemical Reactivity and Surface Chemistry of Porous Silicon. In L. Canham (Ed.). Handbook of Porous Silicon (pp 1-20). Springer, Cham.

(97) Du, X.; He, J. A Self-Templated Etching Route to Surface-Rough Silica Nanoparticles for Superhydrophobic Coatings. ACS Applied Materials and Interfaces 2011, 3 (4), 1269-1276.

(98) Dehzangi, A.; Larki, F.; Majlis, B. B.; Naseri, M. G.; Navasery, M.; Abdullah, A. M.; Hutagalung, S. D.; Hamid, N. A.; Noor, M. M.; Vakilian, M.; Saion, E. B. Impact of $\mathrm{KOH}$ Etching on Nanostructure Fabricated by Local Anodic Oxidation Method. International Journal of Electrochemical Science 2013, 8 (6), 8084 8096.

(99) Seidel, H.; Csepregi, L.; Heuberger, A.; Baumgärtel, H. Anisotropic Etching of Crystalline Silicon in Alkaline Solutions: I . Orientation Dependence and Behavior of Passivation Layers. Journal of The Electrochemical Society 1990, 137 
(11), 3612-3626.

(100) Yin, S. Unpulished Data. For further reference, the reader is directed to referrence 97, which is reporting the ethcing of planar Si surface with $\mathrm{KOH}$.

(101) Ogata, Y.; Niki, H.; Sakka, T.; Iwasaki, M. Oxidation of Porous Silicon under Water Vapor Environment. Journal of The Electrochemical Society 1995, 142 (5), 1595-1601.

(102) Lehmann, V. The Chemical Dissolution of Silicon. Electrochemistry of Silicon 2003, 23-38.

(103) Benke, G.; Gnot, W. The Electrochemical Dissolution of Platinum. Hydrometallurgy 2002, 64, 205-218.

(104) Wheeler, L. M.; Neale, N. R.; Chen, T.; Kortshagen, U. R. Hypervalent Surface Interactions for Colloidal Stability and Doping of Silicon Nanocrystals. Nature Communications 2013, 4, 1-10.

(105) Erogbogbo, F.; Liu, T.; Ramadurai, N.; Tuccarione, P.; Lai, L.; Swihart, M. T.; Prasad, P. N. Creating Ligand-Free Silicon Germanium Alloy Nanocrystal Inks. ACS Nano 2011, 5 (10), 7950-7959.

(106) Kůsová, K.; Cibulka, O.; Dohnalová, K.; Pelant, I.; Fučíková, A.; Valenta, J. Yellow-Emitting Colloidal Suspensions of Silicon Nanocrystals: Fabrication Technology, Luminescence Performance and Application Prospects. Physica E: Low-Dimensional Systems and Nanostructures 2009, 41 (6), 982-985.

(107) Bywalez, R.; Karacuban, H.; Nienhaus, H.; Schulz, C.; Wiggers, H. Stabilization of Mid-Sized Silicon Nanoparticles by Functionalization with Acrylic Acid. Nanoscale Research Letters 2012, 7 (1), 76.

(108) Reindl, A.; Voronov, A.; Gorle, P. K.; Rauscher, M.; Roosen, A.; Peukert, W. Dispersing and Stabilizing Silicon Nanoparticles in a Low-Epsilon Medium. Colloids and Surfaces A: Physicochemical and Engineering Aspects 2008, 320 (1-3), 183-188.

(109) Boukherroub, R.; Wojtyk, J. T. C.; Wayner, D. D. M.; Lockwood, D. J. Thermal Hydrosilylation of Undecylenic Acid with Porous Silicon. Journal of The Electrochemical Society 2002, 149 (2), H59.

(110) Stewart, M. P.; Buriak, J. M. Photopatterned Hydrosilylation on Porous Silicon. Angewandte Chemie - International Edition 1998, 37 (23), 3257-3260.

(111) Buriak, J. M. Organometallic Chemistry on Silicon and Germanium Surfaces. Chemical Reviews 2002, 102 (5), 1271-1308.

(112) Linford, M. R.; Chidsey, C. E. D. Alkyl Monolayers Covalently Bonded to Silicon Surfaces. Journal of the American Chemical Society 1993, 115 (26), 1263112632.

(113) Purkait, T. K.; Iqbal, M.; Wahl, M. H.; Gottschling, K.; Gonzalez, C. M.; Islam, M. A.; Veinot, J. G. C. Borane-Catalyzed Room-Temperature Hydrosilylation of Alkenes/Alkynes on Silicon Nanocrystal Surfaces. Journal of the American Chemical Society 2014, 136 (52), 17914-17917.

(114) Buriak, J. M. Organometallic Chemistry on Silicon and Germanium Surfaces. Chemical Reviews 2002, 102 (5), 1271-1308.

(115) Kelly, J. A.; Veinot, J. G. C. An Investigation into Near-UV Hydrosilylation of Freestanding Silicon Nanocrystals. ACS Nano 2010, 4 (8), 4645-4656.

(116) Holman, Z. C.; Kortshagen, U. R. Nanocrystal Inks without Ligands: Stable 
Colloids of Bare Germanium Nanocrystals. Nano Letters 2011, 11 (5), 21332136.

(117) Erogbogbo, F.; Liu, T.; Ramadurai, N.; Tuccarione, P.; Lai, L.; Swihart, M. T.; Prasad, P. N. Creating Ligand-Free Silicon Germanium Alloy Nanocrystal Inks. ACS Nano 2011, 5 (10), 7950-7959.

(118) Holman, Z. C.; Kortshagen, U. R. Nanocrystal Inks without Ligands: Stable Colloids of Bare Germanium Nanocrystals. Nano Letters 2011, 11 (5), 21332136.

(119) Grubbs, R. B. Roles of Polymer Ligands in Nanoparticle Stabilization. Polymer Reviews 2007, 47 (2), 197-215.

(120) Witten, T. A.; Pincus, P. A.; Pincus, P. A. Colloid Stabilization by Long Grafted Polymers. Macromolecules 1986, 19 (10), 2509-2513.

(121) Milligan, W. O. Colloid Science. By James W. McBain. The Journal of Physical Chemistry 1951, 55 (7), 1277-1277.

(122) Zhao, B.; Brittain, W. J. Polymer Brushes: Surface-Immobilized Macromolecules. Progress in Polymer Science (Oxford) 2000, 25 (5), 677-710.

(123) Tsubokawa, N. Surface Grafting of Polymers onto Nanoparticles in a SolventFree Dry-System and Applications of Polymer-Grafted Nanoparticles as Novel Functional Hybrid Materials. Polymer Journal 2007, 39 (10), 983-1000.

(124) Mohammadi Sejoubsari, R.; Martinez, A. P.; Kutes, Y.; Wang, Z.; Dobrynin, A. V.; Adamson, D. H. "Grafting-Through": Growing Polymer Brushes by Supplying Monomers through the Surface. Macromolecules 2016, 49 (7), 2477 2483.

(125) Zoppe, J. O.; Ataman, N. C.; Mocny, P.; Wang, J.; Moraes, J.; Klok, H. A. Surface-Initiated Controlled Radical Polymerization: State-of-the-Art, Opportunities, and Challenges in Surface and Interface Engineering with Polymer Brushes. Chemical Reviews 2017, 117 (3), 1105-1318.

(126) Henze, M.; Mädge, D.; Prucker, O.; Rühe, J. "Grafting through”: Mechanistic Aspects of Radical Polymerization Reactions with Surface-Attached Monomers. Macromolecules 2014, 47 (9), 2929-2937.

(127) Lizundia, E.; Meaurio, E.; Vilas, J. L. (2016) Grafting of Cellulose Nanocrystals. In D. Puglia \& E. Fortunati \& J. M. Kenny (Ed.). Multifunctional Polymeric Nanocomposites Based on Cellulosic Reinforcements (pp 61-113). William Andrew.

(128) Braunecker, W. A.; Matyjaszewski, K. Controlled/Living Radical Polymerization: Features, Developments, and Perspectives. Progress in Polymer Science (Oxford) 2007, 32 (1), 93-146.

(129) Yan, J.; Bockstaller, M. R.; Matyjaszewski, K. Brush-Modified Materials: Control of Molecular Architecture, Assembly Behavior, Properties and Applications. Progress in Polymer Science 2020, 100, 101180.

(130) Matyjaszewski, K.; Xia, J. Atom Transfer Radical Polymerization. Chemical Reviews 2001, 101 (9), 2921-2990.

(131) Matyjaszewski, K. Atom Transfer Radical Polymerization (ATRP): Current Status and Future Perspectives. Macromolecules 2012, 45 (10), 4015-4039.

(132) Chiefari, J.; Chong, Y. K.; Ercole, F.; Krstina, J.; Jeffery, J.; Le, T. P. T.; Mayadunne, R. T. A.; Meijs, G. F.; Moad, C. L.; Moad, G.; Rizzardo, E.; Thang, 
S. H. Living Free-Radical Polymerization by Reversible Addition - Fragmentation Chain Transfer: The RAFT Process. Macromolecules 1998, 31 (16), 5559-5562.

(133) Moad, G.; Rizzardo, E.; Thang, S. H. Living Radical Polymerization by the RAFT Process. Australian Journal of Chemistry 2005, 58 (6), 379-410.

(134) Hawker, C. J.; Bosman, A. W.; Harth, E. New Polymer Synthesis by Nitroxide Mediated Living Radical Polymerizations. Chemical Reviews 2001, 101 (12), 3661-3688.

(135) Nicolas, J.; Guillaneuf, Y.; Lefay, C.; Bertin, D.; Gigmes, D.; Charleux, B. Nitroxide-Mediated Polymerization. Progress in Polymer Science 2013, 38 (1), 63-235.

(136) Georges, M. K.; Veregin, R. P. N.; Kazmaier, P. M.; Hamer, G. K. Narrow Molecular Weight Resins by a Free-Radical Polymerization Process. Macromolecules 1993, 26 (11), 2987-2988.

(137) Matyjaszewski, K. Advanced Materials by Atom Transfer Radical Polymerization. Advanced Materials 2018, 30 (23), 10.1002/adma.201706441.

(138) Von Werne, T.; Patten, T. E. Preparation of Structurally Well-Defined PolymerNanoparticle Hybrids with Controlled/Living Radical Polymerizations. Journal of the American Chemical Society 1999, 121 (32), 7409-7410.

(139) von Werne, T.; Patten, T. E. Atom Transfer Radical Polymerization from Nanoparticles: A Tool for the Preparation of Well-Defined Hybrid Nanostructures and for Understanding the Chemistry of Controlled/"living" Radical Polymerizations from Surfaces. Journal of the American Chemical Society 2001, 123 (31), 7497-7505.

(140) Pyun, J.; Jia, S.; Kowalewski, T.; Patterson, G. D.; Matyjaszewski, K. Synthesis and Characterization of Organic/Inorganic Hybrid Nanoparticles: Kinetics of Surface-Initiated Atom Transfer Radical Polymerization and Morphology of Hybrid Nanoparticle Ultrathin Films. Macromolecules 2003, 36 (14), 5094-5104.

(141) Lei, Z.; Bi, S. Preparation of Polymer Nanocomposites of Core-Shell Structure via Surface-Initiated Atom Transfer Radical Polymerizations. Materials Letters 2007, 61 (16), 3531-3534.

(142) Perruchot, C.; Khan, M. A.; Kamitsi, A.; Armes, S. P.; Von Werne, T.; Patten, T. E. Synthesis of Well-Defined, Polymer-Grafted Silica Particles by Aqueous ATRP. Langmuir 2001, 17 (15), 4479-4481.

(143) Popelensky, V. M.; Dorofeev, S. G.; Kononov, N. N.; Bubenov, S. S.; Vinokurov, A. A. Room Temperature Oxidation of Si Nanocrystals at Dry and Wet Air. Journal of Nanoparticle Research 2020, 22 (3), 54.

(144) Lawes, S.; Sun, Q.; Lushington, A.; Xiao, B.; Liu, Y.; Sun, X. Inkjet-Printed Silicon as High Performance Anodes for Li-Ion Batteries. Nano Energy 2017, 36, 313-321.

(145) Drahi, E.; Blayac, S.; Borbely, A.; Benaben, P. Impact of Ink Synthesis on Processing of Inkjet-Printed Silicon Nanoparticle Thin Films: A Comparison of Rapid Thermal Annealing and Photonic Sintering. Thin Solid Films 2015, 574, 169-176.

(146) Drahi, E.; Gupta, A.; Blayac, S.; Saunier, S.; Benaben, P. Characterization of Sintered Inkjet-Printed Silicon Nanoparticle Thin Films for Thermoelectric Devices. Physica Status Solidi (A) Applications and Materials Science 2014, 211 
(6), 1301-1307.

(147) Gupta, A.; Khalil, A. S. G.; Offer, M.; Geller, M.; Winterer, M.; Lorke, A.; Wiggers, H. Synthesis and Ink-Jet Printing of Highly Luminescing Silicon Nanoparticles for Printable Electronics. Journal of Nanoscience and Nanotechnology 2011, 11 (6), 5028-5033.

(148) Strebhardt, K.; Ullrich, A. Paul Ehrlich's Magic Bullet Concept: 100 Years of Progress. Nature Reviews Cancer 2008, 8 (6), 473-480.

(149) Valent, P.; Groner, B.; Schumacher, U.; Superti-Furga, G.; Busslinger, M.; Kralovics, R.; Zielinski, C.; Penninger, J. M.; Kerjaschki, D.; Stingl, G.; Smolen, J. S.; Valenta, R.; Lassmann, H.; Kovar, H.; Jäger, U.; Kornek, G.; Müller, M.; Sörgel, F. Paul Ehrlich (1854-1915) and His Contributions to the Foundation and Birth of Translational Medicine. Journal of Innate Immunity 2016, 8 (2), 111-120.

(150) Panyam, J.; Labhasetwar, V. Biodegradable Nanoparticles for Drug and Gene Delivery to Cells and Tissue. Advanced Drug Delivery Reviews 2003, 55 (3), 329 347.

(151) Algar, W. R.; Prasuhn, D. E.; Stewart, M. H.; Jennings, T. L.; Blanco-Canosa, J. B.; Dawson, P. E.; Medintz, I. L. The Controlled Display of Biomolecules on Nanoparticles: A Challenge Suited to Bioorthogonal Chemistry. Bioconjugate Chemistry 2011, 22 (5), 825-858.

(152) Xie, J.; Lee, S.; Chen, X. Nanoparticle-Based Theranostic Agents. Advanced Drug Delivery Reviews 2010, 62 (11), 1064-1079.

(153) Rai, P.; Mallidi, S.; Zheng, X.; Rahmanzadeh, R.; Mir, Y.; Elrington, S.; Khurshid, A.; Hasan, T. Development and Applications of Photo-Triggered Theranostic Agents. Advanced Drug Delivery Reviews 2010, 62 (11), 1094-1124.

(154) Bayliss, S. C.; Heald, R.; Fletcher, D. I.; Buckberry, L. D. Culture of Mammalian Cells on Nanostructured Silicon. Advanced Materials 1999, 11 (4), 318-321.

(155) Park, J. H.; Gu, L.; Von Maltzahn, G.; Ruoslahti, E.; Bhatia, S. N.; Sailor, M. J. Biodegradable Luminescent Porous Silicon Nanoparticles for in Vivo Applications. Nature Materials 2009, 8 (4), 331-336.

(156) Cheng, X.; Lowe, S. B.; Reece, P. J.; Gooding, J. J. Colloidal Silicon Quantum Dots: From Preparation to the Modification of Self-Assembled Monolayers (SAMs) for Bio-Applications. Chemical Society Reviews 2014, 43 (8), 2680 2700.

(157) Chiappini, C.; De Rosa, E.; Martinez, J. O.; Liu, X.; Steele, J.; Stevens, M. M.; Tasciotti, E. Biodegradable Silicon Nanoneedles Delivering Nucleic Acids Intracellularly Induce Localized in Vivo Neovascularization. Nature Materials 2015, 14 (5), 532-539.

(158) Baati, T.; Al-Kattan, A.; Esteve, M. A.; Njim, L.; Ryabchikov, Y.; Chaspoul, F.; Hammami, M.; Sentis, M.; Kabashin, A. V.; Braguer, D. Ultrapure LaserSynthesized Si-Based Nanomaterials for Biomedical Applications: In Vivo Assessment of Safety and Biodistribution. Scientific Reports 2016, 6, 25400.

(159) Croissant, J. G.; Fatieiev, Y.; Khashab, N. M. Degradability and Clearance of Silicon, Organosilica, Silsesquioxane, Silica Mixed Oxide, and Mesoporous Silica Nanoparticles. Advanced Materials 2017, 29 (9), 10.1002/adma.201604634.

(160) Li, W.; Liu, Z.; Fontana, F.; Ding, Y.; Liu, D.; Hirvonen, J. T.; Santos, H. A. Tailoring Porous Silicon for Biomedical Applications: From Drug Delivery to 
Cancer Immunotherapy. Advanced Materials 2018, $30 \quad$ (24), 10.1002/adma.201703740.

(161) Santos, H. A. (2014). Porous Silicon for Biomedical Applications. Woodhead Publishing.

(162) Salonen, J.; Kaukonen, A. M.; Hirvonen, J.; Lehto, V. P. Mesoporous Silicon in Drug Delivery Applications. Journal of Pharmaceutical Sciences 2008, 97 (2), 632-653.

(163) Haidary, S. M.; Córcoles, E. P.; Ali, N. K. Nanoporous Silicon as Drug Delivery Systems for Cancer Therapies. Journal of Nanomaterials 2012, 2012, 830503.

(164) Santos, H. A.; Mäkilä, E.; Airaksinen, A. J.; Bimbo, L. M.; Hirvonen, J. Porous Silicon Nanoparticles for Nanomedicine: Preparation and Biomedical Applications. Nanomedicine 2014, 9 (4), 535-554.

(165) Venuta, A.; Wolfram, J.; Shen, H.; Ferrari, M. Post-Nano Strategies for Drug Delivery: Multistage Porous Silicon Microvectors. Journal of Materials Chemistry B 2017, 5 (2), 207-219.

(166) Savage, D. J.; Liu, X.; Curley, S. A.; Ferrari, M.; Serda, R. E. Porous Silicon Advances in Drug Delivery and Immunotherapy. Current Opinion in Pharmacology 2013, 13 (5), 834-841.

(167) Tasciotti, E.; Liu, X.; Bhavane, R.; Plant, K.; Leonard, A. D.; Price, B. K.; Cheng, M. M. C.; Decuzzi, P.; Tour, J. M.; Robertson, F.; Ferrari, M. Mesoporous Silicon Particles as a Multistage Delivery System for Imaging and Therapeutic Applications. Nature Nanotechnology 2008, 3 (3), 151-157.

(168) Park, J. H.; Gu, L.; von Maltzahn, G.; Ruoslahti, E.; Bhatia, S. N.; Sailor, M. J. Biodegradable Luminescent Porous Silicon Nanoparticles for in Vivo Applications. Nature Materials 2009, 8 (4), 331-336.

(169) Sviridov, A. P.; Andreev, V. G.; Ivanova, E. M.; Osminkina, L. A.; Tamarov, K. P.; Timoshenko, V. Y. Porous Silicon Nanoparticles as Sensitizers for Ultrasonic Hyperthermia. Applied Physics Letters 2013, 103 (19), 193110.

(170) Sviridov, A.; Tamarov, K.; Fesenko, I.; Xu, W.; Andreev, V.; Timoshenko, V.; Lehto, V. P. Cavitation Induced by Janus-like Mesoporous Silicon Nanoparticles Enhances Ultrasound Hyperthermia. Frontiers in Chemistry 2019, 7, 393.

(171) Anglin, E. J.; Cheng, L.; Freeman, W. R.; Sailor, M. J. Porous Silicon in Drug Delivery Devices and Materials. Advanced Drug Delivery Reviews 2008, 60 (11), 1266-1277.

(172) Hoang Thi, T. T.; Cao, V. du; Nguyen, T. N. Q.; Hoang, D. T.; Ngo, V. C.; Nguyen, D. H. Functionalized Mesoporous Silica Nanoparticles and Biomedical Applications. Materials Science and Engineering C 2019, 99, 631-656.

(173) Chen, W.; Glackin, C. A.; Horwitz, M. A.; Zink, J. I. Nanomachines and Other Caps on Mesoporous Silica Nanoparticles for Drug Delivery. Accounts of Chemical Research 2019, 52 (6), 1531-1542.

(174) Jung, Y.; Huh, Y.; Kim, D. Recent Advances in Surface Engineering of Porous Silicon Nanomaterials for Biomedical Applications. Microporous and Mesoporous Materials 2021, 310, 110673.

(175) Jakobsson, U.; Mäkilä, E.; Rahikkala, A.; Imlimthan, S.; Lampuoti, J.; Ranjan, S.; Heino, J.; Jalkanen, P.; Köster, U.; Mizohata, K.; Santos, H. A.; Salonen, J.; Airaksinen, A. J.; Sarparanta, M.; Helariutta, K. Preparation and in Vivo 
Evaluation of Red Blood Cell Membrane Coated Porous Silicon Nanoparticles Implanted with 155Tb. Nuclear Medicine and Biology 2020, 84-85, 102-110.

(176) Rahikkala, A.; Fontana, F.; Bauleth-Ramos, T.; Correia, A.; Kemell, M.; Seitsonen, J.; Mäkilä, E.; Sarmento, B.; Salonen, J.; Ruokolainen, J.; Hirvonen, J.; Santos, H. A. Hybrid Red Blood Cell Membrane Coated Porous Silicon Nanoparticles Functionalized with Cancer Antigen Induce Depletion of T Cells. RSC Advances 2020, 10 (58), 35198-35205.

(177) Zhang, H.; Cui, W.; Qu, X.; Wu, H.; Qu, L.; Zhang, X.; Mäkilä, E.; Salonen, J.; Zhu, Y.; Yang, Z.; Chen, D.; Santos, H. A.; Hai, M.; Weitz, D. A. PhotothermalResponsive Nanosized Hybrid Polymersome as Versatile Therapeutics Codelivery Nanovehicle for Effective Tumor Suppression. Proceedings of the National Academy of Sciences of the United States of America 2019, 116 (16), 7744-7749.

(178) Wang, C. F.; Sarparanta, M. P.; Mäkilä, E. M.; Hyvönen, M. L. K.; Laakkonen, P. M.; Salonen, J. J.; Hirvonen, J. T.; Airaksinen, A. J.; Santos, H. A. Multifunctional Porous Silicon Nanoparticles for Cancer Theranostics. Biomaterials 2015, 48, 108-118.

(179) Kumeria, T.; McInnes, S. J. P.; Maher, S.; Santos, A. Porous Silicon for Drug Delivery Applications and Theranostics: Recent Advances, Critical Review and Perspectives. Expert Opinion on Drug Delivery 2017, 14 (12), 1407-1422.

(180) Airaksinen, A. (2014). Theranostic Imaging with Porous Silicon. In L. Canham (Ed.). Handbook of Porous Silicon (pp 1-12). Springer International Publishing.

(181) Ye, H. L.; Cai, S. J.; Li, S.; He, X. W.; Li, W. Y.; Li, Y. H.; Zhang, Y. K. OnePot Microwave Synthesis of Water-Dispersible, High Fluorescence Silicon Nanoparticles and Their Imaging Applications in Vitro and in Vivo. Analytical Chemistry 2016, 88 (23), 11631-11638.

(182) Tu, C.; Ma, X.; Pantazis, P.; Kauzlarich, S. M.; Louie, A. Y. Paramagnetic, Silicon Quantum Dots for Magnetic Resonance and Two-Photon Imaging of Macrophages. Journal of the American Chemical Society 2010, 132 (6), 20162023.

(183) Dou, Y. K.; Shang, Y.; He, X. W.; Li, W. Y.; Li, Y. H.; Zhang, Y. K. Preparation of a Ruthenium-Complex-Functionalized Two-Photon-Excited Red Fluorescence Silicon Nanoparticle Composite for Targeted Fluorescence Imaging and Photodynamic Therapy in Vitro. ACS Applied Materials and Interfaces 2019, 11 (15), 13954-13963.

(184) Kharin, A. Y.; Lysenko, V. V.; Rogov, A.; Ryabchikov, Y. V.; Geloen, A.; Tishchenko, I.; Marty, O.; Sennikov, P. G.; Kornev, R. A.; Zavestovskaya, I. N.; Kabashin, A. V.; Timoshenko, V. Y. Bi-Modal Nonlinear Optical Contrast from Si Nanoparticles for Cancer Theranostics. Advanced Optical Materials 2019, 7 (13), 1801728.

(185) Brown, S. Photodynamic Therapy: Two Photons Are Better than One. Nature Photonics 2008, 2 (7), 394-395.

(186) Shen, Y.; Shuhendler, A. J.; Ye, D.; Xu, J. J.; Chen, H. Y. Two-Photon Excitation Nanoparticles for Photodynamic Therapy. Chemical Society Reviews 2016, 45 (24), 6725-6741.

(187) Zhou, J.; Yang, Y.; Zhang, C. Y. Toward Biocompatible Semiconductor Quantum 
Dots: From Biosynthesis and Bioconjugation to Biomedical Application. Chemical Reviews 2015, 115 (21), 11669-11717.

(188) Atkins, T. M.; Thibert, A.; Larsen, D. S.; Dey, S.; Browning, N. D.; Kauzlarich, S. M. Femtosecond Ligand/Core Dynamics of Microwave-Assisted Synthesized Silicon Quantum Dots in Aqueous Solution. Journal of the American Chemical Society 2011, 133 (51), 20664-20667.

(189) Fojtik, A.; Giersig, M.; Henglein, A. Formation of Nanometer-Size Silicon Particles in a Laser Induced Plasma in SiH4. Berichte der Bunsengesellschaft für physikalische Chemie 1993, 97 (11), 1493-1496.

(190) Liu, X.; Zhang, Y.; Yu, T.; Qiao, X.; Gresback, R.; Pi, X.; Yang, D. Optimum Quantum Yield of the Light Emission from 2 to $10 \mathrm{Nm}$ Hydrosilylated Silicon Quantum Dots. Particle and Particle Systems Characterization 2016, 33 (1), 44 52.

(191) Zhong, Y.; Peng, F.; Bao, F.; Wang, S.; Ji, X.; Yang, L.; Su, Y.; Lee, S. T.; He, Y. Large-Scale Aqueous Synthesis of Fluorescent and Biocompatible Silicon Nanoparticles and Their Use as Highly Photostable Biological Probes. Journal of the American Chemical Society 2013, 135 (22), 8350-8356.

(192) Zhong, Y.; Sun, X.; Wang, S.; Peng, F.; Bao, F.; Su, Y.; Li, Y.; Lee, S. T.; He, Y. Facile, Large-Quantity Synthesis of Stable, Tunable-Color Silicon Nanoparticles and Their Application for Long-Term Cellular Imaging. ACS Nano 2015, 9 (6), 5958-5967.

(193) Pi, X. D.; Liptak, R. W.; Deneen Nowak, J.; Wells, N. P.; Carter, C. B.; Campbell, S. A.; Kortshagen, U. Air-Stable Full-Visible-Spectrum Emission from Silicon Nanocrystals Synthesized by an All-Gas-Phase Plasma Approach. Nanotechnology 2008, 19 (24), 245603.

(194) Cao, Z.; Peng, F.; Hu, Z.; Chu, B.; Zhong, Y.; Su, Y.; He, S.; He, Y. In Vitro Cellular Behaviors and Toxicity Assays of Small-Sized Fluorescent Silicon Nanoparticles. Nanoscale 2017, 9 (22), 7602-7611.

(195) Ji, X.; Peng, F.; Zhong, Y.; Su, Y.; Jiang, X.; Song, C.; Yang, L.; Chu, B.; Lee, S. T.; He, Y. Highly Fluorescent, Photostable, and Ultrasmall Silicon Drug Nanocarriers for Long-Term Tumor Cell Tracking and in-Vivo Cancer Therapy. Advanced Materials 2015, 27 (6), 1029-1034.

(196) Tang, M.; Ji, X.; Xu, H.; Zhang, L.; Jiang, A.; Song, B.; Su, Y.; He, Y. Photostable and Biocompatible Fluorescent Silicon Nanoparticles-Based Theranostic Probes for Simultaneous Imaging and Treatment of Ocular Neovascularization. Analytical Chemistry 2018, 90 (13), 8188-8195.

(197) Zhou, Y.; Zhang, Y.; Zhong, Y.; Fu, R.; Wu, S.; Wang, Q.; Wang, H.; Su, Y.; Zhang, H.; He, Y. The in Vivo Targeted Molecular Imaging of Fluorescent Silicon Nanoparticles in Caenorhabditis Elegans. Nano Research 2018, 11 (5), 23362346.

(198) Lai, C. H.; Hütter, J.; Hsu, C. W.; Tanaka, H.; Varela-Aramburu, S.; De Cola, L.; Lepenies, B.; Seeberger, P. H. Analysis of Carbohydrate-Carbohydrate Interactions Using Sugar-Functionalized Silicon Nanoparticles for Cell Imaging. Nano Letters 2016, 16 (1), 807-811.

(199) Pang, J.; Su, Y.; Zhong, Y.; Peng, F.; Song, B.; He, Y. Fluorescent Silicon Nanoparticle-Based Gene Carriers Featuring Strong Photostability and Feeble 
Cytotoxicity. Nano Research 2016, 9 (10), 3027-3037.

(200) Zhang, L.; Ji, X.; Su, Y.; Zhai, X.; Xu, H.; Song, B.; Jiang, A.; Guo, D.; He, Y. Fluorescent Silicon Nanoparticles-Based Nanotheranostic Agents for Rapid Diagnosis and Treatment of Bacteria-Induced Keratitis. Nano Research 2021, 14 (1), 52-58.

(201) Xiao, L.; Gu, L.; Howell, S. B.; Sailor, M. J. Porous Silicon Nanoparticle Photosensitizers for Singlet Oxygen and Their Phototoxicity against Cancer Cells. ACS Nano 2011, 5 (5), 3651-3659.

(202) Kovalev, D.; Gross, E.; Künzner, N.; Koch, F.; Timoshenko, V. Y.; Fujii, M. Resonant Electronic Energy Transfer from Excitons Confined in Silicon Nanocrystals to Oxygen Molecules. Physical Review Letters 2002, 89 (13), 1374011-1374014.

(203) Osminkina, L. A.; Tamarov, K. P.; Sviridov, A. P.; Galkin, R. A.; Gongalsky, M. B.; Solovyev, V. v.; Kudryavtsev, A. A.; Timoshenko, V. Y. Photoluminescent Biocompatible Silicon Nanoparticles for Cancer Theranostic Applications. Journal of Biophotonics 2012, 5 (7), 529-535.

(204) Al-Kattan, A.; Ali, L. M. A.; Daurat, M.; Mattana, E.; Gary-Bobo, M. Biological Assessment of Laser-Synthesized Silicon Nanoparticles Effect in Two-Photon Photodynamic Therapy on Breast Cancer Mcf-7 Cells. Nanomaterials 2020, 10 (8), 1-11.

(205) DeRosa, M. C.; Crutchley, R. J. Photosensitized Singlet Oxygen and Its Applications. Coordination Chemistry Reviews 2002, 233-234, 351-371.

(206) Lee, C.; Kim, H.; Hong, C.; Kim, M.; Hong, S. S.; Lee, D. H.; Lee, W. I. Porous Silicon as an Agent for Cancer Thermotherapy Based on Near-Infrared Light Irradiation. Journal of Materials Chemistry 2008, 18 (40), 4790-4795.

(207) Hong, C.; Lee, J.; Zheng, H.; Hong, S. S.; Lee, C. Porous Silicon Nanoparticles for Cancer Photothermotherapy. Nanoscale Research Letters 2011, 6 (1), 321.

(208) Gongalsky, M.; Gvindzhiliia, G.; Tamarov, K.; Shalygina, O.; Pavlikov, A.; Solovyev, V.; Kudryavtsev, A.; Sivakov, V.; Osminkina, L. A. Radiofrequency Hyperthermia of Cancer Cells Enhanced by Silicic Acid Ions Released during the Biodegradation of Porous Silicon Nanowires. ACS Omega 2019, 4 (6), 1066210669.

(209) Tamarov, K. P.; Osminkina, L. A.; Zinovyev, S. v.; Maximova, K. A.; Kargina, J. v.; Gongalsky, M. B.; Ryabchikov, Y.; Al-Kattan, A.; Sviridov, A. P.; Sentis, M.; Ivanov, A. v.; Nikiforov, V. N.; Kabashin, A. v.; Timoshenko, V. Y. Radio Frequency Radiation-Induced Hyperthermia Using Si Nanoparticle-Based Sensitizers for Mild Cancer Therapy. Scientific Reports 2014, 4, 7034.

(210) Nikolaou, P.; Goodson, B. M.; Chekmenev, E. Y. NMR Hyperpolarization Techniques for Biomedicine. Chemistry - A European Journal 2015, 21 (8), 31563166.

(211) Halse, M. E. Perspectives for Hyperpolarisation in Compact NMR. TrAC - Trends in Analytical Chemistry 2016, 83, 76-83.

(212) Kwiatkowski, G.; Jähnig, F.; Steinhauser, J.; Wespi, P.; Ernst, M.; Kozerke, S. Nanometer Size Silicon Particles for Hyperpolarized MRI. Scientific Reports 2017, 7 (1), 7946.

(213) Keshari, K. R.; Wilson, D. M. Chemistry and Biochemistry of ${ }^{13}$ C Hyperpolarized 
Magnetic Resonance Using Dynamic Nuclear Polarization. Chem Soc Rew 2014, 43 (5), 1627-1659.

(214) Seo, H.; Choi, I.; Whiting, N.; Hu, J.; Luu, Q. S.; Pudakalakatti, S.; McCowan, C.; Kim, Y.; Zacharias, N.; Lee, S.; Bhattacharya, P.; Lee, Y. Hyperpolarized Porous Silicon Nanoparticles: Potential Theragnostic Material for ${ }^{29} \mathrm{Si}$ Magnetic Resonance Imaging. ChemPhysChem 2018, 19 (17), 2143-2147.

(215) Shulman, R. G.; Wyluda, B. J. Nuclear Magnetic Resonance of Si29 in N- and pType Silicon. Physical Review 1956, 103 (4), 1127-1129.

(216) Wang, Z. J.; Ohliger, M. A.; Larson, P. E. Z.; Gordon, J. W.; Bok, R. A.; Slater, J.; Villanueva-Meyer, J. E.; Hess, C. P.; Kurhanewicz, J.; Vigneron, D. B. Hyperpolarized ${ }^{13} \mathrm{C}$ MRI: State of the Art and Future Directions. Radiology 2019, 291 (2), 273-284.

(217) Aptekar, J. W.; Cassidy, M. C.; Johnson, A. C.; Barton, R. A.; Lee, M.; Ogier, A. C.; Vo, C.; Anahtar, M. N.; Ren, Y.; Bhatia, S. N.; Ramanathan, C.; Cory, D. G.; Hill, A. L.; Mair, R. W.; Rosen, M. S.; Walsworth, R. L.; Marcus, C. M. Silicon Nanoparticles as Hyperpolarized Magnetic Resonance Imaging Agents. ACS Nano 2009, 3 (12), 4003-4008.

(218) Atkins, T. M.; Cassidy, M. C.; Lee, M.; Ganguly, S.; Marcus, C. M.; Kauzlarich, S. M. Synthesis of Long T 1 Silicon Nanoparticles for Hyperpolarized ${ }^{29} \mathrm{Si}$ Magnetic Resonance Imaging. ACS Nano 2013, 7 (2), 1609-1617.

(219) Whiting, N.; Hu, J.; Shah, J. V.; Cassidy, M. C.; Cressman, E.; Millward, N. Z.; Menter, D. G.; Marcus, C. M.; Bhattacharya, P. K. Real-Time MRI-Guided Catheter Tracking Using Hyperpolarized Silicon Particles. Scientific Reports 2015, 5, 12842.

(220) Whiting, N.; Hu, J.; Zacharias, N. M.; Lokesh, G. L. R.; Volk, D. E.; Menter, D. G.; Rupaimoole, R.; Previs, R.; Sood, A. K.; Bhattacharya, P. Developing Hyperpolarized Silicon Particles for in Vivo MRI Targeting of Ovarian Cancer . Journal of Medical Imaging 2016, 3 (3), 036001.

(221) Singh, D.; Dilnawaz, F.; Sahoo, S. K. Challenges of Moving Theranostic Nanomedicine into the Clinic. Nanomedicine 2020, 15 (2), 111-114.

(222) Devi, M. G.; Balachandran, S. A Review on Synthesis, Characterization and Applications of Silica Particles. International Journal of Advanced Engineering Research and Technology (IJAERT) 2016, 4 (7), 249-254.

(223) Fu, S.-Y.; Feng, X.-Q.; Lauke, B.; Mai, Y.-W. Effects of Particle Size, Particle/Matrix Interface Adhesion and Particle Loading on Mechanical Properties of Particulate-Polymer Composites. Composites Part B: Engineering 2008, 39 (6), 933-961.

(224) Fu, S.; Sun, Z.; Huang, P.; Li, Y.; Hu, N. Some Basic Aspects of Polymer Nanocomposites: A Critical Review. Nano Materials Science 2019, 1 (1), 2-30.

(225) Hanemann, T.; Szabó, D. V. Polymer-Nanoparticle Composites: From Synthesis to Modern Applications. Materials 2010, 3 (6), 3468-3517.

(226) Puretskiy, N.; Ionov, L. Synthesis of Robust Raspberry-like Particles Using Polymer Brushes. Langmuir 2011, 27 (6), 3006-3011.

(227) Ming, W.; Wu, D.; Van Benthem, R.; De With, G. Superhydrophobic Films from Raspberry-like Particles. Nano Letters 2005, 5 (11), 2298-2301.

(228) Ebert, D.; Bhushan, B. Durable Lotus-Effect Surfaces with Hierarchical Structure 
Using Micro- and Nanosized Hydrophobic Silica Particles. Journal of Colloid and Interface Science 2012, 368 (1), 584-591.

(229) Li, X. M.; Reinhoudt, D.; Crego-Calama, M. What Do We Need for a Superhydrophobic Surface? A Review on the Recent Progress in the Preparation of Superhydrophobic Surfaces. Chemical Society Reviews 2007, 36 (8), 13501368.

(230) Subhash Latthe, S.; Basavraj Gurav, A.; Shridhar Maruti, C.; Shrikant Vhatkar, R. Recent Progress in Preparation of Superhydrophobic Surfaces: A Review. Journal of Surface Engineered Materials and Advanced Technology 2012, 02 (02), 76-94.

(231) Hu, C.; West, K. R.; Scherman, O. A. Hollow Mesoporous Raspberry-like Colloids with Removable Caps as Photoresponsive Nanocontainers. Nanoscale 2016, 8 (15), 7840-7844.

(232) Li, D.; Zhu, Y.; Mao, C. One-Pot Synthesis of Surface Roughness Controlled Hollow Silica Spheres with Enhanced Drug Loading and Release Profiles under Ambient Conditions in Aqueous Solutions. Journal of Materials Chemistry B 2013, 1 (40), 5515-5520.

(233) Liu, S.; Latthe, S. S.; Yang, H.; Liu, B.; Xing, R. Raspberry-like Superhydrophobic Silica Coatings with Self-Cleaning Properties. Ceramics International 2015, 41 (9), 11719-11725.

(234) Blanckenberg, A.; Malgas-Enus, R. Raspberry-like Gold-Decorated Silica (SSxAMPS-Au) Nanoparticles for the Reductive Discoloration of Dyes. SN Applied Sciences 2019, 1 (7), 787.

(235) Chen, Z.; Xu, J.; Xiang, X.; Ren, D.; Chen, N.; Ye, Z.; Li, X.; Ma, S.; Chen, Q. Facile One-Step Dialysis Strategy for Preparation of Porous Silica Nanoparticles with Rough Surface. Nano 2020, 15 (3), 7-14.

(236) Blander, M.; Katz, J. L. Bubble Nucleation in Liquids. AIChE Journal 1975, 21 (5), 833-848.

(237) Mukai, K. (2005). Interfacial Phenomena, Metals Processing and Properties. In S. Seetharaman (Ed.). Fundamentals of Metallurgy (pp 237-269). Woodhead Publishing.

(238) Volmer, M. Über Keimbildung Und Keimwirkung Als Spezialfälle Der Heterogenen Katalyse. Zeitschrift für Elektrochemie 1929, 35 (9), 555-561.

(239) Fletcher, N. H. Size Effect in Heterogeneous Nucleation. The Journal of Chemical Physics 1958, 29 (3), 572-576.

(240) Liu, Q.; Zhu, Y.; Yang, G.; Yang, Q. Nucleation Thermodynamics inside Micro/Nanocavity. Journal of Materials Science and Technology 2008, 24 (2), $183-186$.

(241) Costeux, S. CO 2 -Blown Nanocellular Foams. Journal of Applied Polymer Science 2014, 131 (23), 10.1002/app.41293.

(242) Liu, S.; Duvigneau, J.; Vancso, G. J. Nanocellular Polymer Foams as Promising High Performance Thermal Insulation Materials. European Polymer Journal 2015, 65, 33-45.

(243) Jacobs, L. J. M.; Kemmere, M. F.; Keurentjes, J. T. F. Sustainable Polymer Foaming Using High Pressure Carbon Dioxide: A Review on Fundamentals, Processes and Applications. Green Chemistry 2008, 10 (7), 731-773.

(244) Sauceau, M.; Fages, J.; Common, A.; Nikitine, C.; Rodier, E. New Challenges in 
Polymer Foaming: A Review of Extrusion Processes Assisted by Supercritical Carbon Dioxide. Progress in Polymer Science (Oxford) 2011, 36 (6), 749-766.

(245) Lee, L. J.; Zeng, C.; Cao, X.; Han, X.; Shen, J.; Xu, G. Polymer Nanocomposite Foams. Composites Science and Technology 2005, 65 (15-16 SPEC. ISS.), 23442363.

(246) Liu, S. (2017). Designer Nanoparticles as Efficient Nucleation Agents in Polymer Foaming. University of Twente. https://doi.org/10.3990/1.9789036544023

(247) Colton, J. S.; Suh, N. P. The Nucleation of Microcellular Thermoplastic Foam with Additives: Part I: Theoretical Considerations. Polymer Engineering \& Science 1987, 27 (7), 485-492.

(248) Colton, J. S.; Suh, N. P. Nucleation of Microcellular Foam: Theory and Practice. Polymer Engineering \& Science 1987, 27 (7), 500-503.

(249) Colton, J. S.; Suh, N. P. The Nucleation of Microcellular Thermoplastic Foam with Additives: Part II: Experimental Results and Discussion. Polymer Engineering \& Science 1987, 27 (7), 493-499.

(250) Colton, J. S.; Suh, N. P. Nucleation of Microcellular Foam: Theory and Practice. Polymer Engineering \& Science 1987, 27 (7), 500-503.

(251) Colton, J. S.; Suh, N. P. The Nucleation of Microcellular Thermoplastic Foam with Additives: Part I: Theoretical Considerations. Polymer Engineering \& Science 1987, 27 (7), 485-492.

(252) Colton, J. S.; Suh, N. P. The Nucleation of Microcellular Thermoplastic Foam with Additives: Part II: Experimental Results and Discussion. Polymer Engineering \& Science 1987, 27 (7), 493-499.

(253) Zhai, W.; Yu, J.; Wu, L.; Ma, W.; He, J. Heterogeneous Nucleation Uniformizing Cell Size Distribution in Microcellular Nanocomposites Foams. Polymer 2006, 47 (21), 7580-7589.

(254) Velasco, J. I.; Antunes, M.; Ayyad, O.; López-Cuesta, J. M.; Gaudon, P.; SaizArroyo, C.; Rodríguez-Pérez, M. A.; de Saja, J. A. Foaming Behaviour and Cellular Structure of LDPE/Hectorite Nanocomposites. Polymer 2007, 48 (7), 2098-2108.

(255) Shen, J.; Zeng, C.; Lee, L. J. Synthesis of Polystyrene-Carbon Nanofibers Nanocomposite Foams. Polymer 2005, 46 (14), 5218-5224.

(256) Zeng, C.; Han, X.; Lee, L. J.; Koelling, K. W.; Tomasko, D. L. Polymer-Clay Nanocomposite Foams Prepared Using Carbon Dioxide. Advanced Materials 2003, 15 (20), 1743-1747.

(257) Famili, M. H. N.; Janani, H.; Enayati, M. S. Foaming of a Polymer-Nanoparticle System: Effect of the Particle Properties. Journal of Applied Polymer Science 2011, 119 (5), 2847-2856.

(258) Chen, L.; Ozisik, R.; Schadler, L. S. The Influence of Carbon Nanotube Aspect Ratio on the Foam Morphology of MWNT/PMMA Nanocomposite Foams. Polymer 2010, 51 (11), 2368-2375.

(259) Goren, K.; Chen, L.; Schadler, L. S.; Ozisik, R. Influence of Nanoparticle Surface Chemistry and Size on Supercritical Carbon Dioxide Processed Nanocomposite Foam Morphology. Journal of Supercritical Fluids 2010, 51 (3), 420-427.

(260) Urbanczyk, L.; Calberg, C.; Detrembleur, C.; Jérôme, C.; Alexandre, M. Batch Foaming of SAN/Clay Nanocomposites with $\mathrm{ScCO}_{2}$ : A Very Tunable Way of 
Controlling the Cellular Morphology. Polymer 2010, 51 (15), 3520-3531.

(261) Harikrishnan, G.; Patro, T. U.; Unni, A. R.; Khakhar, D. V. Clay Nanoplatelet Induced Morphological Evolutions during Polymeric Foaming. Soft Matter 2011, 7 (15), 6801-6804.

(262) Liu, S.; Eijkelenkamp, R.; Duvigneau, J.; Vancso, G. J. Silica-Assisted Nucleation of Polymer Foam Cells with Nanoscopic Dimensions: Impact of Particle Size, Line Tension, and Surface Functionality. ACS Applied Materials and Interfaces 2017, 9 (43), 37929-37940.

(263) Liu, S.; Yin, S.; Duvigneau, J.; Vancso, G. J. Bubble Seeding Nanocavities: Multiple Polymer Foam Cell Nucleation by Polydimethylsiloxane-Grafted Designer Silica Nanoparticles. ACS Nano 2020, 14 (2), 1623-1634.

(264) Vancso, Gyula J.; Duvigneau, Joost; Nederkoorn, P.H.J.; Wassing, T. Polymer Foam Comprising a Polymer and Nanoparticles, and Nanoparticles for the Manufacture of Such Foam. WO2014073960, 2014.

(265) Liu, S.; Zoetebier, B.; Hulsman, L.; Zhang, Y.; Duvigneau, J.; Vancso, G. J. Nanocellular Polymer Foams Nucleated by Core-Shell Nanoparticles. Polymer 2016, 104, 22-30.

(266) Liu, S.; Pandey, A.; Duvigneau, J.; Vancso, J.; Snoeijer, J. H. Size-Dependent Submerging of Nanoparticles in Polymer Melts: Effect of Line Tension. Macromolecules 2018, 51 (7), 2411-2417.

(267) Laidler, K. J. A Glossary of Terms Used in Chemical Kinetics, Including Reaction Dynamics (IUPAC Recommendations 1996). Pure and Applied Chemistry 1996, 68 (1), 149-192.

(268) Bkell.

Activation

energy https://commons.wikimedia.org/wiki/File:Activation_energy.svg (accessed 2021-09-06).

(269) Li, H. H.; Yu, S. H. Recent Advances on Controlled Synthesis and Engineering of Hollow Alloyed Nanotubes for Electrocatalysis. Advanced Materials 2019, 31 (38), 1803503.

(270) Wang, J. L.; Ando, R. A.; Camargo, P. H. C. Investigating the Plasmon-Mediated Catalytic Activity of Agau Nanoparticles as a Function of Composition: Are Two Metals Better than One? ACS Catalysis 2014, 4 (11), 3815-3819.

(271) Jia, C. J.; Schüth, F. Colloidal Metal Nanoparticles as a Component of Designed Catalyst. Physical Chemistry Chemical Physics 2011, 13 (7), 2457-2487.

(272) Liu, L.; Corma, A. Metal Catalysts for Heterogeneous Catalysis: From Single Atoms to Nanoclusters and Nanoparticles. Chemical Reviews 2018, 118 (10), 4981-5079.

(273) Song, H.; Rioux, R. M.; Hoefelmeyer, J. D.; Komor, R.; Niesz, K.; Grass, M.; Yang, P.; Somorjai, G. A. Hydrothermal Growth of Mesoporous SBA-15 Silica in the Presence of PVP-Stabilized Pt Nanoparticles: Synthesis, Characterization, and Catalytic Properties. Journal of the American Chemical Society 2006, 128 (9), 3027-3037.

(274) Banerjee, S.; Das, J.; Santra, S. Native Silica Nanoparticle Catalyzed AntiMarkovnikov Addition of Thiols to Inactivated Alkenes and Alkynes: A New Route to Linear and Vinyl Thioethers. Tetrahedron Letters 2009, 50 (1), 124-127.

(275) Banerjee, S.; Santra, S. Remarkable Catalytic Activity of Silica Nanoparticle in 
the Bis-Michael Addition of Active Methylene Compounds to Conjugated Alkenes. Tetrahedron Letters 2009, 50 (18), 2037-2040.

(276) Alvarez, S. G.; Hasegawa, S.; Hirano, M.; Komiya, S. Michael Reactions Promoted by H1-O-Enolatoruthenium(II) Complexes Derived from $\mathrm{Ru}(\mathrm{Cod})(\mathrm{Cot})$, Diphosphine, and Dimethyl Malonate. Tetrahedron Letters 1998, 39 (29), 5209-5212.

(277) Ranu, B. C.; Banerjee, S. Ionic Liquid as Catalyst and Reaction Medium. The Dramatic Influence of a Task-Specific Ionic Liquid, [BmIm] $\mathrm{OH}$, in Michael Addition of Active Methylene Compounds to Conjugated Ketones, Carboxylic Esters, and Nitriles. Organic Letters 2005, 7 (14), 3049-3052.

(278) Ranu, B. C.; Banerjee, S.; Jana, R. Ionic Liquid as Catalyst and Solvent: The Remarkable Effect of a Basic Ionic Liquid, [bmIm]OH on Michael Addition and Alkylation of Active Methylene Compounds. Tetrahedron 2007, 63 (3), 776-782.

(279) Kundu, S.; Roy, B.; Basu, B. Silica: An Efficient Catalyst for One-Pot Regioselective Synthesis of Dithioethers. Beilstein Journal of Organic Chemistry 2014, 10, 26-33.

(280) Nikoofar, K.; Khalili, Z. Nano-SiO 2 : A Heterogeneous and Reusable Catalyst for the One-Pot Synthesis of Symmetrical and Unsymmetrical 3,3-Di(Aryl)Indolin2-Ones under Solvent-Free Conditions. Zeitschrift fur Naturforschung - Section B Journal of Chemical Sciences 2016, 71 (1), 31-36.

(281) Ramazani, A.; Dastanra, K.; Zeinali Nasrabadi, F.; Karimi, Z.; Rouhani, M.; Hosseini, M. Silica Nanoparticles as a High Efficient Catalyst for the One-Pot Synthesis of 3-Oxo-3-Phenylpropanamid Derivatives from Isocyanides, Phenylacetaldehyde and Secondary Amines. Turkish Journal of Chemistry 2012, 36 (3), 467-476.

(282) Uemichi, Y.; Kashiwaya, Y.; Tsukidate, M.; Ayame, A.; Kanoh, H. Product Distribution in Degradation of Polypropylene over Silica-Alumina and $\mathrm{CaX}$ Zeolite Catalysts. Bulletin of the Chemical Society of Japan 1983, 56 (9), 27682773.

(283) Songip, A. R.; Masuda, T.; Kuwahara, H.; Hashimoto, K. Test to Screen Catalysts for Reforming Heavy Oil from Waste Plastics. Applied Catalysis B, Environmental 1993, 2 (2-3), 153-164.

(284) Ishihara, Y.; Nanbu, H.; Ikemura, T.; Takesue, T. Catalytic Decomposition of Polyethylene Using a Tubular Flow Reactor System. Fuel 1990, 69 (8), 978-984.

(285) Sakata, Y.; Uddin, M. A.; Muto, A. Degradation of Polyethylene and Polypropylene into Fuel Oil by Using Solid Acid and Non-Acid Catalysts. Journal of Analytical and Applied Pyrolysis 1999, 51 (1), 135-155.

(286) Ishihara, Y.; Nanbu, H.; Saido, K.; Ikemura, T.; Takesue, T. Mechanism for Gas Formation in Polyethylene Catalytic Decomposition. Polymer 1992, 33 (16), 3482-3486.

(287) Masuda, T.; Kuwahara, H.; Mukai, S. R.; Hashimoto, K. Production of High Quality Gasoline from Waste Polyethylene Derived Heavy Oil over Ni-REY Catalyst in Steam Atmosphere. Chemical Engineering Science 1999, 54 (13-14), 2773-2779.

(288) Aguado, J.; Serrano, D. P.; Escola, J. M.; Garagorri, E.; Fernández, J. A. Catalytic Conversion of Polyolefins into Fuels over Zeolite Beta. Polymer Degradation and 
Stability 2000, 69 (1), 11-16.

(289) Uemichi, Y.; Kashiwaya, Y.; Tsukidate, M.; Ayame, A.; Kanoh, H. Product Distribution in Degradation of Polypropylene over Silica-Alumina and CaX Zeolite Catalysts. Bulletin of the Chemical Society of Japan 1983, 56 (9), 27682773.

(290) Lee, K. H.; Noh, N. S.; Shin, D. H.; Seo, Y. Comparison of Plastic Types for Catalytic Degradation of Waste Plastics into Liquid Product with Spent FCC Catalyst. Polymer Degradation and Stability 2002, 78 (3), 539-544.

(291) De La Puente, G.; Arandes, J. M.; Sedran, U. A. Recycled Plastics in FCC Feedstocks: Specific Contributions. Industrial and Engineering Chemistry Research 1997, 36 (11), 4530-4534.

(292) Cardona, S. C.; Corma, A. Tertiary Recycling of Polypropylene by Catalytic Cracking in a Semibatch Stirred Reactor. Use of Spent Equilibrium FCC Commercial Catalyst. Applied Catalysis B: Environmental 2000, 25 (2-3), 151162.

(293) Uemichi, Y.; Makino, Y.; Kanazuka, T. Degradation of Polyethylene to Aromatic Hydrocarbons over Metal-Supported Activated Carbon Catalysts. Journal of Analytical and Applied Pyrolysis 1989, 14 (4), 331-344.

(294) Zhang, Z.; Hirose, T.; Nishio, S.; Morioka, Y.; Azuma, N.; Ueno, A.; Ohkita, H.; Okada, M. Chemical Recycling of Waste Polystyrene into Styrene over Solid Acids and Bases. Industrial and Engineering Chemistry Research 1995, 34 (12), 4514-4519.

(295) Sakata, Y.; Azhar Uddin, M.; Muto, A.; Kanada, Y.; Koizumi, K.; Murata, K. Catalytic Degradation of Polyethylene into Fuel Oil over Mesoporous Silica (KFS-16) Catalyst. Journal of Analytical and Applied Pyrolysis 1997, 43 (1), 1525.

(296) Inagaki, S.; Koiwai, A.; Suzuki, N.; Fukushima, Y.; Kuroda, K. Syntheses of Highly Ordered Mesoporous Materials, FSM-16, Derived from Kanemite. Bulletin of the Chemical Society of Japan 1996, 69 (5), 1449-1457. 


\section{Chapter}

\section{Dispersion Stability of Nonspherical Silicon Nanoparticles: Impact of Solvent Selection and Storage Time}

Long-term colloidal stability is of primary concern for numerous nanoparticle dispersion-based applications, such as inkjet printing. In order to assess printability of inks consisting of silicon nanoparticles (SiNPs) with complex agglomerated shapes in various alcohols and glycols, we studied the stability of the dispersions by several approaches. We utilized visual observations, optical turbidity profiling, analytical centrifugation (AC) and dynamic light scattering (for assessing hydrodynamically effective particle diameter values) to optimize colloidal stability under application relevant conditions. Predictions derived from Stokes' law and from AC results were compared to results by traditional (time-consuming) batch sedimentation experiments. We showed that although promising, neither the theoretical prediction using Stokes' law, nor accelerated measurements such as AC, capture particle behavior when considering particle agglomeration, backflow effects on the local particle concentration, and the possible long-term interactions between particles and solvents. For printing applications, long-term dispersion stability of SiNPs is very essential, which underlines the necessity of time-consuming methods for more complex particle geometries.

The contents of this Chapter will be published as: Yin, S.; Nguon, O. J.; Duvigneau, J.; Vancso, G. J. Dispersion Stability of Nonspherical Silicon Nanoparticles: Impact of Solvent Selection and Storage Time. Submitted 


\subsection{Introduction}

The materials chemistry field of colloidal nanoparticles covers an extensive and exciting area of nanotechnology, and therefore, colloidal nanoparticles have been extensively studied in the past few decades. ${ }^{1,2}$ This is mainly attributed to their unique and attractive properties compared to their bulk counterparts. On the one hand, colloidal nanoparticles can behave similarly to molecules in solution while possessing properties of bulk materials. On the other hand, when particles become small enough, quantum effects sometimes result in unexpected material properties. ${ }^{3}$ These properties give colloidal nanoparticles great potential in several application areas, such as biomedicine, catalysis, energy storage, solar cells, and light-emitting diodes (LEDs). ${ }^{4-6}$

Colloidal nanoparticle synthesis traces back to the 1950s when Turkevich reported the solution-based synthesis of gold nanoparticles. ${ }^{7}$ In the $1960 \mathrm{~s}$, Werner Stöber ${ }^{8}$ and NASA $^{9}$ reported the fabrication of colloidal silica and iron oxide suspensions, respectively. One of the first commercialization of colloidal nanoparticles was achieved in the 1980s when the cosmetics industry added zinc oxide and titanium oxide nanoparticles to sunscreen cream formulations. ${ }^{10}$ Currently, colloidal nanoparticles of various elements and compositions are readily prepared via solution-based bottom-up and solid-based top-down approaches., ${ }^{1,2,11}$ Additionally, colloidal nanoparticles are easily modified with different functional organic molecules or tethered polymers to either introduce new properties or enhance colloidal stability. ${ }^{12}$ For example, hydrosilylation has been widely used to introduce small organic molecules onto Silicon-containing nanoparticles. ${ }^{13}$

Colloidal Silicon-containing nanoparticles are an exciting category of nanoparticles. Compared to its bulk form, nanosilicon possesses several new and exciting properties, such as photoluminescence (PL), antimicrobial activity, and size-dependent electronic bandgaps. ${ }^{14}$ These interesting properties make Silicon-containing nanoparticles a promising candidate as ink components for printing electronic devices. ${ }^{15,16}$ One crucial parameter for formulating silicon nanoparticle-based inks is the long-term colloidal stability. ${ }^{17}$

Much effort by scientists and engineers worldwide has been applied to enhancing colloidal stability and better understanding nanoparticle sedimentation behavior. ${ }^{18-21}$ Several factors influence colloidal stability, such as nanoparticle concentration, surface charge, particle size, size distribution, surface chemistry, solvent, and solvent viscosity. ${ }^{21,22}$ Stokes' law is a popular theory that is widely applied to predict nanoparticle 
stability. ${ }^{23,24}$ It is commonly used to describe particle settling behavior in Newtonian fluids by simply monitoring the balance between the buoyant and gravitational forces. Stokes' law is only applicable to spherical particles and ignores the interaction between particles.

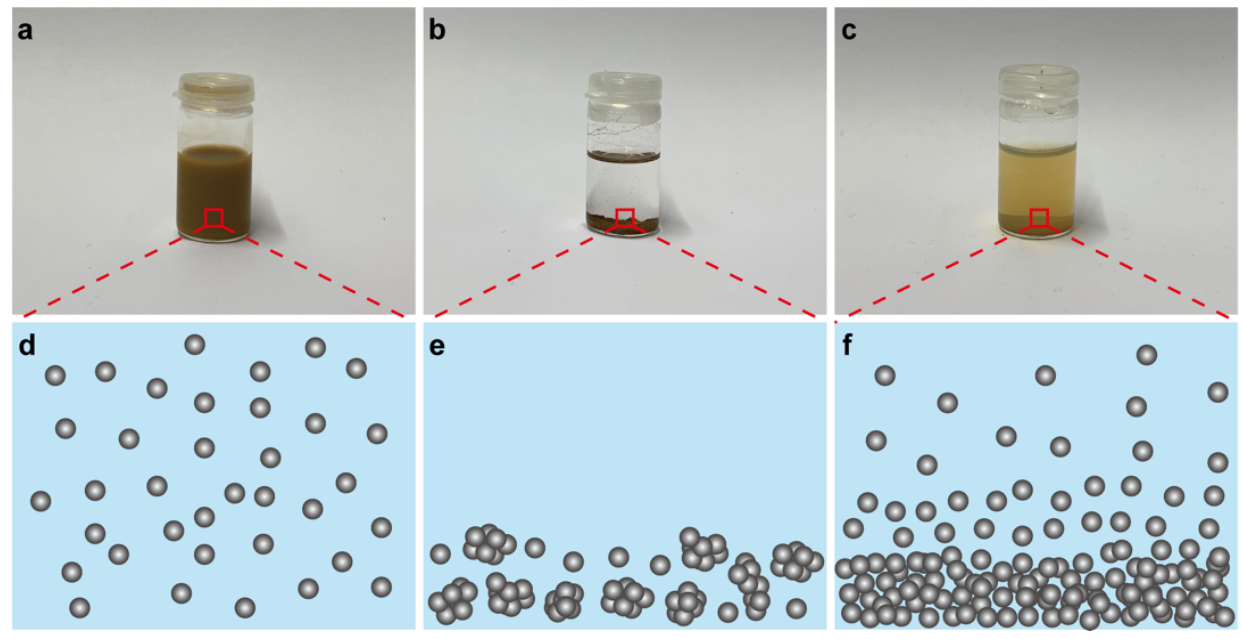

Figure 3.1 Images of silicon nanoparticles in (a) dispersed, (b) flocculated, and (c) sedimented states after suspension in ethanol, 1-hexane, and water, respectively, with a schematic drawing of the corresponding states (d-f).

Stable colloidal dispersions have great technological relevance in many application areas. $^{25-27}$ In this study, we focused on Si nanoparticles dispersed in various solvents to obtain practical, relevant information about stability windows; such information includes choice of solvent, the influence of external fields (such as gravitational), and time. The targeted application area is the inkjet printing of Si nanoparticles on various substrates to form stable, patterned Si films for subsequent processing into electrical circuits, such as RF security tags. Here, we focus exclusively on colloidal stability as a key enabling parameter for inkjet printing approaches. ${ }^{17}$

Due to the extensive number of factors, such as particle $\operatorname{size}^{28}$, shape, surface charge $^{29}$, and viscosity ${ }^{24}$, that can influence colloidal stability and the constraints of the theories on colloidal stability, it is difficult to predict nanoparticle stability and sedimentation behavior accurately ${ }^{30,31}$.

As a result, batch sedimentation testing is a commonly used method to address colloidal nanoparticle stability experimentally. ${ }^{32,33}$ In the batch sedimentation test, the settling behavior of particles with no influence other than the Earth's gravitational field is studied by storing the suspension in a vertical container. Several detection methods are 
available to follow particles' sedimentation behavior in dispersion, e.g., photo/video capturing, UV-Vis spectroscopy, dynamic light scattering (DLS), and transmittance electron microscopy. ${ }^{33,34}$ One practical difficulty related to colloidal stability testing in a simple gravitational field is related to the (sometimes extremely) long time needed to obtain a result.

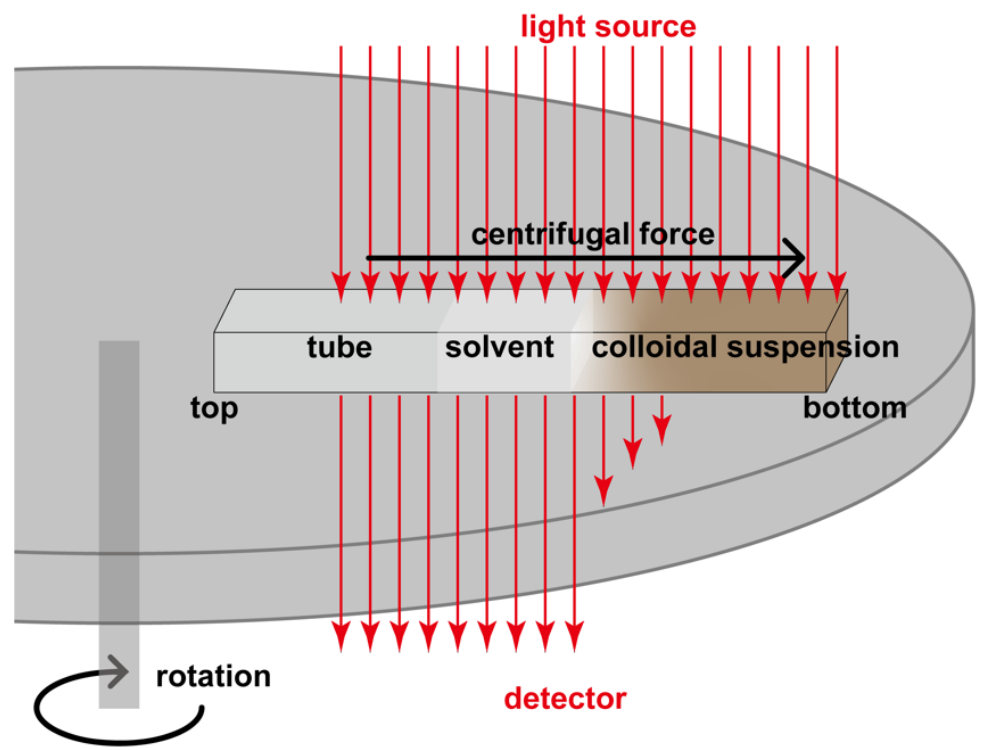

Figure 3.2 Schematic of analytical centrifugation. Colloidal suspensions in a transparent cuvette rotate at several thousand rpm to generate a centrifugal force. While turning, the cuvette passes through a light beam. A detector below the cuvette measures the transmitted light as a function of position within the cuvette at specified time intervals during centrifuge runs.

By applying a centrifugal field on colloidal suspensions, one can accelerate the sedimentation process and explore the effects of physical and chemical properties on nanoparticle colloidal stabilities. These methods can also help investigate the influence of additives, such as electrolytes and polymers, on colloidal stability. ${ }^{35}$ Analytical centrifugation (AC) (see Figure 3.2) is similar to the widely used analytical ultracentrifugation (AUC). Compared to AUC experiments, AC experiments typically run at a lower rotation speed (i.e., a lower gravitational force), and have been widely utilized to study the long-term stability of suspensions. Recently, AC has been extended for quantitative studies of colloidal nanoparticle sedimentation and particle-particle interactions. ${ }^{36-38} \mathrm{AC}$ has the advantage of enabling explorations of nanoparticle colloidal stability on a short timescale compared to batch sedimentation testing (matter of hours 
versus months).

$\mathrm{AC}$ and the available theoretical sedimentation models are mainly used to study the particle sedimentation behavior of well-defined systems, i.e., particles with a narrow size distribution or known bimodal particle systems. ${ }^{36-38}$ Here, we explore the utilization of AC to study the stability of silicon nanoparticle dispersion with a much more complex shape and surface chemistry to evaluate its potential use and to speed up specific particle system stability tests. The motivation of this study, as previously mentioned, has been the development of silicon nanoparticle-based inks suitable for inkjet printing. Stability is defined here as a desirable shelf life exceeding two months. We investigate the sedimentation behavior of SiNPs in various alcohols and glycols by using Stokes' law and performing $\mathrm{AC}$ measurements. In addition, we report the results of batch sedimentation studies.

\subsection{Results and Discussion}

\subsubsection{SiNP Characterization}

We determined the particle geometry and surface chemistry by transmission electron microscope (TEM), dynamic light scattering (DLS), X-ray photoelectron spectroscopy (XPS), and Fourier-transform infrared (FTIR) spectroscopy prior to performing the sedimentation studies. Figure 3.3a shows an TEM image of SiNPs deposited on a carbon/copper grid. The magnified TEM image (Figure 3.3b) shows that the primary particles contain amorphous and crystalline domains. The crystalline domains have various orientations, with prominent [111] $(0.313 \mathrm{~nm})$ and [220] $(0.210 \mathrm{~nm})$ planes, as observed in the TEM image. ${ }^{39}$ This results from the collisions of nucleating materials in the gas phase fusing to form primary particles during synthesis. ${ }^{40}$

The TEM images reveal that at the edge of the SiNPs, there is a visible, thin amorphous shell with a thickness of approximately $5 \AA$. From complementary experiments (XPS and IR spectroscopy), we conclude that this layer consists of oxidized silicon, i.e., $\mathrm{SiO}_{2}$. Due to the high reactivity of SiNPs surface to oxygen, the pristine $\mathrm{SiNP}$ surface was instantly oxidized upon exposure to air, leading to the observed $\mathrm{Si} / \mathrm{SiO}_{2}$ coreshell structure. This thin $\mathrm{SiO}_{2}$ shell protects the SiNPs from further oxidation and maintains their chemical stability under ambient conditions. ${ }^{41}$ Furthermore, from Figure $3.3 \mathrm{a}$, it is clear that the primary particles are aggregated through necking (sintering bridges) to form secondary particles consisting of several tens of primary particles. The primary SiNPs have a sphericity close to unity and an average diameter of $50 \pm 17 \mathrm{~nm}$ 
(see Figure 3.3c). Due to the aggregated structure of the SiNPs, characterization of the secondary particle size and shape by microscopy imaging was challenging (see Figure 3.3a and $\mathrm{d})$.
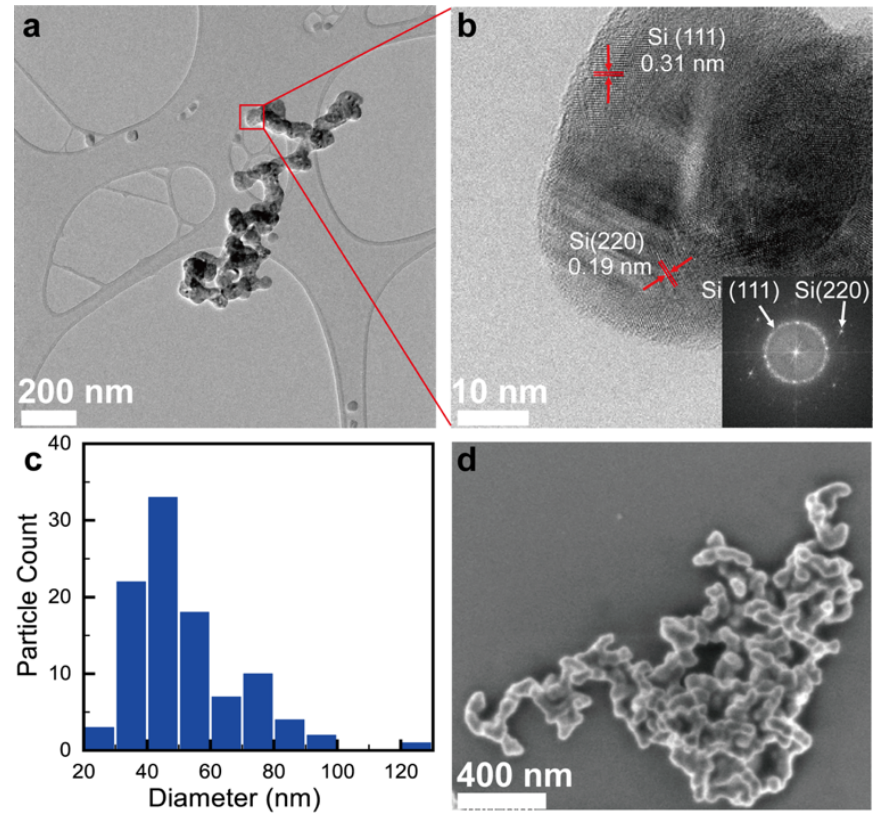

Figure 3.3 a) TEM image of SiNPs deposited on a carbon/copper grid, b) magnified image of the area in the red box shown in (a), the inset shows a fast Fourier transform pattern, c) particle size distribution of the primary SiNPs $(n=100)$ and d) SEM image of SiNPs placed on a graphite holder.

Thus, alternative approaches for size determination, such as DLS, have been employed. Values of the hydrodynamic diameter of the SiNPs were obtained for aqueous dispersions. The diameters determined by TEM and DLS should not be the same value by definition, and we consider DLS data to be a good measure of the diameter of the agglomerated particles.

For the DLS measurements, SiNPs were dispersed in Milli-Q water at a concentration of $25 \mathrm{ppm}$ via intense sonication. The hydrodynamic diameter of the diluted SiNPs in water (see Figure 3.4) shows a distribution ranging from 110 to $550 \mathrm{~nm}$, with an average hydrodynamic diameter of $287 \mathrm{~nm}$ (PDI 0.235). We note that for the other solvents used the hydrodynamic radius distribution of the SiNPs was in the same range. For reasons of simplicity we therefore used the values obtained for the water based dispersions in the following calculations. 


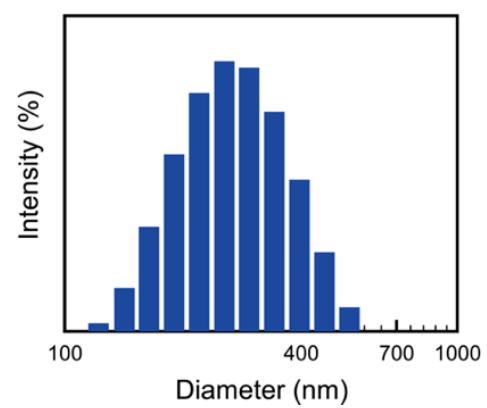

Figure 3.4 Hydrodynamic diameter obtained by DLS measurements of SiNPs dispersed in water at a concentration of $25 \mathrm{ppm}$.

Stokes' law was applied to calculate the sedimentation velocity $(v)$ of SiNP in suspensions. The values of $v$ of SiNPs in methanol, ethanol, 1-hexanol, isobutanol, ethylene glycol, and diethylene glycol under Earth's gravity $\left(v_{\mathrm{g}}\right)$ and accelerated centrifugal field $(1300 * \mathrm{~g})\left(v_{a c}\right)$ are presented in Table 3.1. Moreover, the times $(t)$ for SiNPs to settle $1 \mathrm{~cm}$ are shown, as well.

Table 3.1 The sedimentation velocity of SiNPs under the Earth's gravity (ve) and in AC (vac); and the corresponding time for SiNPs to settle $1 \mathrm{~cm}$ te and tac. The values of the average hydrodynamic diameter of SiNPs from the DLS measurements were used for the calculations. The parameters used to perform the calculations can be found in Table S3.1.

\begin{tabular}{ccccccc}
\hline & methanol & ethanol & 1-hexanol & isobutanol & $\begin{array}{c}\text { ethylene } \\
\text { glycol }\end{array}$ & $\begin{array}{c}\text { diethylene } \\
\text { glycol }\end{array}$ \\
$\boldsymbol{v}_{\boldsymbol{e}}\left(\mathbf{n m ~ s}^{-1}\right)$ & 40.6 & 27.6 & 1176 & 13.4 & 3.3 & 2.4 \\
$\boldsymbol{t}_{\boldsymbol{e}}(\boldsymbol{h})$ & 69 & 100.5 & 237.0 & 206.8 & 853.5 & 1147.4 \\
$\boldsymbol{v}_{\boldsymbol{a c}}\left(\boldsymbol{\mu m} \boldsymbol{~ s}^{-1}\right)$ & 52.7 & 35.9 & 15.2 & 17.5 & 4.2 & 3.1 \\
$\boldsymbol{t}_{\boldsymbol{a c}}(\min )$ & 3.2 & 4.7 & 10.9 & 9.5 & 39.4 & 53.0
\end{tabular}

As previously mentioned, corresponding colloid stability theories often assume narrow (or even monomodal) size distributions. Due to the substantial width of the particle distribution functions obtained here, we speculate that the theory predictions such as Stokes' law could deviate from the actual sedimentation behavior. In sections 3.2 and 3.3 we will compare the results from Stokes' law predictions with the AC and the batch sedimentation tests. 

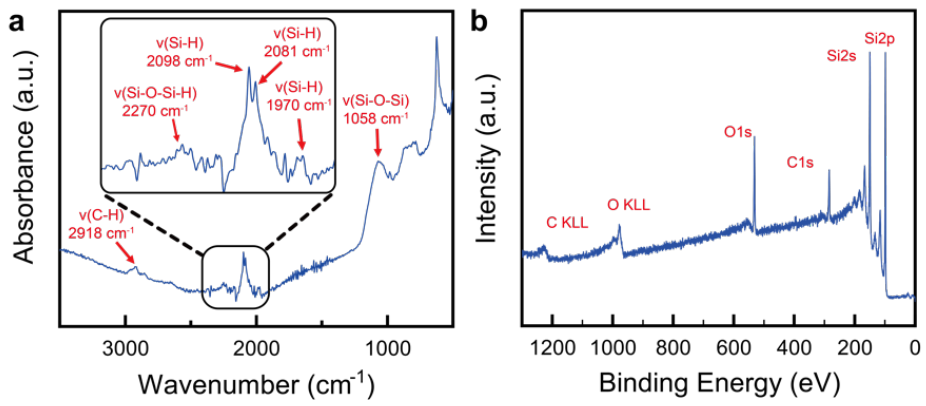

Figure 3.5 a) Single reflection ATR-FTIR spectrum and b) XPS overview spectrum of SiNPs.

In addition to morphology, surface chemistry is also an essential factor that influences colloidal stability. We determined the surface composition of SiNPs' by FTIR spectroscopy and XPS measurements. Figure 3.5a shows an infrared spectrum of the SiNPs. The sharp peak observed at $1058 \mathrm{~cm}^{-1}$ in the FTIR spectrum corresponds to Si-OSi stretching absorption, which agrees with the presence of the $\mathrm{SiO}_{2}$ layer observed through the TEM analysis (see Figure 3.3a). The most interesting absorbance bands in Figure 3.5a are visible at $1970 \mathrm{~cm}^{-1}, 2081 \mathrm{~cm}^{-1}, 2098 \mathrm{~cm}^{-1}$, and $2270 \mathrm{~cm}^{-1}$, corresponding to the $\mathrm{Si}-\mathrm{H}$ stretching vibrations of $\mathrm{H}-\mathrm{Si}\left(\mathrm{Si}_{3}\right), \mathrm{H}-\mathrm{Si}\left(\mathrm{Si}_{2} \mathrm{O}\right), \mathrm{H}-\mathrm{Si}\left(\mathrm{SiO}_{2}\right)$, and $\mathrm{H}-\mathrm{Si}\left(\mathrm{SiO}_{3}\right)$ bonds, respectively. ${ }^{42}$ The presence of $\mathrm{H}-\mathrm{Si}-\mathrm{O}$ bonds indicates that not all the $\mathrm{Si}-\mathrm{H}$ bonds were oxidized. It is worth noting that the small peak observed at $2918 \mathrm{~cm}^{-1}$, ascribed to $\mathrm{v}(\mathrm{C}-\mathrm{H})$ absorbance, indicates that organic contaminants are present at the particle surface.
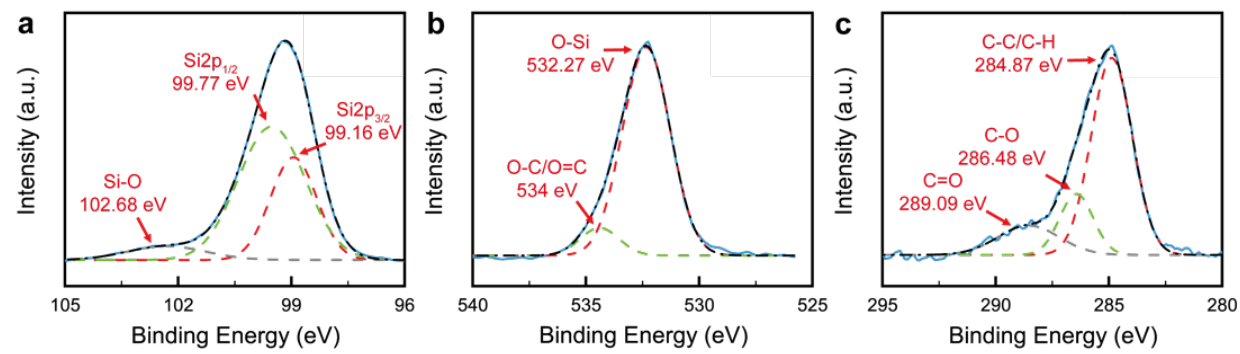

Figure 3.6 a) Si2p, b) O1s and c) C1s XPS core-level spectra of SiNPs. The dotted lines show the deconvolution fitting of the core-level spectra to identify the bonds present.

The XPS spectrum (Figure 3.5b) confirms that SiNPs contain $\mathrm{Si}, \mathrm{O}$, and C. The boron dopant $(\sim 190 \mathrm{eV})$ is not visible in the XPS spectrum. The missing B peak is ascribed to the relatively low doping level $\left(10^{18}\right.$ atom $\left.\mathrm{cm}^{-3}\right)$ and the tendency of $\mathrm{B}$ atoms to migrate to the SiNPs' cores. ${ }^{43}$ In addition to the low B surface concentration, the overlap of the solid Si2s band with the B1s band makes it difficult to detect boron with XPS. According to the XPS analysis, the SiNPs consist of $\sim 26.4 \%$ carbon atoms, $\sim 18.6 \%$ oxygen atoms, 
and $55.0 \%$ silicon atoms. We noted that the carbon content was too high to be ascribed to the organic contaminants typically observed solely in XPS measurements. Therefore, the $\mathrm{C}, \mathrm{O}$, and $\mathrm{Si}$ core-level spectra were examined in detail to determine the nature of the carbon content.

Figure 3.6 shows the Si 2p, O 1s, and C 1s core-level spectra of the SiNPs. In the Si $2 p$ core-level spectrum (Figure 3.6a), the dominant bands observed are $99.16 \mathrm{eV}$ and $99.77 \mathrm{eV}$, corresponding to the characteristic binding energies for elemental silicon. ${ }^{44}$ The band at $102.68 \mathrm{eV}$, which corresponds to $\mathrm{Si}-\mathrm{O}$ bonds, is expected due to the presence of the oxidized silicon layer. ${ }^{44}$ This is confirmed by the peak at $532.27 \mathrm{eV}$ in the $\mathrm{O} 1 \mathrm{~s}$ core-level spectrum (Figure 3.6b). ${ }^{45}$ The observed difference between the Si-Si and Si-O peak areas in Figure 3.6a indicates that only a small amount of Si-Si bonds was oxidized. This low degree of oxidation is in agreement with the observed thickness of the oxide layer ( $5 \AA$ ) in the TEM images (see Figure 3.3). In the $\mathrm{C} 1$ s core spectrum (Figure 3.6c), most of the $\mathrm{C}$ atoms present were in the form of C-C/C-H (binding energy $284.87 \mathrm{eV}$ ), which is typical for organic contamination on the particle surface. ${ }^{46}$ In addition, some $\mathrm{C}$ atoms were bonded to $\mathrm{O}(\mathrm{C}-\mathrm{O} / \mathrm{C}=\mathrm{O}$ binding energies of $286.48 \mathrm{eV}, 289.09 \mathrm{eV}$, and 534 $\mathrm{eV}$, see Figure 3.6b and Figure 3.6c). ${ }^{46}$ The missing Si-C peak in both the Si and C 1s core-level spectra $(100.4 \mathrm{eV}$ and $283.1 \mathrm{eV})$ indicates that there is no tracible $\mathrm{C}-\mathrm{Si}$ bonding. Based on these results, we conclude that the $26.4 \%$ carbon content measured in XPS originates from the presence of organic contamination that is presumably partially present at the particle surface.

Thus, in addition to the irregular shape and broad size distribution, the complex surface chemistry of the SiNPs could make any theoretical prediction of their sedimentation behavior in different solvents deviate from the actual situation. Therefore, we employed AC and batch sedimentation measurements to assess the colloidal stability of the SiNPs in a selection of various alcohols and glycols. The results are presented and discussed in the next section.

\subsubsection{AC to Study SiNP Sedimentation Behavior in Various Solvents}

In this section, we discuss the results of the sedimentation experiments under centrifugal force and in the gravitational field. First, we discuss data obtained by an analytical centrifuge. Figure 3.7 shows the temporal evolution of the light transmittance of $0.5 \mathrm{wt} \%$ SiNP suspensions in methanol, 1-hexanol, ethylene glycol, ethanol, isobutanol, and diethylene glycol obtained by AC measurements. To quantify the particle sedimentation from the analytical centrifuge results, the instability indexes of SiNPs in different solvents were extrapolated from the light transmittance profiles of the 
suspensions between $\mathrm{r}=115 \mathrm{~mm}$ and $\mathrm{r}=125 \mathrm{~mm}$ (see Figure 3.8). One can readily observe that the SiNP dispersion in ethylene glycol and diethylene glycol has the best colloidal stability among the tested solvents under the centrifugal force field used. The instability index for ethylene glycol and diethylene glycol suspensions is well below 0.02 throughout the AC experiment. This means that even after $10 \mathrm{~h}$ of centrifugation, most of the particles remained well dispersed in ethylene glycol and diethylene glycol.
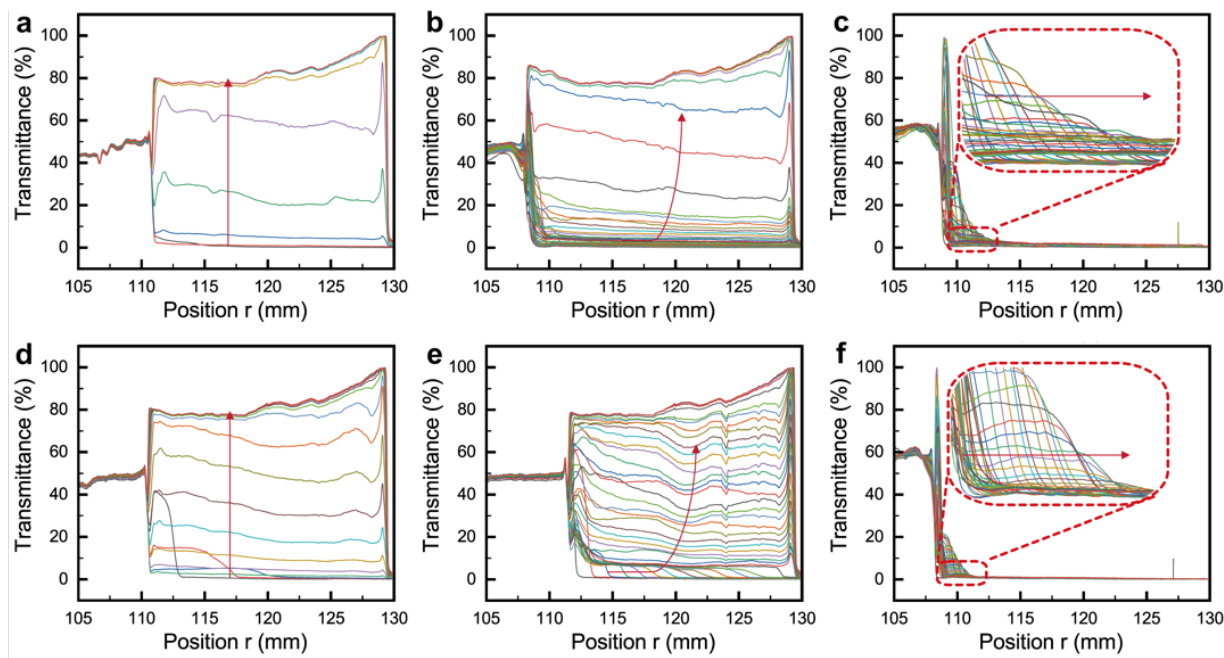

Figure 3.7 Time dependent AC light transmittance profile measurements of SiNPs dispersed in methanol (a), 1-hexanol (b), ethylene glycol (c), ethanol (d), isobutanol (e), and diethylene glycol. For each dispersion, the SiNP concentration was $0.5 \mathrm{wt} \%$. The $r$ indicates the distance to the rotation center. To assist the reader, the red arrow in each graph indicates the evolution of time.

Without the presence of electrolytes, van der Waals (vdW) attraction is one of the major causes of particle aggregation. ${ }^{47}$ The vdW attraction between two identical microbodies in a dense medium can be quantified via the Lifshitz theory of vdW forces. ${ }^{48}$ According to the Lifshitz theory and the Hamaker equation, the vdW force intensity strongly depends on the polarizability of the embedding medium. ${ }^{49}$ From the ClausiusMossotti equation, it is known that the polarizability $\alpha \propto(\varepsilon-1) /(\varepsilon+2)(\varepsilon$ is the relative permittivity/dielectric constant of the medium). ${ }^{50}$ Therefore, if we only consider vdW forces, the attractive forces between particles in ethylene glycol $(\varepsilon=37.6$ at $303 \mathrm{~K})$ and diethylene glycol $(\varepsilon=29.1$ at $303 \mathrm{~K})$ are weaker than those of 1-hexanol and isobutanol ( $\varepsilon=12.7$ and $\varepsilon=17.4$ at $303 \mathrm{~K}$, respectively). This theory would predict that the particles in ethylene glycol and diethylene glycol are less susceptible to aggregation. Additionally, the relatively high viscosities of ethylene glycol and diethylene glycol $(16.3 \mathrm{mPa} \cdot \mathrm{s}$ and $21.8 \mathrm{mPa} \cdot \mathrm{s}$ at room temperature, respectively) reduced Brownian motion, i.e., reduce the 80 
collision rate between particles. Thus, weak vdW attraction and a low collision rate between SiNPs reduce aggregation in these solvents. ${ }^{49}$ The high viscosity also results in a large friction drag force that acts on the settling particles against the settling direction. A considerable friction drag effectively reduces the sedimentation velocity for the particles in the suspension. ${ }^{24}$ Therefore, when suspended in ethylene glycol and diethylene glycol, the agglomeration rate and sedimentation velocity of the SiNPs should be significantly reduced in the centrifugal field (AC). These predictions corroborate the observations satisfactorily. Only a tiny fraction of the particles/agglomerates with large radii sedimented, and the small particles remained dispersed for a relatively long time.

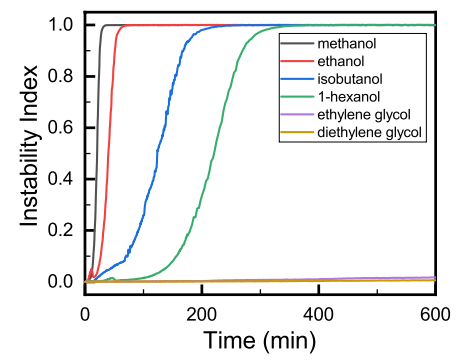

Figure 3.8 Instability index values of SiNPs dispersed in methanol, ethanol, 1-hexanol, isobutanol, ethylene glycol, and diethylene glycol between $r=115 \mathrm{~mm}$ and $r=125 \mathrm{~mm}$ plotted against time.

Methanol and ethanol ( $\varepsilon=31.7$ and $\varepsilon=23.6$ at $303 \mathrm{~K}$, respectively) have dielectric constants comparable to those of glycols, indicating relatively weak vdW attraction between particles compared to 1-hexanol and isobutanol. We observed a fast and nearly uniform concentration decrease along the length of the test tubes for methanol and ethanol suspensions during the $\mathrm{AC}$ measurements. The light transmittance for methanol and ethanol increased to more than $80 \%$ within one hour and remained constant for the rest of the AC measurements. Therefore, most of the particles in methanol and ethanol sedimented within one hour after the centrifugal force field was applied and these dispersions were considered to be unstable. Similar to those of glycols, the high dielectric constants of methanol and ethanol result in insufficient repulsive forces to successfully stabilize the respective SiNP dispersions. The relatively low viscosities of methanol and ethanol $(0.6 \mathrm{mPa} \cdot \mathrm{s}$ and $1.2 \mathrm{mPa} \cdot \mathrm{s}$ at room temperature, respectively) are ineffective in reducing Brownian motion. As a result, the SiNPs in methanol and ethanol have more frequent collisions, eventually leading to particle aggregation. Additionally, the relatively low viscosities of ethanol and methanol result in a smaller friction drag force. The low friction drag results in an increased sedimentation velocity for the particles dispersed in 
methanol/ethanol compared to those dispersed in ethylene glycol and diethylene glycol. From the transmittance and instability index profiles of the SiNP methanol and ethanol dispersions, it is obvious that the particle concentrations (light transmittance) decreased much more slowly at the early stages of the sedimentation process and decreased at a faster rate as time proceeded. This observation is explained by realizing that smaller particles and solvent molecules behave similarly to an effective viscous fluid. ${ }^{51}$ This fluid provides a higher friction drag force that slows down sedimentation and generates a backflow when large particles/aggregates move towards the bottom. ${ }^{52}$ As a result of the backflow, smaller particles flow upwards together with the solvent molecules, resulting in a uniform decrease in particle concentration over the sample length instead of hardsphere sedimentation. ${ }^{52}$ For ethylene glycol and diethylene glycol-based SiNP suspensions, we did not observe a uniform decrease in particle concentration due to the high effective viscosity, which suppressed the backflow. ${ }^{52}$

For 1-hexanol and isobutanol SiNP suspensions, the temporal evolution of light transmittance profiles suggests that the particle sedimentation process in these suspensions can be interpreted by a combination of the effects that govern the settling behavior in ethylene glycol/diethylene glycol and methanol/ethanol. At early stages of particle sedimentation, the effective viscosity of the suspensions is high enough to suppress/reduce backflow, resulting in a sedimentation profile similar to that of glycols. ${ }^{51,53}$ As the particle concentration decreases and large particles settled to the bottom during the AC measurement, the suspension's effective viscosity decreases, resulting in an increased backflow that reduces particle sedimentation. Therefore, for 1hexanol and isobutanol SiNP dispersions, the sedimentation shifts from glycol-like behavior during the early stages of the experiment to methanol-like behavior, and uniform particle concentration decreases during the later stages of the measurement.

The AC results shown here suggest that solvents such as ethylene glycol and diethylene glycol provide the best colloidal stability for the SiNPs, which is in good agreement with Stokes' Law predictions (see Table 3.1). Therefore, AC is a useful tool for analyzing the colloidal stability of particle suspensions related to viscosities and densities. However, to determine the shelf life of particle suspensions, other factors like aggregation, chemical stability are also crucial and should not be ignored. In the next section, we will compare the AC stability tests with batch sedimentation tests.

\subsubsection{Batch Sedimentation Evaluation of SiNP Dispersions}

Figure 3.9 shows a series of images of the batch sedimentation measurements of SiNPs dispersed in methanol, ethanol, 1-hexanol, isobutanol, ethylene glycol, and 82 
diethylene glycol recorded over 90 days. During these experiments, only gravitational forces were present. For the SiNP dispersions in isobutanol and 1-hexanol, the layer near the liquid-gas interface became brighter at days 5 and 10, respectively. Following these initial changes, the SiNP dispersions in isobutanol and 1-hexanol remained unchanged until day 40. After 40 days, clear sedimentation was observed and progressed over time. We note that the camera could not capture particle sedimentation at the bottom of the vials for isobutanol and 1-hexanol and that these results reflect observations made during our regular visual inspections. For the isobutanol and 1-hexanol SiNP dispersions, we explain these results by observing the initial sedimentation of larger particles ( $<10$ days), after which the smaller NPs were relatively stable for a few weeks ( $<40$ days) until they eventually agglomerated and the dispersion became unstable ( $>40$ days).

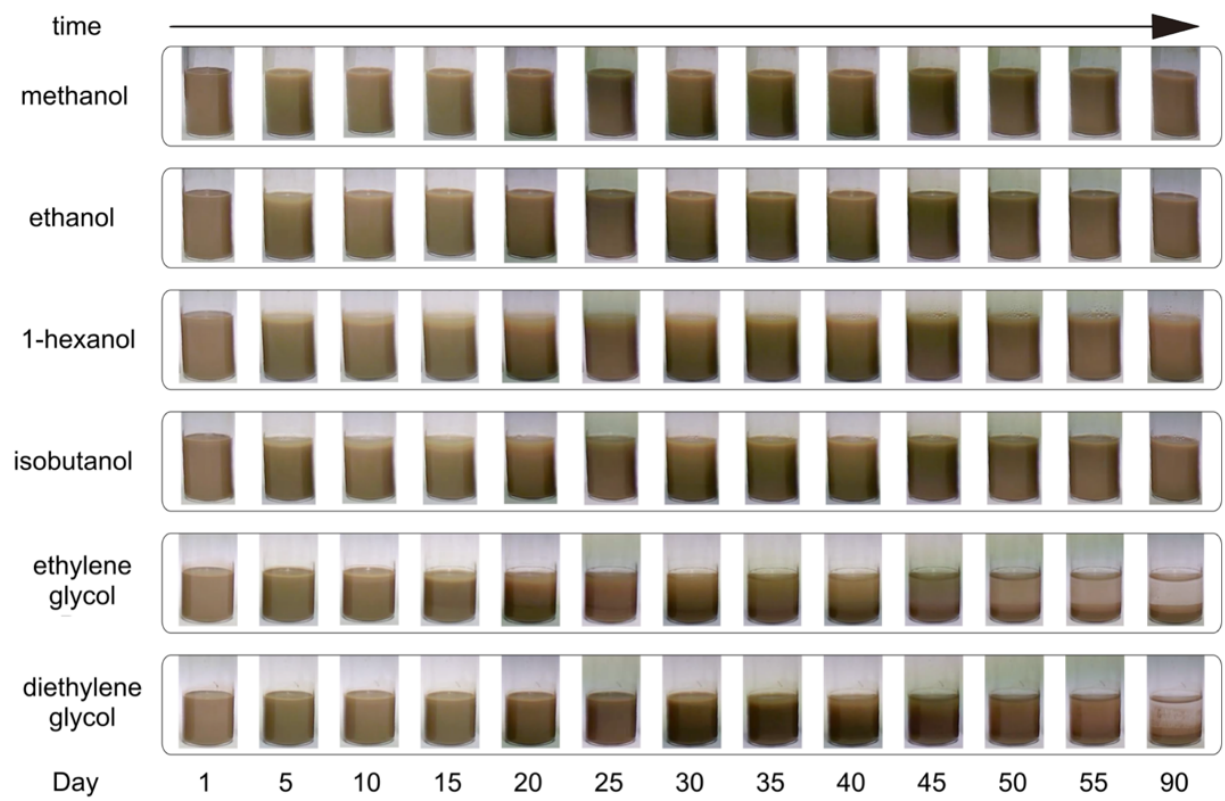

Figure 3.9 Images of batch sedimentation measurements of SiNP suspensions in methanol, ethanol, 1-hexanol, isobutanol, ethylene glycol, and diethylene glycol recorded with a surveillance camera for 90 days.

For the SiNP dispersions in methanol and ethanol, we noticed no significant sedimentation of particles or changes in the dispersions' appearance during the observation interval. Due to the relatively low viscosity of methanol and ethanol (compared to the other solvents used), the larger particles in methanol and ethanol sedimented much faster, transitioning from the more homogenous, stable dispersion at the start of the experiment. ${ }^{51}$ In addition, it is known that the drag force is proportional to 
the solvent viscosity, ${ }^{24}$ i.e., the particles that remain in the ethanol and methanol suspensions should have smaller hydrodynamic diameters than those in isobutanol, 1hexanol, ethylene glycol, and diethylene glycol after the larger particles settled. These smaller particles need more time to agglomerate and reach a critical size before the dispersion becomes unstable. ${ }^{24}$ According to Stokes' law predictions (see Table 3.1), even the smallest particles in methanol and ethanol should have settled at least $1 \mathrm{~cm}$ after 16 and 23 days, respectively. However, Stokes' law is only applicable for spherical particles, and as shown in Figure 3.3, the SiNPs used in this study are clearly not spherical. Due to the aggregated morphology of the SiNPs, the real gravitational force and drag force can deviate from the theoretical values. Moreover, the alcohol molecules like methanol and ethanol can bond (hydrogen bonding) with their constituent oxygen atoms to the particle surface to form a solvation shell. ${ }^{54}$ This solvation shell could raise the solvation force between particles and effectively reduce the agglomeration rate. This explains why the dispersions of SiNPs in methanol and ethanol were stable for the entire duration of the batch sedimentation experiments.

The results obtained from the batch sedimentation experiments seem to also differ from the results of the AC measurements of the methanol and ethanol SiNP dispersions. When the suspensions are in a centrifugal field, the gravitational force and friction drag increase, resulting in increased sedimentation velocity. However, the excess pressure applied to the suspension from AC could promote particle aggregation, especially for low-density solvents like methanol and ethanol. ${ }^{54}$ Moreover, we speculate that the Brownian motion, which solely depends on particle size and fluid properties, was not influenced by the centrifugal field. ${ }^{24}$ Thus, in AC measurements, particle collision and agglomeration resulting from Brownian motion are not enhanced. As such, the influence of Brownian motion on colloidal stability is not captured by the $\mathrm{AC}$ measurements in the RPM range we used.

The ethylene glycol and diethylene glycol SiNP suspensions were stable during the first 15 and 30 days of the batch sedimentation experiments, respectively. For ethylene glycol, the first trace of sedimentation was observed on day 15 , followed by relatively rapid clearance of SiNPs from the dispersion in the following days. On day 40, which is only 25 days after the first trace of sedimentation, most of the SiNPs in ethylene glycol settled at the bottom of the test vial as a thick layer of SiNPs. The supernatant of the suspension was almost transparent. We observed similar behavior for SiNPs dispersed in diethylene glycol, for which the first signs of particle sedimentation were observed at day 30 , and most of the particles were sedimented at day 50 . 
A striking difference that was observed in the SiNP dispersion stability in ethylene glycol and diethylene glycol during $\mathrm{AC}$ and batch sedimentation experiments was not expected based on the initial observations of the $\mathrm{AC}$ measurements and the accompanying explanation for the high dispersion stability. ${ }^{23,24}$ In addition, unlike for methanol and ethanol suspensions, Stokes' law overestimated the particle stability in ethylene glycol and diethylene glycol. According to Stokes' law predictions, the smallest particles in the ethylene glycol and diethylene glycol will take 197 and 265 days to settle over $1 \mathrm{~cm}$ under the Earth's gravity. Two hydroxyl groups in the glycol molecules may bond to different particles (e.g., via hydrogen bonding) and act as a bridging agent. The bridging effect would accelerate particle aggregation and sedimentation. In the next section, the colloidal dispersion stability is investigated in more depth to explain the observed poor stability of the SiNP dispersion in ethylene glycol and diethylene glycol.

\subsubsection{Ethylene Glycol and Diethylene Glycol Stability in the Presence of SiNPs}

The supernatants from ethylene glycol and diethylene glycol SiNP suspensions were diluted 10 times with Milli-Q water and measured with a $\mathrm{pH}$ meter at days 30 and 90. The $\mathrm{pH}$ decreased from 5.9 to 5.2 and 6.1 to 5.5 for ethylene glycol and diethylene glycol supernatants, respectively. The changes in $\mathrm{pH}$ over time indicated a possible change in the supernatant composition, i.e., the solvent composition, which could explain the unexpected sedimentation behavior for the SiNPs in ethylene glycol and diethylene glycol. To further elucidate the reason behind the abnormally fast sedimentation of the ethylene glycol and diethylene glycol suspensions during the batch sedimentation experiments, the supernatants of ethylene glycol and diethylene glycol suspensions were collected and characterized by FTIR and ion chromatography (IC).

Figure 3.10a shows FTIR spectra of the ethylene glycol and diethylene glycol supernatants collected on day 90. For comparison, FTIR spectra of the pure solvents stored in glass vials for 90 days are also shown. From the FTIR spectra, it is readily observed that for both ethylene glycol and diethylene glycol, the spectra of the supernatants and the corresponding pure solvents are almost identical except for the presence of two small peaks at $1650 \mathrm{~cm}^{-1}$ and $1720 \mathrm{~cm}^{-1}$, which correspond to $\mathrm{C}=\mathrm{C}$ and $\mathrm{C}=\mathrm{O}$ stretching absorbances, respectively. ${ }^{55}$ The presence of the $\mathrm{C}=\mathrm{C}$ and $\mathrm{C}=\mathrm{O}$ groups could be ascribed to the oxidation products of ethylene glycol and diethylene glycol, such as formic acid, glycolic acid, and acetaldehyde. ${ }^{56}$ 

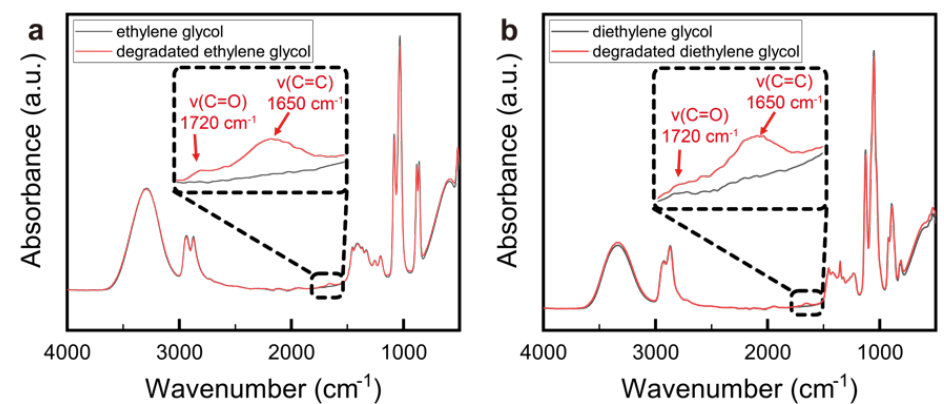

Figure 3.10 Single reflection ATR-FTIR spectra of a) ethylene glycol and b) diethylene glycol supernatants collected on day 90 of the SiNP dispersion batch sedimentation experiment. FTIR spectra for the pure solvents stored in a glass bottle for 90 days are also shown.

To further clarify the potential presence of oxidation products in the supernatant, ion chromatography was applied. According to literature, the main oxidation products of ethylene glycol are oxalic acid, glycolic acid, and formic acid. ${ }^{56}$ Therefore, the ion chromatograph was calibrated to look for the presence of the anions from the corresponding degradation products. The resulting chromatographs are shown in Figure 3.11 .
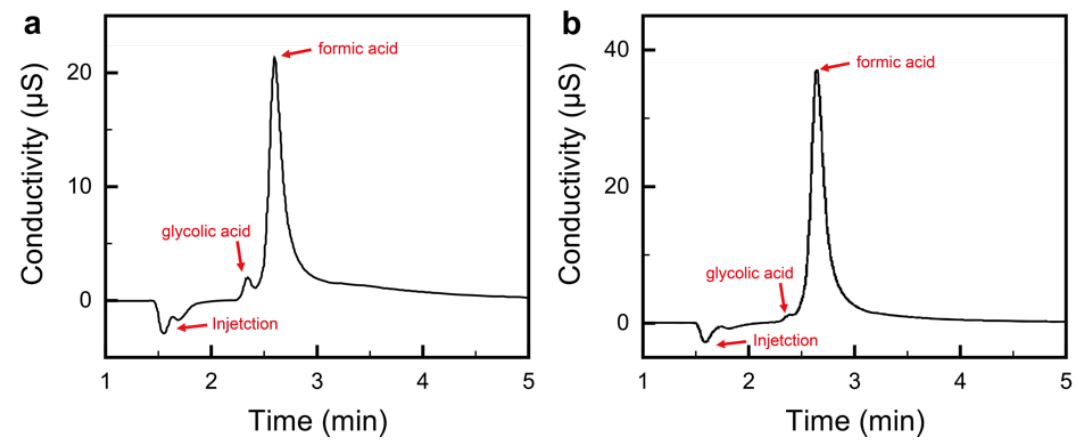

Figure 3.11 Ion chromatography anion analysis for the supernatants of a) ethylene glycol and b) diethylene glycol collected at day 90 of the SiNPs dispersion batch sedimentation experiments.

In Figure 3.11, the presence of glycolic acid and formic acid in the supernatants of ethylene glycol and diethylene glycol is clearly visible. The chemical structure of formic acid and glycolic acid also explains the $\mathrm{C}=\mathrm{O}$ and $\mathrm{C}=\mathrm{C}$ absorbances observed with FTIR spectroscopy (see Figure 3.10). We note that no evidence of oxidation was observed with FTIR spectroscopy for the pure solvent stored in its original vial for the duration of the batch sedimentation experiments. These results confirm that ethylene glycol and diethylene glycol were partially oxidized/degraded in the presence of SiNPs during the 
batch sedimentation experiment on a relatively short time scale. Consequently, the acidic degradation products that release free ions can be absorbed onto the SiNP surface and form an electric diffusion layer around the SiNPs. Through the degradation process, the increase in ion concentration decreases the electric double layer thickness, resulting in fast aggregation and sedimentation of the SiNPs. ${ }^{57}$ In a simple control experiment, we slightly acidified freshly made SiNP dispersions in ethylene glycol and diethylene glycol dispersions, resulting in rapid destabilization of the respective dispersions. The reason why the SiNPs induced partial degradation of ethylene glycol and diethylene glycol remains unclear.

Overall, in combination with the previously mentioned limitations of AC to study complex particle dispersion stability, we conclude that although $\mathrm{AC}$ is a valuable and proven technique for reducing the time required for colloidal stability studies in research and product development, ${ }^{36-38}$ it has limitations. In particular, shortcomings must be considered when evidence of particle/solvent system chemical instability is sought for, that occurs on time scales that substantially exceed those of the AC measurements. Without prior knowledge of such processes, the more time-consuming batch sedimentation experiments remain of pivotal importance.

\subsection{Conclusion}

The colloidal stability of nonspherical SiNPs in various solvents was investigated by $\mathrm{AC}$ and batch sedimentation measurements. Due to the complex geometry and surface chemistry of SiNPs, differences in dispersion stability from Stokes' law predictions, AC and batch sedimentation measurements were observed for SiNP dispersions in various alcohols and glycols. These differences result from the applicability of Stokes' law and AC measurements on particles with complex geometry and surface chemistry. Both Stokes' law and AC focus on/magnify the influence of viscosity and density on the colloidal stability of particle suspensions. Other factors like i) the influence of Brownian motion on particle agglomeration and sedimentation, ii) the effect of backflow on local particle concentration, and iii) the possible long-term chemical interactions between particles and solvents are underestimated when using Stokes' law predictions or AC measurements. These results underline the importance of conducting time-consuming batch sedimentation experiments to determine the shelf life of colloidal dispersions. Furthermore, future efforts to speed up dispersion stability tests for more complex nanoparticle dispersions must be directed toward identifying accelerated test conditions where the impact of these effects are neglectable. 


\subsection{Materials and Methods}

Materials. Boron-doped silicon nanoparticles with a boron doping level of $5 * 10^{18}$ atoms $\mathrm{cm}^{-3}$ were a gift from University Duisburg-Essen, Germany. The particles were kept under ambient conditions and used as received. The nanopowder was synthesized via a gas-phase route in a hot-wall reactor, allowing the large-scale production of nanomaterials. A detailed description of the synthesis was reported by Hülser et al. ${ }^{58}$ From now on, we will refer to these nanoparticles as SiNPs. Analytical-grade absolute methanol, absolute ethanol, isobutanol, and acetonitrile were purchased from Merck (Darmstadt, Germany). 1-Hexanol (anhydrous, 99\%), ethylene glycol (anhydrous, 99.8\%), and diethylene glycol (ReagentPlus, 99\%) were purchased from Sigma Aldrich (St. Louis, MO, USA). Milli-Q water was prepared by a Millipore Synergy System (Billerica, MA, USA). Unless otherwise mentioned, all chemicals were used as received.

Preparation of Colloidal Suspensions. Methanol, ethanol, isobutanol, 1-hexanol, ethylene glycol, and diethylene glycol were selected as solvents to prepare the SiNP dispersions to cover a broad range of dielectric constants and viscosities. The dispersions were prepared by dispersing $0.05 \mathrm{~g}$ of SiNPs in $9.95 \mathrm{~g}$ of each solvent mentioned above, followed by $3 \mathrm{~h}$ of sonication (BRANSON 2510, Canada) to form uniform suspensions. The dynamic viscosities of the prepared particle suspensions and viscosity of the pure solvents were measured by a digital viscometer (LVTDV-I, Brookfield Engineering, AMETEK, Berwyn, Pennsylvania, U.S.). Prior to the measurements, the solvents and suspensions were sealed in the bottle and place in the fume hood for an hour to reach thermal equilibrium.

Characterization of Silicon Nanoparticles. Transmission electron microscopy (TEM) was performed by an FEI Tecnai 20 system (FEI, Eindhoven, the Netherlands). A few drops of diluted particles dispersed in ethanol were deposited on the carbon side of a carbon/copper grid (HC200-Cu) (EMS, Germany) before imaging. Scanning electron microscope (SEM) was performed by a JEOL Field Emission JSM-633OF system (JEOL Benelux, Nieuw-Vennep, the Netherlands). Fourier transform infrared (FTIR) spectra were collected with a Bruker ALPHA single attenuated total reflection (ATR) FTIR spectrometer equipped with an ATR single reflection diamond crystal (Bruker Optic $\mathrm{GmbH}$, Ettlingen, Germany). FTIR spectra were collected in the range of $400-4000 \mathrm{~cm}^{-1}$ (spectral resolution of $4 \mathrm{~cm}^{-1}, 512$ scans). The background spectra were recorded against air. The hydrodynamic radius of the SiNPs in Milli-Q water was determined using dynamic light scattering (DLS) with a Malvern Zetasizer Nano-ZS (Malvern Instruments, Malvern, United Kingdom). A PHI Quantera II (Physical Electronics, Minnesota, US) 
equipped with a monochromatic $\mathrm{Al} \mathrm{K} \alpha \mathrm{X}$-ray source $(1486.6 \mathrm{eV})$ was used to perform $\mathrm{X}$-ray photoelectron spectroscopy (XPS). The angle for the X-ray beam and detector was set at $45^{\circ}$.

Stokes' Law Prediction. Stokes' law for spherical objects was applied to estimate the sedimentation velocity of SiNPs in as-prepared suspensions:

$$
v=\frac{2 r^{2}\left(\rho_{2}-\rho_{1}\right)}{9 \eta}
$$

Where $\mathrm{r}$ is the average hydrodynamic radius of SiNPs (measured with DLS), $\rho_{1}$ and $\rho_{2}$ are the density of solvent and SiNPs, respectively. $\eta$ is the dynamic viscosity of the suspensions.

Analytical Centrifugation. The centrifugal sedimentation experiments were performed using a low-velocity AC (LUMiFuge, LUM GmbH, Germany). The particle concentrations of the suspensions were monitored qualitatively by obtaining the transmitted light intensity against time and position over the sample length (see Figure 3.2). The particle suspensions were transferred into a disposable polycarbonate cell followed by insertion into the LUMiFuge. The LUMiFuge was set at $3000 \mathrm{rpm}(\sim 1300$ $\mathrm{g}$ ), and the data collection time was $12 \mathrm{~h}$. The transmission profiles were collected every 5 minutes.

Instability Index. The value of the instability index can be calculated from the AC results. ${ }^{59}$ The light transmittance of the sample at $\mathrm{t}=0$ is defined as $\mathrm{T}_{1}(\mathrm{r}) . \mathrm{r}$ is the distance between a given position and the rotation center. The value of clarification (change of particle concentration) $T_{i}^{\text {diff }}$ is defined as $\mathrm{T}_{\mathrm{i}-} \mathrm{T}_{1}$. The total clarification $\Delta T_{i}$ is defined as the sum of $T_{i}^{\text {diff }}$ from $\mathrm{r}_{\min }$ to $\mathrm{r}_{\max }$,

$$
\Delta T_{i}=\sum_{j=r_{\min }}^{r_{\max }} T_{i, j}^{\text {diff }}
$$

Where $r_{\min }=115 \mathrm{~mm}$ and $r_{\max }=125 \mathrm{~mm}$.

The maximum clarification $\Delta T_{\max }$ is defined as

$$
\Delta T_{\text {max }}=\left(\sum_{j=r_{\min }}^{r_{\max }} T_{e n d, j}-T_{1, j}\right) *\left(j_{r_{\text {max }}}-j_{r_{\text {min }}}\right)
$$

Where $T_{\text {end }}$ is the light transmission of the cell with water. In our case, $\bar{T}_{\text {end }}$ is 1 . 
The instability index is defined as the change of the clarification divided by the maximum possible clarification.

$$
\text { Instability index }=\frac{\Delta T_{i}}{\Delta T_{\max }}
$$

Batch Sedimentation Testing. The prepared particle suspensions were stored in glass vials with polypropylene caps. Subsequently, the vials were sealed with Parafilm to avoid evaporation and water absorption. The glass vials were stored in a fume hood and recorded with a surveillance camera at $24 \mathrm{fps}$ to observe the sedimentation behavior of the prepared suspensions under standard gravity for 90 days.

Analysis of Supernatants. The supernatants from the SiNP/ethylene glycol and SiNP/diethylene glycol suspensions were collected and filtered with a $200 \mathrm{~nm}$ glass filter (Merck Millipore, US) to remove the suspended SiNPs. Then, the supernatants were analyzed with ATR FTIR spectroscopy as described above. The supernatants were diluted with Mili-Q water $1: 1 \mathrm{v} / \mathrm{v}$. The $\mathrm{pH}$ of the diluted supernatants was measured with a $\mathrm{pH}$ meter (S20 SevernEasy ${ }^{\mathrm{TM}} \mathrm{pH}$, METTLER TOLEDO, Columbus, Ohio, US). In addition, an ion chromatograph (ICS 90, Dionex/Thermo Fischer, Waltham, Massachusetts, US) equipped with CS 12A (cations) and AS 12A (anions) columns was used for the identification of degradation products in the supernatants. The eluent's working pressure was set to $\sim 2000 \mathrm{psi}$ with a flow rate of $0.5 \mathrm{ml} \mathrm{min}{ }^{-1}$. Before injection for ion chromatography, the supernatants were diluted 10 times with Milli-Q water to avoid overloading the columns. 


\subsection{Supporting Information}

Table S3.1 Physical properties of the solvents and SiNPs used in this study. Dynamic viscosity is the viscosity of a $0.5 \mathrm{wt} \%$ SiNPs suspension in the respective solvent.

\begin{tabular}{|c|c|c|c|c|}
\hline & $\begin{array}{l}\text { density } \\
\left(\mathrm{kg} \mathrm{m}^{-3}\right)\end{array}$ & $\begin{array}{c}\text { dielectric } \\
\text { constant }(303 k)\end{array}$ & $\begin{array}{c}\text { viscosity } \\
\text { (mpa) }\end{array}$ & $\begin{array}{c}\text { dynamic } \\
\text { visocosity (mpa) }\end{array}$ \\
\hline methanol & $792^{60}$ & $31.7^{60}$ & 0.6 & 1.7 \\
\hline ethanol & $789^{60}$ & $23.6^{60}$ & 1.2 & 2.5 \\
\hline 1-hexanol & $814^{61}$ & $12.7^{62}$ & 4.7 & 5.8 \\
\hline isobutanol & $802^{63}$ & $17.4^{64}$ & 4.0 & 5.1 \\
\hline ethylene glycol & $1110^{65}$ & $37.6^{65}$ & 16.3 & 16.8 \\
\hline diethylene glycol & $1120^{65}$ & $29.1^{65}$ & 21.8 & 22.4 \\
\hline SiNPs & 2328 & N/A & N/A & N/A \\
\hline
\end{tabular}

Table S3.2 The sedimentation velocity range of SiNPs under Earth's gravity (ve) field and $A C$ (vac); and the corresponding time for SiNPs to settle $1 \mathrm{~cm}$ te and tac. The minimum/maximum hydrodynamic diameter of SiNPs obtained by DLS measurements were used to calculate the range of $v$ and $t$. The parameters used to perform the calculation are shown in Table S3.1.

\begin{tabular}{|c|c|c|c|c|c|c|}
\hline & methanol & ethanol & $\begin{array}{c}\text { 1- } \\
\text { hexanol }\end{array}$ & isobutanol & $\begin{array}{c}\text { ethylene } \\
\text { glycol }\end{array}$ & $\begin{array}{l}\text { diethylene } \\
\text { glycol }\end{array}$ \\
\hline$v_{e}\left(n m s^{-1}\right)$ & $7.3-138.8$ & $5.0-94.6$ & $2.1-40.1$ & $2.4-46.0$ & $0.6-11.1$ & $0.5-8.3$ \\
\hline$t_{e}(h)$ & $20-379$ & $\begin{array}{l}29.4- \\
556.3\end{array}$ & $\begin{array}{c}69.2- \\
1311.8\end{array}$ & $60.4-1144.5$ & $\begin{array}{l}249.3- \\
4723.3\end{array}$ & $335.2-6349.8$ \\
\hline$v_{a c}\left(\mu m s^{-1}\right)$ & $9.5-180.5$ & $6.5-123$ & $2.8-52.1$ & $3.2-59.8$ & $0.8-14.5$ & $0.6-10.8$ \\
\hline$t_{a c}(\min )$ & $0.9-17.5$ & $1.4-25.7$ & $3.2-60.5$ & $2.8-52.8$ & $\begin{array}{c}11.5- \\
218\end{array}$ & $15.5-293.1$ \\
\hline
\end{tabular}

\subsection{References}

(1) Pellegrino, T.; Kudera, S.; Liedl, T.; Javier, A. M.; Manna, L.; Parak, W. J. On the Development of Colloidal Nanoparticles towards Multifunctional Structures and Their Possible Use for Biological Applications. Small 2005, 1 (1), 48-63.

(2) Lu, Z.; Yin, Y. Colloidal Nanoparticle Clusters: Functional Materials by Design. Chemical Society Reviews 2012, 41 (21), 6874-6887.

(3) Volokitin, Y.; Sinzig, J.; de Jongh, L. J.; Schmidt, G.; Vargaftik, M. N.; Moiseev, I. I. Quantum-Size Effects in the Thermodynamic Properties of Metallic 
Nanoparticles. Nature 1996, 384 (6610), 621-623.

(4) Wood, V.; Bulović, V. Colloidal Quantum Dot Light-Emitting Devices. Nano Reviews 2010, 1 (1), 5202.

(5) Lohse, S. E.; Murphy, C. J. Applications of Colloidal Inorganic Nanoparticles: From Medicine to Energy. Journal of the American Chemical Society 2012, 134 (38), 15607-15620.

(6) Talapin, D. v.; Lee, J. S.; Kovalenko, M. v.; Shevchenko, E. v. Prospects of Colloidal Nanocrystals for Electronic and Optoelectronic Applications. Chemical Reviews 2010, 110 (1), 389-458.

(7) Turkevich, J.; Stevenson, P. C.; Hillier, J. A Study of the Nucleation and Growth Processes in the Synthesis of Colloidal Gold. Discussions of the Faraday Society 1951, 11, 55-75.

(8) Stöber, W.; Fink, A.; Bohn, E. Controlled Growth of Monodisperse Silica Spheres in the Micron Size Range. Journal of Colloid And Interface Science 1968, 26 (1), 62-69.

(9) Stephen, P. S. (1965). Low Viscosity Magnetic Fluid Obtained By the Colloidal Suspension of Magnetic Particles (U.S. Patent No. 3215572). U.S. Patent and Trademark Office. https://patents.google.com/patent/US3215572A/en

(10) Wang, S. Q.; Tooley, I. R. Photoprotection in the Era of Nanotechnology. Seminars in cutaneous medicine and surgery 2011, 30 (4), 210-3.

(11) Wang, Y.; Xia, Y. Bottom-up and Top-down Approaches to the Synthesis of Monodispersed Spherical Colloids of Low Melting-Point Metals. Nano Letters 2004, 4 (10), 2047-2050.

(12) Sperling, R. A.; Parak, W. J. Surface Modification, Functionalization and Bioconjugation of Colloidal Inorganic Nanoparticles. Philosophical Transactions of the Royal Society A: Mathematical, Physical and Engineering Sciences 2010, 368 (1915), 1333-1383.

(13) Hofmann, R. J.; Vlatkovic, M.; Wiesbrock, F. Fifty Years of Hydrosilylation in Polymer Science: A Review of Current Trends of Low-Cost Transition-Metal and Metal-Free Catalysts, Non-Thermally Triggered Hydrosilylation Reactions, and Industrial Applications. Polymers 2017, 9 (10), 534.

(14) O'Farrell, N.; Houlton, A.; Horrocks, B. R. Silicon Nanoparticles: Applications in Cell Biology and Medicine. International Journal of Nanomedicine 2006, 1 (4), 451-472.

(15) Jalkanen, T.; Mäkilä, E.; Määttänen, A.; Tuura, J.; Kaasalainen, M.; Lehto, V. P.; Ihalainen, P.; Peltonen, J.; Salonen, J. Porous Silicon Micro- and Nanoparticles for Printed Humidity Sensors. Applied Physics Letters 2012, 101 (26), 263110.

(16) Lawes, S.; Sun, Q.; Lushington, A.; Xiao, B.; Liu, Y.; Sun, X. Inkjet-Printed Silicon as High Performance Anodes for Li-Ion Batteries. Nano Energy 2017, 36, 313-321.

(17) Zeng, M.; Zhang, Y. Colloidal Nanoparticle Inks for Printing Functional Devices: Emerging Trends and Future Prospects. Journal of Materials Chemistry A 2019, 7 (41), 23301-23336.

(18) Erogbogbo, F.; Liu, T.; Ramadurai, N.; Tuccarione, P.; Lai, L.; Swihart, M. T.; Prasad, P. N. Creating Ligand-Free Silicon Germanium Alloy Nanocrystal Inks. ACS Nano 2011, 5 (10), 7950-7959. 
(19) Wheeler, L. M.; Neale, N. R.; Chen, T.; Kortshagen, U. R. Hypervalent Surface Interactions for Colloidal Stability and Doping of Silicon Nanocrystals. Nature Communications 2013, 4 (5), 1-10.

(20) Lazzari, S.; Moscatelli, D.; Codari, F.; Salmona, M.; Morbidelli, M.; Diomede, L. Colloidal Stability of Polymeric Nanoparticles in Biological Fluids. Journal of Nanoparticle Research 2012, 14 (6), 920.

(21) Segets, D.; Marczak, R.; Schäfer, S.; Paula, C.; Gnichwitz, J. F.; Hirsch, A.; Peukert, W. Experimental and Theoretical Studies of the Colloidal Stability of Nanoparticles?A General Interpretation Based on Stability Maps. ACS Nano 2011, 5 (6), 4658-4669.

(22) Yu, W.; Xie, H. A Review on Nanofluids: Preparation, Stability Mechanisms, and Applications. Journal of Nanomaterials 2012, 2012, 435873.

(23) Pau, P. C. F.; Berg, J. O.; McMillan, W. G. Application of Stokes' Law to Ions in Aqueous Solution. Journal of Physical Chemistry 1990, 94 (6), 2671-2679.

(24) Cheng, P. Y.; Schachman, H. K. Studies on the Validity of the Einstein Viscosity Law and Stokes' Law of Sedimentation. Journal of Polymer Science 1955, 16 (81), 19-30.

(25) Moore, T. L.; Rodriguez-Lorenzo, L.; Hirsch, V.; Balog, S.; Urban, D.; Jud, C.; Rothen-Rutishauser, B.; Lattuada, M.; Petri-Fink, A. Nanoparticle Colloidal Stability in Cell Culture Media and Impact on Cellular Interactions. Chemical Society Reviews 2015, 44 (17), 6287-6305.

(26) Studart, A. R.; Amstad, E.; Gauckler, L. J. Colloidal Stabilization of Nanoparticles in Concentrated Suspensions. Langmuir 2007, 23 (3), 1081-1090.

(27) Schubert, J.; Chanana, M. Coating Matters: Review on Colloidal Stability of Nanoparticles with Biocompatible Coatings in Biological Media, Living Cells and Organisms. Current Medicinal Chemistry 2018, 25 (35), 4553-4586.

(28) Wiese, G. R.; Healy, T. W. Effect of Particle Size on Colloid Stability. Transactions of the Faraday Society 1970, 66 (0), 490-499.

(29) Verwey, E. J. W. Theory of the Stability of Lyophobic Colloids. Journal of Physical and Colloid Chemistry 1947, 51 (3), 631-636.

(30) Boström, M.; Williams, D. R. M.; Ninham, B. W. Specific Ion Effects: Why DLVO Theory Fails for Biology and Colloid Systems. Physical Review Letters 2001, 87 (16), 168103/1-168103/4.

(31) Liyanage, D. D.; Thamali, R. J. K. A.; Kumbalatara, A. A. K.; Weliwita, J. A.; Witharana, S. An Analysis of Nanoparticle Settling Times in Liquids. Journal of Nanomaterials 2016, 2016, 7061838.

(32) Ilyas, S. U.; Pendyala, R.; Marneni, N. Dispersion Behaviour and Agglomeration Effects of Zinc Oxide Nanoparticles in Ethanol-Water Mixtures. Materials Research Innovations 2014, 18, S6-179-S6-183.

(33) Alexander, C. M.; Dabrowiak, J. C.; Goodisman, J. Gravitational Sedimentation of Gold Nanoparticles. Journal of Colloid and Interface Science 2013, 396, 5362 .

(34) Kops-Werkhoven, M. M.; Fijnaut, H. M. Dynamic Light Scattering and Sedimentation Experiments on Silica Dispersions at Finite Concentrations. The Journal of Chemical Physics 1980, 74 (3), 1618-1625.

(35) Furst, A.; Taulbee, D. N. (2005). Centrifugation - Analytical Ultracentrifugation. 
In P. Worsfold \& A. Townshend \& C. Poole (Ed.). Encyclopedia of Analytical Science (Second Edition) (pp 469-481). Elsevier.

(36) Gross-Rother, J.; Herrmann, N.; Blech, M.; Pinnapireddy, S. R.; Garidel, P.; Bakowsky, U. The Application of STEP-Technology ${ }^{\circledR}$ for Particle and Protein Dispersion Detection Studies in Biopharmaceutical Research. International Journal of Pharmaceutics 2018, 543 (1-2), 257-268.

(37) Walter, J.; Thajudeen, T.; Süß, S.; Segets, D.; Peukert, W. New Possibilities of Accurate Particle Characterisation by Applying Direct Boundary Models to Analytical Centrifugation. Nanoscale 2015, 7 (15), 6574-6587.

(38) Detloff, T.; Sobisch, T.; Lerche, D. Particle Size Distribution by Space or Time Dependent Extinction Profiles Obtained by Analytical Centrifugation (Concentrated Systems). Powder Technology 2007, 174 (1-2), 50-55.

(39) Viera, G.; Mikikian, M.; Bertran, E.; Cabarrocas, P. R. I.; Boufendi, L. Atomic Structure of the Nanocrystalline Si Particles Appearing in Nanostructured Si Thin Films Produced in Low-Temperature Radiofrequency Plasmas. Journal of Applied Physics 2002, 92 (8), 4684.

(40) Altman, I. S.; Agranovski, I. E.; Choi, M. Mechanism of Nanoparticle Agglomeration during the Combustion Synthesis. Applied Physics Letters 2005, 87 (5), 53104.

(41) Sailor, M. J. (2017). Chemical Reactivity and Surface Chemistry of Porous Silicon. In L. Canham (Ed.). Handbook of Porous Silicon (pp 1-20). Springer, Cham.

(42) He, L.; Kurata, Y.; Inokuma, T.; Hasegawa, S. Analysis of SiH Vibrational Absorption in Amorphous SiOx:H $(0 \leq \mathrm{x} \leq 2.0)$ Alloys in Terms of a ChargeTransfer Model. Applied Physics Letters 1993, 63 (2), 162-164.

(43) Pi, X. D.; Gresback, R.; Liptak, R. W.; Campbell, S. A.; Kortshagen, U. Doping Efficiency, Dopant Location, and Oxidation of Si Nanocrystals. Applied Physics Letters 2008, 92 (12), 123102.

(44) Yu, B. C.; Hwa, Y.; Park, C. M.; Sohn, H. J. Reaction Mechanism and Enhancement of Cyclability of SiO Anodes by Surface Etching with $\mathrm{NaOH}$ for Li-Ion Batteries. Journal of Materials Chemistry A 2013, 1 (15), 4820-4825.

(45) Wagner, C. D.; Passoja, D. E.; Hillery, H. F.; Kinisky, T. G.; Six, H. A.; Jansen, W. T.; Taylor, J. A. Auger and Photoelectron Line Energy Relationships in Aluminum-Oxygen and Silicon-Oxygen Compounds. Journal of vacuum science \& technology 1982, 21 (4), 933-944.

(46) López, G. P.; Castner, D. G.; Ratner, B. D. XPS O $\mathrm{O}_{1 \mathrm{~s}}$ Binding Energies for Polymers Containing Hydroxyl, Ether, Ketone and Ester Groups. Surface and Interface Analysis 1991, 17 (5), 267-272.

(47) Butt, H. J. Measuring Electrostatic, Van Der Waals, and Hydration Forces in Electrolyte Solutions with an Atomic Force Microscope. Biophysical Journal 1991, 60 (6), 1438-1444.

(48) LIFSHITZ, E. M.; Hamermesh, M. The Theory of Molecular Attractive Forces between Solids. Perspectives in Theoretical Physics 1992, 2 (1), 329-349.

(49) Israelachvili, J. N. (2011). Van Der Waals Forces between Particles and Surfaces. In J. Israelachvili (Ed.). Intermolecular and Surface Forces (pp 253-289) Academic Press: San Diego. 
(50) R. Clausius. (1867). The Mechanical Theory of Heat. Cambridge Press.

(51) Richardson, J. F.; Harker, J. H.; Backhurst, J. R. (2002) Sedimentation. In J. F. Richardson \& J. H. Harker \& J. Backurst (Ed.). Chemical Engineering Series (pp 237-290). Butterworth-Heinemann: Oxford.

(52) D'Arrigo, J. (2011). Structure of Predominant Surfactant Components Stabilizing Natural Microbubbles. In J. S. D’Arrigo (Ed.). Stable Nanoemulsions: SelfAssembly in Nature and Nanomedicine (pp 107-112); Elsevier.

(53) Cheng, N.-S. Effect of Concentration on Settling Velocity of Sediment Particles. Journal of Hydraulic Engineering 1997, 123 (8), 728-731.

(54) Sobisch, T.; Lerche, D. Characterization of Nanostructuring Effects at Solid/Liquid Interfaces by Analytical Centrifugation. Tenside, Surfactants, Detergents 2002, 39 (6), 232-236.

(55) White, G. F.; Russell, N. J.; Tidswell, E. C. Bacterial Scission of Ether Bonds. Microbiological Reviews 1996, 60 (1), 216-232.

(56) Rossiter, W. J.; Godette, M.; Brown, P. W.; Galuk, K. G. An Investigation of the Degradation of Aqueous Ethylene Glycol and Propylene Glycol Solutions Using Ion Chromatography. Solar Energy Materials 1985, 11 (5-6), 455-467.

(57) Israelachvili, J. N. (2011). Electrostatic Forces between Surfaces in Liquids. In J. Israelachvili (Ed.). Intermolecular and Surface Forces (pp 291-340). Academic Press: San Diego.

(58) Hülser, T.; Schnurre, S. M.; Wiggers, H.; Schulz, C. Gas-Phase Synthesis of Nanoscale Silicon as an Economical Route towards Sustainable Energy Technology. KONA Powder and Particle Journal 2011, 29 (29), 191-207.

(59) T. Detloff. Instability Index Calculation. 2012, 4 (2013), 1-4.

(60) González, B.; Calvar, N.; Gómez, E.; Domínguez, Á. Density, Dynamic Viscosity, and Derived Properties of Binary Mixtures of Methanol or Ethanol with Water, Ethyl Acetate, and Methyl Acetate at $\mathrm{T}=(293.15,298.15$, and 303.15) K. Journal of Chemical Thermodynamics 2007, 39 (12), 1578-1588.

(61) O'Neil, M. J. (2013). The Merck Index: An Encyclopedia of Chemicals, Drugs and Biologicals (15 th Edition). Royal Society of Chemistry, Cambridge.

(62) Elangovan, S.; Diriba Garbi, T.; Kena Etana, S. Dielectric Relaxation Studies Between Brompheniramine with 1-Butanol, 1-Pentanol and 1-Hexanol at 303K. Material Science Research India 2020, 17 (3), 230-235.

(63) Rumble, J. R. (2021). CRC Handbook of Chemistry and Physics. CRC Press.

(64) Rizk, H. A.; Elanwar, I. M. Dipole Moments of Glycerol, Isopropyl Alcohol, and Isobutyl Alcohol. Canadian Journal of Chemistry 1968, 46 (4), 10.1139/v68-084.

(65) Awwad, A. M.; Al-Dujaili, A. H.; Salman, H. E. Relative Permittivities, Densities, and Refractive Indices of the Binary Mixtures of Sulfolane with Ethylene Glycol, Diethylene Glycol, and Poly(Ethylene Glycol) at 303.15 K. Journal of Chemical and Engineering Data 2002, 47 (3), 421-424. 



\section{Chapter \\ 4}

\section{Polymer Brush Stabilized Silicon Nanoparticle Inks for Printed electronics: Effect on Colloidal Stability, Printability, and Diode Functionality}

Formulating stable nanoparticle-based conductive inks is a crucial step for successfully utilizing printing technologies in the low-cost, large-scale manufacturing of thin-film (electronic) devices (TFD). During ink formulation, both the long-term stability and printability of the ink need to be carefully adjusted. In this Chapter we present an approach to tackle both issues for the development of silicon nanoparticle-based inks by employing polymer brush coatings based on poly(methyl methacrylate) and poly(ethylene glycol) methacrylate. The polymer-coated nanoparticles show increased colloidal stability when using ethylene glycol and diethylene glycol as solvents. The printability of the polymer decorated silicon nanoparticle-based inks display improvements, especially nozzle clogging is significantly reduced. Finally, we demonstrate that the polymer coating negatively affects the device performance of printed silicon-based TFD. This we attribute to the inclusion of conductive SiC layers that form during device manufacturing when a polymer brush coating is present on the silicon nanoparticles. We suggest several strategies to avoid the formation of the SiC layers for future work.

Results described in this Chapter from parts of a (confidential) project "DruIDe" funded by the Interreg V project. 


\subsection{Introduction}

Silicon-based thin films are essential components in electronic devices used in our daily lives, e.g. in transistors or solar cells. ${ }^{1,2}$ With significant advances in nanotechnology, new avenues to fabricate devices with silicon-based thin films as functional components have become available. ${ }^{3,4}$ The use of well-established solutionbased high throughput printing methods to deposit silicon nanoparticle inks in a spatially controlled manner is considered the first step towards producing thin film (electronic) devices (TFD). Compared to conventional lithography, solution-based processing is advantageous due to its low cost and homogeneous layer formation, especially for large scale manufacturing of TFDs. ${ }^{5,6}$

Our research was part of a collaborative project, with the objective to supply our collaborators with printable Si nanoparticle containing, stable inks. The project partners (Duisburg University) were then supposed to use these inks in thin printed films and prepare elements for integrated circuits, such as diodes. Thus were interested in developing long-term stable silicon nanoparticle inks that enable the printing of silicon nanoparticle layers and their subsequent laser modification to fabricate functional Si $\mu$ cones layers in printed Schottky diodes on various substrates. At the start of this project, the silicon nanoparticle layers in proof-of-concept devices were prepared by spin coating, ${ }^{7}$ followed by laser treatment to form Si $\mu$-cones. The high crystallinity of the formed Si $\mu$-cones enabled a high operating frequency (switching speed up to $2.84 \mathrm{GHz}$ ) for the resulting Schottky diodes. Ultra-high-frequency (UHF) diodes are essential for applications like flexible targeted radio frequency identification (RFID) tags. To enable low-cost and large-scale manufacturing of flexible RFID tags with silicon nanoparticle inks, printing is considered as the processing technique of choice. So far, the RFID tags were built using laboratory processing techniques and the formulation and stability of silicon nanoparticle inks for printing have not been optimized yet.

Spin coating is considered the most straightforward solution processing technique for lab-scale research and development of thin films. ${ }^{8,9}$ Spin coating can produce layers with thicknesses from few nanometers to few micrometers by adjusting the solution viscosity, particle concentration, rotation velocity, and spinning duration. ${ }^{10,11}$ However, spin coating is not scalable to high throughput production and suffers from a high product to waste ratio (approximately $95 \%$ is waste). Therefore spin coating is considered unsuitable for large-scale manufacturing of thin film-based devices. ${ }^{12}$ 


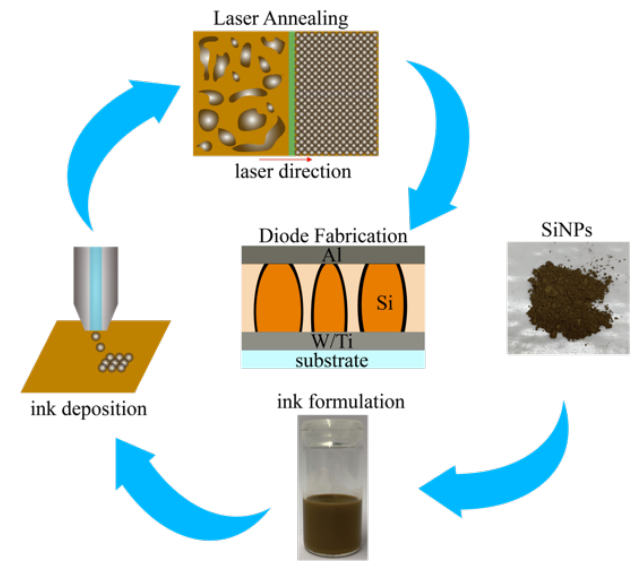

Figure 4.1 Schematic of SiNPs based Schottky diode manufacturing.

Essential for the successful transformation from spin coating to inkjet printing-based production approaches is the formulation of printable silicon nanoparticle-based inks that possess long-term stability. Several factors, like viscosity, density, surface tension, colloidal stability, will influence nanoparticle film formation and reproducibility. ${ }^{10,13}$ The project partners identified control over long-term colloidal stability (storage) to be essential to tackle early on prior to moving to industrial-scale production technologies. In our research we focused on two strategies: i) developing a broad understanding of silicon nanoparticles (SiNPs) colloidal stability in various solvents (see Chapter 3) and ii) utilizing steric stabilization to obtain long-term colloidal stability. The latter approach is presented and discussed in the remainder of this Chapter.

Steric stabilization of colloids can be achieved by various approaches, including surface charge stabilization mechanisms, and attachment of chain molecules (oligo- and polymers) that repel by an entropic spring effect. Here we focus on polymer chain attachment. Physical or covalent attachment of polymer chains to nanoparticle surfaces can create steric hindrance and eventually avoid particle agglomeration. ${ }^{14}$ In general, there are three ways to anchor polymer chains to nanoparticle surfaces, namely "grafting to", "grafting from", and "grafting through". ${ }^{15}$ Among these methods, "grafting from" by surface initiated-atom transfer radical polymerization (SI-ATRP) is recognized as a convenient approach for synthesizing polymer/particle hybrids which receives considerable attention (see also Chapter 2). ${ }^{16-18}$

Although colloidal stabilization by employing surface grafted polymers is a wellestablished technique, there are, to the best of our knowledge, little details in the literature to utilize SI-ATRP for enhancing colloidal stability of conductive nanoparticle inks for 
the printing of TFDs. Here we report on the preparation and use of the SI-ATRP based fabrication of PMMA/silicon and poly(ethylene glycol)methyl acrylate (PEGMA)/silicon nanoparticle hybrids and the effect of the polymer shell on the colloidal stability of their respective inks. In the last section of this Chapter, we discuss the effect of the polymer shell on the performance of a diode, followed by an outlook suggesting further developments.

\subsection{Results and Discussion}

\subsubsection{Printing Test of SiNP-Based Inks}

Using the colloidal stability data acquired from previous experiments (see Chapter 3), SiNP based inks were prepared by suspending $2 \mathrm{wt} \%$ of SiNPs in ethanol or diethylene glycol monobutyl ether (DGBE). Before printing, the inks were diluted to a particle concentration of $1 \mathrm{wt} \%$ using ethylene glycol (EG). The addition of EG increased the ink viscosity from $1.23 \mathrm{mPa}$ to $4.02 \mathrm{mPa}$ and from $6.25 \mathrm{mPa}$ to $17.2 \mathrm{mPa}$ for ethanol and DGBE based inks, respectively. This increase in ink viscosity was required to enhance the printability.

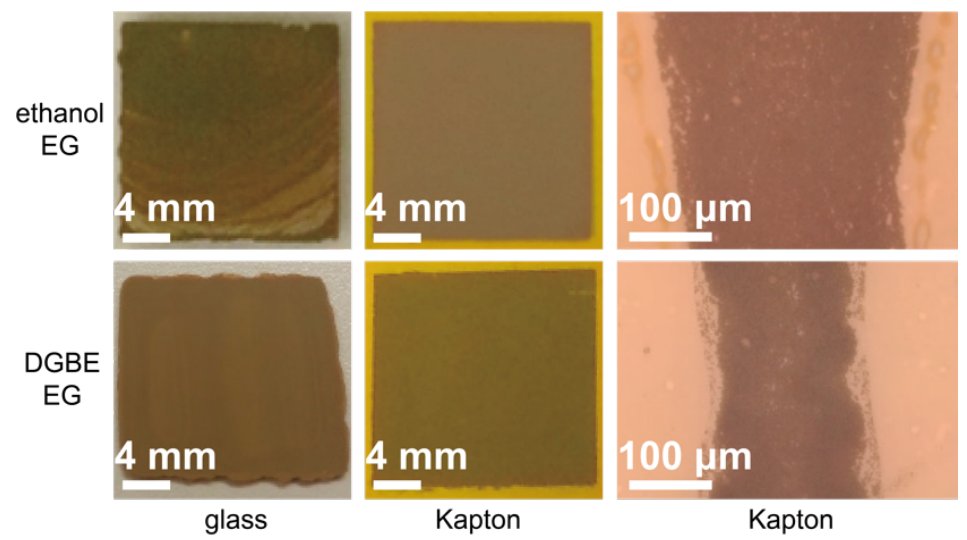

Figure 4.2. Images of inkjet-printed SiNP layers on glass (left) and Kapton (middle) substrates. On the right optical microscopy images of SiNP lines on Kapton are shown.

Images of printed SiNP layers are presented in Figure 4.2. When SiNP layers were printed on glass substrates, we see that for ethanol/EG as the ink solvent, the SiNP layer was non-uniform due to the coffee ring effect. When DEGB/EG was used as the solvent, the edges were distorted due to non-optimal ink wetting. Upon changing from glass substrates to Kapton substrates, the layer boundaries were much sharper, and the layers' interior appeared visually homogenous. However, numerous lighter spots inside the 100 
printed areas for both inks deposited on Kapton are visible in the optical microscopy images. These brighter spots could be the result of particle agglomeration upon ink deposition and/or solvent evaporation. Also, shortly after starting to print, the flowinduced aggregation of SiNPs at the contraction zone in the printhead caused $1 / 3$ of the nozzles to clog. This observed printer blocking is attributed to the broad size distribution of SiNPs used in this study (i.e. the hydrodynamic diameter in water ranges from $110 \mathrm{~nm}$ to $550 \mathrm{~nm}$ ). (We note that the SiNPs are aggregates consisting of primary particles with a diameter of $\sim 50 \pm 17 \mathrm{~nm}$. See Chapter 3 for more details about the SiNPs size distribution.) During printing, larger SiNP agglomerates act as heterogeneous nuclei for aggregation when passing the contraction zone and, consequently, induce nozzle clogging. ${ }^{19}$

Thus, our preliminary print tests suggested that pristine SiNPs are unsuitable for the inkjet printing of uniform thin SiNP films. To overcome irreversible particle clogging during printing and obtain more control over substrate wetting, we grafted PMMA and PEGMA from SiNPs.

\subsubsection{Surface Modification of SiNPs}

Thin polymers shells were grafted from the SiNP surface using SI-ATRP of MMA and EGMA. We selected PMMA and PEGMA to cover a range of suitable ink solvents, i.e. from polar to non-polar. The complete particle modification reaction scheme is presented in Figure 4.3. First, $\mathrm{HCl}$ was used to hydrolyze the SiNPs' surface to introduce surface-exposed silanol moieties, followed by their amino functionalization with (3Aminopropyl)triethoxysilane (APTES). Then the exposed amino functional groups on the particle surface were functionalized with $\alpha$-Bromoisobutyryl bromide (BiBB) to form SiNP based macroinitiators. Finally, PEGMA and PMMA brushes were grafted from the as-prepared nanoparticle macroinitiator surfaces via SI-ATRP of their respective monomers. 


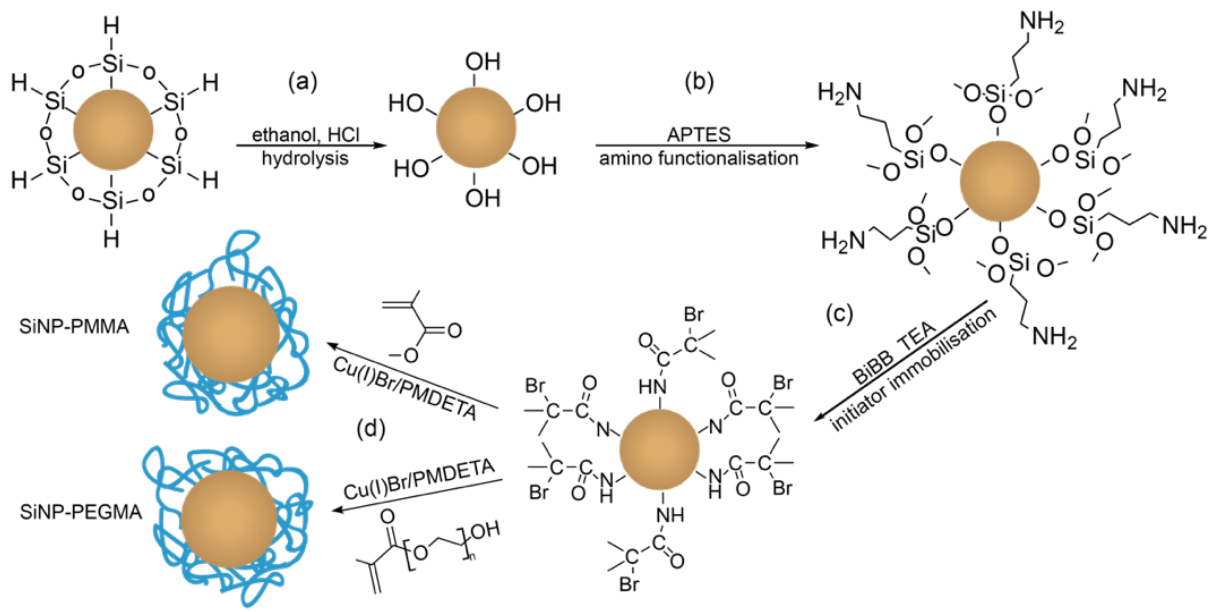

Figure 4.3 Schematic drawing of polymer grafting strategies. SiNPs were hydrolyzed (a) by diluted $\mathrm{HCl}$, followed by amino functionalization (b) to introduce amine groups to the particles' surface. Then, the bromine terminated ATRP initiator (BiBB) was covalently immobilized (c) on the SiNP surface. Subsequently, MMA and PEGMA monomers were grafted from the particles' surface (d) via SI-ATRP to form core-shell polymer/SiNP hybrids.

\subsubsection{Characterization of Polymer-Nanoparticle Hybrids}

Figure 4.4 shows transmission electron microscope (TEM) images of pristine SiNPs, PMMA grafted SiNPs (SiNPs-PMMA), and PEGMA grafted SiNPs (SiNPs-PEGMA). The TEM images show a core-shell structure for the polymer decorated SiNPs (Figure $4.4 \mathrm{~b}, 4.4 \mathrm{c}, 4.4 \mathrm{e}$, and $4.4 \mathrm{f}$ ). The dry shell thickness is approximately $1.8 \pm 0.5 \mathrm{~nm}$ and 1.7 $\pm 0.3 \mathrm{~nm}$ for the SiNPs-PMMA and SiNPs-PEGMA, respectively.

Next to TEM, Fourier transform infrared (FTIR) spectroscopy and thermogravimetric analysis (TGA) were employed to confirm the successful grafting of polymer shells on SiNPs. Figure 4.5 a shows single reflection FTIR absorbance spectra of the pristine SiNPs, SiNPs-PMMA, and SiNPs-PEGMA. The absorbance bands located at $1970 \mathrm{~cm}^{-1}, 2081 \mathrm{~cm}^{-1}, 2098 \mathrm{~cm}^{-1}$, and $2270 \mathrm{~cm}^{-1}$ in the FTIR spectrum of the SiNPs correspond to $\mathrm{Si}-\mathrm{H}$ stretching vibration of $\mathrm{H}-\mathrm{Si}\left(\mathrm{Si}_{3}\right), \mathrm{H}-\mathrm{Si}\left(\mathrm{Si}_{2} \mathrm{O}\right), \mathrm{H}-\mathrm{Si}\left(\mathrm{SiO}_{2}\right)$, and $\mathrm{H}-$ $\mathrm{Si}\left(\mathrm{SiO}_{3}\right)$, respectively. ${ }^{20,21}$ After polymer grafting, $\mathrm{Si}-\mathrm{H}$ absorbances remain visible in the FTIR spectra of SiNPs-PMMA and SiNPs-PEGMA. This indicates that a significant amount of the surface exposed $\mathrm{Si}-\mathrm{H}$ groups were preserved during the surface modification process. Furthermore, the bands observed at $1715 \mathrm{~cm}^{-1}$ and near $2900 \mathrm{~cm}^{-1}$ in the FTIR spectra of the core-shell SiNPs are ascribed to carbonyl $(\mathrm{C}=\mathrm{O})$ absorbances of the ester and $\mathrm{C}-\mathrm{H}$ absorbances in the grafted polymers, respectively. ${ }^{22}$ The appearance 102 
of the $\mathrm{C}-\mathrm{H}$ and $\mathrm{C}=\mathrm{O}$ absorbances in the FTIR spectra of the hybrid SiNPs confirm the successful grafting of PMMA and PEGMA shells.
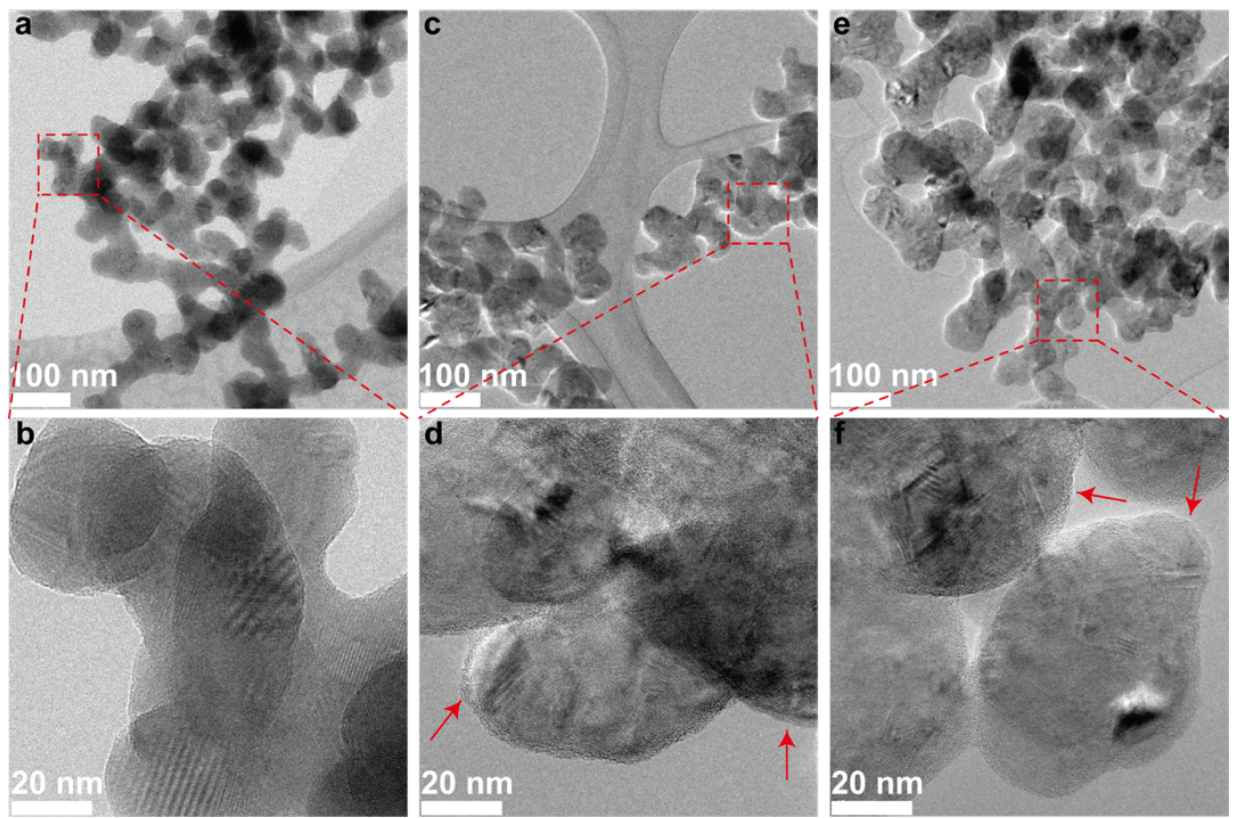

Figure 4.4 TEM images of pristine SiNPs ( $a$ and $d)$, SiNPs-PMMA ( $b$ and e), and SiNPsPEGMA ( $c$ and f). The red arrows point to the grafted polymer shells around the SiNPs.
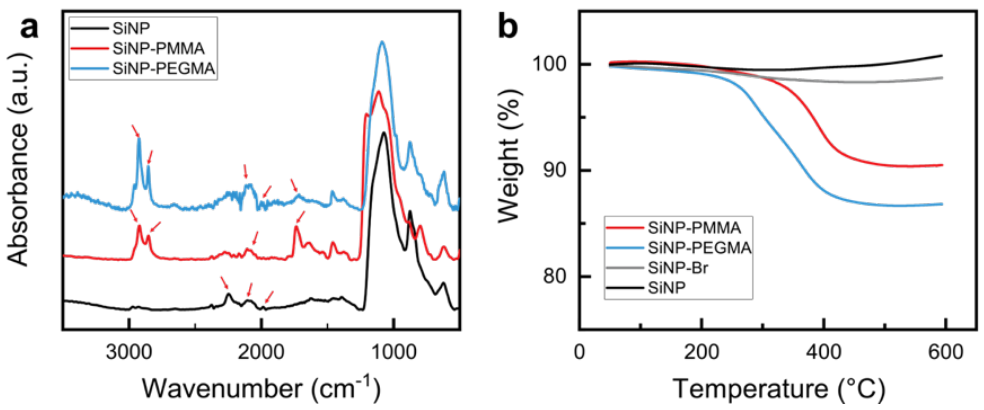

Figure 4.5 a) Single reflection FTIR absorption spectra of SiNPs, SiNPs-PMMA, and SiNPs-PEGMA. b) TGA thermograms for SiNPs, SiNPs-Br, SiNPs-PMMA, and SiNPsPEGMA.

TGA was used to determine the amount of grafted polymer to the SiNPs. The nonisothermal thermograms for SiNPs, initiator, immobilized (SiNPs-Br), and PMMA/PEGMA grafted SiNPs are shown in Figure 4.5b. From these thermograms, we determined that approximately $2.1 \mathrm{wt} \%$ of ATRP functional initiator was coupled to the surface of the SiNPs. The grafted amount of PMMA and PEGMA to SiNPs was 
approximately $8.2 \%$ and $11.9 \%$, respectively. Based on the shell thickness values determined by TEM analysis, the amounts of grafted polymers are in qualitative agreement with what we expected. Overall, we conclude that SiNPs-PMMA and SiNPsPEGMA were successfully prepared.

\subsubsection{Surface Modification of SiNPs}

Following SiNPs-PMMA and SiNPs-PEGMA preparation, SiNPs based inks were formulated with EG and DGBE as ink solvents. EG and DGBE were selected as a good starting point to develop the SiNPs hybrid inks due to their relatively high boiling point and suitable viscosity for inkjet printing. Both PMMA and PEGMA coating enhance the colloidal stability for SiNPs. In batch sedimentation evaluation, both SiNPs-PMMA and SiNPs-PEGMA remained dispersed in EG for more than 90 days. On the contrary pristine SiNPs revealed the first signs of particle sedimentation after 15 days already.

We adjusted the ink viscosity during printing trials by reducing the particle loading and/or mixing in various other solvents. Table 4.1 shows the composition of the optimized inks we prepared for the printing trials.

Table 4.1 The composition of SiNP, SiNPs-PMMA, and SiNPs-PEGMA based inks.

\begin{tabular}{cccc}
\hline & Ink 1 & Ink 2 & Ink 3 \\
Particle & SiNP & SiNPs-PMMA & SiNPs-PEGMA \\
Particle load & $4 \mathrm{wt} \%$ & $3 \mathrm{wt} \%$ & $5.5 \mathrm{wt} \%$ \\
Solvent & DGBE & EG/DCM/acetone $(2: 5: 3 \mathrm{v} / \mathrm{v})$ & DGBE
\end{tabular}

Contrary to SiNPs based inks (see section 3.1), the formulated hybrid SiNPs based inks enabled us to print patterns on titanium/tungsten coated glass substrates, as is shown in Figure 4.6, without significant nozzle clogging ( $<10 \%$ of the nozzles). In addition, when nozzles did clog, they were easily opened by wiping them clean with a tissue and modest rinsing. The reduced nozzle clogging was a significant improvement compared to printing with pristine SiNPs. Figure 4.6b, 4.6d, and 4.6f show confocal microscopy images of $10 \mathrm{pL}$ droplets printed in the drop-on-demand mode on titanium/tungsten coated glass. Table 4.2 presents the drop dimensions and coffee ring ratios for drops printed with the SiNPs, SiNPs-PMMA, and SiNPs-PEGMA based inks. 

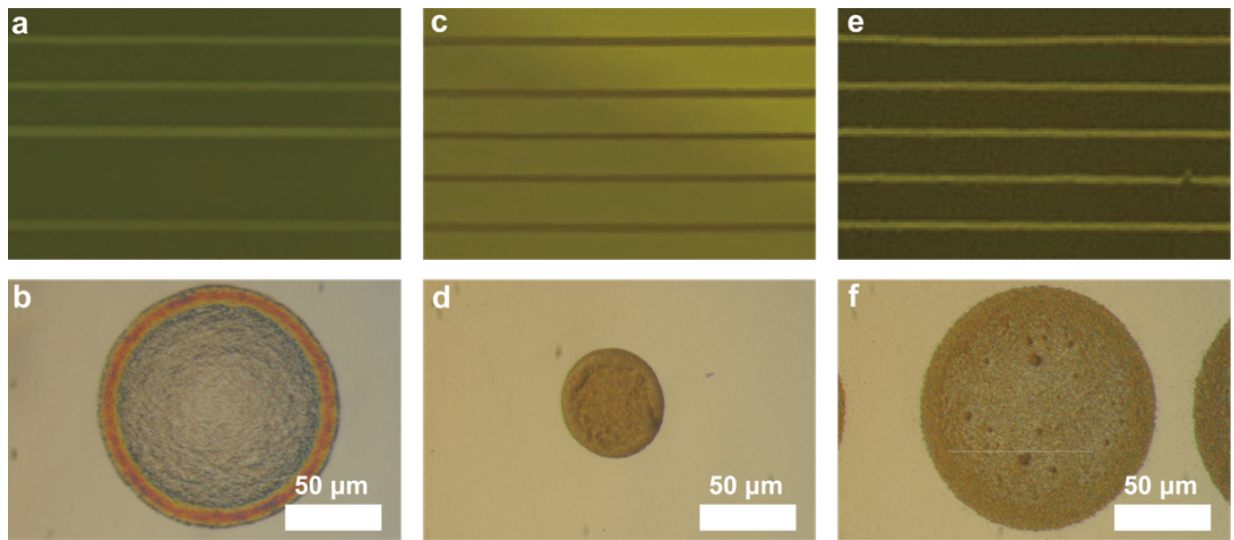

Figure 4.6. Photos of printed patterns (top) and confocal microscopy images (bottom) of drop-on-demand deposited SiNPs layers on titanium/tungsten coated glass substrates for SiNPs ( $a$ and b), SiNPs-PMMA ( $c$ and d), and SiNPs-PEGMA (e and f) based inks. The composition of the inks is shown in Table 4.1.

It is noteworthy to point out regarding data shown in Table 4.2 that printing with the SiNPs-PMMA based ink resulted in reduced droplet sizes and silicon layer thicknesses in the order of a few hundred nanometers. A decreased drop size and increase in drop thickness point at a better thin-film quality obtained by printing on the used titanium/tungsten coated glass substrates (compare Figure 4.6b, 4.6d, and 4.6f). In addition, the coffee ring ratio for the SiNPs-PMMA ink was reduced to $\sim 2.0$ as well. Considering the project objectives we note that the effect of the increased edge thickness compared to the interior of the printed droplets on the final quality of a printed silicon layer was not of primary interest. The project partners considered to elucidate the effect of a polymer shell around the SiNPs on $\mathrm{Si} \mu$-cone formation and Schottky diode performance of higher priority, which is presented in the next section of this Chapter.

Table 4.2 Droplet characterization for Ink 1, Ink 2, and Ink 3. The coffee ring ratio is defined as the ratio of the edge thickness to the interior drop thickness.

\begin{tabular}{cccc}
\hline & Ink 1 & Ink 2 & Ink 3 \\
Drop diameter (without edge) & $53 \mu \mathrm{m}$ & $43 \mu \mathrm{m}$ & $70 \mu \mathrm{m}$ \\
Drop thickness & $32 \mathrm{~nm}$ & $645 \mathrm{~nm}$ & $70 \mathrm{~nm}$ \\
Edge diameter & $126 \mu \mathrm{m}$ & $56 \mu \mathrm{m}$ & $122 \mu \mathrm{m}$ \\
Edge thickness & $95 \mathrm{~nm}$ & $1285 \mathrm{~nm}$ & $260 \mathrm{~nm}$ \\
Coffee ring ratio & 3.0 & 2.0 & 3.7
\end{tabular}




\subsubsection{Spin-Coating and Diode Fabrication}

To investigate the effect of a polymer shell around the SiNPs on deposited silicon layers, cone formation, and Schottky diode performance, our project partner at the University Duisburg-Essen, Prof. Bensons' team followed a reported ${ }^{23}$ protocol to construct thin-film diodes on titanium/tungsten coated glass substrates as a first step. This protocol utilizes spin-coating of SiNP based inks in ethanol with a relatively high particle loading, i.e. $16.6 \mathrm{wt} \%$, to obtain SiNP layers with a thickness of approximately $450 \mathrm{~nm}$. This thickness is required to have good Si $\mu$ cone formation during the laser modification step (See Figure 4.1).

SiNPs and SiNPs-PEGMA were dispersed in ethanol easily. For SiNPs-PMMA dispersions in the tri-solvent mixture, initial spin-coating trials revealed poor film formation, which we ascribed to the complex evaporation behavior of the solvent mixture. Few trials with various other solvents resulted in selecting DMF as a solvent for SiNPs-PMMA inks, giving visually homogeneous layers by spin-coating.
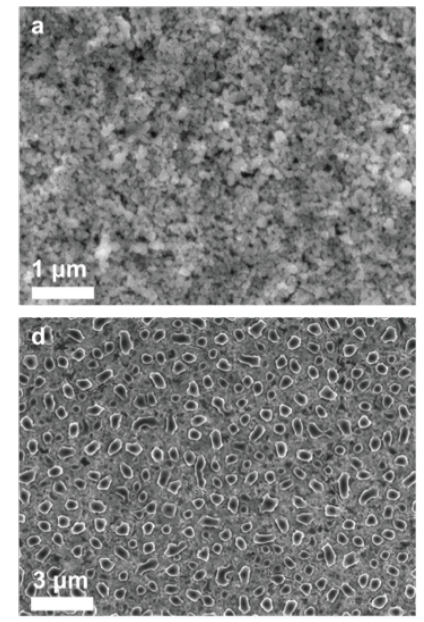
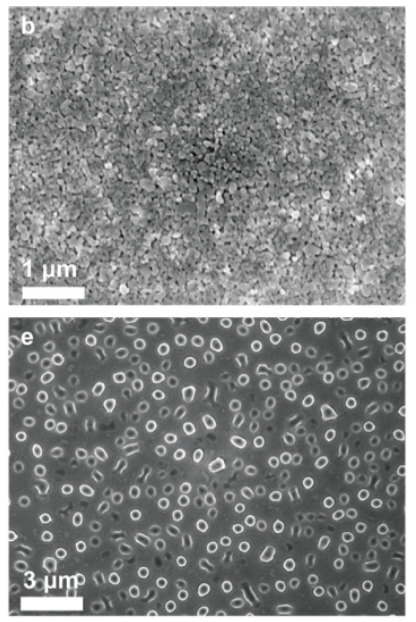
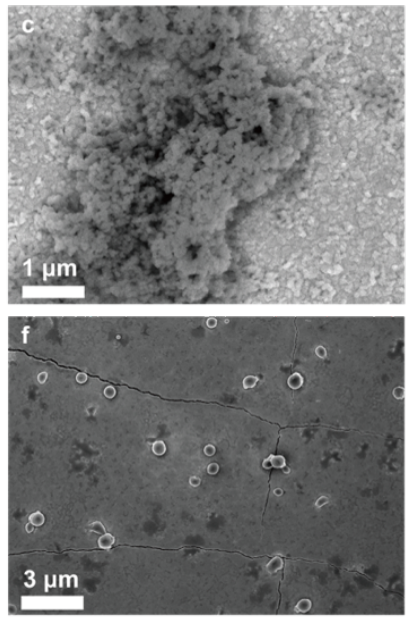

Figure 4.7. SEM images of a) SiNPs, b) SiNPs-PEGMA, and c) SiNPs-PMMA layers deposited on titanium/tungsten coated glass substrate via spin coating. SEM images of $S i$ $\mu$-cones fabricated from laser annealing of the d) SiNP, e) SiNPS-PEGMA, and f) SiNPsPMMA thin particle layers.

The as-prepared suspensions were deposited on the titanium/tungsten-coated glass substrates via spin coating. From the corresponding SEM images of the different systems shown in Figure 4.7, it is clear that SiNPs/ethanol and SiNPs-PEGMA/ethanol suspension formed smooth particle layers. The SiNPs-PMMA films after drying contained large particle agglomerates and the layers were not uniform. We attributed the formation of 
larger agglomerates to the relatively slow drying of the N,N-dimethylformamide-based (DMF-based) ink.

From the SEM images (Figure 4.7d-f), cone formation is observed for laser-treated SiNP, SiNPs-PEGMA, and SiNPs-PMMA layers. The values of the surface cone densities observed are $1.1 * 10^{6}, 1.1 * 10^{6}$ and $9.1 * 10^{4}$ cones $\mathrm{mm}^{-2}$, and the average cone widths are $430 \pm 60,470 \pm 50$, and $490 \pm 130 \mathrm{~nm}$ for the treated SiNP, SiNPs-PEGMA, and SiNPs-PMMA layers, respectively. The cone density and width for SiNPs-PEGMA are in the same order as values of the bare SiNP based $\mu$-cone layers. The Si $\mu$-cones formed for the SiNPs-PMMA layers have a much lower surface density, and cracks in the layer are visible. These observations are attributed to the melting of the larger agglomerates, resulting in forming of a thick silicon layer instead of Si $\mu$-cones, which, upon cooling due to thermal expansion differences between the coating and substrate, result in crack formation. Therefore only the SiNPs and SiNPs-PEGMA based layers were used to construct Schottky diodes.

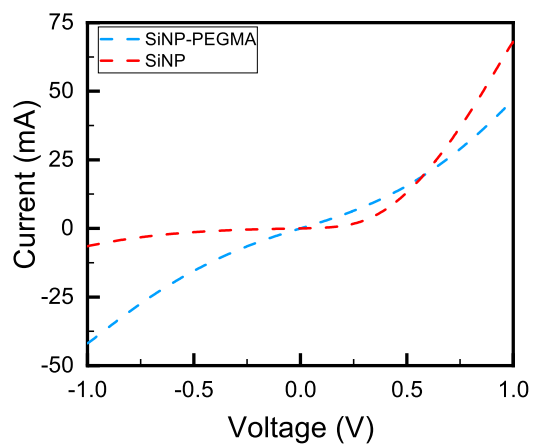

Figure 4.8. I-V characteristic of prepared Schottky diodes from SiNPs (red) and SiNPsPEGMA (blue) spin-coated layers

Figure 4.8 shows representative I-V (current-voltage) characteristics of devices build with SiNPs and SiNPs-PEGMA particle layers. The rectification ratios were 10.5 and 1.1 (at $|\mathrm{V}|=1 \mathrm{~V}$ ) for diodes constructed with Si $\mu$-cones based on SiNPs and SiNPs-PEGMA, respectively. The low rectification ratio for the diode based on the hybrid silicon particles means that we can hardly classify these devices as a diode. Below we provide a possible explanation for the low rectification ratio of the polymer grafted SiNPs based diodes.

X-ray photoelectron spectroscopy (XPS) analysis of the particle-based thin films and the $\mathrm{Si} \mu$-cones were used to obtain their composition and identify possible contaminants that could explain the poor rectification behavior of the SiNPs-PEGMA $\mu$-cone based diodes. Table 4.3 presents the atomic concentration of C, O, and Si for SiNP and SiNPs- 
PEGMA thin films together with their corresponding $\mu$-cone layer. It is readily observed that the carbon content increased from $11.8 \%$ to $30.3 \%$ for Si $\mu$-cone after PEGMA grafting. A large amount of $\mathrm{C}$ presented in the SiNPs-PEGMA $\mu$-cone could form nitrogen doped silicon carbide, resulting in poor rectification behavior. To further investigate the poor rectification behavior, $\mathrm{Si} 2 \mathrm{p}$ core-level spectra were measured for all samples as well. These are shown in Figure 4.9.

For the as-deposited SiNP and SiNPs-PEGMA layers, the dominant bands in the Si2p core-level spectra (Figure 4.9a and 4.9c) are ascribed to the characteristic binding energy for monoatomic $\mathrm{Si}(97.7 \mathrm{eV}$ and $98.9 \mathrm{eV}), \mathrm{Si}^{2+}(101.2 \mathrm{eV})$, and $\mathrm{Si}^{3+}(102.6 \mathrm{eV}){ }^{24,25}$ The $\mathrm{Si}^{2+}$ and $\mathrm{Si}^{3+}$ are most likely originating from the oxidized surface of the silicon nanoparticles ( $\mathrm{SiO}$ and $\mathrm{Si}_{2} \mathrm{O}_{3}$, respectively). Following laser modification, the dominant $\mathrm{Si} 2 \mathrm{p}$ bands for SiNP $\mu$-cone (Figure $4.9 \mathrm{~b}$ ) are $\mathrm{Si}^{1+}\left(\mathrm{Si}-\mathrm{OH}\right.$ and $\mathrm{Si}_{2} \mathrm{O}, 100.4 \mathrm{eV}$ ) and $\mathrm{Si}^{4+}$ $\left(\mathrm{SiO}_{2}, 103.5 \mathrm{eV}\right)$, indicating that elemental $\mathrm{Si}$ was oxidized. ${ }^{24}$ The peak located at 100.9 $\mathrm{eV}$ in the Si2p core-level spectrum of SiNPs-PEGMA $\mu$-cones (Figure 4.9d) is ascribed to $\mathrm{Si}-\mathrm{C}$, which is not observed in any other samples. ${ }^{25,26}$ The formation of $\mathrm{Si}-\mathrm{C}$ is explained by $\mathrm{C}$ atoms (from the polymer shell) bonding with $\mathrm{Si}$ atoms to form silicon carbide during the carbonization of the polymer chains. ${ }^{27}$

Table 4.3 Atomic concentrations of C, O, and Si for SiNP thin film, SiNP $\mu$-cone, SiNPsPEGMA thin film and SiNPs-PEGMA $\mu$-cone from XPS analysis.

\begin{tabular}{ccccc}
\hline & SiNP film & SiNP $\mu$-cone & SiNPs-PEGMA film & SiNPs-PEGMA $\mu$-cone \\
$\boldsymbol{C}$ & $13 \%$ & $11.8 \%$ & $55.9 \%$ & $30.3 \%$ \\
$\boldsymbol{O}$ & $40.3 \%$ & $52.7 \%$ & $35 \%$ & $45.3 \%$ \\
$\mathrm{Si}$ & $46.8 \%$ & $33.6 \%$ & $9.1 \%$ & $23.4 \%$
\end{tabular}

Furthermore, we speculate that the SiC impurities present on the $\mathrm{Si} \mu$-cones surfaces can interact with nitrogen during diode fabrication, resulting in nitrogen-doped silicon carbide. ${ }^{28}$ Nitrogen doping typically increases silicon carbide conductivity, leading to a metallic behavior instead of the needed semiconductive behavior of the formed Si $\mu$ cones. This can explain the low rectification ratio of the diodes based on SiNPs-PEGMA layers. 

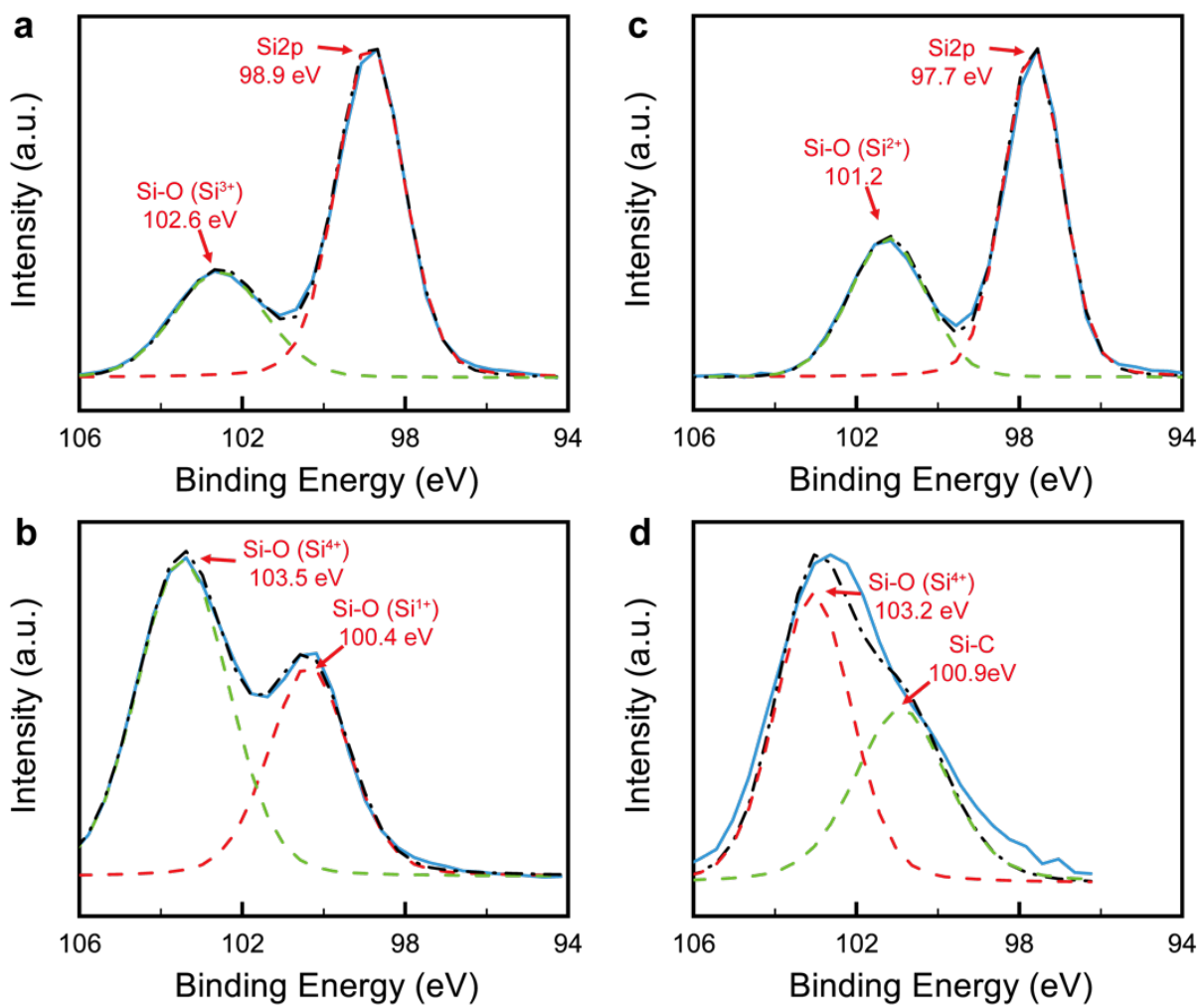

Figure 4.9. Si2p core-level spectra of SiNP thin film (a), SiNP $\mu$-cone (b), SiNPs-PEGMA thin film (c) and SiNPs-PEGMA $\mu$-cone (d). The dotted lines show peak deconvolution of the core-level spectra to identify the bonds present.

To minimize the influence of polymer grafts on the diode performance, we need to reduce the $\mathrm{C}$ content during diode fabrication. Using polymers that rapidly depolymerize into volatile small molecules upon certain triggering events can effectively reduce the $\mathrm{C}$ content. Polymers to consider are poly(isobutylene) and poly( $\alpha$-methylstyrene). Moreover, the molecules used to attach the surface-active ATRP initiators (APTES and $\mathrm{BiBB}$ ) contain a large amount of $\mathrm{C}$ atoms, as well. This could be avoided by exploiting an alternative grafting approach with minimal use of carbon-containing coupling molecules. One of the promising directions for this future work would be to use earthabundant species catalyzed or catalyst-free hydrosilylation. ${ }^{29}$

Based on the results presented and discussed, we believe that inkjet printing of hybrid SiNPs based inks with an optimized brush coating is a feasible approach for the largescale manufacturing of flexible electronic devices, but further research and progress is needed. 


\subsection{Conclusion}

SiNPs-PMMA and SiNPs-PEGMA with a core-shell structure were synthesized via SI-ATRP of their respective monomers. The polymer brush coating reduced the coffee ring effect and nozzle clogging for inkjet printing of thin particle layers on various substrates. Following laser sintering, spin-coated PEGMA grafted SiNP layers were able to form substrates with a uniform $\mathrm{Si} \mu$-cone layer with a cone density of $1.1^{*} 10^{6}$ and an average cone size of $470 \pm 50 \mathrm{~nm}$. The cone density and cone size of SiNPs-PEGMA layers are comparable to that of Si $\mu$-cones prepared from pristine SiNP ink. However, the SiNPs-PEGMA $\mu$-cone-based diodes had a rectification factor of 1.1 (at $|\mathrm{V}|=1 \mathrm{~V}$ ), while for pristine SiNPs $\mu$-cone-based diodes, the rectification factor is 10.5. Based on XPS analysis of the formed $\mu$-cone layers, we attributed the low rectification factor for PEGMA modified SiNPs based $\mu$-cones to the formation of nitrogen-doped SiC during diode fabrication. The inclusion of $\mathrm{SiC}$ is expected to result in more metallic characteristics instead of semiconductor behavior for the prepared diodes. Though a working diode was not produced, we conclude that polymer grafting is a powerful tool to improve the printability and stability of SiNP inks for large-scale implementation in the production of TFD. Furthermore, we recommend that future research focuses on selecting alternative polymers and coupling strategies for SiNPs to avoid SiC formation in the laser ablation step.

\subsection{Materials and Methods}

Materials. Boron-doped silicon nanoparticles with a boron doping level of $5^{*} 10^{18}$ atoms $\mathrm{cm}^{-3}$ were a gift from University Duisburg-Essen, Germany, and they were used as received. Hereinafter, we refer to these nanoparticles to as SiNPs. Dichloromethane (DCM) $\geq 99.5 \%$ was purchased from VWR International (Germany). Copper(I) bromide (CuBr) 98\% and (3-aminopropyl)-triethoxysilane (APTES) 97\% were received from Aldrich (Milwaukee, WI, USA). Hydrofluoric acid (48\%) ( $\mathrm{HCl}), \alpha$-bromoisobutyryl bromide (BiBB) 98\%, methylmethacrylate (MMA), poly(ethylene glycol) methacrylate (Mn 500) (PEGMA500), copper (II) bromide $\geq 99 \%$, aluminum oxide (for chromatography), triethylamine (TEA), ethylene glycol (anhydrous, 99.8\%), and diethylene glycol (ReagentPlus, 99\%) were purchased from Sigma-Aldrich (St. Louis, MO, USA). Butylated hydroxytoluene (BHT) stabilized tetrahydrofuran (THF) for analysis, and N,N-dimethylformamide AR (DMF) were acquired from Biosolve (Valkenswaard, the Netherlands). N,N,N',N",N"-pentamethyldiethylenetriamine (PMDETA) 98\% was purchased from Acros Organics (Geel, Belgium). Acetic acid 
$\geq 99 \%$ and diethylene glycol monobutyl ether (DGBE) for synthesis was obtained from Merck (Darmstadt, Germany). Ethylene glycol (EG) was purchased from VWR (Radnor, PA, USA). MMA and PEGMA were run over an aluminum oxide column to remove the inhibitor. Copper (I) bromide was purified by stirring in glacial acetic acid, followed by filtration and drying in a vacuum oven overnight at $60{ }^{\circ} \mathrm{C}$. Milli-Q water was produced by a Millipore Synergy system (Billerica, MA, USA). Unless specified, all other chemicals were used as received.

Hydrolysis of Silicon Nanoparticles. $5 \mathrm{~g}$ SiNPs were suspended in a mixture of 680 $\mathrm{ml}$ Mili-Q water and $20 \mathrm{ml}$ ethanol in a $1 \mathrm{~L}$ round bottom flask using intense sonication for 1 hour. While stirring at $500 \mathrm{rpm}$ at room temperature, $\mathrm{HCl}$ was added dropwise to the suspension until the $\mathrm{pH}$ value of the suspension reached approximately 1 . Subsequently, the suspension was left to stir at room temperature for $16 \mathrm{~h}$, followed by centrifuging at 13,000 rpm for $30 \mathrm{~min}$ to collect the hydrolyzed SiNPs (SiNPs-OH). The SiNPs-OH were redispersed with ethanol and centrifuged. This washing process was repeated two more times.

Amino-Functionalization of Silicon Nanoparticles. The as-prepared SiNPs-OH were redispersed in $700 \mathrm{ml}$ Ethanol in a $1 \mathrm{~L}$ round bottom flask by sonication for 1 hour, followed by the addition of $30 \mathrm{ml}$ APTES. Next, the suspension was left to stir at 500 rpm at room temperature for 17 hours. The amino-functionalized SiNPs $\left(\mathrm{SiNPs}_{\mathrm{N} H} \mathrm{~N}_{2}\right)$ were collected by centrifugation at 13,000 for $30 \mathrm{~min}$, redispersed in ethanol, and centrifuged again. This washing process was repeated two more times and particles were immediately treated with an ATRP functional initiator in the next step.

ATRP Initiator Immobilization on Silicon Nanoparticles. Freshly prepared SiNPs- $\mathrm{NH}_{2}$ were dispersed in DMF and centrifuged at 13,000 rpm for $30 \mathrm{~min}$. Next, the collected SiNPs- $\mathrm{NH}_{2}$ were dispersed in $600 \mathrm{ml}$ DMF in a $1 \mathrm{~L}$ round bottom flask using intense sonication for 1 hour. The suspension was cooled in an ice bath to $0{ }^{\circ} \mathrm{C}$, followed by the dropwise addition of $45 \mathrm{ml}$ TEA and $15 \mathrm{ml} \mathrm{BiBB}$ for $30 \mathrm{~min}$ while stirring at 500 rpm. Subsequently, the mixture was left in the ice bath while stirring for 1 hour. The temperature of the reaction mixture was raised to room temperature and stirring continued for another 16 hours. Following the reaction, the particles were collected by centrifugation at 13,000 rpm for $30 \mathrm{~min}$. The collected initiator immobilized SiNPs (SiNPs-Br) were redispersed in ethanol and centrifuged again to remove unreacted TEA, $\mathrm{BiBB}$, and the salts formed by TEA and $\mathrm{HBr}$. This washing step was repeated two more times. Finally, the as-prepared SiNPs-Br were dispersed in DMF and centrifuged at 13,000 rpm for $30 \mathrm{~min}$. Samples were used immediately for SI-ATRP. 
SI-ATRP of PEGMA, or PMMA, from Initiator Immobilized Silicon Nanoparticles. As-prepared SiNPs-Br were dispersed in $400 \mathrm{ml} \mathrm{DMF}$ in a 1L round bottom flask by sonication for 1 hour. After sonication, $40 \mathrm{ml}$ PEGMA500 was added to the suspension. Simultaneously, $80 \mathrm{mg} \mathrm{CuBr}, 25 \mathrm{mg} \mathrm{CuBr}$, and $15 \mathrm{ml} \mathrm{DMF}$ were added to a $25 \mathrm{ml}$ round bottom flask (flask B). Both flasks were sealed with a rubber septum and bubbled with nitrogen for $30 \mathrm{~min}$. Next, $0.14 \mathrm{ml}$ PMDETA was added to the $\mathrm{CuBr} / \mathrm{CuBr}_{2}$ mixture, followed by 30 min nitrogen purging, after which the mixture in flask B was transferred to the flask containing the SiNPs-Br/PEGMA500 suspension. Subsequently, the reaction flask was submerged into a $70^{\circ} \mathrm{C}$ thermostated oil bath and stirred at $500 \mathrm{rpm}$ for 1 hour under a nitrogen atmosphere. The polymer-modified particles were collected by centrifugation at 13,000 rpm for $30 \mathrm{~min}$. Then, the collected PEGMA grafted SiNPs (SiNPs-PEGMA) were redispersed in DMF and centrifuged again. The washing process was repeated 2 more times. Finally, the as-prepared SiNPsPEGMA were dispersed in ethanol, then centrifuged at 13,000 for $30 \mathrm{~min}$. The collected SiNPs-PEGMA were stored in ethanol.

To prepare PMMA grafted SiNPs (SiNPs-PMMA), we used the same procedure, except that $42.65 \mathrm{ml}$ MMA, $455 \mathrm{mg} \mathrm{CuBr}, 304 \mathrm{mg} \mathrm{CuBr}_{2}$, and $0.946 \mathrm{ml}$ PMDETA were employed. In addition, the final solvent transfer of the SiNPs-PMMA was to DCM.

Batch Sedimentation Testing of Inks. SiNPs-PEGMA were suspended in ethylene glycol and diethylene glycol at a concentration of $0.5 \mathrm{wt} \%$, respectively. The suspensions were stored in glass vials with polypropylene caps. Subsequently, the vials were sealed with Parafilm to avoid evaporation and water absorption. The glass vials were stored in ambient conditions and recorded with a surveillance camera at $24 \mathrm{fps}$ to observe the sedimentation behavior of the suspensions.

Particle Characterization. Transmission Electron Microscopy (TEM) was performed by an FEI/Philips CM300 system (FEI, Eindhoven, the Netherlands). Few drops of diluted particle dispersion in ethanol were deposited on the carbon side of a carbon/copper grid (HC200-Cu) (EMS, Germany) before imaging. The typically used electron acceleration voltage was between 3 to $5 \mathrm{keV}$. Fourier Transform Infrared (FTIR) spectra of the pristine and modified SiNPs were collected with a Bruker ALPHA single attenuated total reflection (ATR) FTIR Spectrometer equipped with an ATR single reflection diamond crystal (Bruker Optic $\mathrm{GmbH}$, Ettlingen, Germany). The spectra were collected in the range of $400-4000 \mathrm{~cm}^{-1}$ (spectral resolution of $4 \mathrm{~cm}^{-1}, 512$ scans). Background spectra were recorded against air. A Malvern Zetasizer Nano-ZS (Malvern Instruments, Malvern, UK) was used to determine the values of the effective 
hydrodynamic radius of SiNPs dispersed in Mili-Q water. The weight loss of the pristine and modified SiNPs was monitored with a TGA400 (PerkinElmer, Inc., Waltham, MA, USA). A sample weighing $\sim 6-10 \mathrm{mg}$ was loaded into a platinum pan and set to $50^{\circ} \mathrm{C}$ to stabilize. Subsequently, the sample was heated to $800{ }^{\circ} \mathrm{C}$ at a heating rate of $10{ }^{\circ} \mathrm{C} / \mathrm{min}$

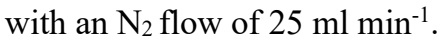

Inkjet Printing of Silicon Nanoparticles. A PiXDOR R\&D printer equipped with a $30 \mathrm{pL}$ printer head (128 nozzles) (Meyer Burger, Eindhoven, the Netherlands) was used to print SiNPs ethanol and DGBE dispersions. To prepare the SiNPs based inks, $0.2 \mathrm{~g}$ SiNPs were suspended in $4.9 \mathrm{~g}$ ethanol or DGBE via sonication (BRANSON 2510, Canada) for 1 hour. Following particle dispersion, $4.9 \mathrm{~g}$ of EG was added to the suspension, and the residual mixture was sonicated for 30 minutes. Then the as-prepared SiNPs inks were printed on glass and Kapton substrates. Polymer grafted SiNP based inks were printed with a Dimatix Piezo inkjet 2831 printer (FUJIFILM Dimatix Inc., Santa Clara, CA) PIXDRO LP 50, featuring a DMP-2850 print head and a DMC 11610 cartridge (16 nozzles having a $23 \mu \mathrm{m}$ diameter, $10 \mathrm{pL}$ nominal drop volume). Inks were prepared by suspending SiNPs or SiNPs-PEGMA in DGBE at a concentration of 4 and $5.5 \mathrm{wt} \%$, respectively, via sonication. SiNPs-PMMA were suspended by sonication in EG/DCM/acetone $(2: 5: 3 \mathrm{v} / \mathrm{v})$ or DCM at a concentration of $3.0 \mathrm{wt} \%$ and $2.0 \mathrm{wt} \%$, respectively. The as-prepared inks were printed on glass substrates $(15 \times 15 \mathrm{~mm})$ modified with sputter-deposited titanium and tungsten layers ( $1 \mu \mathrm{m}$ and $120 \mathrm{~nm}$, respectively).

Spin Coating of Polymer-Modified Silicon Nanoparticles. SiNPs-PEGMA and SiNPs-PMMA were suspended in ethanol and DCM at a concentration of $16.6 \mathrm{wt} \%$, respectively. The as-prepared suspensions were filtered with a $700 \mathrm{~nm}$ glass filter (Merck Millipore, US) before being cast on a titanium/tungsten coated glass substrate $(15 \times 15$ $\mathrm{mm}$ ), followed by spin coating at $6000 \mathrm{rpm}$ for $30 \mathrm{~s}$ (acceleration $6000 \mathrm{rpm} / \mathrm{s}$ ). To remove residual ethanol in the deposited polymer grafted SiNPs layers, spin-coated substrates were placed on a hotplate thermostated at $80{ }^{\circ} \mathrm{C}$ for 5 minutes. Images of the spin-coated thin films were obtained with an optical microscope (Olympus BH-2, Olympus, Tokyo, Japan) and a scanning electron microscope (JEOL Field Emission JSM-7500F, JEOL Benelux, Nieuw-Vennep, the Netherlands).

Schottky Diode Formation. As-deposited SiNP thin films were annealed with an ATLEX-300SI KrF excimer laser (ATL Lasertechnik GmbH, Wermelskirchen, Germany) to form $\mathrm{Si} \mu$-cones. The laser's emitting wavelength and pulse duration were $248 \mathrm{~nm}$ and 4-6 ns, respectively. The annealing process was performed under a nitrogen environment to avoid oxidation of Si-melt. During the process, the repetition frequency, 
the scanning speed, and the effective energy density for the laser were set to $100 \mathrm{~Hz}, 9.3$ $\mathrm{mm} \mathrm{min}^{-1}$ and $5 \mathrm{~mJ}$, respectively. SU-8 2000.5 was used as the insulator layer between anode and cathode. It is applied to cover the annealed Si $\mu$-cones on the sample via lithography, followed by $30 \mathrm{~s}$ plasma etching to expose the top of the $\mathrm{Si} \mu$-cones eventually. The as-prepared sample was dipped in $5 \%$ hydrofluoric acid solution for 20 $\mathrm{s}$ to remove the oxidized layer from the top of the Si $\mu$-cones. Then a layer of aluminum $(200 \mathrm{~nm})$ was deposited via evaporation to form Schottky contact. The IV characteristics of as-prepared diodes were measured with a KEITHLEY 4200-SCS (Keithley Instruments GmbH, Ohio, U.S.).

\subsection{References}

(1) Durrani, Z. A. K.,; Ahmed, H. (2008) Nanosilicon Single-Electron Transistors and Memory. In V. Kumar (Ed.). Nanosilicon (pp335-359). Elsevier.

(2) O'Farrell, N.; Houlton, A.; Horrocks, B. R. Silicon Nanoparticles: Applications in Cell Biology and Medicine. International Journal of Nanomedicine 2006, 1 (4), 451-472.

(3) Osborne, I.; Lavine, M.; Coontz, R. Materials for Electronics. Looking beyond Silicon. Introduction. Science 2010, 327 (5973), 1595.

(4) Venema, L. Silicon Electronics and Beyond. Nature 2011, 479 (7373), 309.

(5) Cummins, G.; Desmulliez, M. P. Y. Inkjet Printing of Conductive Materials: A Review. Circuit World 2012, 38 (4), 193-213.

(6) Yin, Z. P.; Huang, Y. A.; Bu, N. bin; Wang, X. M.; Xiong, Y. L. Inkjet Printing for Flexible Electronics: Materials, Processes and Equipments. Chinese Science Bulletin 2010, 55 (30), 3383-3407.

(7) Kühnel, L.; Neumann, K.; Neises, J.; Langer, F.; Erni, D.; Schmechel, R.; Benson, N. Nanoparticle Ink-based Silicon Schottky Diodes Operating up to $2.84 \mathrm{GHz}$. Nano Select 2020, 1 (6), 659-665.

(8) Kim, S. J.; Yoon, S.; Kim, H. J. Review of Solution-Processed Oxide Thin-Film Transistors. Japanese Journal of Applied Physics 2014, 53 (2 PART 2), 02BA02.

(9) Eslamian, M. Inorganic and Organic Solution-Processed Thin Film Devices. Nano-Micro Letters 2017, 9 (1), 1-23.

(10) Zabihi, F.; Xie, Y.; Gao, S.; Eslamian, M. Morphology, Conductivity, and Wetting Characteristics of PEDOT:PSS Thin Films Deposited by Spin and Spray Coating. Applied Surface Science 2015, 338, 163-177.

(11) Chaudhary, K. T. (2020). Thin Film Deposition: Solution Based Approach. In Thin Film. IntechOpen. doi:10.5772/intechopen.94455.

(12) Cui, Z.; Liao, L. (2020). Coating and Printing Processes. In C. Zheng (Ed.). Solution Processed Metal Oxide Thin Films for Electronic Applications (pp 8397). Elsevier.

(13) Nayak, L.; Mohanty, S.; Nayak, S. K.; Ramadoss, A. A Review on Inkjet Printing of Nanoparticle Inks for Flexible Electronics. Journal of Materials Chemistry C 2019, 7 (29), 8771-8795. 
(14) Lourenco, C.; Teixeira, M.; Simões, S.; Gaspar, R. Steric Stabilization of Nanoparticles: Size and Surface Properties. International Journal of Pharmaceutics 1996, 138 (1), 1-12.

(15) Zhao, B.; Brittain, W. J. Polymer Brushes: Surface-Immobilized Macromolecules. Progress in Polymer Science (Oxford) 2000, 25 (5), 677-710.

(16) Matyjaszewski, K. Atom Transfer Radical Polymerization (ATRP): Current Status and Future Perspectives. Macromolecules 2012, 45 (10), 4015-4039.

(17) Matyjaszewski, K. Advanced Materials by Atom Transfer Radical Polymerization. Advanced Materials 2018, 30 (23), 1706441.

(18) Matyjaszewski, K.; Xia, J. Atom Transfer Radical Polymerization. Chemical Reviews 2001, 101 (9), 2921-2990.

(19) Lee, A.; Sudau, K.; Ahn, K. H.; Lee, S. J.; Willenbacher, N. Optimization of Experimental Parameters to Suppress Nozzle Clogging in Inkjet Printing. Industrial and Engineering Chemistry Research 2012, 51 (40), 13195-13204.

(20) He, L.; Kurata, Y.; Inokuma, T.; Hasegawa, S. Analysis of SiH Vibrational Absorption in Amorphous $\mathrm{SiOx}: \mathrm{H}(0 \leq \mathrm{x} \leq 2.0)$ Alloys in Terms of a ChargeTransfer Model. Applied Physics Letters 1993, 63 (2), 162-164.

(21) San Andrés, E.; del Prado, A.; Mártil, I.; González-Díaz, G.; Bravo, D.; López, F. J.; Fernández, M.; Bohne, W.; Röhrich, J.; Selle, B.; Sieber, I. Bonding Configuration and Density of Defects of SiOxH y Thin Films Deposited by the Electron Cyclotron Resonance Plasma Method. Journal of Applied Physics 2003, 94 (12), 7462-7469.

(22) Han, S.; Kim, C.; Kwon, D. Thermal Degradation of Poly(Ethyleneglycol). Polymer Degradation and Stability 1995, 47 (2), 203-208.

(23) Kühnel, L.; Neumann, K.; Neises, J.; Langer, F.; Erni, D.; Schmechel, R.; Benson, N. Nanoparticle Ink-based Silicon Schottky Diodes Operating up to $2.84 \mathrm{GHz}$. Nano Select 2020, 1 (6), 659-665.

(24) Ma, J. W.; Lee, W. J.; Bae, J. M.; Jeong, K. S.; Oh, S. H.; Kim, J. H.; Kim, S. H.; Seo, J. H.; Ahn, J. P.; Kim, H.; Cho, M. H. Carrier Mobility Enhancement of Tensile Strained Si and SiGe Nanowires via Surface Defect Engineering. Nano Letters 2015, 15 (11), 7204-7210.

(25) Amjoud, M.; Reynes, A.; Morancho, R.; Carles, R. Comparative Study of Decomposition by Metal-Organic Chemical Vapour Deposition of Tetraethylsilane and Tetravinylsilane. Journal of Materials Chemistry 1992, 2 (11), 1205-1208.

(26) Ma, S. L.; Xu, B.; Xu, K. W.; Wu, X. L.; Chu, P. K. Annealing Behavior and Hardness Enhancement of Amorphous SiCN Thin Films. Journal of Vacuum Science \& Technology A: Vacuum, Surfaces, and Films 2007, 25 (5), 1407.

(27) Khashan, K. S.; Ismail, R. A.; Mahdi, R. O. Synthesis of SiC Nanoparticles by SHG $532 \mathrm{Nm}$ Nd:YAG Laser Ablation of Silicon in Ethanol. Applied Physics A: Materials Science and Processing 2018, 124 (6), 443.

(28) Sullivan, W.; Hettler, C.; Dickens, J. The Effects of Sub-Contact Nitrogen Doping on Silicon Carbide Photoconductive Semiconductor Switches. Proceedings of the 2012 IEEE International Power Modulator and High Voltage Conference, 2012, 77-79.

(29) Kuciński, K.; Hreczycho, G. Hydrosilylation and Hydroboration in a Sustainable 
Manner: From Earth-Abundant Catalysts to Catalyst-Free Solutions. Green Chemistry 2020, 22 (16), 5210-5224. 


\section{Chapter}

\section{Bubble Seeding Nanocavities: Multiple Polymer Foam Cell Nucleation by Polydimethylsiloxane Grafted Designer Silica Nanoparticles}

In this Chapter we describe a successful strategy to substantially enhance cell nucleation efficiency in polymer foams by using designer silica nanoparticles as nucleating agents. Bare and poly(dimethylsiloxane) (PDMS) grafted surface roughness engineered silica nanoparticles with diameters from $\sim 80 \mathrm{~nm}$ to $\sim 200 \mathrm{~nm}$ were synthesized and exploited as highly efficient cell nucleants in $\mathrm{CO}_{2}$-blown nanocellular poly(methyl methacrylate) (PMMA) foaming. The successful synthesis of core-shell nanoparticles was confirmed by Fourier transform infrared (FTIR) spectroscopy, thermogravimetric analysis (TGA), Brunauer-Emmett-Teller (BET) measurements, and transmission electron microscopy (TEM). The cell size and cell density of the PMMA micro- and nanocellular foams obtained were determined by scanning electron microscope (SEM). The results show that increased surface roughness enhances the nucleation efficiency of the designer silica particles. This is ascribed to a decreased nucleation free energy for foam cell nucleation in the nanocavities at the melt-nucleator interface. For PDMS grafted surface roughness engineered silica nanoparticles with a diameter of $155 \mathrm{~nm}$ and $200 \mathrm{~nm}$ multiple cell nucleation events were observed. These hybrid particles had nucleation efficiencies of 3.7 and 6.2, respectively. The surprising increase in nucleation efficiency to above unity is ascribed to the significant increase in $\mathrm{CO}_{2}$ absorption and capillary condensation in the corresponding PMMA during saturation. This results in the presence of large amounts of the physical blowing agent 
close to energetically favorable nucleating points. Additionally, it is shown that as a consequence of cell coalescence the increased number of foam cells is rapidly reduced during the first seconds of foaming. Hence, design of highly efficient nucleating particles, as well as a careful selection of foam matrix materials seem to be of pivotal importance to obtain polymer cellular materials with cell dimensions at the nanoscale. These findings contribute to the fabrication of polymer foams with high thermal insulation capacity, and have relevance in general for the area of cellular materials.

Parts of this Chapter has been published in: Liu, S.; Yin, S.; Duvigneau, J.; Vancso, G. J. Bubble Seeding Nanocavities: Multiple Polymer Foam Cell Nucleation by Polydimethylsiloxane-Grafted Designer Silica Nanoparticles. ACS Nano 2020, 14 (2), 1623-1634. Yin and Liu both contributed to the particle synthesis, corresponding composite preparation, and subsequent batch foaming of the samples. Yin focused more on the particle synthesis and Liu later focused more on the elucidation on the nanocavity induced multiple bubble seeding mechanism. 


\subsection{Introduction}

Nanocellular polymer foams are receiving considerable for a variety of reasons. ${ }^{1-6}$ The thermal conductivity of nanocellular foams is significantly reduced as a result of the Knudsen effect provided that the foam density is sufficiently low. ${ }^{3,7}$ In addition, the mechanical performance in terms of, e.g., the Young's modulus, impact properties and shore hardness is significantly improved compared to microcellular foams with similar foam densities. ${ }^{8-10}$ Thus nanocellular polymer foams with low thermal conductivity can offer great alternative options when considering new classes of materials for instance to achieve reductions in, for instant, fuel consumption and $\mathrm{CO}_{2}$ emissions in transportation as well as the thermal energy management of buildings.

Nanocellular polymer foam structures can be obtained by various strategies, selective extraction/decomposition, ${ }^{11,12}$ templating/imprinting, ${ }^{13-15}$ chemical/physical foaming, ${ }^{2,8,16-19}$ etc. Among these methods, $\mathrm{CO}_{2}$ based batch foaming is of particular interest, which is ascribed to good control over the foaming conditions, as well as to the low cost and environmental friendliness of $\mathrm{CO}_{2}$ as a physical blowing agent. ${ }^{10}$ However, nanocellular foams fabricated via batch foaming with high porosities $(>85 \%)$ and cell densities $\left(>10^{14}\right.$ cells $\left.\mathrm{cm}^{-3}\right)$ have rarely been reported. ${ }^{1,20-22}$ This is ascribed to the fact that it is difficult to nucleate the needed high cell densities in the early stages of foaming. In addition, on the timescale of foaming, cell coalescence is hard to avoid. Frequently used strategies to increase the nucleated cell density rely on increasing the physical blowing agent saturation pressure and/or increasing the pressure release rate. ${ }^{4,20,23}$ The introduction of nanostructured heterogeneous phases to polymers as heterogeneous nucleation sites prior to foaming has been considered a promising approach to obtainnanocellular foams. Examples utilized phase separated block copolymers ${ }^{24-27}$ and inorganic nanofillers as nucleating agents. ${ }^{6,17,28-32}$

$\mathrm{SiO}_{2}$ NPs are of particular interest to be employed as heterogeneous nucleation agents in polymer foaming because of their low cost, easy preparation and the simplicity of employing various surface decoration strategies. ${ }^{33,34}$ For instance, Zhai and coworkers ${ }^{30}$ demonstrated that the incorporation of silica nanoparticles $\left(\mathrm{SiO}_{2} \mathrm{NPs}\right)$ in polycarbonate prior to foaming resulted in a higher cell density and more uniform cell size distribution compared to the polycarbonate foams containing no $\mathrm{SiO}_{2} \mathrm{NPs}$ as nucleating agents. Yang and coworkers ${ }^{35}$ reported that the decoration of $\mathrm{SiO}_{2} \mathrm{NPs}$ with $\mathrm{CO}_{2}$-philic poly(ionic liquid) significantly enhances the cell nucleation in $\mathrm{CO}_{2}$ assisted polystyrene foaming compared to the polystyrene foams prepared with bare $\mathrm{SiO}_{2} \mathrm{NPs}$ as nucleating agents. Despite these promising results we note that the nanoparticles (NPs) utilized so far for 
nucleation suffered from low nucleation efficiencies, i.e. the number of foam cells obtained per number of NPs per $\mathrm{cm}^{3}$ added to the polymer matrix prior to foaming was small. Nucleation efficiencies for these inorganic particle based nanofillers are typically far below 0.01. ${ }^{17,35-37}$ This means that hundreds of added particles result in the formation of only 1 foam cell during foaming. In these calculations it is assumed that homogenous nucleation and cell coalescence can be neglected. ${ }^{4}$ Liu and coworkers ${ }^{28}$ reported on the successful utilization of poly(dimethylsiloxane) (PDMS) grafted $\mathrm{SiO}_{2} \mathrm{NPs}$ with a silica core diameter of $80 \mathrm{~nm}$ with a nucleation efficiency of approximately 0.5 for the preparation of $\mathrm{CO}_{2}$ batch blown poly(methyl methacrylate) (PMMA) foams. The good efficiency of these particles is ascribed to $i$ ) the low surface energy of the PDMS shell, which reduces the nucleation energy barrier, and $i$ ) the higher local $\mathrm{CO}_{2}$ concentration in the PDMS shell $(\sim 75 \mathrm{wt} \%)^{38}$ compared to the PMMA matrix ( $\left.18 \mathrm{wt} \%\right)^{39}$ Furthermore, they demonstrated that the contribution of line tension significantly reduces the nucleation efficiency of PDMS grafted NPs with a silica core diameter below $40 \mathrm{~nm}$ for the used foaming conditions (i.e. $\mathrm{CO}_{2}$ saturation pressure of 55 bar and foaming at 40 $\left.{ }^{\circ} \mathrm{C}\right) .{ }^{28}$ Thus the use of smaller particles, e.g., 10 nanometer in diameter, is considered unfavorable while it would in principle allow for the addition of the required high number of potential nucleation sites $\left(>10^{15} \mathrm{~cm}^{-3}\right)$ at relatively low weight percentages of particle loading. In fact, increasing the weight percentage of particle loading for particles with large enough diameters $(>60 \mathrm{~nm}$ ) to incorporate the required number of potential nucleation sites renders the resulting particle filled polymer matrix barely foamable.

Recently, Yang and coworkers ${ }^{40}$ established that cells nucleated from a concave surface have a lower nucleation free energy compared to the free energy of nucleation from flat or convex surfaces (e.g. spherical particles). ${ }^{41,42}$ Inspired by this work, we realized that the surface roughness is an often ignored, but important design parameter for nucleating hybrid particle designs to enhance nanocellular foaming.

One of the most commonly applied techniques to fabricate surface roughness enginnered silica nanoparticles $\left(\mathrm{SiO}_{2}-\mathrm{R}\right.$ NPs) includes attachment of NPs with substantially smaller radius. This method includes a two-step approach that relies on assembling smaller particles onto larger core particles via chemical or physical interactions, to result in the formation of $\mathrm{SiO}_{2}-\mathrm{R} \mathrm{NPs} .{ }^{43-46}$ By fine-tuning the diameter and concentration ratio of smaller and larger particles, NPs with a desired surface roughness values can be prepared. Puretskiy and coworkers ${ }^{46}$ succesfully exploited the attaching approach by first synthesizing poly(glycidyl methacrylate)-covered core $\mathrm{SiO}_{2}$ $\mathrm{NPs}$, followed by the attachment of amino-functionalized smaller $\mathrm{SiO}_{2} \mathrm{NPs}$. Wu and coworkers $^{45}$ reported covalently bonding $70 \mathrm{~nm}$ and $700 \mathrm{~nm} \mathrm{\textrm {SiO } _ { 2 }}$ NPs that were 
functionalized with amino and epoxy groups, respectively, resulting in $\mathrm{SiO}_{2}-\mathrm{R}$ NPs with a diameter of $850 \mathrm{~nm}$. Polymer particles have also been used as the core particles to prepare $\mathrm{SiO}_{2}-\mathrm{R}$ NPs with core-shell structure. ${ }^{47-49}$ For example, Xia et al. ${ }^{48}$ reported on the fabrication of poly(styrene)-silica core-shell particles via emulsion polymerization to form a polystyrene (PS) core followed by a sol-gel reaction to coat a rough silica shell. Instead of a sol-gel reaction to attach the silica shell, Wang and coworker ${ }^{47}$ used an attaching approach to achieve better surface topology. By covalently attaching smaller hydrolyzed $\mathrm{SiO}_{2}$ NPs onto poly(ethyleneglycol dimethacrylate-co-4-vinylpyridine) core particles, they fabricated surface roughened hybrid NPs.

Another possibility to obtain designer NPs with surface roughness control includes using etching method. Recently, Du and coworkers ${ }^{50}$ reported on the synthesis of $\mathrm{SiO}_{2}$ $\mathrm{R}$ NPs by etching of nearly spherical $\mathrm{SiO}_{2} \mathrm{NPs}$ with sodium borohydride $\left(\mathrm{NaBH}_{4}\right)$. The surface exposed $\mathrm{Si}-\mathrm{O}$ bonds dissolve at high $\mathrm{pH}$, resulting in the dissolution of silicate species. Upon supersaturation of the nanoparticle dispersion, silica domains are redeposited on the $\mathrm{SiO}_{2}$ NPs surface to form a rough surface. Compared to the above discussed attaching methods, the etching method offers a simpler approach for $\mathrm{SiO}_{2}-\mathrm{R}$ NPs fabrication.

In this Chapter we use both the attaching and the etching methods, respectively, to prepare surface roughness engineered $\mathrm{SiO}_{2} \mathrm{NPs}$ for their possible exploitation in a new application area, i.e. as foam cell nucleating agent in nanocellular foaming Of particular interest is to evaluate the nucleation efficiency of surface roughness engineered particles in the $\mathrm{CO}_{2}$ blown batch foaming of PMMA and compare the obtained nucleation efficiency values to various other silica nanoparticle based highly efficient nucleating particles.

We show that the $\mathrm{SiO}_{2}-\mathrm{R}$ NPs are efficient foam cell nucleators with nucleation efficiencies that are comparable to their PDMS grafted nearly spherical counterparts. Upon decorating the $\mathrm{SiO}_{2}-\mathrm{R}$ NPs with a few nanometer thick PDMS shell, multiple foam cell nucleation was observed from a single designer nanoparticle, and the value of highest nucleation efficiency obtained was 6.2 for particles with an overall core diameter of $200 \mathrm{~nm}$. This nucleation efficiency is nearly 40 times higher compared to that of a pristine, nearly spherical silica nanoparticle with a similar diameter. We show qualitatively that the high nucleation efficiency obtained for PDMS grafted $\mathrm{SiO}_{2}-\mathrm{R}$ NPs is ascribed to a significantly increased absorption of $\mathrm{CO}_{2}$ during saturation. This process results in a higher $\mathrm{CO}_{2}$ concentration close to the PDMS decorated, and contributes to forming energetically favorable nanocavities at the nucleating surface. The utilization of 
surface cavities combined with optimized surface chemistry for efficient heterogeneous nucleation at the macromolecular length scale is of interest for the production of nanocellular foams with high cell densities, and thus with superior thermal insulation properties.

\subsection{Results and Discussion}

\subsection{1 $\mathrm{SiO}_{2}-\mathrm{R}$ NPs Synthesis}

Two different methods, i.e., attaching and etching, were investigated as methods to control the surface roughness of $\mathrm{SiO}_{2} \mathrm{NPs}$. Both of these methods use Stöber $\mathrm{SiO}_{2} \mathrm{NPs}$ as the starting material(s).

Figure 5.1a depicts the reaction scheme for the attaching method to prepare $\mathrm{SiO}_{2}-\mathrm{R}$ NPs. First, the small $(80 \mathrm{~nm})$ and large $(600 \mathrm{~nm}) \mathrm{SiO}_{2} \mathrm{NPs}$ were functionalized with (3aminopropyl)-triethoxysilane (APTES) and (3-Glycidyloxypropyl)trimethoxysilane (GPS) to form amino- and epoxy- functionalized particles, respectively. Subsequently, the amino-functionalized $\mathrm{SiO}_{2} \mathrm{NPs}\left(\mathrm{SiO}_{2}-\mathrm{NH}_{2}\right)$ were covalently bonded to the epoxyfunctionalized $\mathrm{SiO}_{2} \mathrm{NPs}$ ( $\mathrm{SiO}_{2}$-GPS) by the ring-opening of the epoxy group under nitrogen atmosphere, resulting in the formation of $\mathrm{SiO}_{2}-\mathrm{R}$ NPs with a diameter of approximately $750 \mathrm{~nm}$. Figure $5.1 \mathrm{~b}$ shows a scanning electron microscope (SEM) image of $\mathrm{SiO}_{2}-\mathrm{R}$ NPs prepared by the attaching method. From the SEM image in Figure 5.1b it is readily observed that the smaller particles attached tend to form large clusters on the larger $\mathrm{SiO}_{2}$ NPs. Despite multiple attempts we did not obtain full surface coverage by using this approach. In addition, the multiple reaction steps, the relatively long reaction time, and the required dry atmosphere directed us towards using the etching method to produce surface roughened $\mathrm{SiO}_{2} \mathrm{NPs}$.
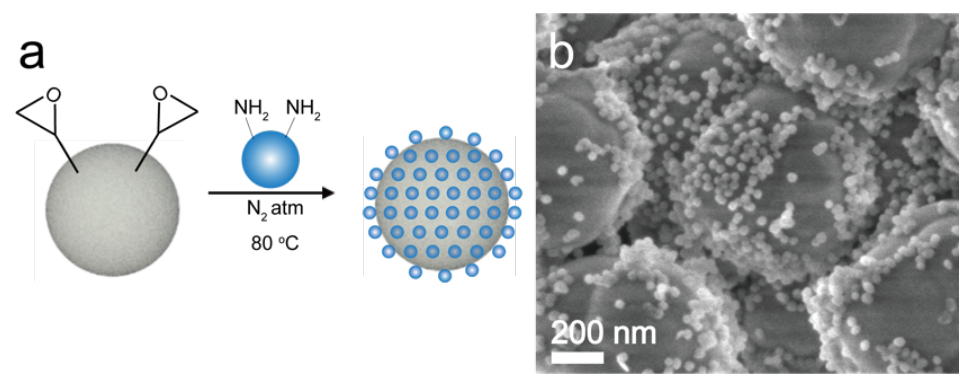

Figure 5.1 (a) Scheme of the $\mathrm{SiO}_{2}-\mathrm{R} N \mathrm{~N}$ preparation process via the attaching method. (b) SEM image of $\mathrm{SiO}_{2}-\mathrm{R} N \mathrm{NS}$ with a particle diameter of $\sim 750 \mathrm{~nm}$.

For the etching method, $\mathrm{NaBH}_{4}$ was used to adjust the $\mathrm{pH}$ of the $\mathrm{SiO}_{2}$ dispersion to 122 
eventually yield rough particle surfaces. The reaction scheme is depicted in Figure 5.2a. During the surface roughening reaction, the high basicity of the dissolved $\mathrm{NaBH}_{4}$ results in the dissociation of $\mathrm{Si}-\mathrm{O}$ bonds from the surface of nearly smooth $\mathrm{Stöber} \mathrm{SiO}_{2}\left(\mathrm{SiO}_{2}-\right.$ S) NPs and subsequently in the formation of soluble mono- and polysilicate species. Eventually the dissolved silicate species in the solution become supersaturated, which leads to the redeposition of small $\mathrm{SiO}_{2}$ NPs on the surface of the remaining silica NPs (Figure 5.2a, step 1)..$^{51-53}$

SEM images of $\mathrm{SiO}_{2}-\mathrm{S}$ NPs and $\mathrm{SiO}_{2}-\mathrm{R}$ NPs with a diameter of approximately 155 $\mathrm{nm}$ are shown in Figure 5.2b and c, respectively. The diameter of the $\mathrm{SiO}_{2}-\mathrm{R}$ is defined as the diameter of a circle just large enough to enclose the $\mathrm{SiO}_{2}-\mathrm{R}$ NPs outer surface. Particles with diameters between $80 \mathrm{~nm}$ and $200 \mathrm{~nm}$ were prepared (see Figure S5.1). Hereinafter, the numbers preceeding the name $\mathrm{SiO}_{2}$ refer to the apparent particle diameter. For instance, $155 \mathrm{SiO}_{2}-\mathrm{S}$ refers to an nanoparticle with an average diameter of $155 \mathrm{~nm}$.

From Figure 5.2c it is obvious that small silica domains (with diameters of $\sim 20$ to $30 \mathrm{~nm}$ ) are present at the surface of the NPs after treatment with $\mathrm{NaBH}_{4}$, which is in agreement with the etching and subsequent deposition of silicate species at the silica particle surface during the reaction. ${ }^{52}$

Following the successful synthesis of $\mathrm{SiO}_{2}$-R NPs with diameters of $\sim 200 \mathrm{~nm}, \sim 155$ $\mathrm{nm}, \sim 120 \mathrm{~nm}$ and $\sim 80 \mathrm{~nm}$, PDMS shells were grafted to each type, yielding the corresponding hybrid ( $\left.\mathrm{SiO}_{2}-\mathrm{RP}\right) \mathrm{NPs}$ (Figure 5.2a). In brief, $\mathrm{SiO}_{2}-\mathrm{R}$ NPs were derivatized with APTES, resulting in the formation of amine functionalized NPs $\left(\mathrm{SiO}_{2}-\mathrm{R}_{-} \mathrm{NH}_{2}\right)$ (step 2). The NPs were functionalized by PDMS employing the "grafting to" approach of monoglycidyl ether terminated PDMS (step 3). We note here that prolonged sonication or even melt blending did not destroy the surface roughness of the (hybrid) $\mathrm{SiO}_{2}-\mathrm{R}$ NPs i.e. the NPs are stable enough for utilization as nucleating particles in nanocellular foaming.

Figure 5.2d shows the Fourier transform infrared (FTIR) absorbance spectra of $\mathrm{SiO}_{2}$, $\mathrm{SiO}_{2}-\mathrm{R}, \mathrm{SiO}_{2}-\mathrm{R}-\mathrm{NH}_{2}, \mathrm{SiO}_{2}-\mathrm{RP}$ and nearly smooth PDMS grafted $\left(\mathrm{SiO}_{2}-\mathrm{SP}\right)$ NPs with a (silica core) diameter of $\sim 155 \mathrm{~nm}$. The remaining ethoxy groups after the Stöber reaction of tetraethyl orthosilicate (TEOS) are clearly observed in the FTIR spectra of the $155 \mathrm{SiO}_{2}-\mathrm{S}$ particles, i.e. the $\mathrm{CH}_{2} / \mathrm{CH}_{3}$ bending absorbance band at $1452 \mathrm{~cm}^{-1}$ and the $\mathrm{CH}_{2} / \mathrm{CH}_{3}$ absorbance band at $2980 \mathrm{~cm}^{-1} .^{54}$ After $\mathrm{NaBH}_{4}$ etching to yield surface roughened NPs these absorbance bands disappear, which indicates quantitative hydrolysis of the remaining ethoxy groups of the $155 \mathrm{SiO}_{2}-\mathrm{R} \mathrm{NPs}^{52}$ The reappearance of 
bands at $2980 \mathrm{~cm}^{-1}, 1450 \mathrm{~cm}^{-1}$ and $1380 \mathrm{~cm}^{-1}$ in the FTIR spectrum of $155 \mathrm{SiO}_{2}-\mathrm{R}-\mathrm{NH}_{2}$ are assigned to $\mathrm{CH}_{2}$ groups of the propyl spacer of surface tethered APTES. The presence of absorption bands ascribed to $\mathrm{CH}_{3}$ stretching at $2967 \mathrm{~cm}^{-1}$ and to $\mathrm{C}-\mathrm{H}$ bending at 1263 $\mathrm{cm}^{-1}$ in the FTIR spectrum of $155 \mathrm{SiO}_{2}-\mathrm{RP}$ NPs confirm the successful grafting of PDMS. ${ }^{55}$ The same absorbances are observed in the FTIR spectrum of $\mathrm{SiO}_{2}-\mathrm{SP}$ NPs; their synthesis is described in the experimental section.

a
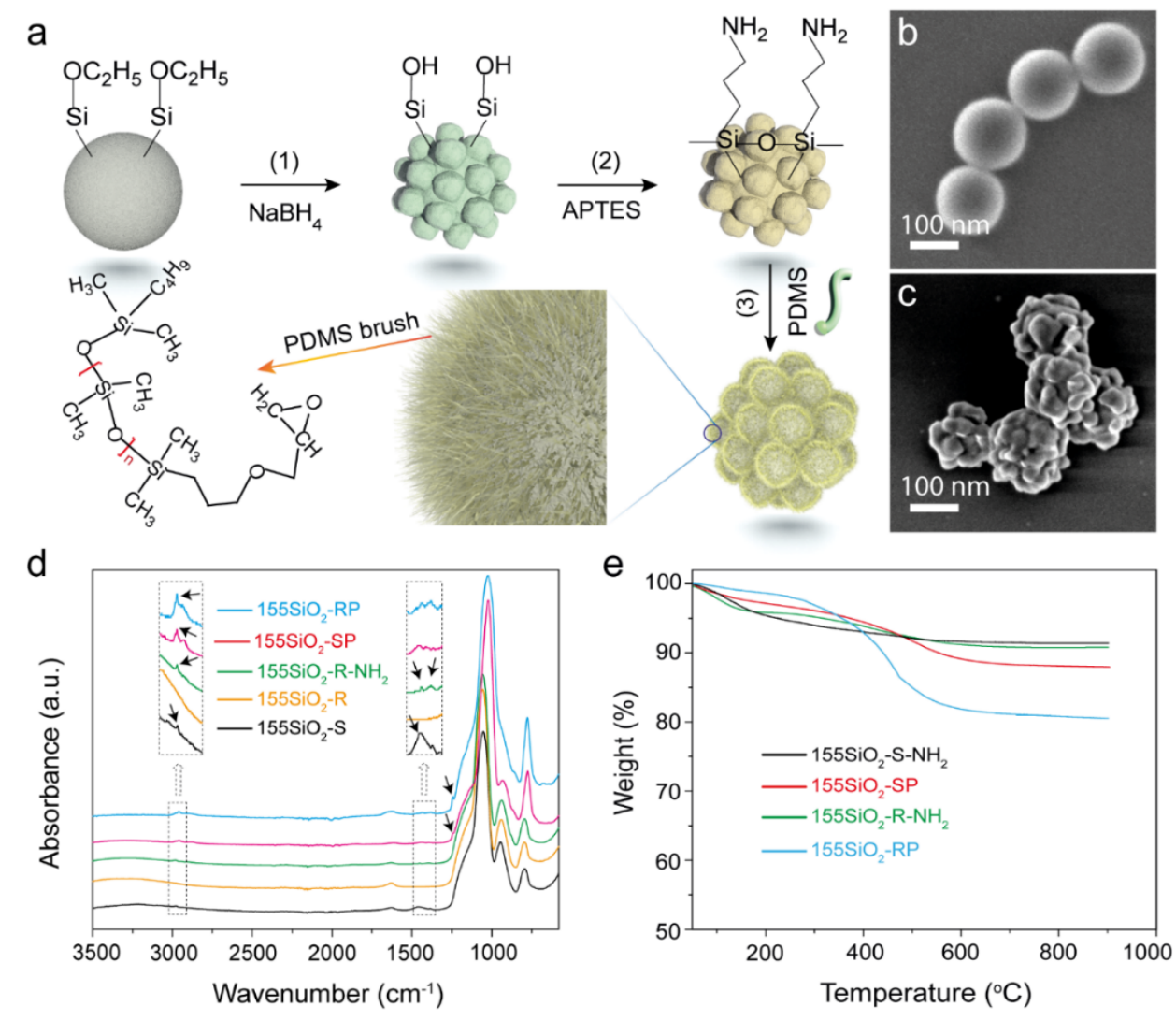

Figure 5.2 (a) A schematic of the $\mathrm{SiO}_{2}-\mathrm{RP}$ NPs preparation process. In (b) and (c) SEM images of $\mathrm{SiO}_{2}-\mathrm{S}$ and $\mathrm{SiO}_{2}-\mathrm{R} \mathrm{NPS}$ with a silica particle diameter of $\sim 155 \mathrm{~nm}$ are shown, respectively. (d) Single reflection ATR-FTIR absorbance spectra of $155 \mathrm{SiO}_{2}-\mathrm{S}, 155 \mathrm{SiO}_{2}-$ $\mathrm{R}, 155 \mathrm{SiO}_{2}-\mathrm{R}-\mathrm{NH}_{2}, 155 \mathrm{SiO}_{2}-\mathrm{RP}$ and $155 \mathrm{SiO}_{2}-\mathrm{SP} \mathrm{NPS}$. The black arrows in the FTIR spectra indicate characteristic FTIR absorbance values of the (modified) NPs. (e) Nonisothermal TGA thermograms of the $155 \mathrm{SiO}_{2}-\mathrm{S}-\mathrm{NH}_{2}, 155 \mathrm{SiO}_{2}-\mathrm{R}-\mathrm{NH}_{2}, 155 \mathrm{SiO}_{2}-\mathrm{SP}$ and $155 \mathrm{SiO}_{2}-\mathrm{RP} N P S$.

To determine the amount of PDMS grafted to the NPs, thermogravimetric analysis (TGA) was employed. Figure 5.2e shows the weight loss versus temperature curves for 
non-isothermal TGA measurements of $\mathrm{SiO}_{2} \mathrm{~S}-\mathrm{S}$ and $\mathrm{SiO}_{2}-\mathrm{R}$, amino-functionalized and PDMS grafted NPs with silica (core) diameters of approximately $155 \mathrm{~nm}$. The results clearly show that the amount of PDMS covalently bound to the $\mathrm{SiO}_{2} \mathrm{NPs}$ with a rough surface is significantly higher, i.e. $10.3 \mathrm{wt} \%$, compared to that of spherical NPs, i.e. $3.0 \mathrm{wt} \%$. This nearly 3.4 fold increase in PDMS grafted weight is ascribed to the increased specific surface area for the $\mathrm{SiO}_{2}-\mathrm{R}$ NPs as shown in Figure S5.1. For the NPs with a diameter of $155 \mathrm{~nm}$ the surface area increased from $33 \mathrm{~m}^{2} \mathrm{~g}^{-1}$ to $116 \mathrm{~m}^{2} \mathrm{~g}^{-1}$ for the spherical and $\mathrm{SiO}_{2}-\mathrm{R}$ NPs, respectively (see Figure S5.1). The nearly 3.6 fold increase in surface area is in good agreement with the 3.4 times higher grafted amount of PDMS. For the $\mathrm{SiO}_{2}-\mathrm{R}$ NPs with diameters of $200 \mathrm{~nm}$ and $120 \mathrm{~nm}$ the increase in PDMS grafting $\mathrm{wt} \%$ were in good agreement with the corresponding increase in surface area as well.
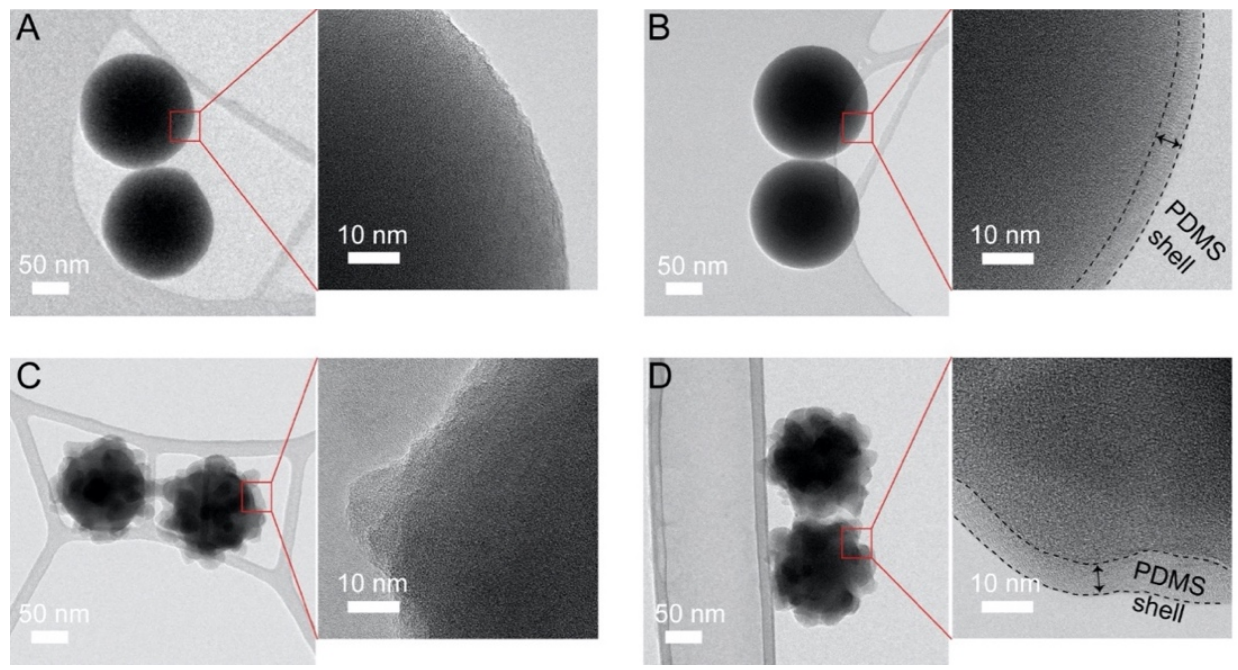

Figure 5.3 TEM images of (a) $155 \mathrm{SiO}_{2}-\mathrm{S}$, (b) $155 \mathrm{SiO}_{2}-\mathrm{SP}$, (c) $155 \mathrm{SiO}_{2}-\mathrm{R}$ and (d) $155 \mathrm{SiO}_{2}-$ RPNPS.

Based on the grafted PDMS mass obtained by TGA measurements, the molar mass of the grafted PDMS chains (i.e. 1,000 $\mathrm{g} \mathrm{mol}^{-1}$ ) and the Brunauer-Emmett-Teller (BET) surface area of the NPs the PDMS grafting densities were calculated to be $\sim 0.6$ chains $\mathrm{nm}^{-2}$ for the $155 \mathrm{SiO}_{2}-\mathrm{SP}$ and $155-\mathrm{SiO}_{2}-\mathrm{RP}$ NPs. This number is lower compared to the earlier reported value of 0.9 for $\mathrm{SiO}_{2}-\mathrm{SP}$ NPs with diameters of $12 \mathrm{~nm}$ to $120 \mathrm{~nm}$, respectively. ${ }^{56}$ This difference is ascribed to the known overestimation of the surface area of $\mathrm{SiO}_{2}$ NPs determined by BET measurements ${ }^{57-59}$ compared to the simple arithmetic estimation of the nanoparticle surface area used, based on the particle diameters. When 
using this simple arithmetic method,grafting density values close to 0.9 chains $\mathrm{nm}^{-2}$ were obtained. This is in good agreement with values for $\mathrm{SiO}_{2}-\mathrm{SP}$ NPs reported in our previous work. ${ }^{60}$ Based on these results we note that BET analysis is used here only to qualitatively show the significant differences in surface area of the particles before and after roughening their surface.

Finally, transmission electron microscope (TEM) was used to confirm the core-shell structure of the PDMS grafted NPs. Figure 5.3a-d shows TEM images of $155 \mathrm{~nm}$ bare and PDMS grafted spherical and $\mathrm{SiO}_{2}-\mathrm{R}$ NPs. From these TEM images it is clear that there is a thin PDMS shell around the $155 \mathrm{SiO}_{2}-\mathrm{SP}$ (Figure 5.3b) and $155 \mathrm{SiO}_{2}-\mathrm{RP}$ (Figure 5.3d) NPs. The tickness of the PDMS shell on the NPs was estimated to be around $6.0 \pm$ $1.5 \mathrm{~nm}$, which agrees well with previous results reported for $\mathrm{SiO}_{2}-\mathrm{SP}$ NPs with silica core diameters from $12 \mathrm{~nm}$ to $120 \mathrm{~nm} .^{60}$

Following successful synthesis and characterization of the NPs we employed them as nucleating agents in $\mathrm{CO}_{2}$ assited batch foaming of PMMA as presented and discussed in the next sections.

\subsubsection{Nanocomposite Foaming and Multinucleation}

Prior to foaming, $\mathrm{SiO}_{2}-\mathrm{R}$ and $\mathrm{SiO}_{2}-\mathrm{RP}$ NPs were melt blended in a PMMA matrix and pressed to films with thickness values of around $200 \mu \mathrm{m} . \mathrm{SiO}_{2}-\mathrm{S}$ and $\mathrm{SiO}_{2}-\mathrm{SP}$ NPs were incorporated in PMMA matrix via the same procedure as control group. For comparison of the nucleation efficiency, NPs with the same volume number density (i.e. $1.07 \times 10^{13}$ particles $\mathrm{cm}^{-3}$ ) were used for each nanocomposite film were used. Thus assuming a good particle dispersion in the PMMA films and that each particle can nucleate the same amount of foam cells, all nanocomposite films had the same volume number density of potential nucleation sites prior to foaming. The particles were well dispersed in PMMA prior to foaming as was observed from SEM images of cross sectioned PMMA nanocomposite films (See Figure S5.3).

The $\mathrm{CO}_{2}$ saturation pressure used for foaming was 55 bar and following quick depressurization (i.e. within 2 seconds), foams were prepared at a foaming temperature of $40{ }^{\circ} \mathrm{C}$ for 1 second. This relatively short foaming time was chosen since we were primarily interested in the nucleation efficiency of the prepared particles and not in the well developed foam morphology at the end stages of foaming (i.e. after several tens of seconds to minutes). Figure 5.4a-d show SEM images of cross sectioned PMMA foams nucleated by $155 \mathrm{SiO}_{2}-\mathrm{S}, 155 \mathrm{SiO}_{2}-\mathrm{SP}, 155 \mathrm{SiO}_{2}-\mathrm{R}$ and $155 \mathrm{SiO}_{2}-\mathrm{RP} \mathrm{NPs}$. It is clear that for the 1 second foaming time the PMMA foams have thick cell walls and struts. 
Furthermore, the $155 \mathrm{SiO}_{2}-\mathrm{S}, 155 \mathrm{SiO}_{2}-\mathrm{SP}$ and $155 \mathrm{SiO}_{2}-\mathrm{R}$ NPs are present at the polymer foam cell wall surface. The $155 \mathrm{SiO}_{2}-\mathrm{S}$ NPs are more embedded in the PMMA cell wall compared to the $155 \mathrm{SiO}_{2}-\mathrm{SP}$, which can be attributed to the low surface energy and high $\mathrm{CO}_{2}$-philicity of the decorated PDMS shell compared with the bare particles, resulting in variations in the strength of the interfacial interactions between the NPs and the polymer gas mixture. ${ }^{60}$ This is in good agreement with the reported results for the nucleation behavior of pristine $\mathrm{SiO}_{2}$ and corresponding PDMS grafted core shell NPs with (core) diameters up to $120 \mathrm{~nm}$. $^{28,60}$

Interestigly, when examining the $155 \mathrm{SiO}_{2}-\mathrm{RP}$ NPs embedded in the PMMA foams we observed that most of these particles are surrounded by a few primary polymer foam cells (see Figure 5.4d). Of particular interest is that the cell walls in between these primary foam cells are much thinner compared to the non nucleated/expanded PMMA matrix surrounding them and that the size of the cells around the $155 \mathrm{SiO}_{2}-\mathrm{RP}$ particles is fairly uniform. This points towards simultaneous multiple heterogeneous nucleation events per added particle. In addition, the thin wall between some foam cells appears to be ruptured, or partly disappeared. This indicates that cell coalescence occurs during the initial stages of nucleation. An explanation for this behavior is provided later.

The presence of multiple nucleation events per $155 \mathrm{SiO}_{2}-\mathrm{RP}$ particle is promising for developing nanocellular foams with increased cell densities. To exploit this option, it is of pivotal importance to obtain a deeper understanding of cell nucleation as a function of the surface roughening feature sizes. Therefore, we have prepared $\mathrm{SiO}_{2}-\mathrm{R}$ and $\mathrm{SiO}_{2}-\mathrm{RP}$ NPs with a diameter of $\sim 80 \mathrm{~nm}, \sim 120 \mathrm{~nm}$ and $\sim 200 \mathrm{~nm}$ to explore this idea. Figure S5.2 shows SEM images of $80 \mathrm{SiO}_{2}-\mathrm{R}, 120 \mathrm{SiO}_{2}-\mathrm{R}$ and $200 \mathrm{SiO}_{2}-\mathrm{R}$ NPs. It is clear that the feature sizes of the roughened surfaces are different for the particles with different diameters, i.e. $80 \mathrm{SiO}_{2}-\mathrm{R}$ has only a few small protrusions at its surface, while $200 \mathrm{SiO}_{2}$ $\mathrm{R}$ reveals numerous larger protruding features (Figure S5.2). It turned out that for the etching method used the initial $\mathrm{SiO}_{2} \mathrm{NP}$ size has an impact on the particle roughness. ${ }^{52}$

The SEM images of the cross sectioned PMMA foams nucleated by $80 \mathrm{SiO}_{2}-\mathrm{RP}$ (Figure 5.4e) and 200 $\mathrm{SiO}_{2}-\mathrm{RP}$ (Figure 5.4f) NPs reveal distinct differences in the foam morphology, as well. For instance, $200 \mathrm{SiO}_{2}-\mathrm{RP}$ clearly shows the presence of multiple cells around single nucleating particles, however, no multiple cell nucleation events are observed around single $80 \mathrm{SiO}_{2}$-RP NPs. 

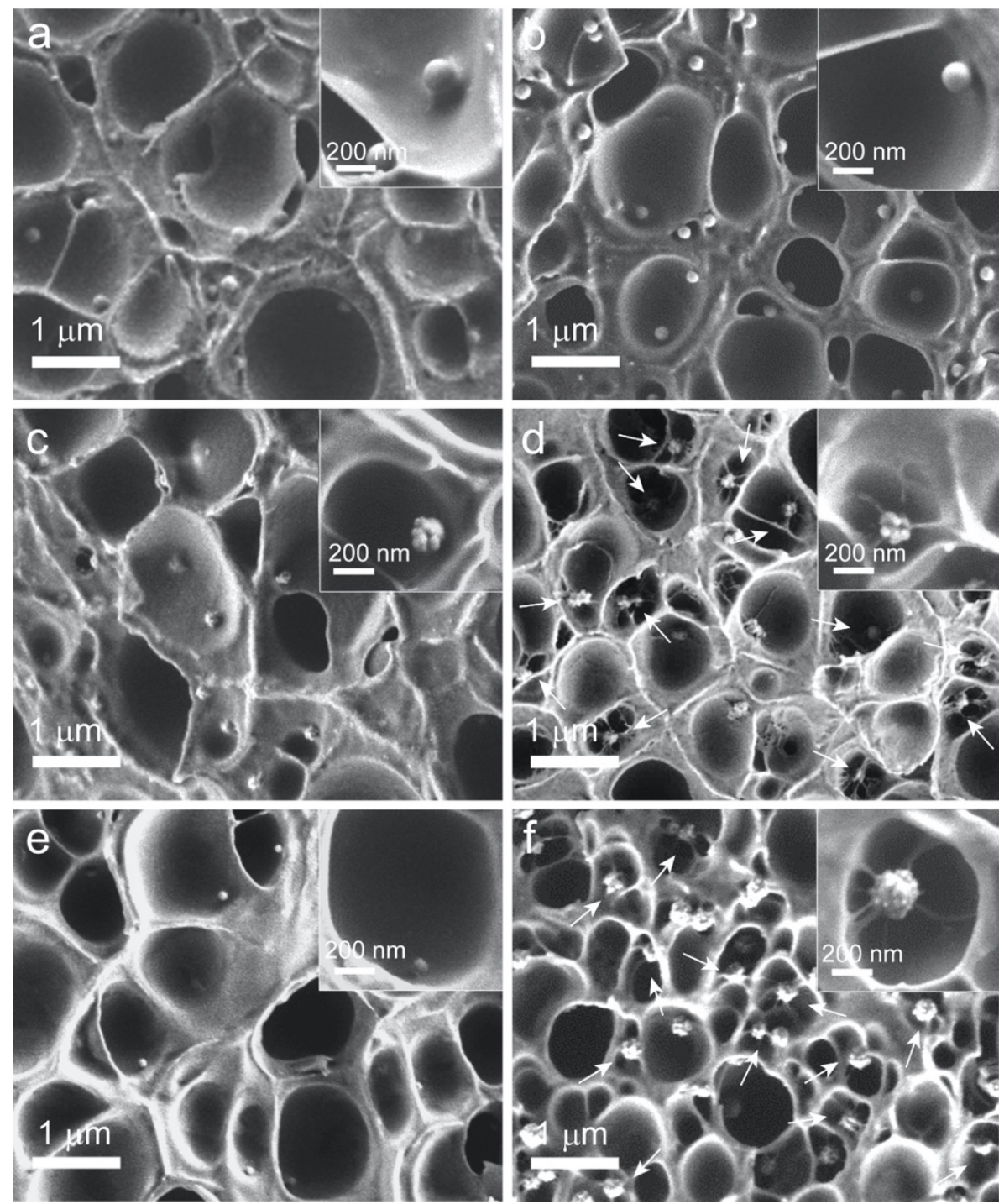

Figure 5.4 SEM images of cross sectioned PMMA foams nucleated by (a) 155 $\mathrm{SiO}_{2}-\mathrm{S}$, (b) $155 \mathrm{SiO}_{2}-\mathrm{SP}$, (c) $155 \mathrm{SiO}_{2}-\mathrm{R}$, (d) $155 \mathrm{SiO}_{2}-\mathrm{RP}$, (e) $80 \mathrm{SiO}_{2}-\mathrm{RP}$ and (f) $200 \mathrm{SiO}_{2}-\mathrm{RP} \mathrm{NPs}$. The white arrows in $(d)$ and $(f)$ point towards the particles showing multiple cell nucleation events per particle. The insets show higher magnification images of nucleating NPs in cross sectioned PMMA foam cells. The saturation pressure, foaming temperature and foaming time were 55 bar, $40^{\circ} \mathrm{C}$ and 1 second, respectively.

Below we present quantitatively how the nucleation efficiency of $\mathrm{SiO}_{2}-\mathrm{RP}$ NPs depends on the particles surface roughness for a PMMA foamed in 1 second. Figure 5.5 shows the cell size (4a), cell density (4b) and nucleation efficiency (4c) values for foams 
nucleated by $\mathrm{SiO}_{2}-\mathrm{S}, \mathrm{SiO}_{2}-\mathrm{SP}, \mathrm{SiO}_{2}-\mathrm{R}$, and $\mathrm{SiO}_{2}-\mathrm{RP}$ NPs as a function of the silica particle diameter.
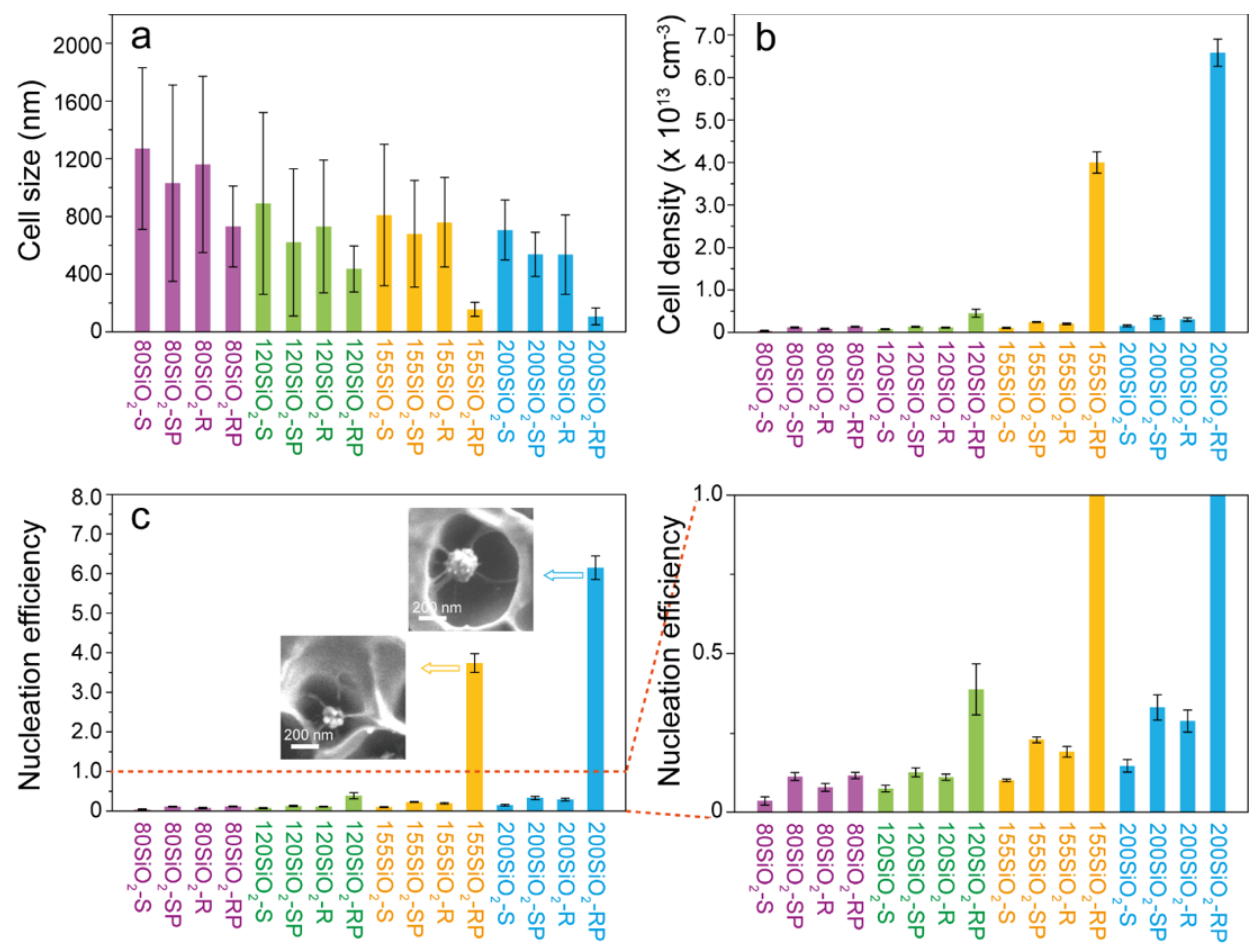

Figure 5.5 (a) Foam cell size, (b) cell density and (c) cell nucleation efficiency for PMMA foams nucleated by $\mathrm{SiO}_{2}-\mathrm{S}, \mathrm{SiO}_{2}-\mathrm{SP}, \mathrm{SiO}_{2}-\mathrm{R}$ and $\mathrm{SiO}_{2}-\mathrm{RP}$ with diameters of $\sim 80 \mathrm{~nm}$, $\sim 120 \mathrm{~nm}, \sim 155 \mathrm{~nm}$ and $\sim 200 \mathrm{~nm}$, respectively. The saturation pressure, foaming temperature and foaming time were $55 \mathrm{bar}, 40^{\circ} \mathrm{C}$ and 1 second, respectively. The inserts in Figure 5.5c show SEM images of cross sectioned PMMA foam cells showing the presence of multiple cells around single $155 \mathrm{SiO}_{2}-\mathrm{RP}$ and $200 \mathrm{SiO}_{2}-\mathrm{RP} \mathrm{NPS}$.

As can be clearly seen from Figure 5.5a, the average cell size nucleated with $\mathrm{SiO}_{2}-\mathrm{R}$ and $\mathrm{SiO}_{2}-\mathrm{RP}$ NPs are smaller compared to $\mathrm{SiO}_{2}-\mathrm{S}$ and $\mathrm{SiO}_{2}-\mathrm{SP}$ NPs with the same diameter, respectively. In addition, upon increasing the particale diameter from 80 to 200 $\mathrm{nm}$, the average cell size decreases and the cell size distribution becomes narrower. SEM images of cross sectioned PMMA foams nucleated by $80 \mathrm{SiO}_{2}-\mathrm{RP}$ particles did not reveal any multiple cell nucleation events per particle (see Figure 5.4e), while for $120 \mathrm{SiO}_{2}-\mathrm{RP}$ this was observed for some particles (Figure S5.4). The decrease of multiple nucleation events is ascribed to a lower surface roughness of the $80-\mathrm{SiO}_{2}-\mathrm{RP}$ and $120 \mathrm{SiO}_{2}-\mathrm{RP} \mathrm{NPs}$, resulting in a reduced number of effective nanocativities. However, for PMMA foams nucleated by $155 \mathrm{SiO}_{2}-\mathrm{RP}$ and $200 \mathrm{SiO}_{2}-\mathrm{RP}$ particles multiple cell nucleation events were 
observed, which resulted in a higher $\mathrm{CO}_{2}$ consumption during cell growth compared to the foams without multiple cell nucleation events occuring. This explains the reduction in cell size to $160 \pm 50 \mathrm{~nm}$ and $110 \pm 60 \mathrm{~nm}$ for foams with $155 \mathrm{SiO}_{2}-\mathrm{RP}$ and $200 \mathrm{SiO}_{2}-$ RP particles, respectivley.

The occurance of multiple cell nucleation events for $\mathrm{SiO}_{2}-\mathrm{RP}$ NPs with various roughnesses has, as expected, a significant effect on the cell density and nucleation effciency values (Figure 5.5b and c). The use of $200 \mathrm{SiO}_{2}-\mathrm{RP}$ NPs results in, similar to the $155 \mathrm{SiO}_{2}-\mathrm{RP}$ NPs, a significant boost in cell density and cell nucleation efficiency compared to the other particle types. In fact, for the $200 \mathrm{SiO}_{2}-\mathrm{RP}$ NPs the nucleation efficiency was $\sim 6.2$, which is the highest nucleation efficiency reported so far.

In addition, $120 \mathrm{SiO}_{2}-\mathrm{R}$ and $120 \mathrm{SiO}_{2}-\mathrm{RP}$ particles have a higher nucleation efficiency compared to $120 \mathrm{SiO}_{2}-\mathrm{S}$ and $120 \mathrm{SiO}_{2}-\mathrm{SP}$, respectively, as well (see Figure $5.5 \mathrm{c}$ ). This is again qualitatively explained by the presence of the nanocavities. However, $80 \mathrm{SiO}_{2}-\mathrm{RP}$ show a comparable nucleation efficiency with $80 \mathrm{SiO}_{2}-\mathrm{SP}$, which is ascribed to presence of mainly convex surface domains at its particle surface. Compared to concave surface roughness, convex structures have a higher cell nulceation free energy. ${ }^{41,42}$

From Figure 5.5 it is obvious that for the particle sizes evaluated the $\mathrm{SiO}_{2}-\mathrm{R}$ NPs have nucleation efficiencies comparable to $\mathrm{SiO}_{2}-\mathrm{SP}$ NPs, while the $\mathrm{SiO}_{2}-\mathrm{S}$ particles are the least effective nucleating agents. Overall it is shown that NPs designed with a concave surface roughness are more efficient when used as nucleation agents compared to the nearly spherical ones and that a combination of roughness with a $\mathrm{CO}_{2}$-philic low surface energy PDMS shell has a very large synergetic effect on PMMA foam cell nucleation.

\subsubsection{Multiple Foam Cells Nucleation Mechanism}

The synergetic effect of a PDMS shell with the presence of cavities on the nucleation efficiency of $\mathrm{SiO}_{2}$-RP NPs was much higher than expected. In fact, we reason that for the higher number of cells to grow, more $\mathrm{CO}_{2}$ as a blowing agent must be available. Thus the $\mathrm{CO}_{2}$ absorption of PMMA films containing $\mathrm{SiO}_{2}-\mathrm{S}, \mathrm{SiO}_{2}-\mathrm{SP}, \mathrm{SiO}_{2}-\mathrm{R}$ and $\mathrm{SiO}_{2}-\mathrm{RP}$ particles with diameters of $\sim 80 \mathrm{~nm}, \sim 120 \mathrm{~nm}, \sim 155 \mathrm{~nm}$ and $\sim 200 \mathrm{~nm}$ were determined in order to further elucidate the cell nucleation efficiency as a function of the particle type and size. The results are shown in Figure 5.6a. As is obvious, PMMA films containing $\mathrm{SiO}_{2}-\mathrm{S}$ and $\mathrm{SiO}_{2}-\mathrm{SP}$ have no significant differences in $\mathrm{CO}_{2}$ absorption regardless of the particle diameter as compared to PMMA without NPs. The same is observed for $80 \mathrm{SiO}_{2}-$ $\mathrm{R}$ and $80 \mathrm{SiO}_{2}-\mathrm{RP}$ NPs. Hence for these particles the overall $\mathrm{CO}_{2}$ concentration is not altered while locally in the PMMA films the $\mathrm{CO}_{2}$ concentration may vary due to the 
presence of the PDMS shell. Upon increasing the surface roughness of the particles, e.g. for the $120 \mathrm{SiO}_{2}-\mathrm{R}, 155 \mathrm{SiO}_{2}-\mathrm{R}$ and $200 \mathrm{SiO}_{2}-\mathrm{R} \mathrm{NPs}$, the amount of $\mathrm{CO}_{2}$ absorbed is expected to significantly increase. The presence of a PDMS shell increases the amount absorbed even more for these NPs. For instance, for $200 \mathrm{SiO}_{2}-\mathrm{R}$ and $200 \mathrm{SiO}_{2}-\mathrm{RP}$ the increase in $\mathrm{CO}_{2}$ absorbed was $\sim 4.7 \mathrm{wt} \%$ and $\sim 8.8 \mathrm{wt} \%$, respectivley, compared to the PMMA containing no particles $(\sim 18.2 \mathrm{wt} \%)$.

This increase in $\mathrm{CO}_{2}$ absorption for the rough particles is ascribed to capilary condensation of $\mathrm{CO}_{2}$ in the nanocavities. ${ }^{62-64}$ The even larger increase in $\mathrm{CO}_{2}$ absorption for the rough particles with a PDMS shell with diamers of $\sim 120 \mathrm{~nm}, \sim 155 \mathrm{~nm}$ and $\sim 200$ $\mathrm{nm}$ compared to their bare counterparts is ascribed to the presence of $\mathrm{CO}_{2}$-philic molecules attached to the nanocavity surfaces, which is known to potentially enhance the absorption of $\mathrm{CO}_{2}{ }^{65-67}$

The higher physical blowing agent concentration close to the particle surface is favorable for increasing the nucleation rate and combined with the presence of a low surface energy PDMS shell in the nanocavities the foam cell nucleation efficiency of these particles is significantly enhanced. Figure $5.6 \mathrm{~b}$ shows a schematic representation of the proposed mechanism leading to multiple cell nucleation events for PDMS grafted $\mathrm{SiO}_{2}-\mathrm{R}$. Upon saturation of PMMA films containing $\mathrm{SiO}_{2}-\mathrm{RP} \mathrm{NPs}, \mathrm{CO}_{2}$ capillary condensation in the cavities results in the presence of significantly increased amounts of $\mathrm{CO}_{2}$ in close proximity of the nucleating particle surface. The nanocavities are energetically favorable nucleating sites and together with the increased amount of available $\mathrm{CO}_{2}$, result in more than 1 nucleating event per particle (step A), subsequent cell growth (step B), and cell coalescence (step C). This is further experimentally confirmed by the development of the foam cell morphology as a function of the foaming time for PMMA foams nucleated by $155 \mathrm{SiO}_{2}-\mathrm{RP}$ NPs, as shown in Figure 5.6c-f. Upon increasing of foaming time, the cell walls in between these primary foam cells nucleated from the same single $155 \mathrm{SiO}_{2}-\mathrm{RP}$ appears to be ruptured or partly disappeared, leading to the cells coalescence. In addtion, the cell size and cell nucleation efficiency for PMMA foams, obtained with $155 \mathrm{SiO}_{2}-\mathrm{S}, 155 \mathrm{SiO}_{2}-\mathrm{SP}, 155 \mathrm{SiO}_{2}-\mathrm{R}$ and $155 \mathrm{SiO}_{2}-\mathrm{RP} \mathrm{NPs}$, were also determined as a function of the foaming time (see Figure S5.5). 


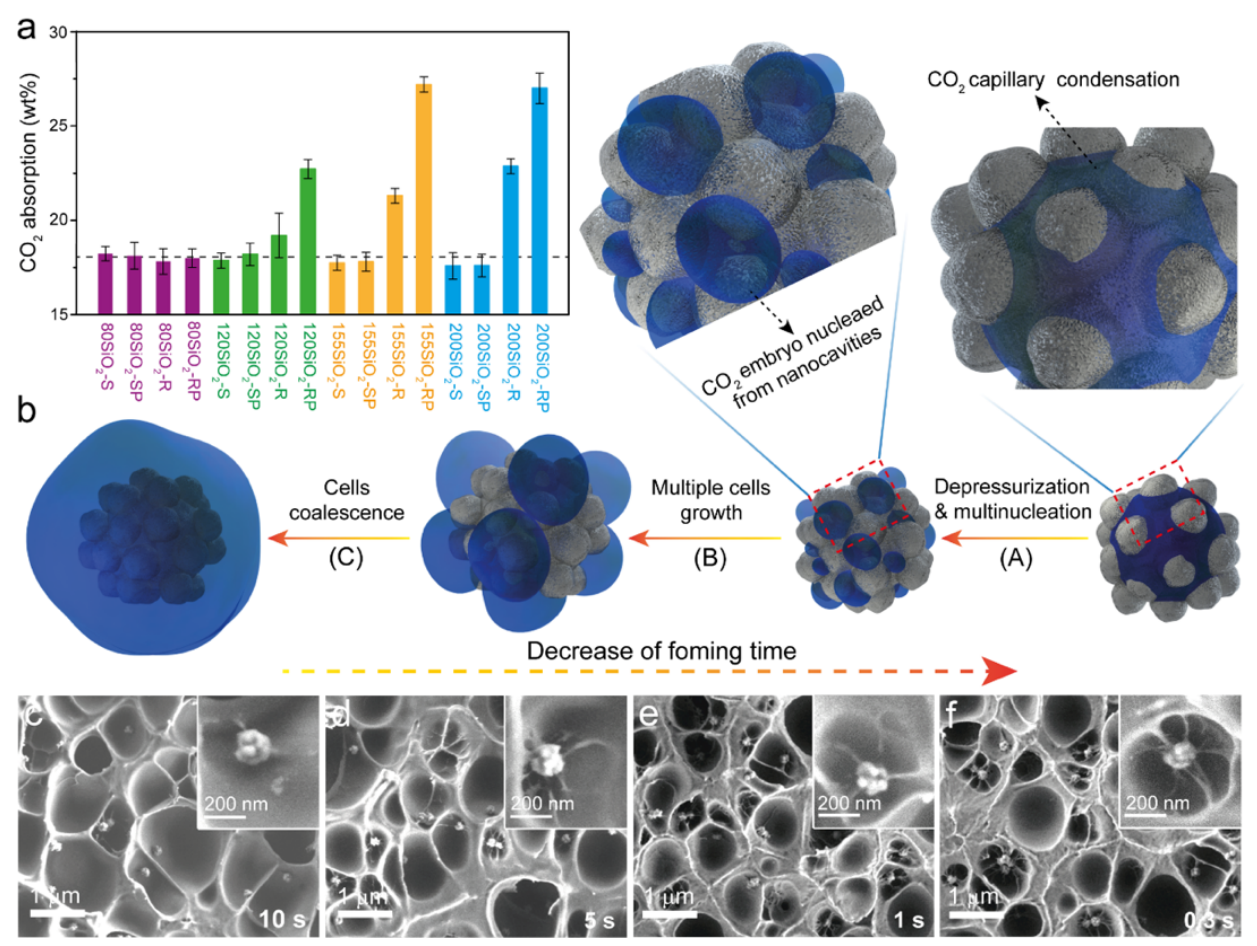

Figure 5.6 (a) $\mathrm{CO}_{2}$ absorption for PMMA films containing $\mathrm{SiO}_{2}-\mathrm{S}, \mathrm{SiO}_{2}-\mathrm{SP}, \mathrm{SiO}_{2}-\mathrm{R}$ and $\mathrm{SiO}_{2}-\mathrm{RP}$ particles with diameters between $80 \mathrm{~nm}$ to $200 \mathrm{~nm}$. The saturation pressure and time were 55 bar and 4 h, respectively. The dashed line shows the $\mathrm{CO}_{2}$ absorption of PMMA containing no NPs. (b) Schematic representation of the nucleation of multiple cells from PDMS grafted $\mathrm{SiO}_{2}-\mathrm{R}$ NPS. SEM images of cross sectioned PMMA foams nucleated by $155 \mathrm{SiO}_{2}-\mathrm{RP} \mathrm{NPs}$ for (c) 10 seconds, (d) 5 second, (e) 1 seconds and (f) 0.3 seconds. In the insets, the foam cell wall around a single nanoparticle is shown. The saturation pressure and foaming temperature were 55 bar and $40^{\circ} \mathrm{C}$, respectively.

Overall, we presented that by incorporating PDMS decorated $\mathrm{SiO}_{2}-\mathrm{R}$ NPs in PMMA matrix, multiple cell nucleation events per nanoparticle can be achieved. The designer NPs we developed and prepared in this Thesis exhibit significantly higher cell nucleation efficiency in PMMA nanocellular foaming compared to their bare and nearly spherical counterparts. We have also exploited $155 \mathrm{SiO}_{2}-\mathrm{RP}$ NPs in the $\mathrm{CO}_{2}$ assisted batch foaming of polystyrene and the corresponding SEM images revealed the occurrence of multiple cell nucleation events as well (see Figure S5.6). Hence, these hybrid core shell NPs are very promising as highly efficient cell nucleation agents in polymer nanocellular foaming in general.

To fully exploit the high nucleation efficiency of $\mathrm{SiO}_{2}-\mathrm{RP}$ particles it is of pivotal importance to find foaming strategies and foam matrices that allow both the introduction 
of a high number of cell nuclei and also ensure early cell stabilization during the foaming process to prevent cell coalescence. For instance, without optimization of any of the foaming parameters and or composition of the PMMA nanocomposite matrix we have obtained a high cell density of $\sim 6.6 \times 10^{13}$ cells $\mathrm{cm}^{-3}$, which is already very close to the order of magnitude of interest for superior thermal isolation (i.e. $10^{14}$ cells cm $\mathrm{cm}^{-3}$ ). Future work must be directed to developing new strategies for sufficiently increasing the surface roughness of NPs with a particle diameter $<100 \mathrm{~nm}$. This is essential since at the same weight percentage of particle loading a higher number of effective nucleation agents is available when smaller particles are used.

\subsection{Conclusion}

In summary, $\mathrm{SiO}_{2}-\mathrm{R}$ NPs were synthesized via attaching and etching approaches. Particles synthesized via the attaching method showed unevenly distributed smaller particles on the larger core particles. The fact that the attaching method can only achieve partial coverage and the long reaction time required, directed us towards exploring the etching method to prepare $\mathrm{SiO}_{2}-\mathrm{R}$ NPs. The etching method resulted in the uniform formation of small (20-30 nm) silica domains covering the surface of $\mathrm{SiO}_{2}-\mathrm{R} \mathrm{NPs}$. The $\mathrm{SiO}_{2}-\mathrm{R}$ NPs synthesized via the etching method were successfully exploited as efficient nucleation agents in $\mathrm{CO}_{2}$-blown PMMA nanocellular foaming. Compared to nearly spherical $\mathrm{SiO}_{2} \mathrm{NPs}, \mathrm{SiO}_{2}-\mathrm{R}$ NPs exhibit a higher nucleation efficiency after being incorporated in the PMMA matrix due to the lower critical nucleation energy. Multiple nucleating events per particle were observed for $\mathrm{SiO}_{2}$-RP NPs with core diameters of 155 $\mathrm{nm}$ and $200 \mathrm{~nm}$, respectively. The highest nucleation efficiency obtained was 6.2 for $200 \mathrm{SiO}_{2}$-RP NPs. The significantly higher nucleation efficiency (well above unity) of the core-shell surface roughed silica NPs is attributed to the synergetic effect of the thin PDMS shell combined with the presence of nanocavities on the NPs' surface as well as the higher $\mathrm{CO}_{2}$ absorption. This results in an increased physical blowing agent concentration available for nucleation close to the energetically favorable nanocavities as nucleating sites. Hence, PDMS decorated, core-shell surface roughed silica NPs are very promising to be used as an interesting class of highly efficient nucleation agents. To increase our understanding of multiple cell nucleation events by PDMS decorated coreshell surface roughed silica NPs, future work should be directed to the quantification of the relationship between nucleation energy barrier and surface cavity structure dimensions, as well optimization of foaming conditions to stabilize the nucleated multiple cells during the early stages of foaming. 


\subsection{Materials and Methods}

Materials. PMMA was obtained from Arkema (VM100, i.e. a PMMA-co-EA polymer, $\rho=1.18 \mathrm{~g} \mathrm{~cm}^{-3}$ ) (La Garenne-Colombes, France). Absolute ethanol for analysis was purchased from Merck (Darmstadt, Germany). Absolute tetrahydrofuran (THF), 2propanol 99.8\% and chloroform 99.9\% were acquired from Biosolve (Valkenswaard, the Netherlands). Monoglycidyl ether terminated poly(dimethylsiloxane) (PDMS-G) (Mw = $1000 \mathrm{~g} \mathrm{~mol}^{-1}$ ), ammonium hydroxide solution 28 -30\%, (3-aminopropyl)triethoxysilane $\geq 98 \%$ (APTES), Sodium borohydride purum p.a., $\geq 96 \%$ (gas-volumetric) $\left(\mathrm{NaBH}_{4}\right)$, polyvinylpyrrolidone with a molar mass of 40,000 $\mathrm{g} \mathrm{mol}^{-1}$ (PVP40), (3Glycidyloxypropyl)trimethoxysilane $\geq 98 \%$ (GPS), and tetraethyl orthosilicate (TEOS) $\geq$ 99.0\% were purchased from Sigma-Aldrich (St. Louis, MO, USA). Milli-Q water was produced by a Millipore Synergy System (Billerica, MA, USA). Unless otherwise mentioned, all other chemicals were used as received.

Stöber Silica Nanoparticle Synthesis. Nearly smooth Stöber silica nanoparticles $\left(\mathrm{SiO}_{2}-\mathrm{S}\right.$ NPs$)$ with a diameter of $\sim 80 \mathrm{~nm}, \sim 120 \mathrm{~nm}, \sim 155 \mathrm{~nm}, \sim 180 \mathrm{~nm} \sim 200 \mathrm{~nm}, \sim$ $310 \mathrm{~nm}$ and $600 \mathrm{~nm}$ were prepared by the Stöber method. To prepare $\mathrm{SiO}_{2}-\mathrm{S}$ NPs with a diameter of $\sim 155 \mathrm{~nm}, 100 \mathrm{ml}$ ethanol was mixed with $8 \mathrm{ml}$ Milli-Q water and $10 \mathrm{ml}$ TEOS in the presence of $5 \mathrm{ml}$ ammonium hydroxide while stirring at $500 \mathrm{rpm}$ at $50{ }^{\circ} \mathrm{C}$. After 4.5 hours the $\mathrm{SiO}_{2}-\mathrm{S}$ NP dispersion obtained was centrifuged at 10,000 rpm for 30 min. Subsequently, the collected $\mathrm{SiO}_{2}-\mathrm{S}$ NPs were redispersed in ethanol and centrifuged again. This washing step was repeated 2 more times followed by vacuum drying the collected $\mathrm{SiO}_{2}-\mathrm{S}$ NPs at room temperature for 12 hours. To prepare $\mathrm{SiO}_{2}-\mathrm{S}$ NPs with a diameter of $\sim 80 \mathrm{~nm}, 168 \mathrm{ml}$ ethanol was mixed with $28 \mathrm{ml}$ Milli-Q water and $30 \mathrm{ml}$ TEOS in the presence of $2 \mathrm{ml}$ ammonium hydroxide while stirring for 1.5 hours at 500 $\mathrm{rpm}$ at room temperature. To synthesize the $\sim 120 \mathrm{~nm}$ particles $100 \mathrm{ml}$ ethanol was mixed with $8 \mathrm{ml}$ Milli-Q water and $5 \mathrm{ml}$ TEOS in a round bottom flask stirring at $500 \mathrm{rpm}$ and subsequently $5 \mathrm{ml}$ of ammonium hydroxide was added and reacted for 3 hours at $50{ }^{\circ} \mathrm{C}$. To synthesize the $\sim 180 \mathrm{~nm}$ particles $100 \mathrm{ml}$ of ethanol was mixed with $8 \mathrm{ml}$ Milli-Q water and $10 \mathrm{ml}$ TEOS in the presence of $5.3 \mathrm{ml}$ ammonium hydroxide in a $250 \mathrm{ml}$ round bottom flask while stirring at $500 \mathrm{rpm}$. The reaction was conducted for 4.5 hours at 50 ${ }^{\circ} \mathrm{C}$. To synthesize the $\sim 200 \mathrm{~nm}$ particles $100 \mathrm{ml}$ of ethanol was mixed with $8 \mathrm{ml}$ Milli-Q water and $10 \mathrm{ml}$ TEOS in the presence of $5.6 \mathrm{ml}$ ammonium hydroxide in a $250 \mathrm{ml}$ round bottom flask while stirring at $500 \mathrm{rpm}$. The reaction was conducted for 4.5 hours at 50 ${ }^{\circ} \mathrm{C}$. To synthesize the $\sim 310 \mathrm{~nm}$ particles $100 \mathrm{ml}$ ethanol was mixed with $8 \mathrm{ml}$ Milli-Q water and $10 \mathrm{ml}$ TEOS in a round bottom flask stirring at $500 \mathrm{rpm}$ and subsequently 7.0 
$\mathrm{ml}$ of ammonium hydroxide was added and reacted for 4.5 hours at $50{ }^{\circ} \mathrm{C}$. To synthesize the $\sim 600 \mathrm{~nm}$ particles $75 \mathrm{ml}$ 2-propanol was mixed with $25 \mathrm{ml}$ methanol and $10 \mathrm{ml}$ TEOS in a round bottom flask stirring at $270 \mathrm{rpm}$ and subsequently $21 \mathrm{ml}$ of ammonium hydroxide was added and reacted for $24 \mathrm{~h}$ at room temperature. The collecting, washing and drying steps of NPs were the same as described for NPs of $\sim 155 \mathrm{~nm}$.

Silica Nanoparticle Hydrolysis. To introduce silanol groups on the surface of the prepared $\mathrm{SiO}_{2}-\mathrm{S}$ NPs, these were redispersed in Milli-Q water by sonication (BRANSON 2510, Canada) for 1 hour. Subsequently, hydrochloric acid was added to the dispersion while stirring at $500 \mathrm{rpm}$ until the $\mathrm{pH}$ of the solution reached a value of approximately 1 . After 4 hours the dispersion was centrifuged at 10,000 rpm for $30 \mathrm{~min}$. The collected NPs were redispersed in Milli-Q water and centrifuged again. This washing step was repeated 2 more times followed by drying the silanol functional NPs $\left(\mathrm{SiO}_{2}-\mathrm{OH}\right)$ under vacuum at room temperature for 12 hours.

Amino-Functionalization of Silica Nanoparticles. $1.5 \mathrm{~g} \mathrm{SiO}_{2}-\mathrm{OH}$ NPs were redispersed in $50 \mathrm{ml}$ ethanol followed by the addition of $7.5 \mathrm{ml}$ APTES. The dispersion was left to stir at $500 \mathrm{rpm}$ at room temperature for 17 hours. The APTES functionalized NPs $\left(\mathrm{SiO}_{2}-\mathrm{NH}_{2}\right)$ were collected by centrifugation at $10,000 \mathrm{rpm}$ for $30 \mathrm{~min}$ and redispersed in ethanol and centrifuged again. This washing step was repeated 2 more times followed by drying the collected $\mathrm{SiO}_{2}-\mathrm{NH}_{2} \mathrm{NPs}$ under vacuum at room temperature for 12 hours.

Epoxy-Functionalization of Silica Nanoparticles. $1.5 \mathrm{~g}$ of $\mathrm{SiO}_{2}-\mathrm{S}(600 \mathrm{~nm})$ were re-dispersed in $40 \mathrm{~mL}$ dry toluene, followed by addition of $0.2 \mathrm{~g}$ GPS in $5 \mathrm{~mL}$ dry toluene in dropwise under vigorous stirring. The suspension was stirred at $50{ }^{\circ} \mathrm{C}$ under $\mathrm{N}_{2}$ atmosphere for $24 \mathrm{~h}$. The GPS grafted $\mathrm{SiO}_{2} \mathrm{NPs}\left(\mathrm{SiO}_{2}-\mathrm{GPS}\right)$ were collected by centrifugation and washed with toluene twice, then vacuum dried at $50{ }^{\circ} \mathrm{C}$ for $12 \mathrm{~h}$.

Grafting to of PDMS-G to Silica Nanoparticles. $1.0 \mathrm{~g}$ of $\mathrm{SiO}_{2}-\mathrm{NH}_{2} \mathrm{NPs}$ were redispersed in $20.5 \mathrm{ml}$ THF and $15 \mathrm{~g}$ PDMS-G while stirring at $500 \mathrm{rpm}$ for 1 hour followed by sonication for 1 hour. Subsequently, THF was removed by rotary evaporation and the resulting silica nanoparticle dispersion in PDMS-G was immersed in an oil bath thermostated at $80{ }^{\circ} \mathrm{C}$ for $17 \mathrm{~h}$. Following cooling to room temperature the reaction mixture was washed with $\mathrm{THF}$ and centrifuged at $10,000 \mathrm{rpm}$ for $30 \mathrm{~min}$. This washing step was repeated 2 more times, followed by vacuum drying the PDMS-G grafted silica NPs $\left(\mathrm{SiO}_{2}-\mathrm{SP}\right)$ at room temperature for 12 hours.

\section{Synthesis and Modification of Surface Roughness Engineered Silica}


Nanoparticles via the Attaching Method. $0.3 \mathrm{~g} \mathrm{SiO}_{2}-\mathrm{NH}_{2}(80 \mathrm{~nm})$ were suspended in $20 \mathrm{~mL}$ ethanol. Next, $0.6 \mathrm{~g}$ of $\mathrm{SiO}_{2}$-GPS $(600 \mathrm{~nm})$ were suspended in $15 \mathrm{ml}$ ethanol, which were then added dropwise to the $\mathrm{SiO}_{2}-\mathrm{NH}_{2}$ suspension. The suspension was refluxed at $70{ }^{\circ} \mathrm{C}$ for $24 \mathrm{~h}$ under $\mathrm{N}_{2}$ flux. The $\mathrm{SiO}_{2}-\mathrm{R}$ were collected by centrifugation and washed with ethanol twice, followed by vacuum drying at $50{ }^{\circ} \mathrm{C}$ for $16 \mathrm{~h}$.

Synthesis and Modification of Surface Roughness Engineered Silica Nanoparticles via the Etching Method. To synthesize NPs with enhanced surface roughness of $\sim 80 \mathrm{~nm}, 0.3 \mathrm{~g} \mathrm{SiO}_{2}-\mathrm{S}$ NPs of $\sim 80 \mathrm{~nm}$ and $0.25 \mathrm{~g}$ PVP were redispersed in $10 \mathrm{ml}$ Milli-Q water by sonication for $30 \mathrm{~min}$. Subsequently, $0.6 \mathrm{~g} \mathrm{NaBH} 4$ was added to the dispersion while stirring at $500 \mathrm{rpm}$ at room temperature for another $30 \mathrm{~min}$. The resulting mixture was reacted at $50{ }^{\circ} \mathrm{C}$ for 1 hour and $15 \mathrm{~min}$. Stöber silica NPs with diameters of $\sim 155 \mathrm{~nm}, \sim 180 \mathrm{~nm}$ and $\sim 310 \mathrm{~nm}$ were used to synthesize rough surface NPs of $\sim 120 \mathrm{~nm}, \sim 155 \mathrm{~nm}$ and $\sim 200 \mathrm{~nm}$, respectively, and the reaction were conducted for 3 hours and $20 \mathrm{~min}, 3.5$ hours, and 6 hours, respectively. The other steps for synthesis of these surface rough NPs (with diameters from 120 to $220 \mathrm{~nm}$ ) were the same as described for the synthesis of rough surface NPs of $\sim 80 \mathrm{~nm}$. Subsequently, The obtained NPs featuring rough surfaces were collected by centrifugation at 10,000 rpm for $30 \mathrm{~min}$ and redispersed in Milli-Q water and centrifuged again. This washing step was repeated 2 more times followed by drying the collected NPs in vacuum at room temperature for 12 hours. The process of amino-functionalization and grafting to of PDMS-G to the rough surface NPs were the same as described for the Stöber silica NPs.

Nanocomposite Preparation. Nanocomposites were prepared by dispersing the same amount of (functional) silica NPs $\left(1.07 \times 10^{13} \mathrm{~cm}^{-3}\right)$ in PMMA with a mini extruder (DSM Xplore, the Netherlands). In a typical procedure a dry blend of NPs and PMMA was fed to the extruder followed by internal mixing for 3 minutes. The barrel temperature was set to $155{ }^{\circ} \mathrm{C}$ and the screw speed was $100 \mathrm{rpm}$. Subsequently the PMMA nanocomposite was collected and left to cool to room temperature.

Film Preparation. A hot press (Fontijne, the Netherlands) was used to press $\sim 0.2$ $\mathrm{mm}$ thick nanocomposite films in a mold $(4 \times 3 \mathrm{~cm})$. The press temperature, applied load and press time were $180^{\circ} \mathrm{C}, 250 \mathrm{kN}$ and 10 minutes, respectively.

Batch Foaming of Nanocomposite Films. The nanocomposite PMMA films were saturated with $\mathrm{CO}_{2}(55 \mathrm{bar})$ in an autoclave for 4 hours at room temperature followed by rapid depressurization. Subsequently, the PMMA nanocomposite films were immersed in a water bath thermostated at $40{ }^{\circ} \mathrm{C}$ for different foaming time $(0.3$ second, 1 second, 5 second, 10 second and 30 second) after which the samples were quenched in an ice bath 136 
for 30 minutes. The samples were left to dry in air for at least 12 hours prior to further analysis.

Fourier Transform Infrared Spectroscopy. FTIR spectra were collected with a Bruker ALPHA single attenuated total reflection (ATR) FTIR Spectrometer equipped with an ATR single reflection crystal (Bruker Optic GmbH, Ettlingen, Germany). The spectra were collected in the range of $400-4000 \mathrm{~cm}^{-1}$ (spectral solution of $4 \mathrm{~cm}^{-1}, 128$ scans). Background spectra were recorded against air.

Thermogravimetric Analysis. The weight loss of the (modified) particles as a function of temperature was measured with a TGA400 (PerkinElmer, Inc., Waltham, MA, USA). A sample weighing 3-6 mg was loaded into the platinum pan and the temperature was set to $50{ }^{\circ} \mathrm{C}$ to stabilize. Subsequently the sample was heated to $900{ }^{\circ} \mathrm{C}$ at a heating rate of $20^{\circ} \mathrm{C} \mathrm{min}-1$. The applied air flow was $20 \mathrm{ml} \mathrm{min}$.

Transmission Electron Microscopy. TEM was performed by a FEI/Philips CM300 system (Eindhoven, the Netherlands). Diluted particle dispersions in THF were deposited on the carbon side of a carbon/copper grid (HC200-Cu) (EMS, Germany) prior to imaging. Images were obtained in the bright field mode with a $300 \mathrm{kV}$ acceleration voltage.

Scanning Electron Microscopy. For SEM a high-resolution scanning electron microscope (JEOL Field Emission JSM-633OF, JEOL Benelux, Nieuw-Vennep, the Netherlands) was employed using an acceleration voltage of $5 \mathrm{keV}$. Prior to analysis the nanocomposite foams were freeze fractured following cooling in liquid nitrogen for 5 minutes.

Surface Area Measurements of Nanoparticles. The specific surface area of the NPs was measured by determining nitrogen adsorption isotherm obtained at $300^{\circ} \mathrm{C}$ using an ASAP 2010 Sorptometer (Micromeritics). Around $100 \mathrm{mg}$ nanoparticle powder was used for each measurement.

$\mathrm{CO}_{2}$ Absorption Measurement. To measure the absorption of $\mathrm{CO}_{2}$ PMMA nanocomposite films containing the same amount of the respective NPs were saturated in $\mathrm{CO}_{2}$ at 55 bar for 4 hours. The weight of the PMMA (nanocomposite) films before and after $\mathrm{CO}_{2}$ saturation were measured.

Calculation of PDMS Grafting Density. The grafting density (D) of PDMS decorated NPs surface was determined according to equation 1. 


$$
\mathrm{D}=\frac{K *(m / M)}{S}
$$

eq. 1

$\mathrm{K}=6.02 * 10^{23} \mathrm{~mol}^{-1}$ which is Avogadro's number, $\mathrm{m}$ is the weight of PDMS grafted per gram NPs (determined from TGA). $\mathrm{M}=1,000 \mathrm{~g} \cdot \mathrm{mol}^{-1}$, which is the molar mass of the used PDMS. S is the surface area per gram NPs, which was determined by BET.

Calculation of Cell Density and Nucleation Efficiency. The cell size and cell density were obtained by analyzing the obtained SEM cross sectional images. Cell density $\left(N_{v}\right)$ of the foams was calculated according to Kumar's theoretical approximation. ${ }^{68}$ No direct measurements of cell dimensions over the micrograph are required in this method, only the micrograph area $(A)$ and the total number of cells $(n)$ contained therein are measured. Together with the magnification factor of the micrograph $(M), N_{v}$ can be calculated according to equation 2 .

$$
N_{v}=\left[\frac{\left(n M^{2}\right)}{A}\right]^{3 / 2}
$$

By combining $N_{V}$ with the volume expansion ratio $(B)$ of nanocomposite films after foaming, the cell numbers per $\mathrm{cm}^{3}$ of unfoamed materials $(N)$ can be calculated according to equation 3 .

$$
N=N_{v} * B
$$

eq. 3

The mentioned cell density in the main text are all refer to $N$. In addition, the nucleation efficiency $(f)$ of NPs during foaming can be calculated as:

$$
f=N / C
$$

Where $C$ is the number of NPs per $\mathrm{cm}^{3}$ (i.e., $1.07 \times 10^{13}$ ) used for foaming.

Figure S5.7 shows how we calculated the amount of cells in the occasion of multiple cell nucleating events occurring around a particle. 


\subsection{Supporting Information}

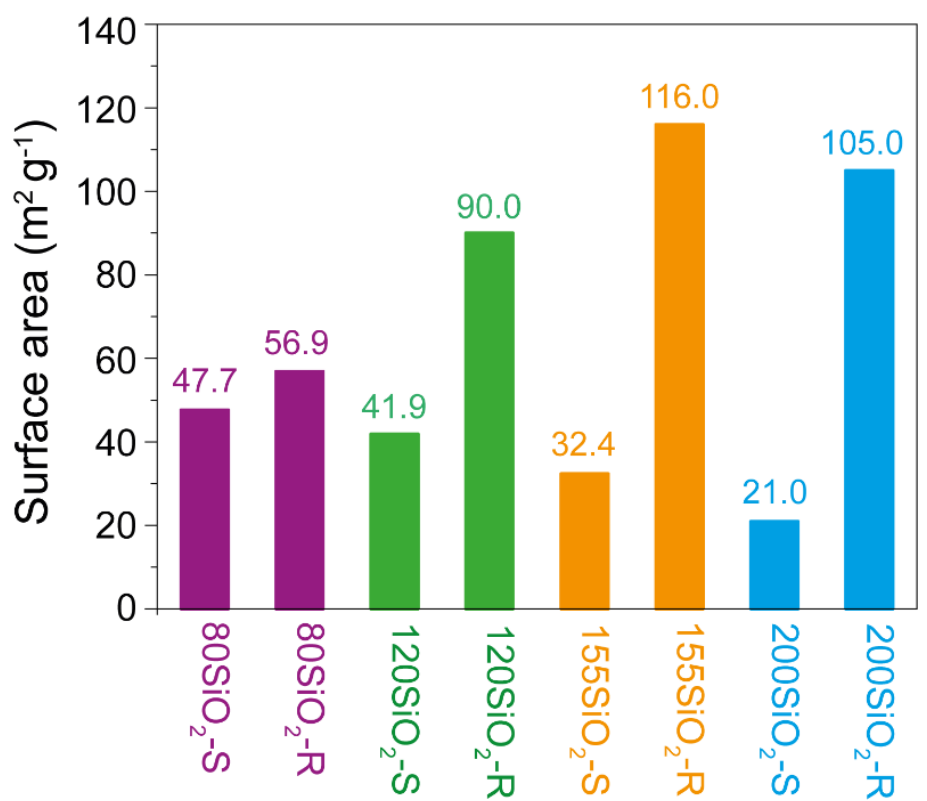

Figure S5.1 The specific surface area determined by BET measurements of $\mathrm{SiO}_{2}-\mathrm{S}$ and $\mathrm{SiO}_{2}-\mathrm{R} N \mathrm{NS}$ with a diameter of $\sim 80 \mathrm{~nm}, \sim 120 \mathrm{~nm}, \sim 155 \mathrm{~nm}$ and $\sim 200 \mathrm{~nm}$.

BET measurements were used to determine the surface area of the prepared silica NPs. The results are shown in Figure S5.1. It is obvious that the surface area of NPs was significantly increased after $\mathrm{NaBH}_{4}$ treatment. For the NPs with a diameter of $155 \mathrm{~nm}$ the surface area increased from $32.4 \mathrm{~m}^{2} \mathrm{~g}^{-1}\left(155 \mathrm{SiO}_{2}-\mathrm{S}\right)$ to $116.0 \mathrm{~m}^{2} \mathrm{~g}^{-1}\left(155 \mathrm{SiO}_{2}-\mathrm{R}\right)$. Nearly 3.6 fold increase in surface area is in good agreement with the 3.4 times higher grafted amount of PDMS to these particles.
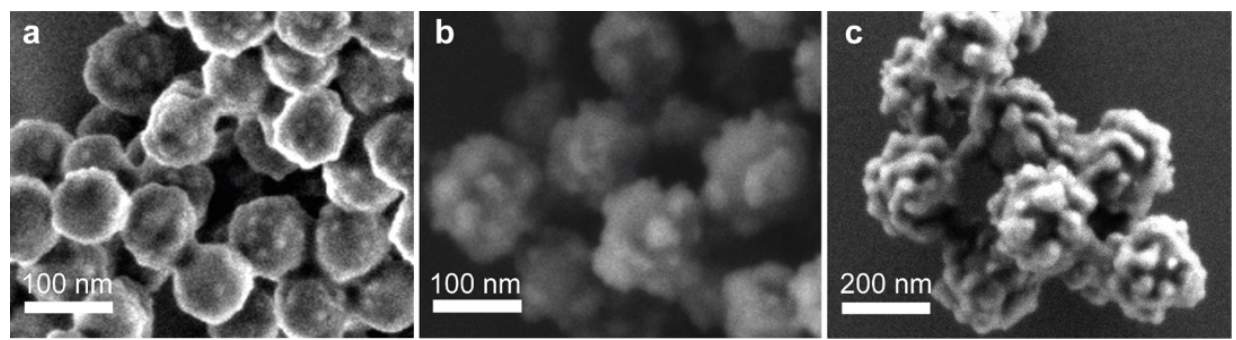

Figure S5.2 SEM images showing $\mathrm{SiO}_{2}-\mathrm{R} N P \mathrm{~s}$ with a diameter of (a) $80 \mathrm{~nm}$, (b) $\sim 120 \mathrm{~nm}$, and (c) $200 \mathrm{~nm}$.

Figure S5.2 shows the SEM images of the $\mathrm{SiO}_{2}-\mathrm{R}$ NPs with diameters of $\sim 80 \mathrm{~nm}, \sim$ 
$120 \mathrm{~nm}$ and $\sim 200 \mathrm{~nm}$. Figure S5.3 shows cross sectional SEM images of the $80 \mathrm{~nm}$ series of particles melt blended in PMMA as a representative case to demonstrate the particle disperion in the composite. From Figure S5.3 it is clear that a good particle dispersion was obtained by melt blending.
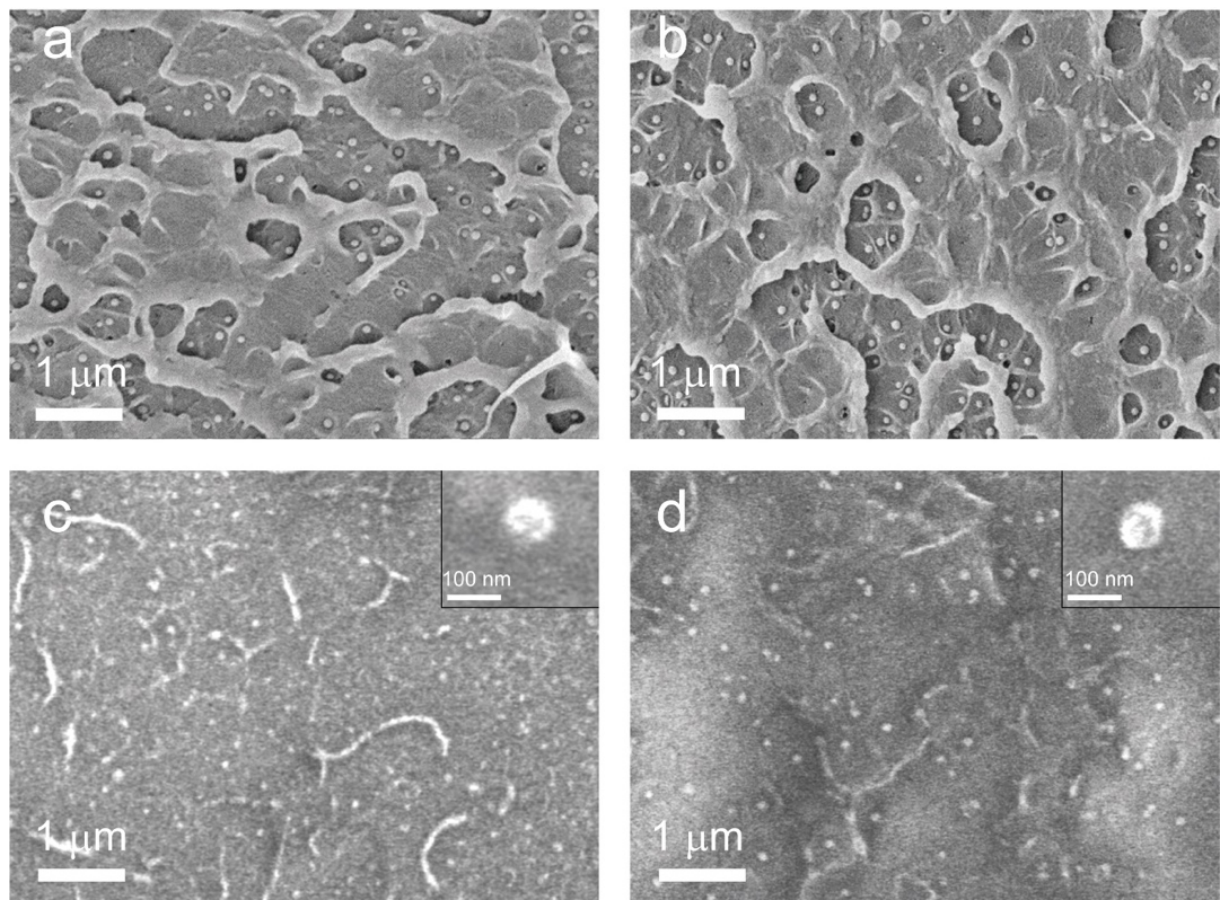

Figure S5.3 Cross sectional SEM images showing the dispersion of (a) $80 \mathrm{SiO}_{2}-\mathrm{S}$, (b) $80 \mathrm{SiO}_{2}-\mathrm{SP}$, (c) $80 \mathrm{SiO}_{2}-\mathrm{R}$ and (d) $80 \mathrm{SiO}_{2}-\mathrm{RP} \mathrm{NPS}$ in PMMA. 


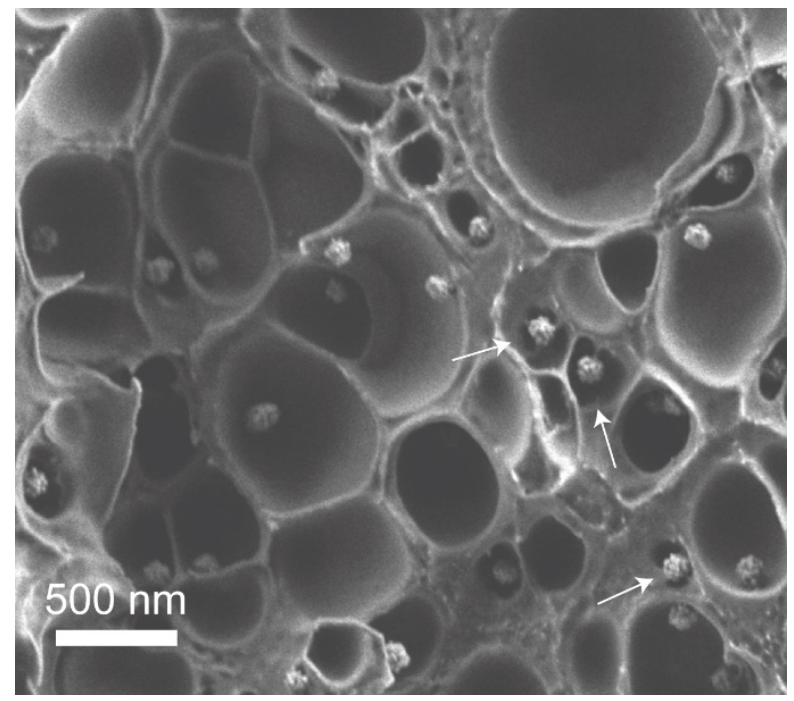

Figure S5.4 SEM images of cross sectioned PMMA foams nucleated by $120 \mathrm{SiO}_{2}-\mathrm{RP} N \mathrm{NS}$. The saturation pressure, foaming temperature and foaming time were $55 \mathrm{bar}, 40{ }^{\circ} \mathrm{C}$ and 1 second, respectively. The white arrows point towards the particles showing multiple cell nucleation events per particle.

Figure S5.4 shows the SEM images of cross sectioned PMMA foams nucleated by $120 \mathrm{SiO}_{2}$-RP NPs. The multiple cells nucleation events were observed for some of the particles.
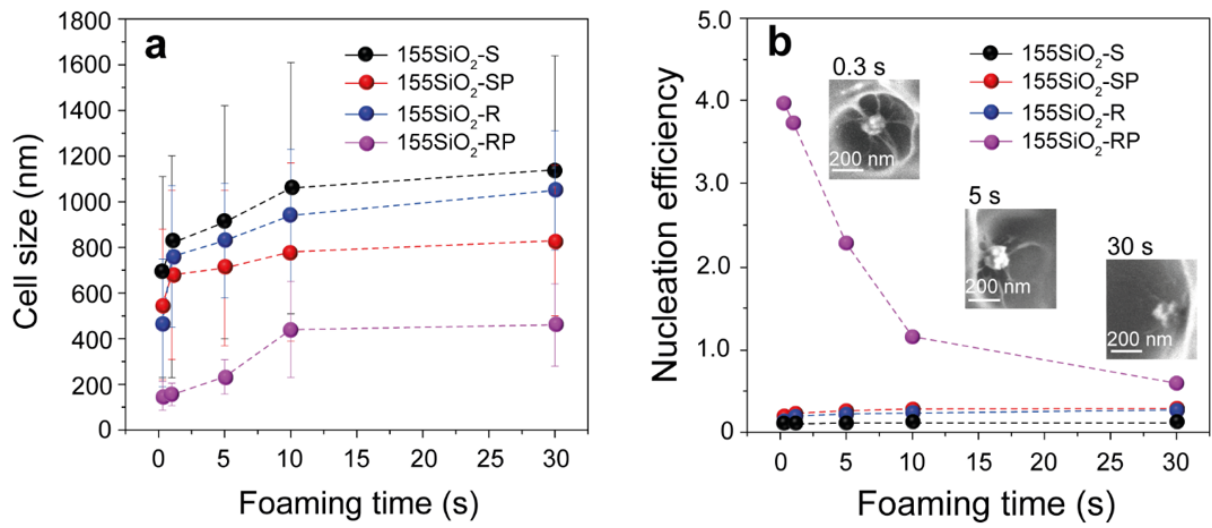

Figure S5.5 (a) Foam cell size and (b) cell nucleation efficiency as a function of foaming time for PMMA foams nucleated by $155 \mathrm{SiO}_{2}-\mathrm{S}, 155 \mathrm{SiO}_{2}-\mathrm{SP}, 155 \mathrm{SiO}_{2}-\mathrm{R}$ and $155 \mathrm{SiO}_{2}-\mathrm{RP}$ $N P$ s. The used saturation pressure and foaming temperature were 55 bar and $40{ }^{\circ} \mathrm{C}$, respectively.

The cell size and cell nucleation efficiency for PMMA foams nucleated by $155 \mathrm{SiO}_{2}$ - 
$\mathrm{S}, 155 \mathrm{SiO}_{2}-\mathrm{SP}, 155 \mathrm{SiO}_{2}-\mathrm{R}$ and $155 \mathrm{SiO}_{2}-\mathrm{RP}$ NPs were determined as a function of the foaming time. The results are shown in Figure S5.5. It is obvious that regardless of the type of NP used cell size of the corresponding PMMA foams increases as a function of foaming time up to 10 seconds after which it reaches a plateau value with a size value that depends on the nucleating particle type used.

The cell nucleation efficiency of PMMA foams containing $155 \mathrm{SiO}_{2}-\mathrm{SP}$ NPs is higher compared to those containing $155 \mathrm{SiO}_{2}-\mathrm{S}$ NPs for the same foaming time (as shown in Figure $\mathrm{S} 5.5 \mathrm{~b}$ ), which is ascribed to the high $\mathrm{CO}_{2}$-philicity and low surface energy of the thin PDMS shell of the $155 \mathrm{SiO}_{2}-\mathrm{SP} N P s .{ }^{56}$ Interestingly, the $\mathrm{SiO}_{2}-\mathrm{R}$ NPs, i.e. $155 \mathrm{SiO}_{2}-\mathrm{R}$ $\mathrm{NPs}$, show a higher cell nucleation efficiency compared to that of $155 \mathrm{SiO}_{2}-\mathrm{S} \mathrm{NPs}$, as well. This is ascribed to the presence of cavities on the $\mathrm{SiO}_{2}-\mathrm{R} \mathrm{NP}$ surfaces, resulting in energetically favorable cell nucleation. ${ }^{40}$ The nucleation efficiency of $155 \mathrm{SiO}_{2}-\mathrm{RP}$ decreases upon increasing the foaming time. This is due to the observed cell coalescence occurring during the early stages of foaming (see Figure 5.5c-f). Notably, the nucleation efficiency of $155 \mathrm{SiO}_{2}$-RP NPs is well above 1 within the first 10 seconds of foaming, which is ascribed to the multiple cell nucleation events occurring per nucleating $155 \mathrm{SiO}_{2}$ RP NPs. The excellent nucleation performance of $155 \mathrm{SiO}_{2}-\mathrm{RP}$ NPs is ascribed to the energetically favorable cell nucleation from the particles surface cavities combined with the presence of $\mathrm{a} \mathrm{CO}_{2}$ philic PDMS layer around the $155 \mathrm{SiO}_{2}-\mathrm{RP}$ NPs. ${ }^{40-42}$

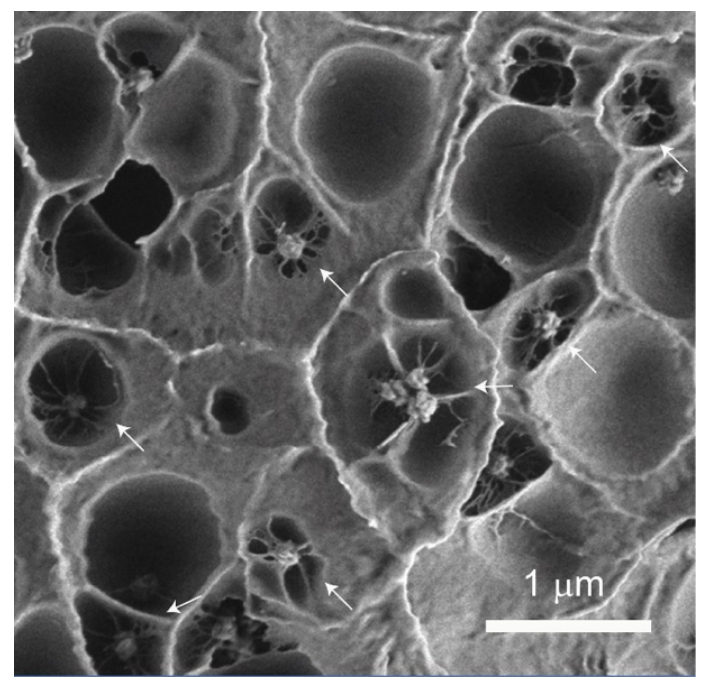

Figure S5.6 SEM images of cross sectioned polystyrene foams nucleated by $150 \mathrm{SiO}_{2}-\mathrm{RP}$ NPS. The saturation pressure, foaming temperature and foaming time were 55 bar, 100 ${ }^{\circ} \mathrm{C}$ and 1 second, respectively. The white arrows point towards the particles showing multiple cell nucleation events per particle. 

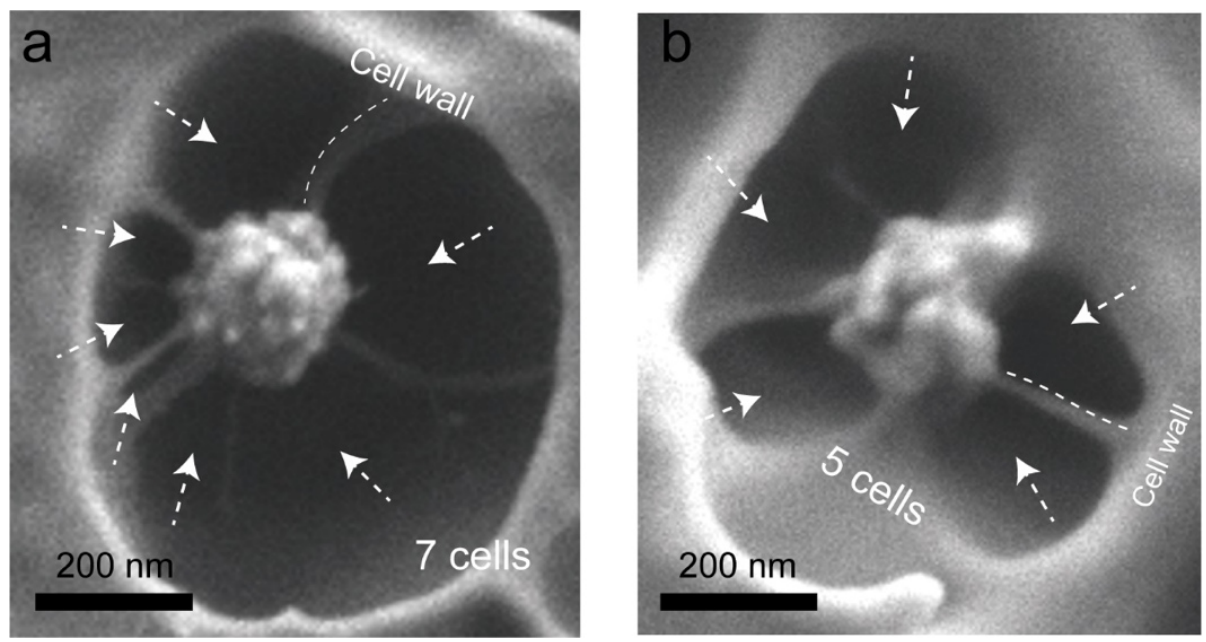

Figure S5.7 SEM image showing multiple nucleating events at individual 200SiO $2-R P$ NPs. The white arrows point at the cells we included in the calculation of the cell density. We counted cells only when the cell walls were clearly visible.

\subsection{References}

(1) Costeux, S. CO $\mathrm{CO}_{2}$-Blown Nanocellular Foams . Journal of Applied Polymer Science 2014, 131 (23), 10.1002/app.41293.

(2) Mills, N. J. Handbook of Polymeric Foams and Foam Technology. Polymer 1993, 34 (10), 2237.

(3) Forest, C.; Chaumont, P.; Cassagnau, P.; Swoboda, B.; Sonntag, P. Polymer Nano-Foams for Insulating Applications Prepared from $\mathrm{CO}_{2}$ Foaming. Progress in Polymer Science 2015, 41 (C), 122-145.

(4) Liu, S.; Duvigneau, J.; Vancso, G. J. Nanocellular Polymer Foams as Promising High Performance Thermal Insulation Materials. European Polymer Journal 2015, 65, 33-45.

(5) Notario, B.; Pinto, J.; Rodriguez-Perez, M. A. Nanoporous Polymeric Materials: A New Class of Materials with Enhanced Properties. Progress in Materials Science 2016, 78-79, 93-139.

(6) Lee, L. J.; Zeng, C.; Cao, X.; Han, X.; Shen, J.; Xu, G. Polymer Nanocomposite Foams. Composites Science and Technology 2005, 65 (15-16 SPEC. ISS.), 23442363.

(7) Notario, B.; Pinto, J.; Solorzano, E.; de Saja, J. A.; Dumon, M.; Rodríguez-Pérez, M. A. Experimental Validation of the Knudsen Effect in Nanocellular Polymeric Foams. Polymer 2015, 56, 57-67.

(8) Pinto, J.; Notario, B.; Verdejo, R.; Dumon, M.; Costeux, S.; Rodriguez-Perez, M. A. Molecular Confinement of Solid and Gaseous Phases of Self-Standing Bulk Nanoporous Polymers Inducing Enhanced and Unexpected Physical Properties. 
Polymer 2017, 113, 27-33.

(9) Notario, B.; Pinto, J.; Rodríguez-Pérez, M. A. Towards a New Generation of Polymeric Foams: PMMA Nanocellular Foams with Enhanced Physical Properties. Polymer 2015, 63, 116-126.

(10) Lee, S. T.; Ramesh, N. S. Polymeric Foams: Mechanisms and Materials; 2004.

(11) Li, L.; Shen, X.; Hong, S. W.; Hayward, R. C.; Russell, T. P. Fabrication of CoContinuous Nanostructured and Porous Polymer Membranes: Spinodal Decomposition of Homopolymer and Random Copolymer Blends. Angewandte Chemie - International Edition 2012, 51 (17), 4089-4094.

(12) Zhang, X.; Chang, D.; Liu, J.; Luo, Y. Conducting Polymer Aerogels from Supercritical $\mathrm{CO}_{2}$ Drying PEDOT-PSS Hydrogels. Journal of Materials Chemistry 2010, 20 (24), 5080-5085.

(13) Hedrick, J. L.; Miller, R. D.; Hawker, C. J.; Carter, K. R.; Volksen, W.; Yoon, D. Y.; Trollsås, M. Templating Nanoporosity in Thin-Film Dielectric Insulators. Advanced Materials 1998, 10 (13), 1049-1053.

(14) Park, S. H.; Xia, Y. Macroporous Membranes with Highly Ordered and ThreeDimensionally Interconnected Spherical Pores. Advanced Materials 1998, 10 (13), 1045-1048.

(15) du Fresne Von Hohenesche, C.; Schmidt, D. F.; Schädler, V. Nanoporous Melamine - Formaldehyde Gels by Microemulsion Templating. Chemistry of Materials 2008, 20 (19), 6124-6129.

(16) Bledzki, A. K.; Faruk, O. Effects of the Chemical Foaming Agents, Injection Parameters, and Melt-Flow Index on the Microstructure and Mechanical Properties of Microcellular Injection-Molded Wood-Fiber/Polypropylene Composites. Journal of Applied Polymer Science 2005, 97 (3), 1090-1096.

(17) Zeng, C.; Han, X.; Lee, L. J.; Koelling, K. W.; Tomasko, D. L. Polymer-Clay Nanocomposite Foams Prepared Using Carbon Dioxide. Advanced Materials 2003, 15 (20), 1743-1747.

(18) Park, C. B.; Behravesh, A. H.; Venter, R. D. Low Density Microcellular Foam Processing in Extrusion Using $\mathrm{CO}_{2}$. Polymer Engineering and Science 1998, 38 (11), 1812-1823.

(19) Tomasko, D. L.; Li, H.; Liu, D.; Han, X.; Wingert, M. J.; Lee, L. J.; Koelling, K. W. A Review of $\mathrm{CO}_{2}$ Applications in the Processing of Polymers. Industrial and Engineering Chemistry Research 2003, 42 (25), 6431-6456.

(20) Costeux, S.; Zhu, L. Low Density Thermoplastic Nanofoams Nucleated by Nanoparticles. Polymer 2013, 54 (11), 2785-2795.

(21) Costeux, S.; Bunker, S. P.; Jeon, H. K. Homogeneous Nanocellular Foams from Styrenic-Acrylic Polymer Blends. Journal of Materials Research 2013, 28 (17), 2351-2365.

(22) Liao, Z. E.; Yeh, S. K.; Chu, C. C.; Tseng, T. W. Critical Parameters of Generating PMMA Nanocellular Foam. Proceedings of the Annual Technical Conference 2016, 1773-1778.

(23) Tammaro, D.; Astarita, A.; di Maio, E.; Iannace, S. Polystyrene Foaming at High Pressure Drop Rates. Industrial and Engineering Chemistry Research 2016, 55 (19), 5696-5701.

(24) Pinto, J.; Dumon, M.; Pedros, M.; Reglero, J.; Rodriguez-Perez, M. A. 
Nanocellular $\mathrm{CO}_{2}$ Foaming of PMMA Assisted by Block Copolymer Nanostructuration. Chemical Engineering Journal 2014, 243, 428-435.

(25) Li, L.; Nemoto, T.; Sugiyama, K.; Yokoyama, H. $\mathrm{CO}_{2}$ Foaming in Thin Films of Block Copolymer Containing Fluorinated Blocks. Macromolecules 2006, 39 (14), 4746-4755.

(26) Spitael, P.; Macosko, C. W.; McClurg, R. B. Block Copolymer Micelles for Nucleation of Microcellular Thermoplastic Foams. Macromolecules 2004, 37 (18), 6874-6882.

(27) Yokoyama, H.; Sugiyama, K. Nanocellular Structures in Block Copolymers with $\mathrm{CO}_{2}$-Philic Blocks Using $\mathrm{CO}_{2}$ as a Blowing Agent: Crossover from Micro- to Nanocellular Structures with Depressurization Temperature. Macromolecules 2005, 38 (25), 10516-10522.

(28) Liu, S.; Zoetebier, B.; Hulsman, L.; Zhang, Y.; Duvigneau, J.; Vancso, G. J. Nanocellular Polymer Foams Nucleated by Core-Shell Nanoparticles. Polymer 2016, 104, 22-30.

(29) Ji, G.; Zhai, W.; Lin, D.; Ren, Q.; Zheng, W.; Jung, D. W. Microcellular Foaming of Poly(Lactic Acid)/Silica Nanocomposites in Compressed $\mathrm{CO}_{2}$ : Critical Influence of Crystallite Size on Cell Morphology and Foam Expansion. Industrial and Engineering Chemistry Research 2013, 52 (19), 6390-6398.

(30) Zhai, W.; Yu, J.; Wu, L.; Ma, W.; He, J. Heterogeneous Nucleation Uniformizing Cell Size Distribution in Microcellular Nanocomposites Foams. Polymer 2006, 47 (21), 7580-7589.

(31) Yuan, H.; Xiong, Y.; Luo, G.; Shen, Q.; Zhang, L. The Influence of in Situ Synthesized Nanoparticles on Microstructure and Compression Properties of Polymer Foams during Supercritical Carbon Dioxide Foaming. Journal of Applied Polymer Science 2017, 134 (14).

(32) Rende, D.; Schadler, L. S.; Ozisik, R. Controlling Foam Morphology of Poly(Methyl Methacrylate) via Surface Chemistry and Concentration of Silica Nanoparticles and Supercritical Carbon Dioxide Process Parameters. Journal of Chemistry 2013, 2013, 864926.

(33) Liberman, A.; Mendez, N.; Trogler, W. C.; Kummel, A. C. Synthesis and Surface Functionalization of Silica Nanoparticles for Nanomedicine. Surface Science Reports 2014, 69 (2-3), 132-158.

(34) Bagwe, R. P.; Hilliard, L. R.; Tan, W. Surface Modification of Silica Nanoparticles to Reduce Aggregation and Nonspecific Binding. Langmuir 2006, $22(9), 4357-4362$.

(35) Yang, J.; Sang, Y.; Chen, F.; Fei, Z.; Zhong, M. Synthesis of Silica Particles Grafted with Poly(Ionic Liquid) and Their Nucleation Effect on Microcellular Foaming of Polystyrene Using Supercritical Carbon Dioxide. Journal of Supercritical Fluids 2012, 62, 197-203.

(36) Goren, K.; Chen, L.; Schadler, L. S.; Ozisik, R. Influence of Nanoparticle Surface Chemistry and Size on Supercritical Carbon Dioxide Processed Nanocomposite Foam Morphology. Journal of Supercritical Fluids 2010, 51(3), 420-427.

(37) Siripurapu, S.; DeSimone, J. M.; Khan, S. A.; Spontak, R. J. Low-Temperature, Surface-Mediated Foaming of Polymer Films. Advanced Materials 2004, 16 (12), 989-994. 
(38) Miller, M. B.; Luebke, D. R.; Enick, R. M. CO2-Philic Oligomers as Novel Solvents for $\mathrm{CO}_{2}$ Absorption. Energy and Fuels 2010, 24 (11), 6214-6219.

(39) Wang, H.; Li, W. Selective Ultrasonic Foaming of Polymer for Biomedical Applications. Journal of Manufacturing Science and Engineering, Transactions of the ASME 2008, 130 (2), 0210041-0210049.

(40) Liu, Q.; Zhu, Y.; Yang, G.; Yang, Q. Nucleation Thermodynamics inside Micro/Nanocavity. Journal of Materials Science and Technology 2008, 24 (2), 183-186.

(41) Qian, M.; Ma, J. The Characteristics of Heterogeneous Nucleation on Concave Surfaces and Implications for Directed Nucleation or Surface Activity by Surface Nanopatterning. Journal of Crystal Growth 2012, 355 (1), 73-77.

(42) Maksimov, A. O.; Kaverin, A. M.; Baidakov, V. G. Heterogeneous Vapor Bubble Nucleation on a Rough Surface. Langmuir 2013, 29 (12), 3924-3934.

(43) Tsai, H. J.; Lee, Y. L. Facile Method to Fabricate Raspberry-like Particulate Films for Superhydrophobic Surfaces. Langmuir 2007, 23 (25), 12687-12692.

(44) Cano, M.; de la Cueva-Méndez, G. Self-Assembly of a Superparamagnetic Raspberry-like Silica/Iron Oxide Nanocomposite Using Epoxy-Amine Coupling Chemistry. Chemical Communications 2015, 51 (17), 3620-3622.

(45) Ming, W.; Wu, D.; van Benthem, R.; de With, G. Superhydrophobic Films from Raspberry-like Particles. Nano Letters 2005, 5 (11), 2298-2301.

(46) Puretskiy, N.; Ionov, L. Synthesis of Robust Raspberry-like Particles Using Polymer Brushes. Langmuir 2011, 27 (6), 3006-3011.

(47) Wang, J.; Yang, X. Raspberry-like Polymer/Silica Core-Corona Composite by Self-Assemble Heterocoagulation Based on a Hydrogen-Bonding Interaction. Colloid and Polymer Science 2008, 286 (3), 283-291.

(48) Mammen, L.; Deng, X.; Untch, M.; Vijayshankar, D.; Papadopoulos, P.; Berger, R.; Riccardi, E.; Leroy, F.; Vollmer, D. Effect of Nanoroughness on Highly Hydrophobic and Superhydrophobic Coatings. Langmuir 2012, 28 (42), 1500515014.

(49) Yuhong, Z.; Qichao, Z.; Xingwang, S.; Qingqiong, T.; Min, C.; Limin, W. Preparation of Raspberry-like Polymer/Silica Nanocomposite Microspheres via Emulsifier-Free Polymerization in Water/Acetone Media. Journal of Colloid and Interface Science 2009, 336 (2), 544-550.

(50) Du, X.; Liu, X.; Chen, H.; He, J. Facile Fabrication of Raspberry-like Composite Nanoparticles and Their Application as Building Blocks for Constructing Superhydrophilic Coatings. Journal of Physical Chemistry C 2009, 113 (21), 9063-9070.

(51) Zhang, T.; Zhang, Q.; Ge, J.; Goebl, J.; Sun, M.; Yan, Y.; Liu, Y. S.; Chang, C.; Guo, J.; Yin, Y. A Self-Templated Route to Hollow Silica Microspheres. Journal of Physical Chemistry C 2009, 113 (8), 3168-3175.

(52) Du, X.; He, J. A Self-Templated Etching Route to Surface-Rough Silica Nanoparticles for Superhydrophobic Coatings. ACS Applied Materials and Interfaces 2011, 3 (4), 1269-1276.

(53) Zhang, T.; Ge, J.; Hu, Y.; Zhang, Q.; Aloni, S.; Yin, Y. Formation of Hollow Silica Colloids through a Spontaneous Dissolution-Regrowth Process. Angewandte Chemie - International Edition 2008, 47 (31), 5806-5811. 
(54) Musić, S.; Filipović-Vinceković, N.; Sekovanić, L. Precipitation of Amorphous $\mathrm{SiO}_{2}$ Particles and Their Properties. Brazilian Journal of Chemical Engineering 2011, 28 (1), 89-94.

(55) Kim, H.; Kim, H. G.; Kim, S.; Kim, S. S. PDMS-Silica Composite Membranes with Silane Coupling for Propylene Separation. Journal of Membrane Science 2009, 344 (1-2), 211-218.

(56) Liu, S.; Eijkelenkamp, R.; Duvigneau, J.; Vancso, G. J. Silica-Assisted Nucleation of Polymer Foam Cells with Nanoscopic Dimensions: Impact of Particle Size, Line Tension, and Surface Functionality. ACS Applied Materials and Interfaces 2017, 9 (43), 37929-37940.

(57) Thommes, M. Physical Adsorption Characterization of Nanoporous Materials. Chemie-Ingenieur-Technik 2010, 82 (7), 1059-1073.

(58) Thommes, M.; Cychosz, K. A. Physical Adsorption Characterization of Nanoporous Materials: Progress and Challenges. Adsorption 2014, 20 (2-3), 233250.

(59) Rouquerol, J.; Rouquerol, F.; Llewellyn, P.; Maurin, G.; Sing, K. S. W. (2013). Adsorption by Powders and Porous Solids: Principles, Methodology and Applications: Second Edition. Academic Press.

(60) Liu, S.; Pandey, A.; Duvigneau, J.; Vancso, J.; Snoeijer, J. H. Size-Dependent Submerging of Nanoparticles in Polymer Melts: Effect of Line Tension. Macromolecules 2018, 51 (7), 2411-2417.

(61) Liu, S. (2017). Designer Nanoparticles as Efficient Nucleation Agents in Polymer Foaming. University of Twente. https://doi.org/10.3990/1.9789036544023

(62) Jones, W. M.; Isaac, P. J.; Phillips, D. The Adsorption of Carbon Dioxide and Nitrogen at High Pressures by Porous Plugs of Lampblack. Transactions of the Faraday Society 1959, 55 (0), 1953-1958.

(63) Ally, J.; Molla, S.; Mostowfi, F. Condensation in Nanoporous Packed Beds. Langmuir 2016, 32 (18), 4494-4499.

(64) Ullah, R.; Atilhan, M.; Diab, A.; Deniz, E.; Aparicio, S.; Yavuz, C. T. Synthesis, Characterization and Evaluation of Porous Polybenzimidazole Materials for $\mathrm{CO}_{2}$ Adsorption at High Pressures. Adsorption 2016, 22 (2), 247-260.

(65) Fujiki, J.; Yamada, H.; Yogo, K. Enhanced Adsorption of Carbon Dioxide on Surface-Modified Mesoporous Silica-Supported Tetraethylenepentamine: Role of Surface Chemical Structure. Microporous and Mesoporous Materials 2015, 215 , 76-83.

(66) Fujiki, J.; Yogo, K. The Increased $\mathrm{CO}_{2}$ Adsorption Performance of ChitosanDerived Activated Carbons with Nitrogen-Doping. Chemical Communications 2016, 52 (1), 186-189.

(67) Sanz-Pérez, E. S.; Arencibia, A.; Sanz, R.; Calleja, G. An Investigation of the Textural Properties of Mesostructured Silica-Based Adsorbents for Predicting $\mathrm{CO}_{2}$ Adsorption Capacity. RSC Advances 2015, 5 (125), 103147-103154.

(68) Kumar, V.; Suh, N. P. A Process for Making Microcellular Thermoplastic Parts. Polymer Engineering \& Science 1990, 30 (20), 1323-1329. 



\section{Chapter}

\section{Fluorescent Polyethylene by In-Situ Facile Synthesis of Carbon Quantum Dots Facilitated by Silica Nanoparticle Agglomerates}

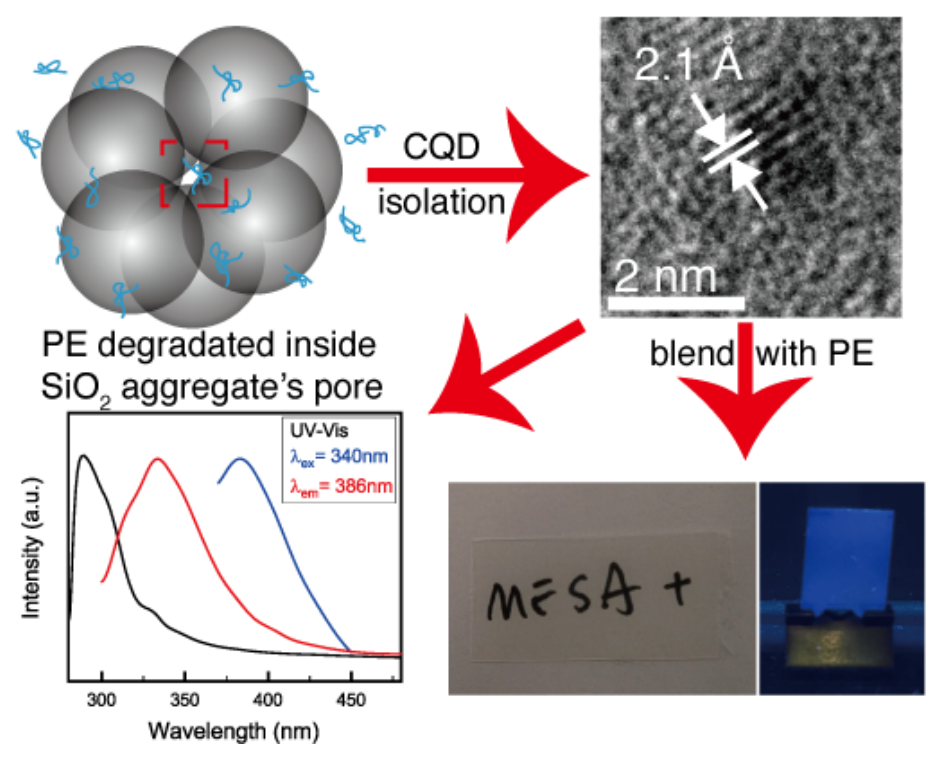

We describe an in-situ facile synthetic approach to prepare carbon quantum dot (CQD) fluorescent markers (FMs) for polyethylene in the presence of agglomerated silica nanoparticles $\left(\mathrm{SiO}_{2} \mathrm{NPs}\right)$, under mild reaction conditions. First $\mathrm{SiO}_{2} \mathrm{NPs}$, prepared by 
the Stöber method, were dispersed in toluene. This dispersion was added to a polyethylene (PE) solution in toluene. After heating (either in air or under Ar) a fluorescent mixture was obtained. After drying, PE films were obtained by compression molding, which showed strong blue fluorescence, attributed to CQDs. $\mathrm{SiO}_{2} \mathrm{NPs}_{\text {loading }}$ values varied between 0.5 and 4 wt \%. Subsequent to isolation, the fluorescent CQDs were characterized by TEM, UV-VIS and fluorescence spectroscopy. TEM imaging unveiled a lattice spacing value of $0.21 \mathrm{~nm}$ of the isolated fluorescent particles that is typical for (100) graphite plane lattice spacing in CQDs. UV spectroscopy and fluorescence measurements show characteristic absorption and excitation spectra for aromatic core and oxidized surface defects typically observed for CQDs. The emission maximum for PE/CQD samples increased from $394 \mathrm{~nm}$ to $408 \mathrm{~nm}$ when the reaction temperature was decreased from $110^{\circ} \mathrm{C}$ to $90^{\circ} \mathrm{C}$, which is attributed to increasing oxygen content in the reaction mixture upon decreasing the reaction temperature. When the reaction was performed under Ar, the PE/CQD samples emitted in the ultraviolet region (286 nm). Finally, we demonstrated that PE samples marked with CQDs can be easily visually identified upon irradiating with $367 \mathrm{~nm}$ light. Thus the marked PE can be used e.g. as a labelling ingredient in master batches for component identification, and in recycling.

The contents of this Chapter will be published as: Yin, S.; Duvigneau, J.; Vancso, G. J. Fluorescent Polyethylene by In-Situ Facile Synthesis of Carbon Quantum Dots Facilitated by Silica Nanoparticle Agglomerates. Accepted. 


\subsection{Introduction}

To decrease the environmental impact of plastic waste and consumption of fossil resources employed in the production of plastics, recycling plastic waste is an essential practice. However, plastic sorting efficiency and component identification remain a technological challenge. ${ }^{1-3}$ To increase the recycling rate for plastic waste, one needs to improve the sorting accuracy and speed. To this end, adding fluorescent markers (FMs) to enable high-efficiency sorting is one of the most promising approaches.

Currently, there are three main approaches to plastic waste management. These are landfilling, incineration, and chemical/mechanical recycling. ${ }^{3}$ In general, plastic waste streams are treated with other municipal waste streams for landfilling and incineration. More than $80 \%$ of the waste plastic is disposed in the landfill. ${ }^{3}$ Due to plastics' usually high resistance to degradation, it can take more than 50 years for a piece of plastic to degrade after being buried. ${ }^{4,5}$ Degradation of landfilled plastic waste can damage the environment by, e.g., releasing large amounts of greenhouse gasses $\left(\mathrm{CO}_{2}\right)$, accelerating global warming, and forming microplastics/nanoplastics that may eventually accumulate in humans. ${ }^{6,7}$ Besides these environmental concerns, landfilling is getting increasingly expensive in highly-populated areas, like in Western Europe and in Southern China. ${ }^{8}$ Incineration is also a standard method for plastic waste management. ${ }^{3}$ Its most significant advantages are that upon incineration, the waste volume is reduced and that simultaneously, the heat of combustion can be used for electricity generation. In 2015, approximately $12 \%$ of the global plastic waste was incinerated. ${ }^{9}$ However, the toxic gases released by incineration pose a significant health and environmental concern, especially when the incineration plants are operated in densely populated areas. ${ }^{10}$ Furthermore, the incineration of plastic waste is accompanied by a relatively high $\mathrm{CO}_{2}$ emission. Incineration of $1 \mathrm{~kg}$ plastic waste can release up to $4.6 \mathrm{~kg}$ of $\mathrm{CO}_{2}$, which for $1 \mathrm{~kg}$ municipal solid waste (MSW) is only $1.2 \mathrm{~kg} .{ }^{11}$ Typically, MSW contains approximately $10-12 \%$ of plastic. Thus sorting plastic waste from MSW before incineration will substantially decrease greenhouse gas emissions. ${ }^{10}$

Compared to landfilling and incineration, recycling is an eco-friendlier way to deal with plastic waste. ${ }^{11-13}$ Recycling plastic reduces the above-mentioned environmental issues and decreases the required amount of fossil fuel to produce new plastic by reducing the demand for virgin materials. It is widely accepted that recycling plastics is an essential step towards creating a circular economy. ${ }^{14}$ There are two main approaches for recycling plastics, i.e. by using chemical or mechanical methods. ${ }^{2}$ Depending on the composition of the plastics to recycle, each method has its strengths and weaknesses. ${ }^{1}$ 
The sorting of plastic waste typically consists of three steps. ${ }^{1}$ First, Fourier transform near-infrared (FTNR) spectroscopy and a color recognition camera are used to pre-sort various plastic types from waste streams. The second step consists of milling the plastic waste into small pieces and removing the non-plastic residuals. The final step is to further sort the milled plastics by type. Various techniques are in place for sorting plastic waste streams, like sink/float separation, ${ }^{15}$ electrostatic separation, ${ }^{16}$ air elutriation, ${ }^{17}$ froth flotation, ${ }^{18}$ and selective degradation. ${ }^{19}$ Each of the above-mentioned techniques has its strengths and weaknesses; for more details, the interested reader is directed to references 20,21 .

The use of FMs, as an accurate and cost-effective approach to identify and sort specific polymers from plastic waste streams, has received considerable attention in recent years. ${ }^{21-25}$ FMs can be incorporated in polymer matrices via masterbatches, e.g., by extrusion or injection molding. They can even be applied as a thin fluorescent coating onto the finished plastic products or intermediates. ${ }^{26,27}$ For instance, Massardier et al. reported the incorporation of $0.1 \mathrm{wt} \%$ of lanthanide complex particles into a polypropylene (PP) matrix under a high shear rate $(\mathrm{N}=800 \mathrm{rpm})$ to prepare a fluorescent polypropylene that is detectable with UV-ray fluorescence spectrometry within 10 milliseconds. ${ }^{23}$ Langhals et al. reported using organic dyes, i.e. perylene ester, perylene carboxylic bisimide, and terylene carboxylic bisimide FMs to identify polymers by measuring the time constant of autofluorescence decay values. ${ }^{25}$ They also demonstrated that the polymer type (styrene-polyacrylonitrile copolymer, polyoxymethylene, or polyamide) alters the fluorescence lifetime values of the incorporated markers. This observation opened the path towards using the difference in the time constant values of the autofluorescence decay in sorting of different types of plastics. Recently, Woidasky et al. reported adding oxide crystals, doped with rare earth elements, as up-conversion inorganic FMs to the polymer matrix for marking and identification. ${ }^{28}$ By utilizing the up-conversion fluorescence of the FMs, the authors were able to generate photoluminescence (PL) in the visible light range upon near IR (NIR) excitation and, as such, eliminate the influence of background emission from plastics and additives.

Despite promises, there are numerous open challenges when considering the use of fluorescent particles/dyes at industrially relevant scales. For instance, fluorescent particles/dyes usually suffer from high cost, toxicity, and poor photostability. ${ }^{29-32}$ These drawbacks currently limit their use as FM labeled plastics on a large scale. Thus, if progress is to be made in this area, additional efforts are needed. Keeping this in mind, we decided to tackle the problem of loading polyethylene (PE) by carbon quantum dots (hereinafter CQDs) and explored facile reaction routes to obtain fluorescent labelled PE. 
CQDs are considered as a viable alternative for fluorescent dyes and inorganic QDs because of their low toxicity, low cost, good solubility, and high photostability. ${ }^{33} \mathrm{~A}$ variety of techniques have been deployed to synthesize CQDs. These methods can be classified into two categories, i.e. top-down and bottom-up approaches. ${ }^{34-36}$

The 'top-down' method usually involves chemical, electrochemical and physical approaches to break down large carbon-based materials, i.e. carbon soot, carbon nanotubes, graphite, etc. ${ }^{37,38}$ The 'bottom-up' approach typically utilizes solvothermal/hydrothermal carbonization, laser ablation, electrochemical oxidation, microwave irradiation, or pyrolysis to grow CQDs from organic molecules. ${ }^{39}$ Many organic compounds have been reported to be used to synthesize CQDs via the bottom-up method, for instance, polybasic acid, glucose, sucrose, glycol, glycerol, chitosan. ${ }^{40-44}$ Among the techniques used to synthesize CQDs, solvothermal/hydrothermal carbonization is considered one of the simplest, most direct, and most efficient pathways. ${ }^{43}$ However, the complicated chemical reactions, time-consuming purification, and harsh synthesis conditions pose serious challenges for large-scale applications. ${ }^{36,45}$

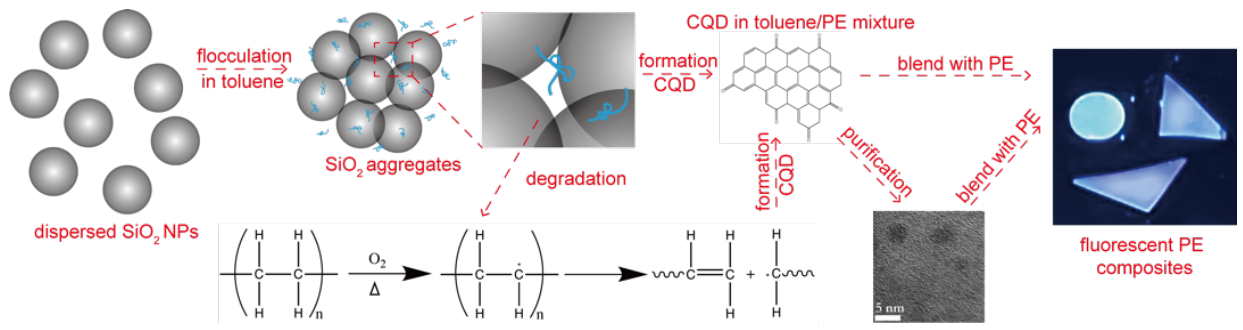

Figure 6.1 Schematic of CQD formation through thermal PE degradation inside the cavities/pores of silica nanoparticle aggregates. The method does not require extensive particle purification; upon removing the solvent, the particles are readily used as FMs in $P E$.

In this Chapter we introduce a facile 'bottom-up' route that uses silica nanoparticles $\left(\mathrm{SiO}_{2} \mathrm{NPs}\right)$ as enablers to fabricate fluorescent CQDs from polyethylene (PE). The reaction scheme is captured in Figure 6.1. One of the most beneficial features of this route is that upon removing the solvent, the CQD, silica, and PE mixture is present in a concentrated FM blend, which can be used as a masterbatch to add to (virgin) PE. Hence extensive and costly CQD collection and purification are not required. This opens new avenues to the widespread applicability of CQDs as FM for plastic waste recycling, which we will demonstrate in the last part of this Chapter. 


\subsection{Results and Discussion}

\subsection{1 $\mathrm{SiO}_{2}$ Synthesis and Characterization}

First, we employed a modified Stöber procedure to synthesize $\mathrm{SiO}_{2} \mathrm{NPs}^{46}{ }^{46}$ Figure6.2a and Figure6.2b show a scanning electron microscope (SEM) image and the size distribution of the $\mathrm{SiO}_{2} \mathrm{NPs}$, respectively. The nearly spherical $\mathrm{SiO}_{2} \mathrm{NPs}$ have an average particle size of $114 \pm 11 \mathrm{~nm}$ and most of the particles have a diameter between 100 and $130 \mathrm{~nm}$. Figure6.2c shows a Fourier-transform infrared (FTIR) absorbance spectrum of the $\mathrm{SiO}_{2}$ NPs. In the FTIR spectrum, the $\mathrm{SiO}_{2}$ signature absorption bands ascribed to the asymmetric vibration of $\mathrm{Si}-\mathrm{O}$, the asymmetric vibration of $\mathrm{Si}-\mathrm{OH}$, and the symmetric vibration of Si-O are clearly observed at $1063 \mathrm{~cm}^{-1}, 940 \mathrm{~cm}^{-1}$, and $803 \mathrm{~cm}^{-1}$, respectively. The broad absorption band with a maximum at $3431 \mathrm{~cm}^{-1}$ and the small peak located at $1635 \mathrm{~cm}^{-1}$ are ascribed to O-H stretching absorbances by Si-OH bonds and to physically absorbed water. ${ }^{47}$ The small absorption bands located at $2980 \mathrm{~cm}^{-1}$ and $1452 \mathrm{~cm}^{-1}$ indicate the presence of $\mathrm{CH}_{2} / \mathrm{CH}_{3}$, which is attributed to residual ethoxy moieties. ${ }^{47}$
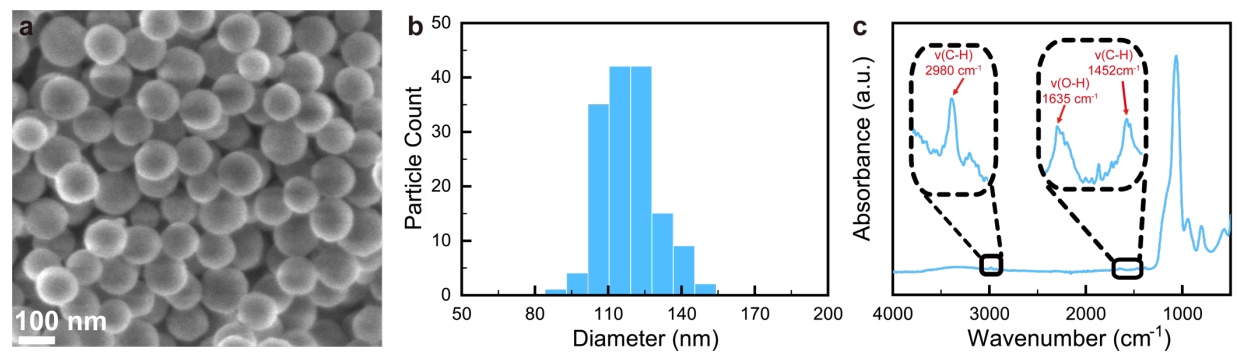

Figure 6.2 a) SEM image and b) size distribution of $\mathrm{SiO}_{2} \mathrm{NPs}_{\text {used }}$ in this work. c) Single reflection ATR-FTIR absorbance spectrum of the as-prepared $\mathrm{SiO}_{2} \mathrm{NPS}$.

\subsection{2 $\mathrm{PE} / \mathrm{SiO}_{2}$ Composites and Fluorescence Microscopy}

In Figure 6.3, fluorescent microscope images $\left(\lambda_{\mathrm{ex}} 360-370 \mathrm{~nm}\right.$ and $\left.\lambda_{\mathrm{em}}>460 \mathrm{~nm}\right)$ of 2.5-PE/ $\mathrm{SiO}_{2}$ thin films obtained by employing varying reaction times up to $72 \mathrm{~h}$ are shown. From this figure, it is clear that upon increasing the reaction time, the observed fluorescent emission intensity increases (see also later). Additionally, for separate $\mathrm{PE} /$ toluene and $\mathrm{SiO}_{2} /$ toluene samples heated for 24 hours in air at $110^{\circ} \mathrm{C}$, no fluorescence was observed. Thus, we conclude that the combination of $\mathrm{SiO}_{2} \mathrm{NPs}$ and $\mathrm{PE}$ together is required for the reaction that results in fluorescent material. In the following sections, we will first confirm that CQDs are formed and explain the role of the $\mathrm{SiO}_{2} \mathrm{NPs}$ in the reaction. In the last part of this work, we show that modest shifts in the emission wavelengths are obtained upon changing the reaction conditions (from air to Ar). 

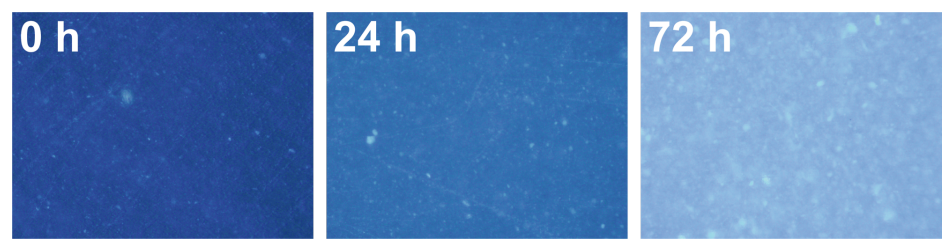

Figure 6.3 Fluorescent microscopy images of 2.5-PE/SiO ${ }_{2}$ thin films prepared as a function of the reaction time up to 72 hours in air.

\subsubsection{The Origin of the Observed Fluorescent: CQD Isolation and Characterization}

During preparation, as mentioned, liquid nitrogen quenching of the toluene solution containing 2.5-PE/ $\mathrm{SiO}_{2}$ and CQDs was used to suppress $\mathrm{PE}$ crystallization and leave the particles $\left(\mathrm{SiO}_{2} \mathrm{NPs}\right.$ and CQDs) in the toluene phase. Following the removal of the residual $\mathrm{PE}$ and subsequently the $\mathrm{SiO}_{2}$ NPs by filtration, the residual toluene fraction was dried to isolate the fluorophores. Subsequently, small quantities of a yellowish paste were formed at the bottom of the container. This paste was used to prepare samples for transmission electron microscope (TEM) analysis. Figure 6.4a shows an TEM image of the residual paste, revealing crystalline nanoparticles (dark, circular objects). The arrows in Figure 6.4a point at single fluorophores. From zoomed-in images (such as shown in the top right inset) and following a Fast Fourier Transform (FFT) analysis of the TEM intensities (bottom right inset), a lattice spacing of $2.1 \AA$ for the nanoparticles was determined. This value agrees well with the (100) plane graphite lattice spacing typically observed for CQDs. ${ }^{48-51}$ While we expected clear TEM images of the CQDs, the particle edges appear slightly blurred. We attribute this to the presence of residual polymer that the isolation process did not remove, combined with curvature effects. The residual polymer promoted particle agglomeration during TEM sample preparation. The average diameter for the CQDs was $2.3 \pm 0.5 \mathrm{~nm}$, which agrees with the particle height measured by atomic force microscope (AFM) $(2.2 \pm 0.7 \mathrm{~nm}$, see Figure $6.4 \mathrm{c})$ 

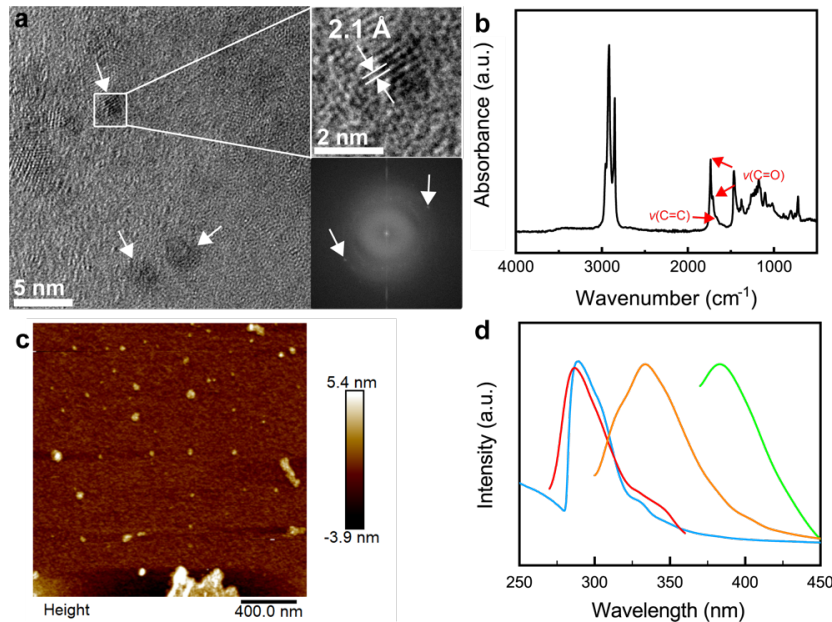

Figure 6.4 a) TEM image, b) single reflection ATR-FTIR absorbance spectrum and c)

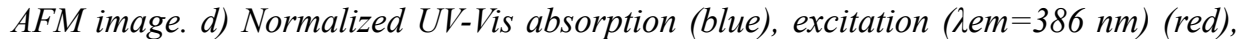
and emission spectra ( $(\lambda e m=286 \mathrm{~nm}$ (orange) and $340 \mathrm{~nm}$ (green)) of the isolated CQDs and the polymeric residue suspended in toluene.

The residual polymer and the fact that the samples for TEM imaging were retrieved as a yellowish paste indicate the presence of degraded polymer that remained in the toluene during liquid nitrogen quenching and was not removed by filtration and drying. Figure $6.4 \mathrm{~b}$ shows an FTIR spectrum of the yellowish paste obtained. The spectrum has all the representative peaks of $\mathrm{PE}$ with additional peaks located at $1712 \mathrm{~cm}^{-1}$ and 1736 $\mathrm{cm}^{-1}$ corresponding to absorbances of $\mathrm{C}=\mathrm{O}$ from carboxylic acids and aldehyde, respectively. ${ }^{52}$ Those peaks typically indicate thermooxidative degradation of $\mathrm{PE} .{ }^{53}$ There is also a noticeable small peak located at $1660 \mathrm{~cm}^{-1}$, indicating that $\mathrm{C}=\mathrm{C}$ bonds were also formed. ${ }^{54}$ We note, that as described in the literature, oxidation of $\mathrm{PE}$ is required for the formation of CQDs. ${ }^{45}$ The sharp peak $(289 \mathrm{~nm})$ and the small shoulder (330-340 $\left.\mathrm{nm}\right)$ from the Ultraviolet-Visible (UV-Vis) spectrum of the CQD suspension (Figure 6.4d) are ascribed to the $\pi-\pi^{*}$ transition of aromatic ring structures $(C=C)$ and the $n-\pi^{*}$ transition of oxidized groups $(\mathrm{C}=\mathrm{O})$, respectively. ${ }^{55,56}$ The presence of $\mathrm{C}=\mathrm{C}\left(\pi-\pi^{*}\right.$ transition) and $\mathrm{C}=\mathrm{O}\left(\mathrm{n}-\pi^{*}\right.$ transition) absorbance in the UV-Vis spectrum are in agreement with the structures observed in the FTIR spectrum, shown in Figure 6.4b. The normalized excitation spectrum exhibits a peak and shoulder combination that is similar to the UV-Vis spectrum. The excitation at $286 \mathrm{~nm}$ and $330-340 \mathrm{~nm}$ correspond to the CQD core (aromatic ring structure) and surface defects $(\mathrm{C}=\mathrm{O}$ containing groups $) .{ }^{57}$ The emission bands located at $337 \mathrm{~nm}$ and $383 \mathrm{~nm}$ are ascribed to the surface defects of CQDs. It is worth noting that when excited at $286 \mathrm{~nm}$, the emission spectrum shows a small shoulder around $320 \mathrm{~nm}$. This small shoulder is attributed to the photon emitted 
directly from the CQD cores without being trapped by surface defects. ${ }^{58}$

The absence of fluorescent and degradation products of PE heated without dispersed $\mathrm{SiO}_{2}$ NPs confirms that the $\mathrm{SiO}_{2}$ NPs play a role in forming the fluorescent CQDs. Therefore, to better understand the mechanism behind CQDs' formation during thermal treatment, one must first understand the role that the $\mathrm{SiO}_{2} \mathrm{NPs}$ play in the reaction, which we elucidate in the following section.

\subsubsection{Role of $\mathrm{SiO}_{2} \mathrm{NPs}$ in CQD Formation in Heated $\mathrm{PE} / \mathrm{SiO}_{2}$ Solutions}

Dynamic light scattering (DLS) was used to investigate the dispersion of the $\mathrm{SiO}_{2}$ NPs in toluene. Additionally, the morphology of $\mathrm{SiO}_{2}$ was observed by SEM in the PE matrix at room temperature. For DLS, the hydrodynamic radius of $\mathrm{SiO}_{2} \mathrm{NPs}$ in water was also determined. Figure $6.5 \mathrm{a}$ and Figure $6.5 \mathrm{~b}$ show the hydrodynamic particle size of $\mathrm{SiO}_{2}$ NPs suspended in water and in toluene, respectively.
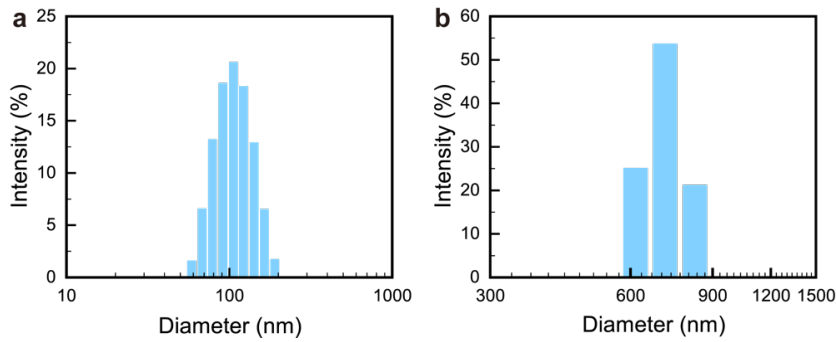

Figure 6.5 The hydrodynamic particle size of $\mathrm{SiO}_{2} \mathrm{NPS}$ dispersed in a) water and b) toluene.

The range of hydrodynamic particle diameter values of the $\mathrm{SiO}_{2} \mathrm{NPs}$ in water is around $110 \pm 3 \mathrm{~nm}$, which agrees with the determined dry particle size by SEM (Figure $6.6 b$ ). This confirms that after drying, the $\mathrm{SiO}_{2} \mathrm{NPs}$ did not irreversibly aggregate. The hydrodynamic particle diameter values of the $\mathrm{SiO}_{2} \mathrm{NPs}$ in toluene, however, varied in the range of $750 \pm 45 \mathrm{~nm}$, which is nearly seven times larger than the primary particle size, pointing at severe particle agglomeration in toluene. Figure 6.6 shows SEM images of the cross-sections of $1-, 2.5-$ and $4-\mathrm{PE} / \mathrm{SiO}_{2}$ films prepared after only 10 minutes of reaction time from the respective $\mathrm{SiO}_{2} \mathrm{PE}$ solutions. Figure 6.6 shows that almost all $\mathrm{SiO}_{2}$ NPs are present as part of large agglomerates (with sizes exceeding $1 \mu \mathrm{m}$ ) in 1- and 2.5$\mathrm{PE} / \mathrm{SiO}_{2}$ films, while in 4-PE/SiO 2 thin film, the observed $\mathrm{SiO}_{2} \mathrm{NPs}$ formed a large number of smaller agglomerates with a diameter in the sub-micrometer range. Based on these results, we conclude that the $\mathrm{SiO}_{2} \mathrm{NPs}$ agglomerated during the thermal treatment process. Like in mesoporous $\mathrm{SiO}_{2}$ particles (aerogels), the silica agglomerates in the $\mathrm{PE} /$ toluene mixture have an open porous structure. According to the literature, these pores 
may act as nanometric size reactors that prolong the lifetime of radicals produced from the thermal degradation of polyolefins. ${ }^{59}$ These long-lived radicals confined in the pores have more chance to react with other PE molecules, accelerating the thermal degradation of PE. The confinement effect on the kinetics of enzymatic reactions has been shown earlier by us and presumably can be extended to the radical activities of degraded PE. ${ }^{60}$ Typically, the thermal degradation rate for PE is low at $110{ }^{\circ} \mathrm{C} .{ }^{61}$ When these nanometricsized reactors are present, we increased the $\mathrm{PE}$ degradation reaction rate at lower temperatures and prepared CQDs from the degradation products within few hours.
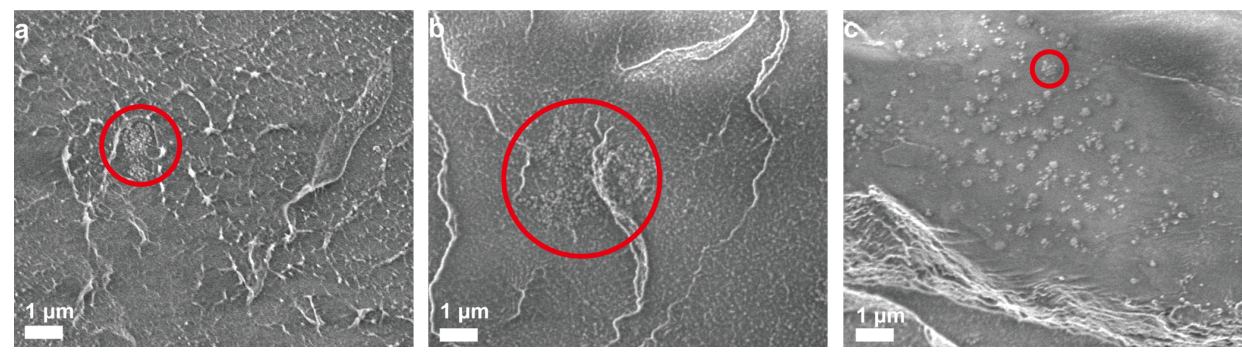

Figure 6.6 SEM images of cross-sectioned a) 1-PE/SiO 2 , b) 2.5-PE/SiO 2 , and c) 4$\mathrm{PE} / \mathrm{SiO}_{2}$ thin films after 10 minutes of thermal treatment. The red circles indicate $\mathrm{SiO}_{2}$ NP agglomerates.

Heat is a known physical factor that triggers the formation of macroradicals when accompanied by oxygen ${ }^{62}$ :

$$
\mathrm{RH}+\mathrm{O}_{2} \rightarrow\left[\mathrm{RHO}_{2}\right] \stackrel{\Delta}{\rightarrow} \mathrm{R}^{*}+\mathrm{HO}_{2}^{*}
$$

$\mathrm{RH}$ represents the monomer units of $\mathrm{PE}\left(\mathrm{CH}_{2}\right)$. Typically, the free radicals in solution at elevated temperature have a very short half-life time, in the range of $10^{-9}$ to $10^{-6} \mathrm{~s}$. However, the pores/cavities accessible from the surface of $\mathrm{SiO}_{2} \mathrm{NP}$ agglomerates can act as nanometer-sized reaction vessels that prolong the half-life of free radicals and therefore accelerate the degradation of $\mathrm{PE} .{ }^{59}$ Consequently, the $\mathrm{HO}_{2} *$ radicals formed in the nanocavities remain active for a longer time and have a higher chance of reacting with $\mathrm{RH}$ units to produce more $\mathrm{R}^{*}$ radicals. Subsequently, the free radicals $\mathrm{R}^{*}$ react with adjacent $\mathrm{RH}$ units to form vinyl groups resulting in chain-scission and the formation of $\mathrm{C}=\mathrm{C}$ bonds:

$$
\sim \mathrm{C}^{*} \mathrm{H}-\mathrm{CH}_{2}-\mathrm{CH}_{2} \sim \rightarrow \sim \mathrm{CH}=\mathrm{CH}_{2}+\mathrm{C}^{*} \mathrm{H}_{2} \sim
$$

This mechanism is supported by the appearance of the observed $\mathrm{C}=\mathrm{C}$ absorbance band at $1660 \mathrm{~cm}^{-1}$ in the FTIR spectrum (Figure 6.4b), indicating the presence of vinyl groups in CQDs and in the polymer residue. ${ }^{63}$ During CQD formation, the initially 
degraded polymer forms $\mathrm{C}=\mathrm{C} / \mathrm{C}-\mathrm{C}$ frameworks inside the porous agglomerated $\mathrm{SiO}_{2} \mathrm{NPs}$ during the thermal degradation/oxidation, thereby acting as skeletons/nucleus. As degradation progresses, these nuclei grow to nanometer-sized CQDs. ${ }^{64}$

Free radicals $\mathrm{R}^{*}$ can also undergo oxidation with oxygen to form peroxide radicals $\left(\mathrm{RO}_{2}{ }^{*}\right)$, followed by reacting with neighboring monomer units to form hydroperoxides $(\mathrm{ROOH})^{62}$ :

$$
\begin{gathered}
\mathrm{R}^{*}+\mathrm{O}_{2} \rightarrow \mathrm{RO}_{2}{ }^{*} \\
\mathrm{RO}_{2}{ }^{*}+\mathrm{RH} \rightarrow \mathrm{ROOH}+\mathrm{R}^{*} \rightarrow \mathrm{ROH}+\mathrm{RO}^{*}
\end{gathered}
$$

The presence of carboxylic acid $(\mathrm{ROOH})$ and aldehyde $(\mathrm{ROH})$ moieties formed by the thermal oxidation are also supported by the observed sharp peaks $(\mathrm{C}=\mathrm{O})$ located at 1712 and $1736 \mathrm{~cm}^{-1}$ in the FTIR spectrum (Figure 6.3), respectively. The formation of $\mathrm{C}=\mathrm{O}$ can occur either on the CQDs resulting in the presence of impurities and surface defects in the CQDs, or on the polymer chains resulting in the formation of carbonyl groups. As it was not among the objectives of the scope of this study, we cannot confirm whether the CQDs do or do not have these defects. This requires more extensive purification and characterization of the CQDs.

\subsubsection{Effect of $\mathrm{SiO}_{2}$ NPs Concentration of Fluorescent Intensity of $\mathrm{PE} / \mathrm{SiO}_{2}$ Thin Films}

To further investigate the influence of $\mathrm{SiO}_{2}$ NPs on the fluorescent properties, $\mathrm{PE} / \mathrm{SiO}_{2}$ composites with particle concentrations of $1,2.5$, and $4 \mathrm{wt} \%$ were prepared with up to 24 hours of thermal treatment at $110{ }^{\circ} \mathrm{C}$ in air. The as-prepared composites were proceeded into thin films and measured with a fluorescent microscope. Figure 6.7 shows the grey values of the fluorescence of 1-, 2.5-, and 4- $\mathrm{PE} / \mathrm{SiO}_{2}$ against reaction time. We note that the $\mathrm{PE} / \mathrm{SiO}_{2} /$ toluene mixture needed the first 10 minutes to homogenize due to its high viscosity. As a consequence, 10 minutes is the first experimentally available reaction time for taking aliquots for further study. Furthermore, we note, that the differences in baseline grey value intensities of the PE films with different $\mathrm{SiO}_{2} \mathrm{NP}$ loading (resulting from the differences in particle loading) lead to changes in the refraction of the excitation/emission light beams.

Interestingly, there is no significant change in the measured fluorescent intensity during the first few hours of the reaction for 1- and 2.5-PE/ $\mathrm{SiO}_{2}$. However, the fluorescent intensity gradually increases for the 4-PE/SiO 2 film right after the start of the reaction. In fact, for 1-PE/ $/ \mathrm{SiO}_{2}$ films, even after 24 hours of thermal treatment, there is no noticeable 
change in the fluorescence intensity. However, the fluorescent intensity increased significantly for $2.5-\mathrm{PE} / \mathrm{SiO}_{2}$ films after 8 hours of thermal treatment.

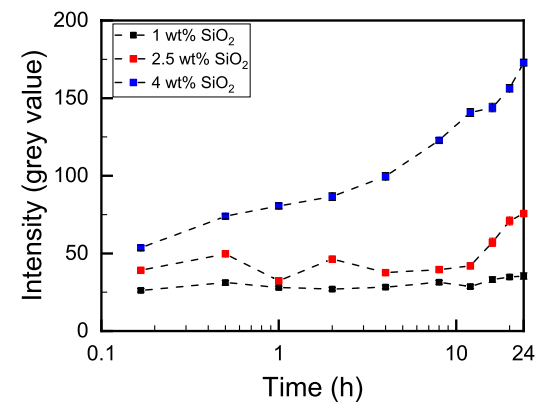

Figure 6.7 Grey value intensity of as-prepared 1-PE/SiO $2,2.5-\mathrm{PE} / \mathrm{SiO}_{2}$, and 4-PE/SiO${ }_{2}$ films as a function of the reaction time. The reaction was performed at $110^{\circ} \mathrm{C}$ in air.

This remarkable difference is explained by the relatively high concentration of $\mathrm{SiO}_{2}$ agglomerates present in 4-PE/SiO 2 (Figure 6.6c). The increased amount of smaller $\mathrm{SiO}_{2}$ agglomerates results in an increased specific contact area between the $\mathrm{SiO}_{2} \mathrm{NPs}$ and PE chains. Consequently, the relative number of accessible nanocavities to accelerate the CQD formation increases. For 1- and 2.5- $\mathrm{PE} / \mathrm{SiO}_{2}$, the relatively low particle surface area available for promoting the CQD formation results in a reduced rate of CQD formation. The observed dependence of CQD formation on the concentration and size of $\mathrm{SiO}_{2}$ agglomerates confirms that the $\mathrm{SiO}_{2}$ agglomerates play a crucial role in forming CQDs at relatively low temperatures and that their exploitation as an enabling platform in the facile synthesis of CQD based FM is of interest for further exploration. For spectral engineering, we eventually need to be able to tune the emission wavelengths of the CQDs by varying the size of the emitters. The following sections show the first attempts to control the emission wavelengths of the CQDs and a proof of concept showing their potential as FMs in polymers to aid in sorting.

\subsubsection{Fluorescent Spectra of $\mathrm{PE} / \mathrm{SiO}_{2}$ Thin Films and the Influence of the Reaction Temperature}

In a first attempt to show the potential utilization of the CQDs as FMs, we examined their emission spectra as a function of particle concentration and reaction time upon excitation with $360 \mathrm{~nm}$ and $380 \mathrm{~nm}$ light, respectively. Figure $6.8 \mathrm{a}$ and $6.8 \mathrm{~b}$ show the fluorescent emission spectra of 2.5- and $4-\mathrm{PE} / \mathrm{SiO}_{2}$ thin films with an excitation wavelength of $360 \mathrm{~nm}$ and $380 \mathrm{~nm}$, respectively. 

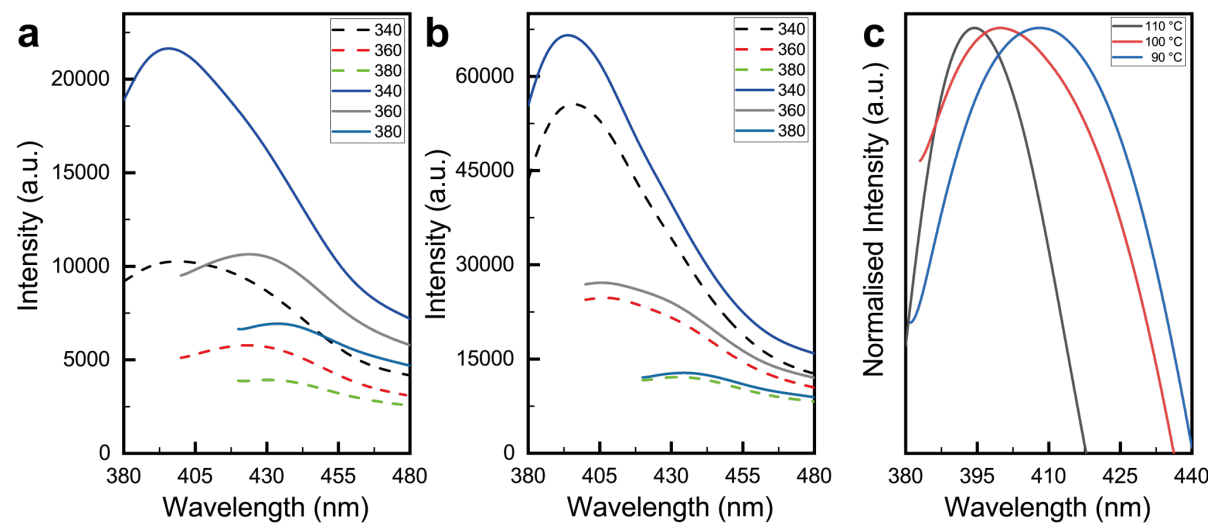

Figure 6.8 Fluorescent emission spectra of a) 2.5-PE/SiO${ }_{2}$ and b) 4-PE/SiO 2 thin films prepared in air with 20 hours (dashed lines) and 24 hours (solid lines) reaction times upon excitation with $340 \mathrm{~nm}, 360 \mathrm{~nm}$, and $380 \mathrm{~nm}$ wavelengths. c) Fluorescent emission spectra of thin films of 4-PE/SiO${ }_{2}$ upon excitation with $340 \mathrm{~nm}$ light, fabricated at reaction temperatures of $90^{\circ} \mathrm{C}, 100^{\circ} \mathrm{C}$, and $110^{\circ} \mathrm{C}$ for 24 hours in air.

The observed fluorescent emission maxima for the $\mathrm{PE} / \mathrm{SiO}_{2}$ samples fall into the blue emission range $(380 \mathrm{~nm}$ to $500 \mathrm{~nm}$ ), which is typically the result of the radiative recombination of e-h pairs in the $\mathrm{sp}^{2}$ cluster and surface defects of CQDs. ${ }^{65}$ A clear redshift of a few $\mathrm{nm}$ in the fluorescent emission is observed in the fluorescent spectra by increasing the excitation wavelength from 340 to $380 \mathrm{~nm}$. Although the exact mechanism for CQDs fluorescent emission is still under debate, it is generally agreed that the CQDs' size and surface status significantly influence the fluorescent properties of CQDs. ${ }^{66} \mathrm{We}$ consider that the excitation-dependent fluorescence behavior of our thin films is a combination of CQDs size dispersity, impurities, and surface defects. ${ }^{67}$ Furthermore, a modest redshift was also observed in the fluorescent emission spectrum when the reaction time increased from 20 to $24 \mathrm{~h}$. The observed redshift can be tentatively explained by the longer time available for the CQDs to grow, resulting in larger CQDs that typically have a redshift in their emission spectrum. ${ }^{43}$

Figure $6.8 \mathrm{c}$ shows normalized fluorescent emission spectra of $4-\mathrm{PE} / \mathrm{SiO}_{2}$ samples fabricated at different temperatures. One can observe in Figure $6.8 \mathrm{c}$ that upon increasing the reaction temperature from $90{ }^{\circ} \mathrm{C}$ to $110^{\circ} \mathrm{C}$ for the 4-PE/SiO 2 mixture, the emission peak shifts from $408 \mathrm{~nm}$ to $394 \mathrm{~nm}$ upon excitation with $340 \mathrm{~nm}$ light. It is known that upon increasing the reaction temperature, the oxygen solubility decreases. ${ }^{68}$ Therefore, it is reasonable to assume that upon increasing the reaction temperature from $90{ }^{\circ} \mathrm{C}$ to 110 ${ }^{\circ} \mathrm{C}$, fewer oxygen molecules were present in the reaction mixture, which reduced the chance for free radicals to be involved in oxidation reactions and the formation of $\mathrm{C}=\mathrm{O}$ 
species. This could decrease the number of surface traps and impurities $(\mathrm{C}=\mathrm{O})$ present in the formed CQDs leading to less energy loss for the fluorescent emission, resulting in higher emission energy for the CQDs prepared at higher temperatures. ${ }^{69}$

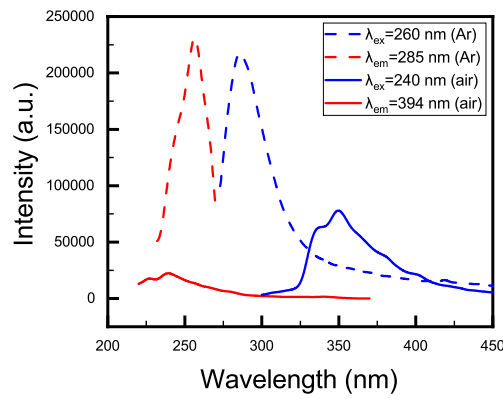

Figure 6.9 Fluorescent excitation and emission spectra of 4-PE/SiO $\mathrm{S}_{2}$ thin films prepared with 24 h reaction time in air (solid line) and Ar (dashed line) atmosphere.

To further elucidate the role oxygen during the CQD synthesis, the reaction was performed in $\mathrm{Ar}$ atmosphere for 4-PE/ $\mathrm{SiO}_{2}$ mixtures at $110{ }^{\circ} \mathrm{C}$. The corresponding fluorescent excitation and emission spectra of the 4-PE/ $\mathrm{SiO}_{2}$ samples are shown in Figure 6.9. The maximum excitations for 4-PE/ $/ \mathrm{SiO}_{2}$ synthesized in air and $\mathrm{Ar}$ are located at 240 $\mathrm{nm}$ and $260 \mathrm{~nm}$, respectively. These excitation peaks are ascribed to the photon absorption of $\mathrm{C}=\mathrm{C}$ (aromatic structure) in the CQD core. ${ }^{70}$ The corresponding emission centers are located at $350 \mathrm{~nm}$ and $285 \mathrm{~nm} .{ }^{57}$ The blueshift in emission wavelength upon substituting air with Ar is ascribed to the reduced presence of surface defects (surface oxidation) of the CQDs when prepared without the presence of oxygen. This means that the photons are directly emitted from the aromatic ring structure $(\mathrm{C}=\mathrm{C})$ upon excitation and that without the presence of surface defects to trap these photons, they can maintain a higher energy level, therefore, emitting at a shorter wavelength. ${ }^{58}$

Overall, these results demonstrate that with minor modifications to the reaction conditions, changes in the CQD emissions are accessible, rendering our system a valuable designer platform for the controlled synthesis of PE-based FMs at mild reaction conditions.

\subsubsection{Solution Blending CQDs and $\mathrm{PE} / \mathrm{SiO}_{2}$ Composites with Pure $\mathrm{PE}$}

We used solution blending to prepare CQD containing PE films to demonstrate the applicability of the CQD as fluorescent markers in virgin polymers. As-prepared CQD 4$\mathrm{PE} / \mathrm{SiO}_{2}$ (114 hours reaction at $110{ }^{\circ} \mathrm{C}$ in air) and isolated CQDs (extracted from 4$\mathrm{PE} / \mathrm{SiO}_{2}$ after 24 hours reaction in air) were used as tracer batches to prepare PE films 
with a tracer loading of $10 \mathrm{wt} \%$ (meaning the actual CQD concentration is unknown). Following compression molding, film samples were cut to easily recognizable shapes, i.e. circles and triangles, respectively. In addition, pristine PE films were prepared and cut to rectangular shapes for comparison. Five pieces were placed together from each PE film type, and photos were taken in daylight (Figure 6.10a) and upon excitation with 360 nm light (Figure 6.10b).
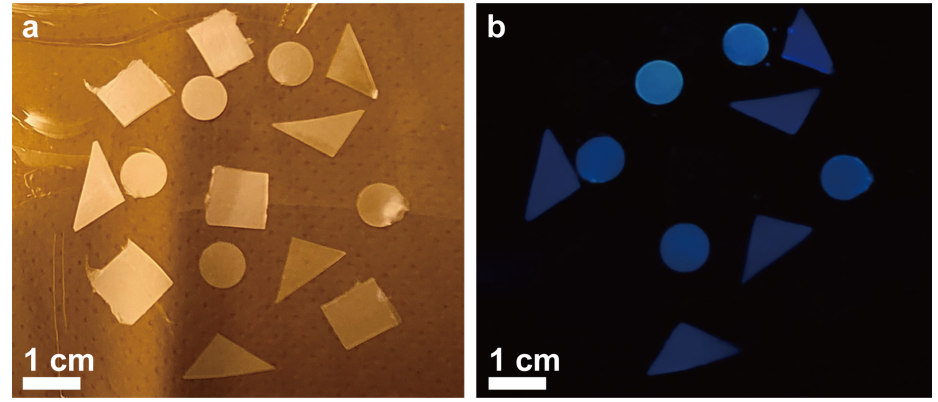

Figure 6.10 Photos of pure PE (rectangle), PE/CQD (triangle), and CQD 4-PE/SiO (circular) films in a) daylight and b) upon excitation with $367 \mathrm{~nm}$ light.

In Figure 6.10a (photo taken in daylight), all pieces are visible and their shape is clearly identifiable. Upon excitation with $367 \mathrm{~nm}$ light, only the CQD-loaded pieces are visible by their emission in the blue range. The color difference (not intensity) between triangular (darker blue) and circular (lighter blue) pieces under $367 \mathrm{~nm}$ excitation is due to differences in thermal treatment time, resulting in the presence of larger CQDs in the circular PE films, as discussed earlier. The observed modest redshift is in agreement with this observation. ${ }^{43}$ Also, from this simple visual experiment, we learn that the embedded $\mathrm{SiO}_{2}$ NPs do not affect the PE samples' detectability. Overall, we conclude that the simple preparation method at mild reaction conditions of CQD FMs in $\mathrm{SiO}_{2} / \mathrm{PE}$ mixtures described here is a promising approach for fluorescence marking. Further in-depth exploration to enhance the CQD traceability is needed and is underway to explain details of the fluorescence. In addition, upon expanding the variations and control of emission wavelengths, our simple preparation method without requiring extensive purification methods opens a broad range of novel applications, e.g., counter faking, sorting, tracing, etc.

\subsection{Conclusion}

PE, containing CQDs markers with tunable fluorescence was prepared by a facile thermal approach operated at mild conditions. For the reaction employed, solvent- 
induced $\mathrm{SiO}_{2} \mathrm{NP}$ aggregation provided surface-exposed nanocavities that were assumed to function as nanoreactors, enabling CQDs to form at relatively low reaction temperatures. TEM imaging of isolated CQD particles, UV spectroscopy, and fluorescent measurements confirmed the successful preparation of CQDs. The emission maximum for PE/CQD samples was tunable between $394 \mathrm{~nm}$ to $408 \mathrm{~nm}$ by decreasing the reaction temperature from $110{ }^{\circ} \mathrm{C}$ to $90{ }^{\circ} \mathrm{C}$. PE/CQD samples prepared in the absence of oxygen only emitted in the ultraviolet region $(286 \mathrm{~nm})$. Finally, we successfully exploited CQDs with a different emission maximum as FMs in PE compression molded films. Upon irradiating with $367 \mathrm{~nm}$ light, the different PE samples were readily visually identified.

\subsection{Materials and Methods}

Materials. Tetraethyl orthosilicate (TEOS) $\geq 99.0 \%$, toluene 99.5\%, and 2-propanol 99.5\% were purchased from Aldrich (Milwaukee, WI, USA). Ammonium hydroxide solution 28-30\%, triethylamine (TEA) 99.5\%, and PE (medium density, MQ 200) were purchased from Sigma-Aldrich (St. Louis, MO, USA). Ethanol (EtOH) absolute for analysis was purchased from Merck (Darmstadt, Germany). Milli-Q water was produced by a Millipore Synergy system (Billerica, MA, USA). Unless otherwise mentioned, all chemicals were used as received.

Stöber Synthesis of Silica Nanoparticles. $\mathrm{SiO}_{2} \mathrm{NPs}$ with a diameter of $\sim 110 \mathrm{~nm}$ were prepared by the Stöber method. ${ }^{46}$ In a typical reaction, $168 \mathrm{ml} \mathrm{EtOH}$ was mixed with $28 \mathrm{ml}$ Milli-Q water and $30 \mathrm{ml}$ TEOS in the presence of $2 \mathrm{ml}$ ammonium hydroxide in a round-bottom flask by stirring for 1.5 hours at $500 \mathrm{rpm}$ at room temperature. Subsequently, the $\mathrm{SiO}_{2}$ NP dispersion was centrifuged at 10,000 rpm for $30 \mathrm{~min}$. Then, the collected $\mathrm{SiO}_{2}$ NPs were redispersed in 2-propanol and centrifuged again. This washing step was repeated twice, followed by vacuum drying of the purified and collected $\mathrm{SiO}_{2}$ NPs at room temperature for 12 hours.

Silica Nanoparticle Characterization. A high-resolution scanning electron microscope (JEOL Field Emission JSM-7610F Plus, JEOL Benelux, Nieuw-Vennep, the Netherlands) was used to study the morphology of the $\mathrm{SiO}_{2} \mathrm{NPs}$ particles. The electron acceleration voltage was typically between 3 to $5 \mathrm{keV}$. Fourier Transform Infrared (FTIR) spectra were collected by a Bruker ALPHA single attenuated total reflection (ATR) FTIR spectrometer equipped with an ATR single reflection crystal (Bruker Optic GmbH, Ettlingen, Germany). The spectra were collected in the range of $400-4000 \mathrm{~cm}^{-1}$ (spectral resolution of $4 \mathrm{~cm}^{-1}, 1280$ scans). Background spectra were recorded against air. The asprepared $\mathrm{SiO}_{2} \mathrm{NPs}$ were dispersed in water and toluene at a concentration of $2.5 \mathrm{wt} \%$ via 
intense sonication over $30 \mathrm{~min}$. The hydrodynamic radius values of the particles in water and toluene were measured by Dynamic Light Scattering (DLS) (Malvern Zetasizer Nano-ZS, Malvern Instruments, Malvern, UK).

Synthesis of Fluorescent Polyethylene Silica Nanocomposites in Air. We used two pathways to obtain FM containing PE, i.e., in air and under Ar atmosphere. First, we describe the preparative steps in air. We started with $0.4 \mathrm{~g} 110 \mathrm{~nm} \mathrm{SiO} 2 \mathrm{NPs}$, which were suspended in $10 \mathrm{ml}$ toluene in a round-bottom flask and exposed to $30 \mathrm{~min}$ intense sonication, followed by heating the suspension formed under vigorous stirring to $110{ }^{\circ} \mathrm{C}$ by immersing the flask in a thermostated oil bath. Simultaneously, 9.6 g PE was dissolved in $30 \mathrm{ml}$ toluene employing vigorous stirring in a round-bottom flask, which was immersed in a thermostated oil bath at $90{ }^{\circ} \mathrm{C}$. Subsequently, the PE/toluene mixture was transferred to a reflux setup, followed by adding the $\mathrm{SiO}_{2} \mathrm{NPs}$ to the toluene suspension. After adding the $\mathrm{SiO}_{2} \mathrm{NPs}$ suspension, the reaction mixture was refluxed at $110^{\circ} \mathrm{C}$ for 24 hours. Finally, the excess toluene was removed by drying the reaction mixture under a gentle nitrogen flow for 5 hours. The $\mathrm{PE} / \mathrm{SiO}_{2}$ was dried to constant weight by vacuum drying at $40{ }^{\circ} \mathrm{C}$ for 24 hours. This resulted in a $\mathrm{PE} / \mathrm{SiO}_{2}$ composite with a $\mathrm{SiO}_{2} \mathrm{NP}$ loading of $4 \mathrm{wt} \%$. This composite is abbreviated as $4-\mathrm{PE} / \mathrm{SiO}_{2}$. The same procedure was followed to produce $1.0 \mathrm{wt} \%$ and $2.5 \mathrm{wt} \% \mathrm{PE} / \mathrm{SiO}_{2}\left(1-\mathrm{PE} / \mathrm{SiO}_{2}\right.$ and $\left.2.5-\mathrm{PE} / \mathrm{SiO}_{2}\right)$ in which 0.1 and $0.25 \mathrm{~g} \mathrm{SiO}_{2} \mathrm{NPs}$ and 9.9 and $9.75 \mathrm{~g} \mathrm{PE}$, were used, respectively. The amount of toluene used was not changed. Additionally, 4-PE/ $/ \mathrm{SiO}_{2}$ composites were prepared at $90{ }^{\circ} \mathrm{C}$ and $100{ }^{\circ} \mathrm{C}$, as well. (Unless otherwise mentioned, we refer to $\mathrm{PE} / \mathrm{SiO}_{2}$ prepared at $110^{\circ} \mathrm{C}$ in the main text.) The pure PE sample was prepared by refluxing in toluene at $110^{\circ} \mathrm{C}$ for 24 hours and dried using same procedure mentioned before as the control group.

Fluorescent Polyethylene Silica Nanocomposite Synthesis Under Argon. In order to test the influence of air on the reaction and on the reaction products, for comparison, synthesis was also performed in Ar atmosphere. For this process $0.04 \mathrm{~g} 110 \mathrm{~nm} \mathrm{SiO} 2 \mathrm{NPs}$ and $0.96 \mathrm{~g}$ PE were dried in a vacuum oven at $40{ }^{\circ} \mathrm{C}$ for $48 \mathrm{~h}$, then the mixture was transferred into a $50 \mathrm{ml}$ Schlenk tube with a magnetic stirring bar under a nitrogen environment (in a glove box). $4 \mathrm{ml}$ anhydrous toluene was added to the Schlenk tube. The Schlenk tube was sealed with a Teflon cap before it was removed from the glovebox. Next, the Schlenk tube was connected to a Schlenk line and flushed with Ar for10 mins. Subsequently, the Schlenk tube was heated under vigorous stirring by a thermostated oil bath at $90{ }^{\circ} \mathrm{C}$. After the PE was fully dissolved in the toluene, the Schlenk tube was disconnected from the Schlenk line and removed from the oil bath, followed by $15 \mathrm{~min}$ sonication in $90{ }^{\circ} \mathrm{C}$ water. After sonication, the Schlenk tube was reconnected to the Schlenk line under Ar and immersed into a thermostated oil bath at $110{ }^{\circ} \mathrm{C}$. The reaction 
was allowed to proceed under Ar with vigorous stirring for 24 hours. Next, the reaction mixture was extracted from the Schlenk tube and cooled in an ice bath, followed by drying under a gentle nitrogen flow for 5 hours to remove the excess toluene. Finally, the $\mathrm{PE} / \mathrm{SiO}_{2}$ mixture was dried to constant weight by vacuum drying at $40^{\circ} \mathrm{C}$ for 24 hours.

Film Preparation. A hot press (Fontijne, the Netherlands) was used to press $200 \mu \mathrm{m}$ thick $\mathrm{PE} / \mathrm{SiO}_{2}$ films in a stainless-steel mold $(4 \times 3 \mathrm{~cm})$. The processing temperature, applied load, and press time were $110^{\circ} \mathrm{C}, 200 \mathrm{kN}$, and 5 minutes, respectively.

Thin Film Characterization. An inverted Olympus IX71 fluorescence microscope equipped with an IX2-RFAC reflector turret filter cube (Olympus, Germany) and a PerkinElmer LS 55 fluorescence spectrometer (PerkinElmer, Waltham, MA, USA) were used to study the optical properties of the $\mathrm{PE} / \mathrm{SiO}_{2}$ films. The filter cubes' excitation and emission cut-offs were $360-370 \mathrm{~nm}$ and $460 \mathrm{~nm}$, respectively. The emission spectra were recorded by a fluorescence spectrometer with an excitation wavelength of 340,360, and $380 \mathrm{~nm}$, respectively. In addition, Scanning Electron Microscopy (SEM) was performed by a JEOL Field Emission JSM-7610F Plus instrument (JEOL Benelux, Nieuw-Vennep, the Netherlands) to study the cross-section of the $\mathrm{PE} / \mathrm{SiO}_{2}$ thin films. Before analysis, the thin films were freeze-fractured after cooling in liquid nitrogen for $15 \mathrm{~min}$.

Carbon Quantum Dot Extraction. 1 gram of 4-PE/SiO 2 was dissolved in $10 \mathrm{ml}$ toluene thermostated at $90{ }^{\circ} \mathrm{C}$ in an oil bath. Following the complete dissolution of the 4$\mathrm{PE} / \mathrm{SiO}_{2}$, the mixture was quenched into liquid nitrogen and left to cool down to below ambient for 15 mins to avoid PE crystallization. Subsequently, the excess liquid nitrogen was removed, and the sample was left on the bench to heat up slowly to room temperature. Next, the supernatant was filtered with a $200 \mathrm{~nm}$ pore size glass filter (Merck Millipore, USA) to remove the suspended agglomerated $\mathrm{SiO}_{2} \mathrm{NPs}$, followed by collecting the CQDs by solvent removal of the filtrate via evaporation under a gentle nitrogen flow, which left a yellowish paste as residue for analysis.

Carbon Quantum Dot Characterization. Transmission Electron Microscopy (TEM) was performed by a FEI/Philips CM300 system (FEI, Eindhoven, the Netherlands). Diluted CQD dispersions in toluene were deposited on the carbon side of a carbon/copper grid (HC200-Cu) (EMS, Germany) before imaging. Images were obtained in the bright field mode at a $300 \mathrm{kV}$ acceleration voltage. Ultraviolet/Visible (UV-Vis) spectroscopy was performed by a LAMBDA $850 \mathrm{UV}-\mathrm{Vis}$ spectrometer (PerkinElmer, Waltham, MA, USA). The CQDs were dispersed in toluene via 10 mins intense sonication. The CQD suspension was measured against pure toluene. The excitation and emission spectra were recorded by a PerkinElmer LS 55 fluorescence 166 
spectrometer (PerkinElmer, Waltham, MA, USA) with an emission wavelength of 384 and excitation wavelengths of 286 and $340 \mathrm{~nm}$.

Solution Blending. $20 \mathrm{mg}$ of CQDs (extracted) was solution blended with $180 \mathrm{mg}$ $\mathrm{PE}$ in $5 \mathrm{ml}$ toluene under vigorous stirring in a round-bottom flask, while thermostated in an oil bath at $80{ }^{\circ} \mathrm{C}$. After $30 \mathrm{~min}$, the excess toluene was removed by drying the reaction mixture under a gentle nitrogen flow for 5 hours. The $\mathrm{PE} / \mathrm{SiO}_{2}$ composite was dried to constant weight by vacuum drying at $40{ }^{\circ} \mathrm{C}$ for 24 hours. The same procedure was used to blend $20 \mathrm{mg}$ of $114 \mathrm{~h}$ thermal treated $\mathrm{PE} / \mathrm{SiO}_{2}$ (non-extracted) with $180 \mathrm{mg}$ of PE. The as-prepared nanocomposites were pressed to films (see section Film Preparation) and cut to different shapes before being observed under daylight and a $360 \mathrm{~nm}$ light source to identify the respective PE samples.

\subsection{Supporting Information}

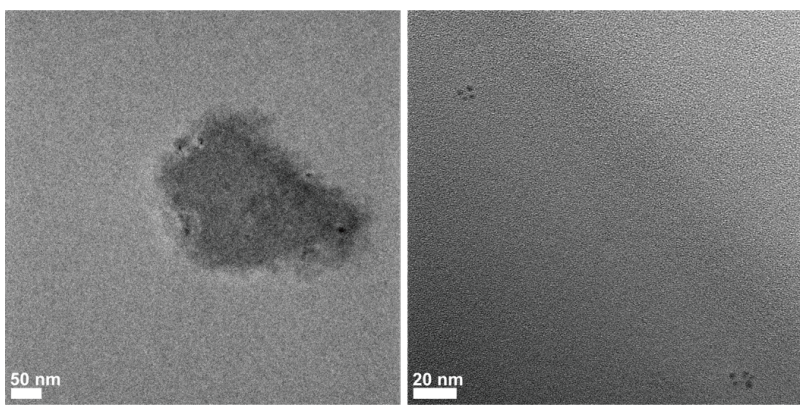

Figure S6.1 TEM image of as-prepared isolated CQDs embedded in PE polymeric residue after liquid nitrogen quenching and filtration.

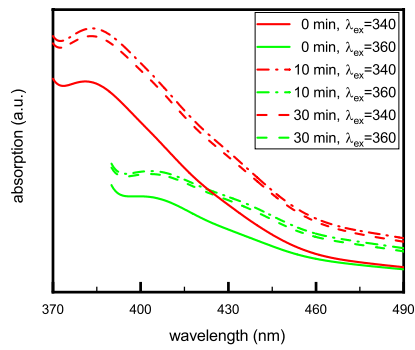

Figure S6.2 Emission spectra of isolated CQDs suspended in toluene after 0 min (solid line), $10 \mathrm{~min}$ (dash-dot line) and $30 \mathrm{~min}$ (dash line) sonication with $340 \mathrm{~nm}$ (red) and 360 nm (green) excitation. 


\subsection{References}

(1) Ruj, B.; Pandey, V.; Jash, P.; Srivastava, V. K. Sorting of Plastic Waste for Effective Recycling. Int. Journal of Applied Sciences and Engineering Research 2015, 4 (4), 564-571.

(2) Ragaert, K.; Delva, L.; van Geem, K. Mechanical and Chemical Recycling of Solid Plastic Waste. Waste Management 2017, 69, 24-58.

(3) Singh, N.; Hui, D.; Singh, R.; Ahuja, I. P. S.; Feo, L.; Fraternali, F. Recycling of Plastic Solid Waste: A State of Art Review and Future Applications. Composites Part B: Engineering 2017, 115, 409-422.

(4) Pol, V. G.; Thiyagarajan, P. Remediating Plastic Waste into Carbon Nanotubes. Journal of Environmental Monitoring 2010, 12 (2), 455-459.

(5) Müller, R. J.; Kleeberg, I.; Deckwer, W. D. Biodegradation of Polyesters Containing Aromatic Constituents. Journal of Biotechnology 2001, 86 (2), 87-95.

(6) Manfredi, S.; Tonini, D.; Christensen, T. H.; Scharff, H. Landfilling of Waste: Accounting of Greenhouse Gases and Global Warming Contributions. Waste Management and Research 2009, 27 (8), 825-836.

(7) Rhodes, C. J. Plastic Pollution and Potential Solutions. Science progress 2018, 101 (3), 207-260.

(8) Tall, S. (2000). Recycling of Mixed Plastic Waste - Is Separation Worthwhile?, Royal Institute of Technology. https://www.divaportal.org/smash/get/diva2:8665/FULLTEXT01.pdf

(9) Geyer, R.; Jambeck, J. R.; Law, K. L. Production, Use, and Fate of All Plastics Ever Made. Science Advances 2017, 3 (7), 10.1126/sciadv.1700782.

(10) Verma, R.; Vinoda, K. S.; Papireddy, M.; Gowda, A. N. S. Toxic Pollutants from Plastic Waste- A Review. Procedia Environmental Sciences 2016, 35, 701-708.

(11) Johnke, B. Emissions From Waste Incineration. Intergovernmental Panel on Climate Change 1998, 455-468.

(12) Hopewell, J.; Dvorak, R.; Kosior, E. Plastics Recycling: Challenges and Opportunities. Philosophical Transactions of the Royal Society B: Biological Sciences 2009, 364 (1526), 2115-2126.

(13) Ross, S.; Evans, D. The Environmental Effect of Reusing and Recycling a PlasticBased Packaging System. Journal of Cleaner Production 2003, 11 (5), 561-571.

(14) Taranic, I.; Behrens, A.; Corrado, T. Understanding the Ciruclar Economy in Europe from Resource Efficiency to Sharing Platforms: The CEPS Framework. CEPS Special Reports 2016, 143, 29.

(15) Wang, C. qing; Wang, H.; Fu, J. gang; Liu, Y. nian. Flotation Separation of Waste Plastics for Recycling-A Review. Waste Management 2015, 41, 28-38.

(16) Reinsch, E.; Frey, A.; Albrecht, V.; Simon, F.; Peuker, U. Continuous Electric Sorting in the Recycling Process of Plastics. Chemie Ingenieur Technik 2014, 86, 784-796.

(17) Chanda, M.; Roy, S. K. Plastics Technology Handbook; CRC Press, 2006.

(18) Censori, M.; La Marca, F.; Carvalho, M. T. Separation of Plastics: The Importance of Kinetics Knowledge in the Evaluation of Froth Flotation. Waste Management 2016, 54, 39-43.

(19) Thiounn, T.; Smith, R. C. Advances and Approaches for Chemical Recycling of 
Plastic Waste. Journal of Polymer Science 2020, 58 (10), 1347-1364.

(20) Al-Salem, S. M.; Lettieri, P.; Baeyens, J. Recycling and Recovery Routes of Plastic Solid Waste (PSW): A Review. Waste Management 2009, 29 (10), 26252643.

(21) Brunner, S.; Fomin, P.; Kargel, C. Automated Sorting of Polymer Flakes: Fluorescence Labeling and Development of a Measurement System Prototype. Waste Management 2015, 38 (1), 49-60.

(22) Maris, E.; Aoussat, A.; Naffrechoux, E.; Froelich, D. Polymer Tracer Detection Systems with UV Fluorescence Spectrometry to Improve Product Recyclability. Minerals Engineering 2012, 29, 77-88.

(23) Massardier, V.; Louizi, M.; Maris, E.; Froelich, D. High Shear Dispersion of Tracers in Polyolefins for Improving Their Detection. Polimeros 2015, 25 (5), 466-476.

(24) Ahmad, S. R. A New Technology for Automatic Identification and Sorting of Plastics for Recycling. Environmental Technology 2004, 25 (10), 1143-1149.

(25) Langhals, H.; Zgela, D.; Schlücker, T. High Performance Recycling of Polymers by Means of Their Fluorescence Lifetimes*. Green and Sustainable Chemistry 2014, 04 (03), 144-150.

(26) Gao, G.; Turshatov, A.; Howard, I. A.; Busko, D.; Joseph, R.; Hudry, D.; Richards, B. S. Up-Conversion Fluorescent Labels for Plastic Recycling: A Review. Advanced Sustainable Systems 2017, 1 (5), 160003.

(27) Arenas-Vivo, A.; Beltrán, F. R.; Alcázar, V.; de la Orden, M. U.; Martinez Urreaga, J. Fluorescence Labeling of High Density Polyethylene for Identification and Separation of Selected Containers in Plastics Waste Streams. Comparison of Thermal and Photochemical Stability of Different Fluorescent Tracers. Materials Today Communications 2017, 12 (July), 125-132.

(28) Woidasky, J.; Sander, I.; Schau, A.; Moesslein, J.; Wendler, P.; Wacker, D.; Gao, G.; Kirchenbauer, D.; Kumar, V.; Busko, D.; Howard, I. A.; Richards, B. S.; Turshatov, A.; Wiethoff, S.; Lang-Koetz, C. Inorganic Fluorescent Marker Materials for Identification of Post-Consumer Plastic Packaging. Resources, Conservation and Recycling 2020, 161, 104976.

(29) Field, M. S.; Wilhelm, R. G.; Quinlan, J. F.; Aley, T. J. An Assessment of the Potential Adverse Properties of Fluorescent Tracer Dyes Used for Groundwater Tracing. Environmental Monitoring and Assessment 1995, 38 (1), 75-96.

(30) Tchounwou, P. B.; Yedjou, C. G.; Patlolla, A. K.; Sutton, D. J. Heavy Metal Toxicity and the Environment. Exs 2012, 101, 133-164.

(31) Ali, H.; Khan, E.; Ilahi, I. Environmental Chemistry and Ecotoxicology of Hazardous Heavy Metals: Environmental Persistence, Toxicity, and Bioaccumulation. Journal of Chemistry 2019, 2019, 6730305.

(32) Choyke, P. L.; Alford, R.; Simpson, H. M.; Duberman, J.; Craig Hill, G.; Ogawa, M.; Regino, C.; Kobayashi, H. Toxicity of Organic Fluorophores Used in Molecular Imaging: Literature Review. Molecular Imaging 2009, 8 (6), 341-354.

(33) Molaei, M. J. Principles, Mechanisms, and Application of Carbon Quantum Dots in Sensors: A Review. Analytical Methods 2020, 12 (10), 1266-1287.

(34) Zhang, Z.; Zhang, J.; Chen, N.; Qu, L. Graphene Quantum Dots: An Emerging Material for Energy-Related Applications and Beyond. Energy and 
Environmental Science 2012, 5 (10), 8869-8890.

(35) Shen, J.; Zhu, Y.; Yang, X.; Li, C. Graphene Quantum Dots: Emergent Nanolights for Bioimaging, Sensors, Catalysis and Photovoltaic Devices. Chemical Communications 2012, 48 (31), 3686-3699.

(36) Zheng, X. T.; Ananthanarayanan, A.; Luo, K. Q.; Chen, P. Glowing Graphene Quantum Dots and Carbon Dots: Properties, Syntheses, and Biological Applications. Small 2015, 11 (14), 1620-1636.

(37) Zhou, J.; Booker, C.; Li, R.; Zhou, X.; Sham, T. K.; Sun, X.; Ding, Z. An Electrochemical Avenue to Blue Luminescent Nanocrystals from Multiwalled Carbon Nanotubes (MWCNTs). Journal of the American Chemical Society 2007, 129 (4), 744-745.

(38) Sun, Y. P.; Zhou, B.; Lin, Y.; Wang, W.; Fernando, K. A. S.; Pathak, P.; Meziani, M. J.; Harruff, B. A.; Wang, X.; Wang, H.; Luo, P. G.; Yang, H.; Kose, M. E.; Chen, B.; Veca, L. M.; Xie, S. Y. Quantum-Sized Carbon Dots for Bright and Colorful Photoluminescence. Journal of the American Chemical Society 2006, 128 (24), 7756-7757.

(39) Huang, H.; Xu, Y.; Tang, C. J.; Chen, J. R.; Wang, A. J.; Feng, J. J. Facile and Green Synthesis of Photoluminescent Carbon Nanoparticles for Cellular Imaging. New Journal of Chemistry 2014, 38 (2), 784-789.

(40) Sharma, A.; Das, J. Small Molecules Derived Carbon Dots: Synthesis and Applications in Sensing, Catalysis, Imaging, and Biomedicine. Journal of Nanobiotechnology 2019, 17 (1), 92.

(41) Hu, Y.; Gao, Z.; Yang, J.; Chen, H.; Han, L. Environmentally Benign Conversion of Waste Polyethylene Terephthalate to Fluorescent Carbon Dots for "on-off-on" Sensing of Ferric and Pyrophosphate Ions. Journal of Colloid and Interface Science 2019, 538, 481-488.

(42) Hu, Y.; Yang, J.; Tian, J.; Jia, L.; Yu, J. S. Green and Size-Controllable Synthesis of Photoluminescent Carbon Nanoparticles from Waste Plastic Bags. RSC Advances 2014, 4 (88), 47169-47176.

(43) Wang, Y.; Hu, A. Carbon Quantum Dots: Synthesis, Properties and Applications. Journal of Materials Chemistry C 2014, 2 (34), 6921-6939.

(44) Wang, X.; Feng, Y.; Dong, P.; Huang, J. A Mini Review on Carbon Quantum Dots: Preparation, Properties, and Electrocatalytic Application. Frontiers in Chemistry 2019, 7, 671.

(45) Huang, C.; Dong, H.; Su, Y.; Wu, Y.; Narron, R.; Yong, Q. Synthesis of Carbon Quantum Dot Nanoparticles Derived from Byproducts in Bio-Refinery Process for Cell Imaging and in Vivo Bioimaging. Nanomaterials 2019, 9 (3), 387.

(46) Stöber, W.; Fink, A.; Bohn, E. Controlled Growth of Monodisperse Silica Spheres in the Micron Size Range. Journal of Colloid And Interface Science 1968, 26 (1), $62-69$.

(47) Beganskiene, A.; Sirutkaitis, V.; Kurtinaitiene, M.; Juskenas, R.; Kareiva, A. FTIR, TEM and NMR Investigations of Stöber Silica Nanoparticles. Materials Science 2004, 10 (4), 287-290.

(48) Dager, A.; Uchida, T.; Maekawa, T.; Tachibana, M. Synthesis and Characterization of Mono-Disperse Carbon Quantum Dots from Fennel Seeds: Photoluminescence Analysis Using Machine Learning. Scientific Reports 2019, 9 
(1), 1-12.

(49) Yang, H.; Liu, Y.; Guo, Z.; Lei, B.; Zhuang, J.; Zhang, X.; Liu, Z.; Hu, C. Hydrophobic Carbon Dots with Blue Dispersed Emission and Red AggregationInduced Emission. Nature Communications 2019, 10 (1), 1-11.

(50) Lu, S.; Xiao, G.; Sui, L.; Feng, T.; Yong, X.; Zhu, S.; Li, B.; Liu, Z.; Zou, B.; Jin, M.; Tse, J. S.; Yan, H.; Yang, B. Piezochromic Carbon Dots with Two-Photon Fluorescence. Angewandte Chemie 2017, 129 (22), 6283-6287.

(51) Wang, T. Y.; Chen, C. Y.; Wang, C. M.; Tan, Y. Z.; Liao, W. S. Multicolor Functional Carbon Dots via One-Step Refluxing Synthesis. ACS Sensors 2017, 2 (3), 354-363.

(52) Hamid, S. H. Handbook of Polymer Degradation; CRC Press, 2000.

(53) Gardette, M.; Perthue, A.; Gardette, J. L.; Janecska, T.; Földes, E.; Pukánszky, B.; Therias, S. Photo- and Thermal-Oxidation of Polyethylene: Comparison of Mechanisms and Influence of Unsaturation Content. Polymer Degradation and Stability 2013, 98 (11), 2383-2390.

(54) Vogel, C.; Morita, S.; Sato, H.; Noda, I.; Ozaki, Y.; Siesler, H. W. Thermal Degradation of Poly(3-Hydroxybutyrate) and Poly(3-Hydroxybutyrate- Co-3Hydroxyhexanoate) in Nitrogen and Oxygen Studied by ThermogravimetricFourier Transform Infrared Spectroscopy. Applied Spectroscopy 2007, 61 (7), 755-764.

(55) Sun, X.; Lei, Y. Fluorescent Carbon Dots and Their Sensing Applications. $\operatorname{Tr} A C$ - Trends in Analytical Chemistry 2017, 89, 163-180.

(56) Ahn, J.; Song, Y.; Kwon, J. E.; Lee, S. H.; Park, K. S.; Kim, S.; Woo, J.; Kim, H. Food Waste-Driven N-Doped Carbon Dots: Applications for Fe3+ Sensing and Cell Imaging. Materials Science and Engineering C 2019, 102, 106-112.

(57) Dhenadhayalan, N.; Lin, K. C.; Suresh, R.; Ramamurthy, P. Unravelling the Multiple Emissive States in Citric-Acid-Derived Carbon Dots. Journal of Physical Chemistry C 2016, 120 (2), 1252-1261.

(58) Arumugam, S. S.; Xuing, J.; Viswadevarayalu, A.; Rong, Y.; Sabarinathan, D.; Ali, S.; Agyekum, A. A.; Li, H.; Chen, Q. Facile Preparation of Fluorescent Carbon Quantum Dots from Denatured Sour Milk and Its Multifunctional Applications in the Fluorometric Determination of Gold Ions, in Vitro Bioimaging and Fluorescent Polymer Film. Journal of Photochemistry and Photobiology A: Chemistry 2020, 401, 112788.

(59) Sakata, Y.; Uddin, M. A.; Muto, A. Degradation of Polyethylene and Polypropylene into Fuel Oil by Using Solid Acid and Non-Acid Catalysts. Journal of Analytical and Applied Pyrolysis 1999, 51 (1), 135-155.

(60) Chen, Q.; Schönherr, H.; Vancso, G. J. Block-Copolymer Vesicles as Nanoreactors for Enzymatic Reactions. Small 2009, 5 (12), 1436-1445.

(61) Yang, R.; Liu, Y.; Yu, J.; Wang, K. Thermal Oxidation Products and Kinetics of Polyethylene Composites. Polymer Degradation and Stability 2006, 91 (8), 16511657.

(62) Shibryaeva, L. Thermal Oxidation of Polypropylene and Modified Polypropylene - Structure Effects. Polypropylene 2012, 63-86.

(63) Iring, M.; Földes, E.; Barabás, K.; Kelen, T.; Tüdős, F.; Ódor, L. Thermal Oxidation of Linear Low Density Polyethylene. Polymer Degradation and 
Stability 1986, 14 (4), 319-332.

(64) He, M.; Zhang, J.; Wang, H.; Kong, Y.; Xiao, Y.; Xu, W. Material and Optical Properties of Fluorescent Carbon Quantum Dots Fabricated from Lemon Juice via Hydrothermal Reaction. Nanoscale Research Letters 2018, 13 (1), 175.

(65) Zhu, C.; Yang, S.; Wang, G.; Mo, R.; He, P.; Sun, J.; Di, Z.; Kang, Z.; Yuan, N.; Ding, J.; Ding, G.; Xie, X. A New Mild, Clean and Highly Efficient Method for the Preparation of Graphene Quantum Dots without by-Products. Journal of Materials Chemistry B 2015, 3 (34), 6871-6876.

(66) Zhu, S.; Song, Y.; Zhao, X.; Shao, J.; Zhang, J.; Yang, B. The Photoluminescence Mechanism in Carbon Dots (Graphene Quantum Dots, Carbon Nanodots, and Polymer Dots): Current State and Future Perspective. Nano Research 2015, 8 (2), 355-381.

(67) Cayuela, A.; Soriano, M. L.; Carrillo-Carrión, C.; Valcárcel, M. Semiconductor and Carbon-Based Fluorescent Nanodots: The Need for Consistency. Chemical Communications 2016, 52 (7), 1311-1326.

(68) Li, A.; Tang, S.; Tan, P.; Liu, C.; Liang, B. Measurement and Prediction of Oxygen Solubility in Toluene at Temperatures from $298.45 \mathrm{~K}$ to $393.15 \mathrm{~K}$ and Pressures up to 1.0 MPa. Journal of Chemical and Engineering Data 2007, 52 (6), 2339-2344.

(69) Zhang, R.; Adsetts, J. R.; Nie, Y.; Sun, X.; Ding, Z. Electrochemiluminescence of Nitrogen- and Sulfur-Doped Graphene Quantum Dots. Carbon 2018, 129, 45-53.

(70) Sharma, A.; Gadly, T.; Neogy, S.; Ghosh, S. K.; Kumbhakar, M. Molecular Origin and Self-Assembly of Fluorescent Carbon Nanodots in Polar Solvents. Journal of Physical Chemistry Letters 2017, 8 (5), 1044-1052. 
Chapter

Outlook 


\subsection{Introduction}

As part of the rapid developments in nanotechnology, silicon-containing nanoparticles (Si-containing NPs) have become an exciting class of materials holding great promise to serve as enablers in emerging application areas. ${ }^{1-3}$ In this Thesis, we have presented and discussed our efforts to extend the utilization of Si-containing nanoparticles as active ink components for the printing of flexible electronics; as highly effective nucleation agents in polymer foaming; and as nanometric reactors for carbon quantum dot synthesis at relatively mild reaction conditions. However, to move Sicontaining nanoparticles from laboratory research to real-life applications, work remains to be done. This Chapter will briefly introduce and discuss some directions for future research towards the large-scale implementation of silicon nanoparticle-based (SiNPbased) inks for printable electronics. In addition, we present preliminary results for the utilization of the SiNPs used in this Thesis as a promising dual probe in magnetic resonance and fluorescent biomedical imaging.

\subsection{Long-Term Stability and Printability of SiNP- Based Inks}

In Chapter 4, we demonstrated that grafting polymers from SiNP surfaces effectively enhanced the colloidal stability of SiNP inks. With the core-shell hybrid silicon nanoparticles, we successfully prolonged the shelf life of SiNP-based inks and reduced nozzle clogging during inkjet printing. Finally, we demonstrated that the polymer shell is probably responsible for the poor I-V (current-voltage) characteristics of a printed diode. To circumvent this issue, future research must be directed to identify polymer coatings that can stabilize SiNPs-based inks and enhance their printability without leaving too much carbon residue after processing (i.e. annealing) that can negatively affect the electrical properties of printed devices. 


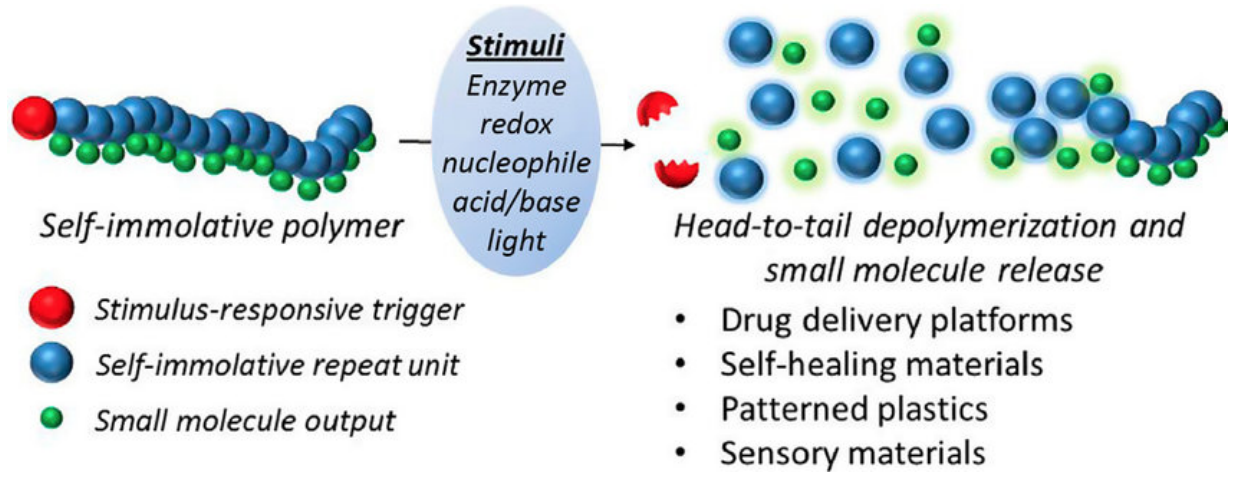

Figure 7.1 Schematic drawing of a self-immolative polymer that undergoes head-to-tail depolymerization upon applying an external stimulus. ${ }^{4}$

Using self-immolating polymers (SIPs) to modify SiNPs could be a promising approach to reduce carbon contamination in printed devices. SIPs can readily depolymerize from end-to-end into small molecules with a relatively low boiling point $\left(\sim 300{ }^{\circ} \mathrm{C}\right)$ upon applying an external stimulus (See Figure 7.1). ${ }^{5}$ Therefore, we believe that the grafted SIPs can be depolymerized after printing by applying a stimulus and the residue can subsequently be vaporized during the sintering process, which effectively reduces carbon contamination in the printed devices while still possessing a prolonged shelf life and enhanced printability for the SiNP-based electronic inks. There are multiple potentially interesting polymers for this purpose, e.g., poly(benzyl carbamates $)^{5}$, poly(carbamates) ${ }^{6}$, poly(benzyl ether) $s^{7}$, poly(phthalaldehyde $)^{8}$ and poly $(\alpha$-methyl styrene $)^{9}$. For example, Hernandez et al. recently reported the fabrication of transient electronics using a metastable 2-(4-methoxystyryl)-4,6-bis(trichloromethyl)-1,3,5triazine/poly(phthalaldehyde) (MBTT/cPPA) substrate. ${ }^{8}$ When triggered by UVirradiation, the MBTT releases $\mathrm{HCl}$, which depolymerizes the cPPA and destroys electronic devices on the substrate without the need of solvents. When the polymer chains are removed, their surface-immobilized anchoring layers can potentially affect printed devices' performance. Therefore the next section provides a suggestion to consider as alternative polymer shell coupling strategy. 


\subsection{Strategies for Polymer Immobilization on SiNP Surfaces}

In Chapter 4, we have demonstrated the use of surface-initiated atom transfer radical polymerization (SI-ATRP) to synthesize core-shell silicon nanoparticles with a shell of poly(methyl methacrylate) (PMMA) and poly(ethylene glycol) methacrylate (PEGMA) brushes. However, the complex reaction steps and harsh reaction conditions limit the capability of these materials for large-scale production. Moreover, the chemicals (e.g., (3-aminopropyl)-triethoxysilane (APTES) and $\alpha$-bromoisobutyryl bromide (BiBB)) used to attach the surface-active ATRP initiators increase the risk of contaminating the printed electrical devices and potentially negatively affect their performance. Therefore, it is essential to select new surface derivation strategies to prepare polymer SiNP hybrids.

a

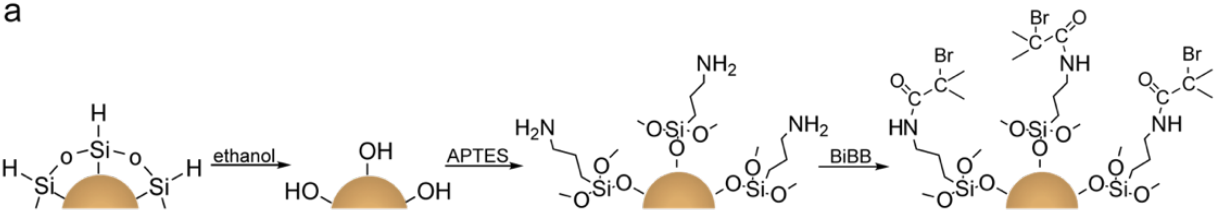

b

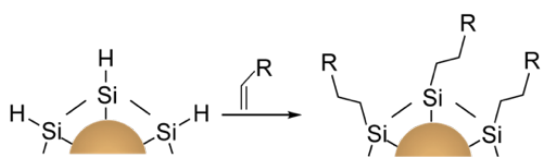

Figure 7.2 Schematic drawing of SiNP surface derivatization strategies to introduce a) ATRP initiator moieties for subsequent SI-ATRP and b) functional groups (R) by hydrosilylation. The $R$ groups are used to attach polymer chains.

Hydrosilylation is a common approach used in organosilicon synthesis. ${ }^{10}$ The recent developments in catalyst-free hydrosilylation make this technique more accessible for the large-scale modification of silicon nanoparticles. ${ }^{11-13}$ Compared to SI-ATRP, the hydrosilylation approach normally introduces less carbon content to the particle surface (see Figure 7.2). Therefore, this method is considered of interest to modify SiNP surfaces before anchoring polymers. However, at the moment, the reaction efficiency for catalystfree hydrosilylation is relatively low compared to Pt-catalyzed hydrosilylation. Increasing the reaction rate is an essential step to enable large-scale utilization of catalystfree hydrosilylation for SiNP modification. 


\subsection{SiNPs as Dual Probe for Magnetic Resonance and Fluorescent Imaging}

Besides the application areas mentioned in this Thesis, Si-containing NPs have also been thoroughly studied in biomedicine (see Chapter 2). Their outstanding properties made SiNPs a promising candidate as magnetic resonance imaging (MRI) contrast agents. Through dynamic nuclear polarization (DNP), a process that temporarily creates a non-equilibrium nuclear spin to enhance MR signal by several orders of magnitudes, ${ }^{14}$ hyperpolarized ${ }^{29} \mathrm{Si}$ MRI allows obtaining positive-contrast background-free imaging since the human body only contains a small amount of silicon. SiNPs also possess a relatively long spin-lattice relaxation time $\left(\mathrm{T}_{1}\right.$, range of few minutes to hours at room temperature). ${ }^{15-17}$

In addition to their promising MRI contrast characteristics, SiNPs are also well known for their photoluminescence (PL). Compared to other toxic heavy metal nanoparticles like CdS and CdSe, SiNPs are ideal for in-vivo fluorescence imaging. ${ }^{18}$ Ruckenstein and coworkers ${ }^{19}$ were one of the first that reported biological imaging with SiNPs. They demonstrated in-vitro imaging of Chinese hamster ovary cells using polyacrylic acid-terminated SiNPs. To date, numerous efforts have been reported that utilize SiNPs for in-vitro and in-vivo fluorescence imaging. ${ }^{20-22}$

Inspired by the exciting SiNPs material characteristics mentioned above, we made some first attempts to utilize the obtained SiNPs (boron doped, $10^{18}$ atoms $\mathrm{cm}^{-3}$ ) as a dual probe for hyperpolarized ${ }^{29} \mathrm{Si} \mathrm{MRI}$ and fluorescent imaging.

Excitation Dependent Photoluminescence. The SiNPs used in Chapter 3 and Chapter 4 were suspended in ethylene glycol at a concentration of $120 \mathrm{ppm}$, and then emission spectra of the suspension were measured with a photoluminescent spectrometer. Figure 7.3 presents the emission spectrum of the SiNPs for excitation wavelengths between $440 \mathrm{~nm}$ to $540 \mathrm{~nm}$. All spectra were recorded under identical conditions. Thus, the measured intensities are directly comparable. From Figure 7.3, it is clear that the SiNPs show a strong excitation-wavelength-dependent fluorescence emission. Upon increasing the excitation wavelength $\left(\lambda_{\mathrm{ex}}\right)$ from 440 to $540 \mathrm{~nm}$, the emission peaks gradually shift from 590 to $700 \mathrm{~nm}$. The highest emission intensity is observed at $\lambda_{\mathrm{em}}=$ $620 \mathrm{~nm}$ with $\lambda_{\mathrm{ex}}=460 \mathrm{~nm}$. The intensity of the emission peaks decreases upon increasing $\lambda_{\text {ex }}$ from $460 \mathrm{~nm}$ to $540 \mathrm{~nm}$. 


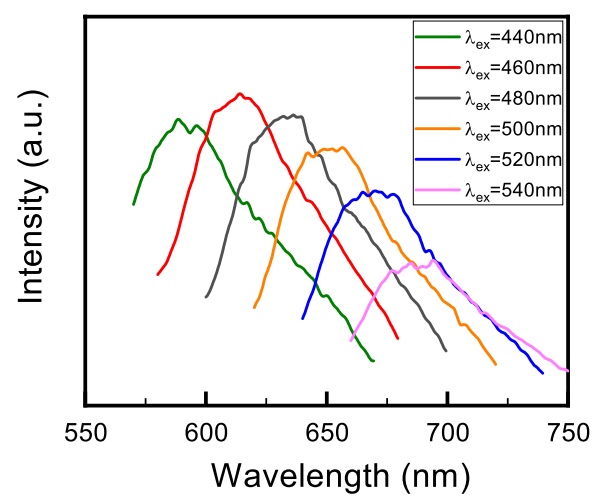

Figure 7.3 Excitation-dependent PL emission of SiNPs. The excitation wavelength increased from $440 \mathrm{~nm}$ to $540 \mathrm{~nm}$.

The exact mechanism for silicon nanoparticle fluorescence is still under debate. However, it is widely agreed that the fluorescence emission is originated from the recombination of excited electron-hole pairs in the quantum-dimensional nanocrystalline core. Those nanocrystalline cores behave as direct bandgap materials during excitation. The excitation-dependent fluorescence is likely due to the size dispersity of the crystal domains embedded in the primary particles. $^{23}$ Since the bandgap of the silicon nanoparticles is directly related to the size of the nanocrystalline cores, only the crystalline core with the corresponding diameter (bandgap) will contribute to the luminescence emission. ${ }^{23,24}$ The maximum emission intensity $\left(\lambda_{\mathrm{em}}=620 \mathrm{~nm}\right)$ corresponds to a nanocrystalline core with a diameter of $3.74 \mathrm{~nm} .^{23}$

${ }^{29}$ Si Magnetic Resonance Imaging. Long $\mathrm{T}_{1}$ time is critical for targetable DNP MRI agents. In this part, we focused on measuring the $\mathrm{T}_{1}$ time of our SiNPs using DNP. Prior to DNP, the electron paramagnetic resonance (EPR) of the SiNPs was measured to confirm the presence of free electrons that can be directly hyperpolarized. The EPR spectrum at $4.2 \mathrm{~K}$ (Figure 7.4a) shows one resonance peak with a g-value of 2.0055, corresponding to paramagnetic point defects at the interface of crystalline $\mathrm{Si}$ and $\mathrm{SiO}_{2}{ }^{25}$ Therefore, the free electrons in the SiNPs are primarily located in the surface defects between the crystalline $\mathrm{Si}$ and $\mathrm{SiO}_{2}$ phase. The DNP profiles from frequency modulated (FM) microwave irradiation and continuous wave (CW) irradiation at $4.2 \mathrm{~K}$ are shown in Figure 7.4b. It can be observed that the FM irradiation shows considerably higher DNP enhancement compared to $\mathrm{CW}$ irradiation. The observed intensity difference between FM and CW irradiation for SiNPs is because FM irradiation can prevent dephasing and relaxation effects during hyperpolarization. ${ }^{26}$ The DNP build-up curve of SiNPs is shown in Figure 7.4c. DNP build-up curves were recorded with a series of pulse-acquire 178 
experiments with a repetition time of 60 s and a low $\sim 15$ degree flip angle. The DNP build-up time for SiNP is $937 \pm 33 \mathrm{~s}$, which is considerably shorter than the value reported for SiNPs in the literature. ${ }^{27}$ The magnetization decay of polarized SiNPs was measured at $4 \mathrm{~K}$ (Figure $7.4 \mathrm{~d}$ ). The $\mathrm{T}_{1}$ extrapolated from the magnetization decay for SiNPs at $4 \mathrm{~K}$ is approximately $570 \mathrm{~s}$. As a next step, we note that the SiNPs' T1 at physiological conditions must be determined before we can conclude that these particles are useful as a probe in dual imaging.
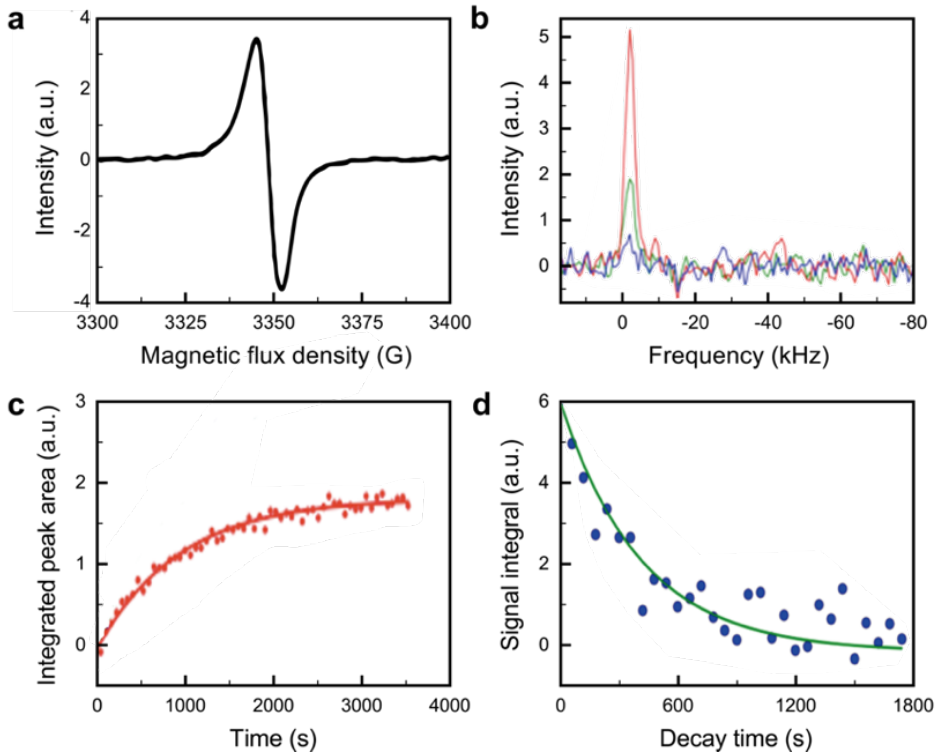

Figure 7.4 a) EPR spectrum of SiNPs. The g-value is 2.0055. b) DNP profiles of SiNPs with (FM microwave irradiation (red) and CW irradiation (green) at $4.2 \mathrm{~K}$. c) DNP buildup curves of SiNPs at $4.2 \mathrm{~K}$ using FM irradiation. d) The magnetization decay of the SiNPs at $4.2 \mathrm{~K}$ after 4 hours of hyperpolarization.

Overall, these preliminary results show that the SiNPs are magnetic and visually responsive, rendering them a promising candidate to serve as a dual probe for hyperpolarized ${ }^{29}$ Si MRI and fluorescent biomedical imaging. Future work should focus on i) SiNPs surface modification with organic molecules/polymers to improve the solubility and stability in biofluids, ii) extrapolate the relation between size, $T_{1}$, and an optimal MRI signal at room temperature, and iii) perform laboratory as well as in-vivo tests to evaluate the combined hyperpolarized ${ }^{29} \mathrm{Si} \mathrm{MRI}$ and fluorescent imaging capabilities. 


\subsection{References}

(1) Chang, H.; Sun, S. Q. Silicon Nanoparticles: Preparation, Properties, and Applications. Chinese Physics B 2014, 23 (8), 1-14.

(2) Jeelani, P. G.; Mulay, P.; Venkat, R.; Ramalingam, C. Multifaceted Application of Silica Nanoparticles. A Review. Silicon 2020, 12 (6), 1337-1354.

(3) Nayfeh, M. H.; Mitas, L. (2008) Silicon Nanoparticles. New Photonic and Electronic Material at the Transition Between Solid and Molecule. In V. Kumar (Ed.). Nanosilicon (pp 1-78). Elsevier.

(4) Peterson, G. I.; Larsen, M. B.; Boydston, A. J. Controlled Depolymerization: Stimuli-Responsive Self-Immolative Polymers. Macromolecules 2012, 45 (18), 7317-7328.

(5) Sagi, A.; Weinstain, R.; Karton, N.; Shabat, D. Self-Immolative Polymers. Journal of the American Chemical Society 2008, 130 (16), 5434-5435.

(6) Robbins, J. S.; Schmid, K. M.; Phillips, S. T. Effects of Electronics, Aromaticity, and Solvent Polarity on the Rate of Azaquinone-Methide-Mediated Depolymerization of Aromatic Carbamate Oligomers. Journal of Organic Chemistry 2013, 78 (7), 3159-3169.

(7) Olah, M. G.; Robbins, J. S.; Baker, M. S.; Phillips, S. T. End-Capped Poly(Benzyl Ethers): Acid and Base Stable Polymers That Depolymerize Rapidly from Headto-Tail in Response to Specific Applied Signals. Macromolecules 2013, 46 (15), 5924-5928.

(8) Lopez Hernandez, H.; Kang, S. K.; Lee, O. P.; Hwang, S. W.; Kaitz, J. A.; Inci, B.; Park, C. W.; Chung, S.; Sottos, N. R.; Moore, J. S.; Rogers, J. A.; White, S. R. Triggered Transience of Metastable Poly(Phthalaldehyde) for Transient Electronics. Advanced Materials 2014, 26 (45), 7637-7642.

(9) Malhotra, S. L.; Baillet, C.; Minh, L.; Blanchard, L. P. Thermal Decomposition of Poly- $\alpha$-Methylstyrene. Journal of Macromolecular Science: Part A - Chemistry 1978, 12 (1), 129-147.

(10) Hofmann, R. J.; Vlatkovic, M.; Wiesbrock, F. Fifty Years of Hydrosilylation in Polymer Science: A Review of Current Trends of Low-Cost Transition-Metal and Metal-Free Catalysts, Non-Thermally Triggered Hydrosilylation Reactions, and Industrial Applications. Polymers 2017, 9 (10), 534.

(11) Lie, L. H.; Duerdin, M.; Tuite, E. M.; Houlton, A.; Horrocks, B. R. Preparation and Characterisation of Luminescent Alkylated-Silicon Quantum Dots. Journal of Electroanalytical Chemistry 2002, 538, 183-190.

(12) Rogozhina, E. v.; Eckhoff, D. A.; Gratton, E.; Braun, P. v. Carboxyl Functionalization of Ultrasmall Luminescent Silicon Nanoparticles through Thermal Hydrosilylation. Journal of Materials Chemistry 2006, 16 (15), 14211430.

(13) Yu, Y.; Hessel, C. M.; Bogart, T. D.; Panthani, M. G.; Rasch, M. R.; Korgel, B. A. Room Temperature Hydrosilylation of Silicon Nanocrystals with Bifunctional Terminal Alkenes. Langmuir 2013, 29 (5), 1533-1540.

(14) Seo, H.; Choi, I.; Whiting, N.; Hu, J.; Luu, Q. S.; Pudakalakatti, S.; McCowan, C.; Kim, Y.; Zacharias, N.; Lee, S.; Bhattacharya, P.; Lee, Y. Hyperpolarized Porous Silicon Nanoparticles: Potential Theragnostic Material for ${ }^{29} \mathrm{Si}$ Magnetic 
Resonance Imaging. ChemPhysChem 2018, 19 (17), 2143-2147.

(15) Atkins, T. M.; Cassidy, M. C.; Lee, M.; Ganguly, S.; Marcus, C. M.; Kauzlarich, S. M. Synthesis of Long $\mathrm{T}_{1}$ Silicon Nanoparticles for Hyperpolarized ${ }^{29} \mathrm{Si}$ Magnetic Resonance Imaging. ACS Nano 2013, 7 (2), 1609-1617.

(16) Lilburn, D. M. L.; Pavlovskaya, G. E.; Meersmann, T. Perspectives of Hyperpolarized Noble Gas MRI beyond 3He. Journal of Magnetic Resonance 2013, 229, 173-186.

(17) Keshari, K. R.; Wilson, D. M. Chemistry and Biochemistry of ${ }^{13} \mathrm{C}$ Hyperpolarized Magnetic Resonance Using Dynamic Nuclear Polarization. Chem Soc Rev. 2014, 43 (5), 1627-1659.

(18) Peng, F.; Su, Y.; Zhong, Y.; Fan, C.; Lee, S. T.; He, Y. Silicon Nanomaterials Platform for Bioimaging, Biosensing, and Cancer Therapy. Accounts of Chemical Research 2014, 47 (2), 612-623.

(19) Li, Z. F.; Ruckenstein, E. Water-Soluble Poly(Acrylic Acid) Grafted Luminescent Silicon Nanoparticles and Their Use as Fluorescent Biological Staining Labels. Nano Letters 2004, 4 (8), 1463-1467.

(20) Shiohara, A.; Hanada, S.; Prabakar, S.; Fujioka, K.; Lim, T. H.; Yamamoto, K.; Northcote, P. T.; Tilley, R. D. Chemical Reactions on Surface Molecules Attached to Silicon Quantum Dots. Journal of the American Chemical Society 2010, 132 (1), 248-253.

(21) Zhou, Y.; Zhang, Y.; Zhong, Y.; Fu, R.; Wu, S.; Wang, Q.; Wang, H.; Su, Y.; Zhang, H.; He, Y. The in Vivo Targeted Molecular Imaging of Fluorescent Silicon Nanoparticles in Caenorhabditis Elegans. Nano Research 2018, 11 (5), 23362346.

(22) Cao, Z.; Peng, F.; Hu, Z.; Chu, B.; Zhong, Y.; Su, Y.; He, S.; He, Y. In Vitro Cellular Behaviors and Toxicity Assays of Small-Sized Fluorescent Silicon Nanoparticles. Nanoscale 2017, 9 (22), 7602-7611.

(23) Kim, T. Y.; Park, N. M.; Kim, K. H.; Sung, G. Y.; Ok, Y. W.; Seong, T. Y.; Choi, C. J. Quantum Confinement Effect of Silicon Nanocrystals in Situ Grown in Silicon Nitride Films. Applied Physics Letters 2004, 85 (22), 5355-5357.

(24) Meier, C.; Gondorf, A.; Lüttjohann, S.; Lorke, A.; Wiggers, H. Silicon Nanoparticles: Absorption, Emission, and the Nature of the Electronic Bandgap. Journal of Applied Physics 2007, 101 (10), 103112.

(25) Stesmans, A.; Jivanescu, M.; Godefroo, S.; Zacharias, M. Paramagnetic Point Defects at $\mathrm{SiO}_{2} /$ Nanocrystalline Si Interfaces. Applied Physics Letters 2008, 93 (2), 023123.

(26) Blazina, D.; Duckett, S. B.; Halstead, T. K.; Kozak, C. M.; Taylor, R. J. K.; Anwar, M. S.; Jones, J. A.; Carteret, H. A. Generation and Interrogation of a Pure Nuclear Spin State by Parahydrogen-Enhanced NMR Spectroscopy: A Defined Initial State for Quantum Computation. Magnetic Resonance in Chemistry 2005, 43 (3), 200-208.

(27) Kwiatkowski, G.; Jähnig, F.; Steinhauser, J.; Wespi, P.; Ernst, M.; Kozerke, S. Nanometer Size Silicon Particles for Hyperpolarized MRI. Scientific Reports 2017, 7 (1), 7946. 



\section{Summary}

Due to the swift progress in nanotechnology new and exciting opportunities have become within reach for nanoparticles. Great progress has been made in utilizing siliconcontaining nanoparticles in various applications, e.g., in drug delivery, bio-sensing, energy (solar cell) utilization, and energy storage. The work presented in this Thesis aims at exploiting the frontier of silicon-containing nanoparticles from the application perspective. Both silicon ( $\mathrm{Si} \mathrm{NPs})$ and silica $\left(\mathrm{SiO}_{2} \mathrm{NPs}\right)$ nanoparticles have formed target systems.

In Chapter 2, a literature review, introducing the general aspects and synthetic strategies of silicon-based nanoparticles are provided. In addition, a detailed overview of the utilization of silicon-based nanoparticles in emerging application areas, including printing of electronic devices, biomedical imaging, nanocellular foaming, and catalysis, are also reviewed. The limitations and challenges hampering the successful integration of silicon-based nanoparticles into real-life applications are presented and discussed, as well.

As the first step of formulating Si NPs-based printable electronic inks, in Chapter 3, the long-term colloidal stability of SiNPs with complex geometry and broad size distribution are studied in detail,combining theoretical and experimental approaches. The colloidal stability of SiNPs in different alcohols and glycols is evaluated by applying Stokes' law. In addition, the long-term colloidal stability of SiNP dispersions is investigated with experimental approaches, i.e. analytical centrifugation and batch sedimentation. Furthermore, the limitations of using theories and analytical centrifugation to predict the long-term colloidal stability of SiNPs with complex geometries and broad size distributions are elucidated. Eventually, based on the results presented and discussed in this Chapter, we conclude that surface modification of the SiNPs is needed to obtain SiNP inks with the required dispersion stability.

In Chapter 4, core-shell SiNP/polymer hybrids are prepared to enhance the printability of SiNP-based inks introduced in Chapter 3. The SiNP/polymer hybrid-based inks are tested with inkjet printing and spin coating. Moreover, the influence of the grafted polymer on the electrical properties of the printed electronic devices' is also thoroughly investigated. Using core-shell SiNP/polymer hybrids is shown to constitute an effective approach to reduce nozzle clogging during inkjet printing. Furthermore, the 
polymer shell prevents undesirable chemical reactions between particle and solvent from occurring, which broadens the range of solvents suitable for ink formulation. However, the grafted polymer shell around the SiNPs has a negative impact on the performance of the printed electronic devices fabricated with these NPs. This points out the urgency to search for alternative polymers and immobilization strategies to reduce the influence of polymer grafts on the functionality of printed devices, while maintaining enhanced colloidal stability and improved printability of the SiNP-based inks.

Besides $\mathrm{SiNPs}, \mathrm{SiO}_{2}$ are a fascinating class of silicon-containing nanoparticles, and they are investigated in two applications at the frontiers of nanoparticle science in this Thesis, as well. Surface roughness engineered $\mathrm{SiO}_{2} \quad\left(\mathrm{SiO}_{2}-\mathrm{R}\right) \quad \mathrm{NPs}$ with poly(dimethylsiloxane) (PDMS) shells are presented in Chapter $\mathbf{5}$ as nucleating agents in the $\mathrm{CO}_{2}$ batch foaming of poly(methyl methacrylate) (PMMA) nanocellular foams. We showed that the nanocavities (concave surfaces) on $\mathrm{SiO}_{2}-\mathrm{R}$ NPs can act as heterogeneous nucleation centers. We concluded that the concave surface effectively reduces the free energy for foam cell nucleation. The PDMS decorated $\mathrm{SiO}_{2}-\mathrm{R} \mathrm{NPs}$ can nucleate multiple foam cells from a single particle. Due to capillary condensation inside the nanocavities, $\mathrm{SiO}_{2}-\mathrm{R}$ NPs can absorb more $\mathrm{CO}_{2}$ during saturation than their nearly smooth counterparts. Moreover, the PDMS shell boosts the $\mathrm{CO}_{2}$ uptake, resulting in multiple nucleation sites on a single particle. This results in a nucleation efficiency of 6.2 for $200 \mathrm{~nm}$ PDMS coated $\mathrm{SiO}_{2}$-Rs, which is 40 times higher than their nearly smooth counterparts.

In Chapter 6, $\mathrm{SiO}_{2} \mathrm{NP}$ aggregates are used as enablers to fabricate carbon quantum dot (CQD) fluorescent markers containing polyethylene (PE). The $\mathrm{SiO}_{2} \mathrm{NP}$ aggregates are obtained by suspending monodispersed $\mathrm{SiO}_{2} \mathrm{NPs}$ in toluene. The pores on the aggregates act as nanometric reactors and facilitate CQD formation when PE chains enter while the solution is heated. Strong blue fluorescence is observed from the resulting PE/CQD composites. Upon decreasing the reaction temperature from $110{ }^{\circ} \mathrm{C}$ to $90{ }^{\circ} \mathrm{C}$, the maximum emission wavelength shifts from $394 \mathrm{~nm}$ to $408 \mathrm{~nm}$ for PE/CQD composites. This shift in emission wavelength is attributed to the increased oxygen content in the reaction mixture upon decreasing the reaction temperature. The CQDs can be extracted from PE/CQD composites via a simple freeze-melt process. The extracted CQDs have an average diameter of $2.3 \pm 0.5 \mathrm{~nm}$, as is obtained by TEM image analysis. Finally, we demonstrate that PE/CQD composites and extracted CQDs marked PE samples could be visually separated from pure unmarked PE upon irradiation with 367 nm light. 
In Chapter 7, we suggest future research directions for SiNP-based inks. We exploit the possible use of self-immolating polymers and catalyst-free hydrosilylation as the next essential step to reduce the impact of polymer coating on the performance of printed electronic devices. Moreover, we present preliminary results on utilizing multicrystalline SiNPs as a dual imaging agent for magnetic resonance imaging and fluorescent imaging. 



\section{Samenvatting}

Vanwege de snelle vooruitgang in de nanotechnologie zijn er allerlei nieuwe en interessante mogelijkheden binnen handbereik gekomen voor nanodeeltjes. Er is veel vooruitgang geboekt om silicium-houdende nanodeeltjes in verscheidene toepassingen, zoals medicijnen, biosensoren, energie opwekking (zonnecellen), en energie opslag, te gebruiken. Het in dit proefschrift uiteengezette werk richt zich er op om vanuit een toepassingsperspectief het uiterste uit silicium-houdende nanodeeltjes te halen. Zowel silicium ( $\mathrm{SiNPs})$ als silica $\left(\mathrm{SiO}_{2} \mathrm{NPs}\right)$ nanodeeltjes zijn hiervoor gebruikt.

In Hoofdstuk 2, een literatuur overzicht, worden algemene aspecten en synthese strategieën voor op silicium gebaseerde nanodeeltjes gegeven. Hiernaast wordt een gedetailleerd overzicht voor het gebruik van op silicium gebaseerde nanodeeltjes, waaronder het printen van elektronica, biomedisch imaging, nanocellulair schuimen, en katalyse, gegeven. De beperkingen alsook de uitdagingen die de succesvolle toepassing van op silicium gebaseerde nanodeeltjes in de weg staan, worden ook uiteengezet en besproken.

In Hoofdstuk 3 wordt als eerste stap voor de op SiNPs gebaseerde printbare, elektronische inkt-formulering, de colloïdale stabiliteit van SiNPs met complexe geometrie en brede distributie van de deeltjesgrootte, in detail bestudeerd, waarbij theoretische en experimentele benaderingen worden gecombineerd. De colloïdale stabiliteit van SiNPs in verschillende alcoholen en glycolen wordt hier geëvalueerd door middel van de Wet van Stokes. Daarnaast wordt de colloïdale stabiliteit van SiNP suspensies op de lange termijn onderzocht met behulp van experimentele benaderingen, d.w.z., analytisch centrifugeren en batch sedimentatie. Ook worden de beperkingen van het gebruik van theorieën alsook analytisch centrifugeren om de colloïdale stabiliteit van SiNPs met complexe geometrie en brede distributie van de deeltjesgrootte op de lange termijn te bepalen, verder uitgediept. Uiteindelijk, gebaseerd op de resultaten die gepresenteerd en uitgelegd zijn in dit Hoofdstuk, concluderen we dat oppervlakte aanpassingen van SiNPs nodig is om een SiNP inkt te kunnen maken met de vereiste dispersie stabiliteit.

In Hoofdstuk 4 worden core-shell SiNP/polymer hybride deeltjes gemaakt om de printbaarheid van op SiNP gebaseerde inkt, zoals geïntroduceerd in Hoofdstuk 3. De $\mathrm{SiNP} /$ polymer hybride inkten zijn getest door middel van inkjet printen en spin coating. 
Hiernaast wordt ook de invloed van de geënte polymeer op de elektrische eigenschappen van de geprinte elektronische apparaten uitvoerig bestudeerd. Van het gebruik van coreshell SiNP/polymer hybride deeltjes wordt aangetoond dat het een effectieve manier is om verstopping van printmkoppen tijdens het printen te voorkomen. Verder voorkomt de polymeer schil dat ongewenste chemische reacties tussen de deeltjes en de oplosmiddelen plaatsvindt. Echter, de geënte polymeer schil rondom de SiNPs heeft een negatieve impact op de prestatie van de met deze nanodeeltjes gemaakte, geprinte elektronische apparaten. Dit benadrukt de noodzaak om in volgende stappen te zoeken naar alternatieve polymeren en immobilisatie strategieën om de invloed van geënte polymeren op de functionaliteit van geprinte apparaten met behoud van de verbeterde colloïdale stabiliteit en verbeterde printbaarheid van de op SiNPs gebaseerde inkten te verminderen.

Naast $\mathrm{SiNPs}$ zijn ook $\mathrm{SiO}_{2}$ deeltjes een interessante groep van silicium houdende nanodeeltjes. Deze groep wordt ook in dit proefschrift onderzocht door middel van twee toepassingen op de grenzen van het nanodeeltjes-vakgebied. Het gebruik van $\mathrm{SiO}_{2}$ deeltjes met ruwe oppervlaktes $\left(\mathrm{SiO}_{2}-\mathrm{R}\right)$ met polydimethylsiloxaan (PDMS) schillen als cel nucleatiekernen wordt gedemonstreerd in Hoofdstuk 5 door middel van het $\mathrm{CO}_{2}$ batch schuim proces van polymethyl methacrylate (PMMA) nanocellulaire schuimen. We laten zien dat de nanoholtes (met concaaf oppervlak) op de $\mathrm{SiO}_{2}-\mathrm{R}$ NPs kunnen dienen als heterogene nucleatie kernen. We concluderen dat het concave oppervlak de vrije energie, benodigd voor schuim cel nucleatie, verlaagd. De, in PDMS gehulde, $\mathrm{SiO}_{2}$ $\mathrm{R}$ NPs kunnen meer $\mathrm{CO}_{2}$ absorberen tijdens de verzadigingsstap dan de gladde tegenhangers. Ook verbeteren de PDMS schillen de $\mathrm{CO}_{2}$ opname waardoor meerdere nucleaties kunnen plaatsvinden op één enkel deeltje. Dit leidt tot een nucleatie efficiëntie van 6.2 voor met PDMS gehulde $\mathrm{SiO}_{2}-\mathrm{R}$ NPs, wat maar liefst 40 keer hoger is dan voor de gladde tegenhangers.

In Hoofdstuk 6 worden $\mathrm{SiO}_{2} \mathrm{NP}$ aggregaten gebruikt als platform om fluorescente,

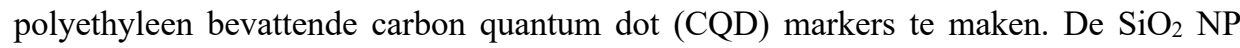
aggregaten zijn verkregen door middel van de suspensie van monodisperse $\mathrm{SiO}_{2} \mathrm{NPs}$ in tolueen. De poriën op de aggregaten dienen als nanoreactoren en faciliteren CQD formatie wanneer de polyethyleen ketens zich hierin bevinden terwijl de oplossing wordt verhit. Intense blauwe fluorescentie wordt geobserveerd in de PE/CQD composieten. Wanneer de reactie temperatuur van $110{ }^{\circ} \mathrm{C}$ naar $90{ }^{\circ} \mathrm{C}$ verlaagd wordt, verschuift de emissie golflengte van $394 \mathrm{~nm}$ naar $408 \mathrm{~nm}$ voor de PE/CQD composieten. Deze verschuiving in emissie golflengte wordt toegedicht aan het verhoogde zuurstof gehalte in het reactie mengsel wanneer de temperatuur verlaagd wordt. De CQDs kunnen uit het $\mathrm{PE} / \mathrm{CQD}$ composiet gehaald worden door middel van een simpel vreis-smelt proces. De 
geëxtraheerde CQDs hebben een gemiddelde diameter van $2.3 \pm 0.5 \mathrm{~nm}$, zoals gemeten met TEM analyse. Concluderend, we hebben van PE/CQD composieten en met geëxtraheerde CQDs gemarkeerde PE monsters laten zien dat ze op visuele basis onderscheiden kunnen worden van schone, ongemarkeerde PE onder bestraling met licht van $367 \mathrm{~nm}$ golflengte.

In Hoofdstuk 7 stellen we voor op SiNPs gebaseerde inkt, een aantal potentiële onderzoeksrichtingen voor. We verkennen het potentieel gebruik van zelf-verbrandende polymeren en katalysatorvrije hydrosilylatie als de volgende essentiële stap om de invloed van polymeer coatings op de prestatie van geprinte elektronische apparaten. Hiernaast presenteren we ook de voorlopige resultaten voor het gebruik van multikristallijne SiNPs als een tweezijdig imaging agent voor magnetic resonance imaging (MRI) en fluorescentie imaging. 



\section{Acknowledgements}

Before finishing the Thesis, I want to dedicate this section to express my deepest gratitude to all those who have supported me along these years.

First and foremost, I would love to express my deepest gratitude and sincere appreciation to my promoter, Prof. Julius Vancso. Dear Julius, it is a great pleasure and honor to be your student and to be part of the SPC (MTP). You are a serious, rigorous, and enthusiastic scientist in teaching and scientific research. Your broad knowledge and precise perspectives towards science and technology have benefited me a lot. Your patient guidance and encouragement provided me the great motivation and enjoyments that kept me going.

My gratitude and appreciation also transfer to my supervisor/assistant promotor, Dr. Joost Duvigneau. Dear Joost, thank you for your supervision and help during all these years. Your professional and sharp insights towards scientific research and writing have inspired me a lot. Every discussion and meeting with you end with constructive suggestions and encouragements, which highly motivated me in fulfilling my research activities. I am very grateful for the plentiful efforts and time that you have spent on guiding me. You are not only a great supervisor, but also a great friend for me. I enjoyed every scientific discussion and daily conversation with you.

I would also like to thank my daily supervisor Dr. Olivier Nguon. Dear Olivier, thank you for your guidance in the first two years of my Ph.D. Starting a Ph.D is always hard and sometimes confusing. I am lucky to have you there to guide me through the first two years. Your sharp insights and rigorous attitude towards scientific research are an inspiration to me.

Dearest Julius, Joost, and Olivier, without the guidance and support from you, the work described in this Thesis would not be accomplished. Words are powerless to express my gratitude and thanks to all of you. I sincerely wish you and your families all the best in the coming future.

I would also like to thank all the other collaborators in the Thesis: Prof. Niels Benson, Fabian Langer, Laura Kühnel, Juha Niittynen, Joost Valeton, Dr. Dominique ThiesWeesie, Paul H.H. Bomans. Thanks, Prof. Niels Benson, Fabian Langer, Laura Kühnel for your help and valuable discussion in inkjet printing and diode processing of silicon 
nanoparticle inks. Thanks, Juha Niittynen and Joost Valeton for the help and valuable discussion in inkjet printing of silicon nanoparticles. Thanks, Dr. Dominique ThiesWeesie for the help and valuable discussion of analytical centrifugation of silicon nanoparticle inks. Thanks Paul H.H. Bomans for conducting the high-resolution transmission electron microscopy measurements.

I would also like to thank my defense committee members, Prof. Leon Lefferts, Prof. Anke Blume, Prof Niels Benson, Prof. Mikael Hedenqvist, Dr. Olivier Nguon, for your time to read my thesis and give me constructive suggestions.

The next person I would like to express my sincere gratitude to is Clemens Padberg. Dear Clemens, you are one of the most amazing persons in the SPC (MTP) group. You can fix almost every instrument failure in our group (and others) and are always willing to help with anything. This makes everyone chase after you for help every day, certainly, including me. But no matter how busy you are, you are always glad and timely to help me.

Grateful thanks to our secretaries, Romon, Marion, Nicole, and Izabel, for your help. You are always there when I need help, whether dealing with various tedious TGS processes or providing support for paperwork.

Next, I would like to give my special thanks to my best Chinese friends from MTP, Kaihuan, Yan, Shanqiu, Yunchong, Huan, and Jinmeng. Thank you for your assistance in my earlier life and research work. I wish you and your loved ones all the best.

I also wish to thank all the present and former SPC (MTP) members. Thanks a lot for the help from you guys, and I really enjoyed the time we have spent together. It is of great pleasure to have the chance to work with you.

Finally, I would like to express my most enormous thanks to my family members who always support me, my parents (殷建立, 刘静), my wife (石湘雯). I am very grateful for the endless love and support from them. Without all your suggestions and support, I would not be at the point where I am now. Sincerely wish all of you a healthy and happy life. I love you all.

殷思达

Sida Yin

Enschede, 2021 


\section{List of Publications}

$>$ Journal articles:

1. Liu, S.; Yin, S.; Duvigneau, J.; Vancso, G. J. Bubble Seeding Nanocavities: Multiple Polymer Foam Cell Nucleation by Polydimethylsiloxane-Grafted Designer Silica Nanoparticles. ACS Nano 2020, 14 (2), 1623-1634.

2. Yin, S.; Duvigneau, J.; Vancso, G. J. Fluorescent polyethylene by in-situ facile synthesis of carbon quantum dots facilitated by silica nanoparticle agglomerates, Accepted

$>$ Manuscripts:

1. Yin, S.; Nguon, O. J.; Duvigneau, J.; Vancso, G. J. Dispersion stability of nonspherical silicon nanoparticles: impact of solvent selection and storage time, submitted.

2. Yin, S.; Duvigneau, J.; Vancso, G. J. Emerging applications of siliconcontaining nanoparticles, in preparation. 



\section{Curriculum Vitae}

Sida Yin was born on Aug. 04 $4^{\text {th }}, 1990$ in Hebei China. After finishing his B.Sc. in 2014, he continued his master studies in chemical engineering at Univiersity of Twente in the same year. This research project was conducted in the group of Prof. dr. Julius Vancso and focused on the $\mathrm{CO}_{2}$ assisted nanocellular polymer foraming with silica nanoparticles as efficient nucleation agents. After graduation in 2016, he continued as a Ph.D. student under the supervision of Prof. dr. Julius Vancso and Dr. Joost Duvigneau in the group of Materials Science and Technology of Polymers (MTP) (later Sustainable Polymer Chemistry group) at the University of Twente. His Ph.D. project focused on utilizing the application potential of Si-containing NPs ( $\mathrm{SiNPs}$ and $\mathrm{SiO}_{2} \mathrm{NPs}$ ) to ensure continuous progress in the field. The result of his research are described in this Thesis. 
Silicon piitä मिक्सीवर्ठ

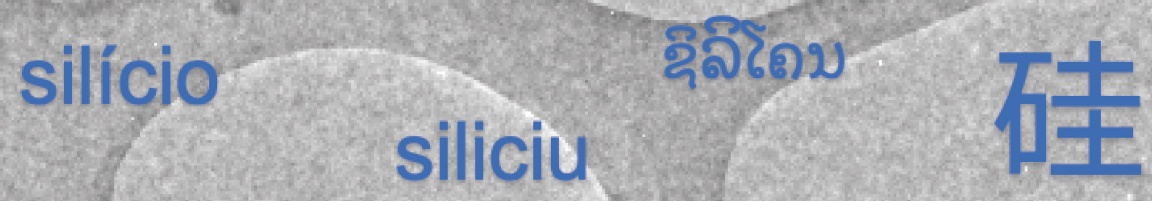

sîlîkon

nesilicon

lesilicon

uhLhghnu /

silīcijs kremniý

silizioa

$\pi, n, h 3$

Pii

pakachitsulo

silicio Silizium

อิติตุุ | silicium

räni

silicio

Oיציציציביטי

\section{sileacain}

సిలికాన్

kisel

silisium

кремний सिलिकन

kremik

тupítı 